\title{
Regiocontrolled Reductive Vinylation of Aliphatic 1,3- Dienes with Vinyl Triflates by Nickel Catalysis
}

Xiaobo Pang, Zhen-Zhen Zhao, Xiao-Xue Wei, Liangliang Qi, Guang-Li Xu, Jicheng Duan, Xue-Yuan Liu, Xing-Zhong Shu*

State Key Laboratory of Applied Organic Chemistry (SKLAOC), College of Chemistry and Chemical Engineering, Lanzhou University, 222 South Tianshui Road, Lanzhou, 730000, China.

\section{Table of Contents}

1. General Information.......................................................

2. Synthesis of Ligands..................................................S3

3. Preparation of Starting Materials....................................S7

4. Regiocontrolled Reductive Vinylation of Aliphatic 1,3-Dienes with Vinyl Triflates by Nickel Catalysis..........................................S18

5. Mechanistic Investigation........................................S44

6 Synthesis and Crystallographic Data of Complex A....................S52

7. References......................................................S76

8. Copies of NMR Spectra..........................................S78

Scheme S1...page S46 Scheme S2...page S49 Scheme S3 and S4...page S51 


\section{General Information}

All reactions were carried out under an atmosphere of argon in sealed tube with magnetic stirring. Dry THF were purified using a solvent-purification system that contained activated alumina and molecular sieves. DIPE (Diisopropyl ether) and other solvents were dried and purified according to the procedure from "Purification of Laboratory Chemicals". ${ }^{1}$

Nickel catalysts were purchased from Acros, Alfa Aesar, Ark Pharm, and Strem. Other chemicals were purchased from TCI, Adamas, Aldrich, J\&K scientific and Energy chemicals, and were directly used without further purifications.

${ }^{1} \mathrm{H}$ and ${ }^{13} \mathrm{C}$ NMR spectra were collected on a Bruker AVANCE III $400 \mathrm{MHz}$, JEOL JNM-ECS 400M and Agilent-NMR-inova $600 \mathrm{MHz}$ spectrometer at room temperature. ${ }^{1} \mathrm{H}$ NMR spectra were reported in parts per million (ppm) downfield of tetramethylsilane (TMS) and were referenced to the signal of TMS (0 ppm). ${ }^{13} \mathrm{C}$ NMR spectra were reported in ppm relative to residual $\mathrm{CHCl}_{3}(77.00 \mathrm{ppm})$. Coupling constants, $J$, are reported in hertz $(\mathrm{Hz}) .{ }^{19} \mathrm{~F}$ and ${ }^{31} \mathrm{P}$ NMR spectra were also collected on Bruker AVANCE III $400 \mathrm{MHz}$ spectrometers at room temperature. Melting points were determined on a microscopic apparatus. IR spectra were collected using BrukerTENSOR 27 spectrometer and Agilent Technologies Cary 630 FTIR, and only major peaks were reported in $\mathrm{cm}^{-1}$. HRMS was performed on Bruker Apex II FT-ICR mass instrument (ESI) and waters GCT Premier TOFMS (EI). GC analysis was performed on Thermo Scientific TRACE 1300. GC-MS data were collected on Thermo Scientific TRACE DSQ GC-MS. The X-RAY was measured on Agilent SUPERNOVA. Thinlayer chromatography was carried out using XINNUO SGF254 TLC plates. Flash chromatography was performed using XINNUO silica gel (200-300 mesh). 


\section{Synthesis of ligands}

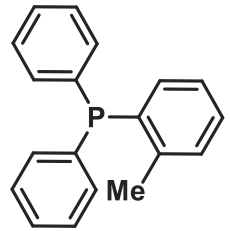

L6

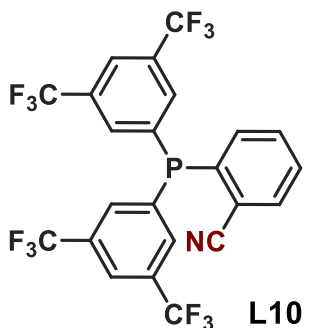

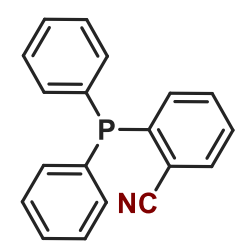

L7

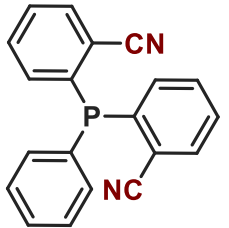

L11

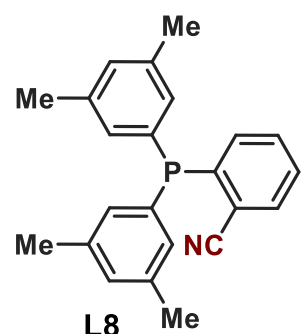

L8

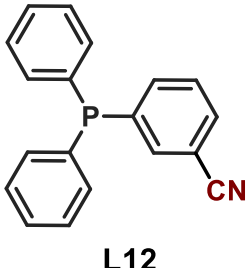

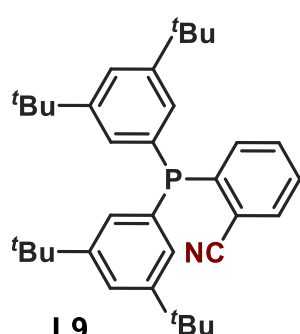

L9

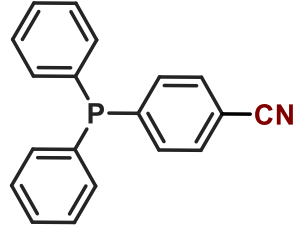

L13

Ligands L6, L7, L13 are commercially available. Ligands $\mathbf{L 1 1}^{2}, \mathbf{L 1 2}^{\mathbf{3}}$ are known compounds and were prepared according to the literature procedure. The preparation of new ligands L8-L10, and their characterization data are provided as follows.

\section{General Procedure A}<smiles>[R]c1cc([R])cc(P(c2cc([R])cc([R])c2)c2cc([R])cc([R])c2)c1</smiles>

Step 1, synthesis of $\mathbf{E t}_{2} \mathbf{N P C l}_{2}$ : To a solution of phosphorous trichloride (8.7 mL, 100 mmol $)$ in dry diethyl ether $(100 \mathrm{~mL})$ at $-78^{\circ} \mathrm{C}$ was dropwise added diethylamine $(20.7$ $\mathrm{mL}, 200 \mathrm{mmol}$ ) under argon atmosphere (the reaction was highly exothermic). The reaction mixture was stirred at $-78{ }^{\circ} \mathrm{C}$ for 1 hour and then at room temperature for 17 hours. The resulting precipitate was quickly filtered and washed with dry ether $(50 \mathrm{~mL})$. The filtrate was evaporated to dryness and the residue was distilled to give $\mathrm{Et}_{2} \mathrm{NPCl}_{2}$ (moisture sensitive).

Step 2: To a solution of $\mathrm{Ar}-\mathrm{Br}(10.5 \mathrm{mmol})$ in anhydrous $\mathrm{Et}_{2} \mathrm{O}(50 \mathrm{~mL})$ was dropwise 
added $n$-BuLi (4.2 $\mathrm{mL}, 10.5 \mathrm{mmol}, 2.5 \mathrm{M}$ in hexane) at $0{ }^{\circ} \mathrm{C}$. The reaction mixture was stirred at the same temperature for 4 hours. $\mathrm{Et}_{2} \mathrm{NPCl}_{2}(0.86 \mathrm{~g}, 5.0 \mathrm{mmol})$ was added slowly, and the reaction mixture was stirred at $0{ }^{\circ} \mathrm{C}$ for $10 \mathrm{~min}$, resulting in a suspension. Addition of $\mathrm{HCl}(2.9 \mathrm{~mL}, 11.6 \mathrm{mmol}, 4.0 \mathrm{M}$ in 1,4-dioxane) gave rise to a thick white suspension. It was filtered through a pad of celite and the filtrate was concentrated under the reduced pressure. The residue was further concentrated in high vacuum for one day to afford $\mathrm{Ar}_{2} \mathrm{PCl}$ in quantitative yield.

Step 3: To a flame dried schlenk tube charged with 2-bromobenzonitrile (5 mmol) and THF (25 mL) was dropwise added $n$-BuLi ( $2 \mathrm{~mL}, 5 \mathrm{mmol}, 2.5 \mathrm{M}$ solution in hexane) at $-78{ }^{\circ} \mathrm{C}$ under argon atmosphere. After stirring at the same temperature for 1 hour, a solution of $\mathrm{Ar}_{2} \mathrm{PCl}(\sim 5 \mathrm{mmol})$ in THF $(5 \mathrm{~mL})$ was slowly added. The reaction mixture was stirred at $-78^{\circ} \mathrm{C}$ for additional 1 hour and then at room temperature for 12 hours. The reaction was quenched with water and extracted with ethyl acetate $(3 \times 30 \mathrm{~mL})$. The combined organic layers was washed with brine, dried over anhydrous $\mathrm{Na}_{2} \mathrm{SO}_{4}$, filtered, and concentrated under reduced pressure. The residue was purified by flash column chromatography on silica gel to afford ligands L8 and L9.

\section{2-(Bis(3,5-dimethylphenyl)phosphanyl)benzonitrile (L8)}

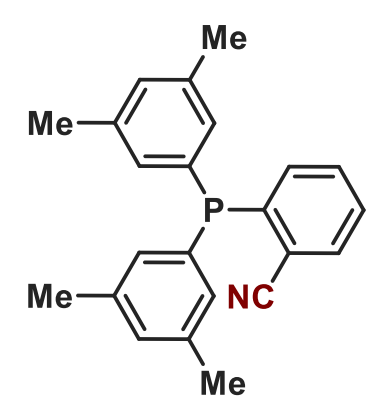

This compound was prepared from 1-bromo-3,5dimethylbenzene (1.9 g, $10.5 \mathrm{mmol})$ and 2-bromobenzonitrile $(0.9 \mathrm{~g}, 5.0 \mathrm{mmol})$ according to the General procedure A. $1.4 \mathrm{~g}$ (82\% yield) from 2-bromobenzonitrile, white solid, mp: $136-138^{\circ} \mathrm{C}$

${ }^{1}$ H NMR (400 MHz, CDCl 3 ): $\delta 7.70$ (ddd, $\left.J=8.0,4.0,0.8 \mathrm{~Hz}, 1 \mathrm{H}\right), 7.47$ (td, $J=8.0$, $4.0 \mathrm{~Hz}, 1 \mathrm{H}), 7.40(\mathrm{td}, J=8.0,4.0 \mathrm{~Hz}, 1 \mathrm{H}), 7.05$ (ddd, $J=8.0,4.0,0.8 \mathrm{~Hz}, 1 \mathrm{H}), 7.01$ (s, $2 \mathrm{H}), 6.91(\mathrm{~s}, 2 \mathrm{H}), 6.89$ (s, $2 \mathrm{H}), 2.27$ (s, $12 \mathrm{H})$.

${ }^{13}$ C NMR (100 MHz, CDCl $): \delta 143.5\left(\mathrm{~d}, J_{\mathrm{CP}}=20.0 \mathrm{~Hz}, \mathrm{C}\right), 138.1\left(\mathrm{~d}, J_{\mathrm{CP}}=8.0 \mathrm{~Hz}, 4\right.$ $\mathrm{CH}), 134.3\left(\mathrm{~d}, J_{\mathrm{CP}}=9.0 \mathrm{~Hz}, 2 \mathrm{C}\right), 133.6\left(\mathrm{~d}, J_{\mathrm{CP}}=5.0 \mathrm{~Hz}, \mathrm{CH}\right), 133.5(\mathrm{~s}, \mathrm{CH}), 132.3(\mathrm{~s}$, 
$\mathrm{CH}), 131.6\left(\mathrm{~d}, J_{\mathrm{CP}}=20.0 \mathrm{~Hz}, 4 \mathrm{CH}\right), 131.2(\mathrm{~s}, 2 \mathrm{CH}), 128.6(\mathrm{~s}, \mathrm{CH}), 117.8\left(\mathrm{~d}, J_{\mathrm{CP}}=3.6\right.$ $\mathrm{Hz}, \mathrm{CN}), 117.77\left(\mathrm{~d}, J_{\mathrm{CP}}=32.9 \mathrm{~Hz}, \mathrm{C}\right), 21.3\left(\mathrm{~s}, 4 \mathrm{CH}_{3}\right)$.

${ }^{31}$ P NMR (161 MHz, CDCl3): $\delta$-8.6.

IR (neat, $\mathbf{c m}^{-1}$ ): 2957, 2379, 2221, 1718, 1510, 1458, 1091, 846, 692.

HRMS (ESI): $[\mathrm{M}+\mathrm{H}]^{+}$calcd for $\mathrm{C}_{23} \mathrm{H}_{23} \mathrm{NP} 344.1563$, found: 344.1560 .

\section{2-(Bis(3,5-di-tert-butylphenyl)phosphanyl)benzonitrile (L9)}

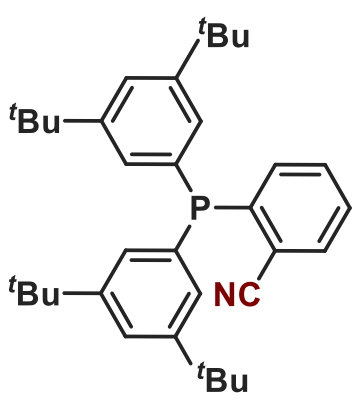

This compound prepared from 1-bromo-3,5-di-tertbutylbenzene (3.77 g, $14 \mathrm{mmol})$ and 2-bromobenzonitrile (0.9 $\mathrm{g}, 5.0 \mathrm{mmol}$ ) according to the General procedure A.

$1.8 \mathrm{~g}$ (70\% yield) from 2-bromobenzonitrile, white solid, mp: $127-129^{\circ} \mathrm{C}$.

${ }^{1}$ H NMR (400 MHz, CDCl3): $\delta$ 7.72-7.69 (m, 1 H), 7.50-7.46 (m, $1 \mathrm{H}), 7.41-7.38$ (m, $3 \mathrm{H}), 7.12-7.10(\mathrm{~m}, 4 \mathrm{H}), 7.08-7.05$ (m, $1 \mathrm{H}), 1.24$ (s, $36 \mathrm{H})$.

${ }^{13} \mathrm{C}$ NMR (100 MHz, CDCl3): $\delta 150.8\left(\mathrm{~d}, J_{\mathrm{CP}}=7.0 \mathrm{~Hz}, 4 \mathrm{C}\right), 144.4\left(\mathrm{~d}, J_{\mathrm{CP}}=20.0 \mathrm{~Hz}\right.$, C), $133.8\left(\mathrm{~d}, J_{\mathrm{CP}}=8.0 \mathrm{~Hz}, 2 \mathrm{C}\right), 133.5\left(\mathrm{~d}, J_{\mathrm{CP}}=9.0 \mathrm{~Hz}, \mathrm{CH}\right), 133.3(\mathrm{~s}, \mathrm{CH}), 132.0(\mathrm{~s}$, $\mathrm{CH}), 128.5(\mathrm{~s}, \mathrm{CH}), 128.2\left(\mathrm{~d}, J_{\mathrm{CP}}=20.0 \mathrm{~Hz}, 4 \mathrm{CH}\right), 123.1(\mathrm{~s}, 2 \mathrm{CH}), 118.0\left(\mathrm{~d}, J_{\mathrm{CP}}=\right.$ $33.0 \mathrm{~Hz}, \mathrm{C}), 117.8\left(\mathrm{~d}, J_{\mathrm{CP}}=3.0 \mathrm{~Hz}, \mathrm{CN}\right), 34.9(\mathrm{~s}, 4 \mathrm{C}), 31.3\left(\mathrm{~s}, 12 \mathrm{CH}_{3}\right)$.

${ }^{31}$ P NMR (161 MHz, CDCl3): $\delta-6.5$.

IR (neat, $\mathbf{c m}^{-1}$ ): 2957, 2379, 2221, 1718, 1510, 1458, 1091, 846, 692.

HRMS (ESI): $[\mathrm{M}+\mathrm{H}]{ }^{+}$calcd for $\mathrm{C}_{35} \mathrm{H}_{47} \mathrm{NP} 512.3441$, found: 512.3442 .

2-(Bis(3,5-bis(trifluoromethyl)phenyl)phosphanyl)benzonitrile (L10)
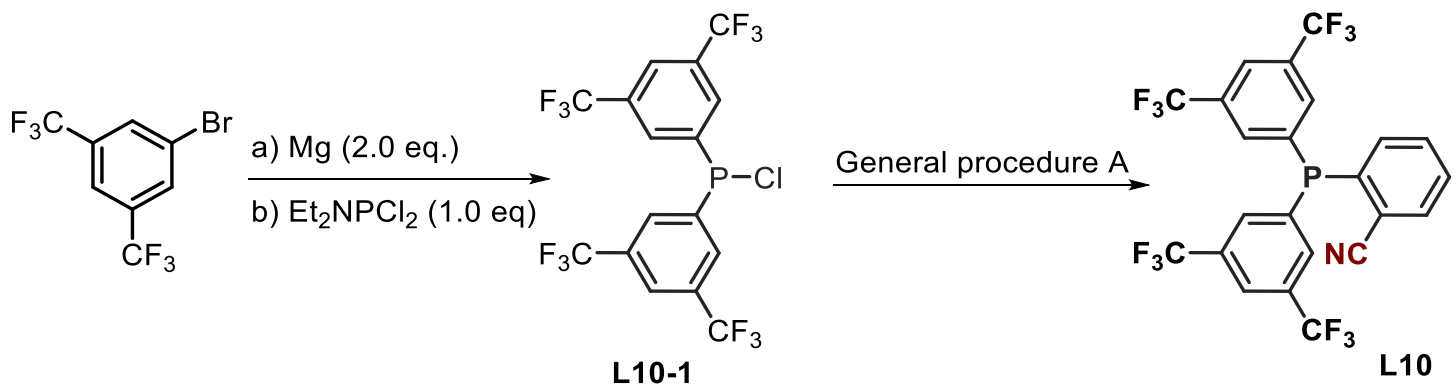
Step 1: To a mixture of magnesium turnings (480 mg, $20 \mathrm{mmol})$ in THF (5 mL) was added a solution of 1,3-bis(trifluoromethyl)-5-bromobenzene $(5.8 \mathrm{~g}, 20 \mathrm{mmol})$ in THF $(20 \mathrm{~mL})$ at $0{ }^{\circ} \mathrm{C}$. The reaction mixture was stirred at room temperature for 1 hour, resulting in the formation of a brown solution. It was then cooled down to $0{ }^{\circ} \mathrm{C}$, and a solution of $\mathrm{Et}_{2} \mathrm{NPCl}_{2}(1.7 \mathrm{~g}, 10.0 \mathrm{mmol})$ in THF $(10 \mathrm{~mL})$ was added dropwise. The reaction mixture was warmed to room temperature and stirred overnight. The solvent was removed in vacuum. The residue was dissolved in hexane, filtered through celite, and concentrated. A solution of $\mathrm{HCl}$ in 1, 4-dioxane $(5 \mathrm{~mL}, 20 \mathrm{mmol}, 4.0 \mathrm{M}$ in 1, 4dioxane,) was dropwise added at room temperature. The reaction mixture was stirred for additional 2 hours, and the formed white precipitate of amine hydrochloride was filtered. The filtrate was concentrated in vacuum to afford chlorophosphine L10-1 as a white solid.

Step 2: Compound L10 was prepared from bis(3,5-bis(trifluoromethyl)phenyl) chlorophosphane (L10-1, $3.0 \mathrm{~g}, 6.0 \mathrm{mmol})$ and 2-bromobenzonitrile (1.1 g, $6.0 \mathrm{mmol})$ according to the General procedure A.

$1.9 \mathrm{~g}$ (56\% yield) from 2-bromobenzonitrile, white solid, mp: 98-100 ${ }^{\circ} \mathrm{C}$.

${ }^{1}$ H NMR (400 MHz, CDCl 3 ): $\delta 7.97$ (s, 2 H), 7.88-7.85 (m, 1 H), 7.74 (s, 2 H), 7.72 (s, $2 \mathrm{H})$, 7.69-7.61 (m, $2 \mathrm{H})$, 7.07-7.04 (m, $1 \mathrm{H})$.

${ }^{13}$ C NMR (100 MHz, CDCl3): $\delta 137.9\left(\mathrm{~d}, J_{\mathrm{CP}}=18.0 \mathrm{~Hz}, \mathrm{C}\right), 136.9\left(\mathrm{~d}, J_{\mathrm{CP}}=17.0 \mathrm{~Hz}\right.$, $4 \mathrm{CH}), 134.6\left(\mathrm{~d}, J_{\mathrm{CP}}=5.0 \mathrm{~Hz}, \mathrm{CH}\right), 133.5\left(\mathrm{~d}, J_{\mathrm{CP}}=12.0 \mathrm{~Hz}, 2 \mathrm{C}\right), 133.48\left(\mathrm{~d}, J_{\mathrm{CP}}=4.0\right.$ $\mathrm{Hz}, \mathrm{CH}), 133.3\left(\mathrm{~d}, J_{\mathrm{CP}}=4.0 \mathrm{~Hz}, \mathrm{CH}\right), 132.6\left(\mathrm{qd}, J_{\mathrm{CF}}=34.0 \mathrm{~Hz}, J_{\mathrm{CP}}=7.0 \mathrm{~Hz}, 4 \mathrm{C}\right)$, $130.9(\mathrm{~s}, 1 \mathrm{CH}), 124.2\left(\mathrm{q}, J_{\mathrm{CF}}=4.0 \mathrm{~Hz}, 2 \mathrm{CH}\right), 122.8\left(\mathrm{q}, J_{\mathrm{CF}}=272 \mathrm{~Hz}, 4 \mathrm{CF}_{3}\right), 118.4$ $\left(\mathrm{d}, J_{\mathrm{CP}}=34.2 \mathrm{~Hz}, \mathrm{C}\right), 116.8\left(\mathrm{~d}, J_{\mathrm{CP}}=4.4 \mathrm{~Hz}, \mathrm{CN}\right)$.

${ }^{31}$ P NMR (161 MHz, $\left.\mathrm{CDCl}_{3}\right): \delta-8.1$.

${ }^{19}$ F NMR (376 MHz, CDCl3): $\delta-63.0$.

IR (neat, $\left.\mathbf{c m}^{-1}\right)$ : 2954, 2378, 2310, 1354, 1279, 1176, 1124, 897, 681.

HRMS (ESI): $[\mathrm{M}+\mathrm{H}]^{+}$calcd for $\mathrm{C}_{23} \mathrm{H}_{11} \mathrm{~F}_{12} \mathrm{NP} 560.0432$, found: 560.0429 . 


\section{Preparation of Starting Materials}

\subsection{Synthesis of 1,3-Dienes}<smiles>C=C/C=C(\C)CCC=C(C)C</smiles>

$1 a$<smiles>C=C/C=C/CCCC</smiles>

$1 \mathrm{~b}$<smiles>C=C/C=C/CCCCCCC</smiles>

1c<smiles>C=CC=CC1CCCCC1</smiles>

1d

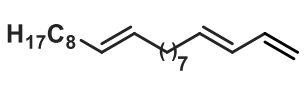

$1 e$

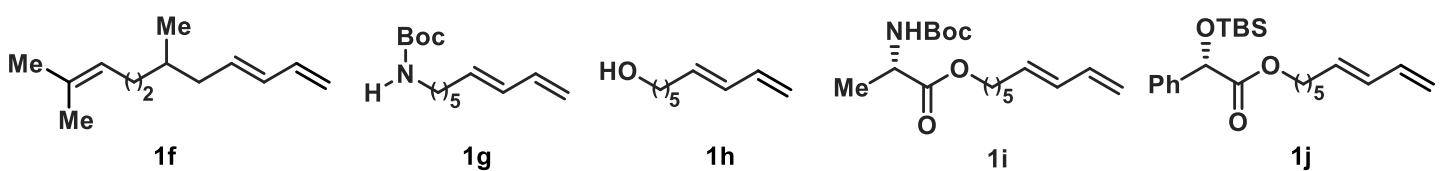<smiles>C=CC=CC=CCCCCCOC(=O)C1CCCC1C1CCC1</smiles><smiles>C=C/C=C/c1ccc(OC)cc1</smiles>

$1 \mathrm{n}$

10<smiles>C=C/C=C/c1ccc(O)cc1</smiles>

$1 \mathrm{p}$<smiles>C=C/C=C/c1ccc(C)cc1</smiles>

$1 q$<smiles>C=C/C=C/c1ccc(C(C)=O)cc1</smiles>

$1 \mathrm{r}$

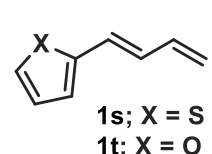<smiles>C=C/C=C(\C)c1ccccc1</smiles>

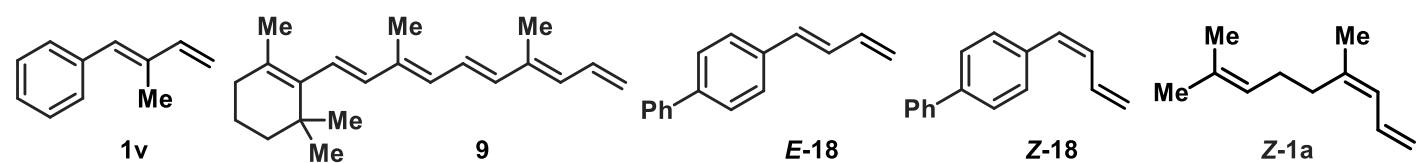

Compounds 11 and $\mathbf{1 m}$ are commercially available. Known compounds $\mathbf{1 a}^{4}, \mathbf{1 b}^{5}, \mathbf{1 c}^{5}$, $1 d^{6}, \mathbf{1 f}^{7}, \mathbf{1 h}^{8}, \mathbf{1 n}^{6}, 1 \mathbf{1}^{6}, \mathbf{1} \mathbf{p}^{9}, 1 \mathbf{q}^{10}, \mathbf{1 r}^{9}, \mathbf{1 s}^{6}, \mathbf{1 t}^{6}, \mathbf{1 u}^{11}, \mathbf{1 v}^{9}, \mathbf{9}^{12}, \boldsymbol{E}-\mathbf{1 8} \mathbf{8}^{13}, Z-1 \mathbf{a}^{14}$ were prepared according the literature procedure. The preparation of new compounds and their characterization data are provided as follows.

\section{General procedure B:}

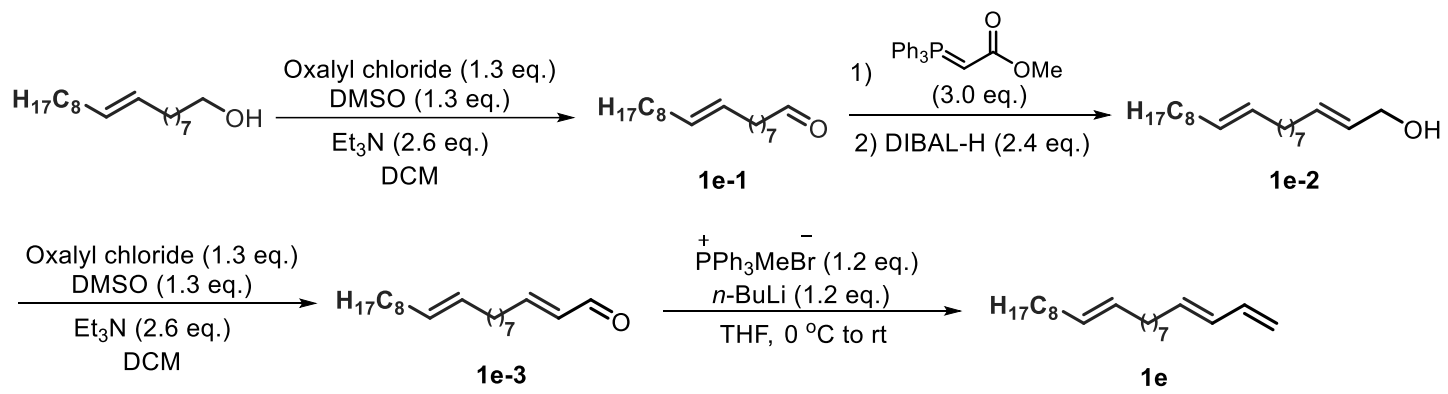

Step 1: To a solution of oxalyl chloride (3.3 g, $26.0 \mathrm{mmol})$ in anhydrous DCM (30 mL) was dropwise added a solution of DMSO $(1.9 \mathrm{~mL}, 26.0 \mathrm{mmol}, 1.5 \mathrm{M}$ in anhydrous 
DCM) at $-78^{\circ} \mathrm{C}$. After stirring at the same temperature for $30 \mathrm{~min}$, a solution of alcohol (5.4 g, $20.0 \mathrm{mmol})$ in anhydrous DCM $(100 \mathrm{~mL})$ was added dropwise. It was stirred at $-78^{\circ} \mathrm{C}$ for $30 \mathrm{~min}$, and $\mathrm{Et}_{3} \mathrm{~N}(7.2 \mathrm{~mL}, 52.0 \mathrm{mmol})$ was then added. The reaction mixture was allowed to warm to $0{ }^{\circ} \mathrm{C}$ and stirred for additional 1 hour. The reaction was quenched with water and extracted with DCM for 3 times. The combined organic layers was washed with saturated brine, dried over anhydrous $\mathrm{Na}_{2} \mathrm{SO}_{4}$, filtered, and concentrated in vacuum. The residue was purified by flash column chromatography on silica gel to afford aliphatic aldehyde in $77 \%$ yield $(4.1 \mathrm{~g})$.

Step 2: To a solution of aliphatic aldehyde ( $4.1 \mathrm{~g}, 15.3 \mathrm{mmol})$ in anhydrous toluene (75 $\mathrm{mL}$ ) was added methyl (triphenylphosphoranylidene)acetate $(15.3 \mathrm{~g}, 45.9 \mathrm{mmol})$ at room temperature. The mixture was stirred at $80{ }^{\circ} \mathrm{C}$ for 4 hours, then cooled down to room temperature, and diluted with petroleum ether. The formed precipitate was filtered and the filtrate was concentrated in vacuum. The residue was purified by flash column chromatography on silica gel to afford acrylates in quantitative yield.

Step 3: To a solution of acrylates $(5.0 \mathrm{~g}, 15.0 \mathrm{mmol})$ in anhydrous toluene $(80 \mathrm{~mL})$ was dropwise added DIBAL-H (24.0 mL, 1.5 M in toluene, 2.4 equiv) at $-78^{\circ} \mathrm{C}$ for $20 \mathrm{~min}$. The reaction mixture was stirred at the same temperature for 4 hours. The reaction was quenched by slow addition of ethyl acetate at $-78{ }^{\circ} \mathrm{C}$. It was stirred at the same temperature for 20 min and warmed up to room temperature. The reaction mixture was carefully poured into a rapidly stirred mixture of saturated aqueous Rochelle's salt (potassium sodium tartrate tetrahydrate) and the resultant cloudy mixture was stirred vigorously until the organic layer became clear. It was extracted with ethyl acetate for 3 times, and the combined organic layers was dried over anhydrous $\mathrm{Na}_{2} \mathrm{SO}_{4}$. The solvent was removed in vacuum, and the residue was purified by flash column chromatography on silica gel to afford the allylic alcohol in $89 \%$ yield (3.9 g).

Step 4: The enal was prepared according to the procedure used in Step 1 in $67 \%$ yield $(2.6 \mathrm{~g})$.

Step 5: To a suspension of methyltriphenylphosphonium bromide (3.8 g, $10.7 \mathrm{mmol})$ in anhydrous THF $(50.0 \mathrm{~mL})$ was added a solution of $n$-BuLi $(4.3 \mathrm{~mL}, 10.7 \mathrm{mmol})$ in 
hexane $(2.5 \mathrm{M})$ at $0{ }^{\circ} \mathrm{C}$ under argon atmosphere. After stirring at $0{ }^{\circ} \mathrm{C}$ until all phosphonium bromide dissolved, a solution of enal (2.6 g, $8.9 \mathrm{mmol})$ in anhydrous THF $(10 \mathrm{~mL})$ was added slowly. The reaction mixture was warmed to room temperature and stirred overnight. A saturated solution of $\mathrm{NH}_{4} \mathrm{Cl}$ was added, and the mixture was extracted with $\mathrm{Et}_{2} \mathrm{O}$. The combined organic layers was washed with brine, dried over anhydrous $\mathrm{Na}_{2} \mathrm{SO}_{4}$, filtered, and concentrated in vacuum. The residue was purified by flash column chromatography on silica gel to afford the target diene in $73 \%$ yield (1.9 g).

\section{$(3 E, 12 E)$-pentadeca-1,3,12-triene (1e)}

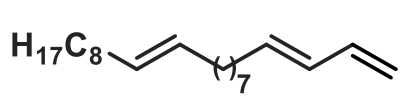

This compound was prepared from oleyl alcohol $(6.3 \mathrm{~mL}$, $20.0 \mathrm{mmol}$ ) according to the General procedure B.

$1.9 \mathrm{~g}$ (33\% yield) for five steps, colorless oil.

${ }^{1}$ H NMR (400 MHz, CDCl 3 ): $\delta 6.31$ (dt, $\left.J=16.0,8.0 \mathrm{~Hz}, 1 \mathrm{H}\right), 6.04(\mathrm{dd}, J=16.0,8.0$ $\mathrm{Hz}, 1 \mathrm{H}), 5.74-5.67(\mathrm{~m}, 1 \mathrm{H}), 5.39-5.31(\mathrm{~m}, 2 \mathrm{H}), 5.08$ (d, $J=16.0 \mathrm{~Hz}, 1 \mathrm{H}), 4.95$ (d, $J$ $=12.0 \mathrm{~Hz}, 1 \mathrm{H}), 2.10-1.99(\mathrm{~m}, 6 \mathrm{H}), 1.40-1.27(\mathrm{~m}, 22 \mathrm{H}), 0.88(\mathrm{t}, J=8.0 \mathrm{~Hz}, 3 \mathrm{H})$.

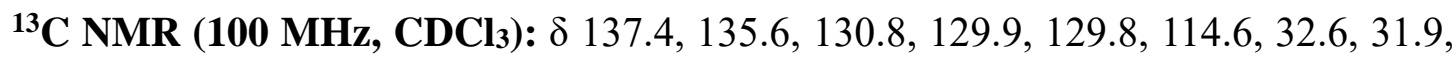
29.8, 29.7, 29.5, 29.4, 29.3 (2 C), 29.23, 29.2 (2 C), 27.21, 27.2, 22.7, 14.1.

IR (neat, $\mathbf{c m}^{-1}$ ): 2924, 2854, 1650, 1462, 1375, 1002, 968, 895, 722.

HRMS (EI): [M] ${ }^{+}$calcd for $\mathrm{C}_{21} \mathrm{H}_{38} 290.2974$, found: 290.2970 .

tert-Butyl (E)-nona-6,8-dien-1-ylcarbamate (1g)

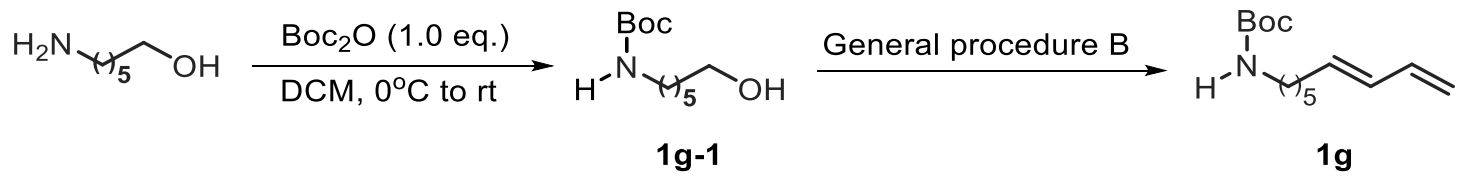

To a stirred solution of 6-amino hexanol (5.9 g, $50.0 \mathrm{mmol})$ in DCM (1 M, $50 \mathrm{~mL})$ was slowly added $\mathrm{Boc}_{2} \mathrm{O}(10.9 \mathrm{~g}, 50 \mathrm{mmol})$ at $0{ }^{\circ} \mathrm{C}$. The reaction mixture was stirred at room temperature for overnight. The solvent was removed in vacuum, and the residue was purified by flash column chromatography on silica gel to afford tert-butyl (6- 
hydroxyhexyl)carbamate 1g-1 in quantitative yield.

Diene $1 \mathrm{~g}$ was prepared from $\mathbf{1 g - 1}(10.9 \mathrm{~g}, \sim 50.0 \mathrm{mmol})$ according to the General procedure B. $5.4 \mathrm{~g}$ (45\% yield) for six steps, colorless oil.

${ }^{1}$ H NMR (400 MHz, CDCl3): $\delta 6.30(\mathrm{dt}, J=16.0,12.0 \mathrm{~Hz}, 1 \mathrm{H}), 6.04(\mathrm{dd}, J=16.0$, $12.0 \mathrm{~Hz}, 1 \mathrm{H}), 5.72-5.65(\mathrm{~m}, 1 \mathrm{H}), 5.09(\mathrm{~d}, J=16.8 \mathrm{~Hz}, 1 \mathrm{H}), 4.96(\mathrm{~d}, J=12.0 \mathrm{~Hz}, 1$ H), 4.50 (br, 1 H), 3.13-3.08 (m, 2 H), 2.11-2.06 (m, 2 H), 1.51-1.38 (m, 13 H), 1.36$1.28(\mathrm{~m}, 2 \mathrm{H})$.

${ }^{13}$ C NMR (100 MHz, CDCl $): \delta 155.9,137.2,135.0,131.0,114.7,78.9,40.3,32.3$, $29.9,28.7,28.3,26.3$.

IR (neat, cm$^{-1}$ ): 2976, 2931, 2858, 1695, 1526, 1366, 1250, 1174, 951, 869, 731.

HRMS (ESI): $[\mathrm{M}+\mathrm{Na}]^{+}$calcd for $\mathrm{C}_{14} \mathrm{H}_{25} \mathrm{NNaO}_{2} 262.1778$, found: 262.1775 .

\section{(E)-nona-6,8-dien-1-ol (1h)}

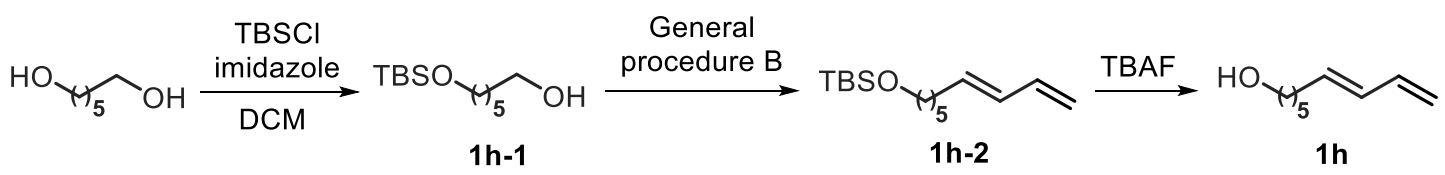

To a cooled suspension of 1,6-hexanediol (11.8 g, $100.0 \mathrm{mmol})$ and TBSCl (15.1 g, $100.0 \mathrm{mmol})$ in anhydrous DCM $(100 \mathrm{~mL})$ was added imidazole $(6.8 \mathrm{~g}, 100.0 \mathrm{mmol})$. The reaction mixture was stirred at room temperature for overnight and then diluted with DCM $(100 \mathrm{~mL})$. It was washed with water $(3 \times 60 \mathrm{~mL})$, dried over anhydrous $\mathrm{Na}_{2} \mathrm{SO}_{4}$, filtered, and concentrated in vacuum. The residue was purified by flash column chromatography on silica gel to afford $\mathbf{1 h - 1}$ in 57\% yield (13.2 g).

Diene 1h-2 (4.9 g, 33.8\% yield) was prepared from 1h-1 (13.2 g, $56.8 \mathrm{mmol})$ according to the General procedure B.

To a solution of diene $\mathbf{1 h}-\mathbf{2}(4.9 \mathrm{~g}, 19.3 \mathrm{mmol})$ in THF $(32.0 \mathrm{~mL})$ was added TBAF 3 $\mathrm{H}_{2} \mathrm{O}(12.2 \mathrm{~g}, 38.6 \mathrm{mmol})$ at room temperature. After stirring for 4 hours, the reaction mixture was diluted with water and extracted with $\mathrm{Et}_{2} \mathrm{O}(3 \times 60 \mathrm{~mL})$. The combined organic layers was washed with brine and dried over anhydrous $\mathrm{Na}_{2} \mathrm{SO}_{4}$. The solvent was removed in vacuum and the residue was purified by flash column chromatography 
on silica gel to afford the product $\mathbf{1 h}$.

${ }^{1} \mathrm{H}$ NMR and ${ }^{13} \mathrm{C}$ NMR data are consistent with those reported in reference 8.

$2.2 \mathrm{~g}$ (16\% yield) for seven steps, colorless oil.

${ }^{1}$ H NMR (400 MHz, CDCl3): $\delta 6.31(\mathrm{dt}, J=16.0,12.0 \mathrm{~Hz}, 1 \mathrm{H}), 6.05(\mathrm{dd}, J=16.0$, $12.0 \mathrm{~Hz}, 1 \mathrm{H}), 5.74-5.66(\mathrm{~m}, 1 \mathrm{H}), 5.09$ (d, $J=16.0 \mathrm{~Hz}, 1 \mathrm{H}), 4.96(\mathrm{~d}, J=12.0 \mathrm{~Hz}, 1$ H), $3.64(\mathrm{t}, J=6.8 \mathrm{~Hz}, 2 \mathrm{H}), 2.13-2.07(\mathrm{~m}, 2 \mathrm{H}), 1.61-1.54(\mathrm{~m}, 2 \mathrm{H}), 1.49$ (br, $1 \mathrm{H})$, 1.45-1.35 (m, $4 \mathrm{H})$.

${ }^{13}$ C NMR (100 MHz, CDCl3): $\delta$ 137.4, 135.3, 131.2, 114.9, 63.0, 32.7, 32.6, 29.1, 25.4.

(E)-nona-6,8-dien-1-yl (tert-butoxycarbonyl)-D-alaninate (1i)

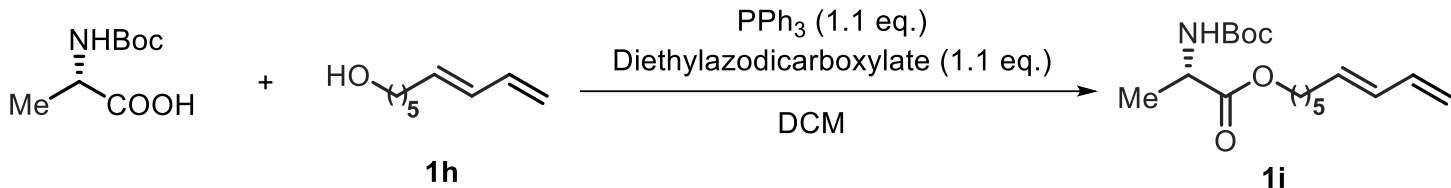

To a solution of BOC- $L$-alanine $(567 \mathrm{mg}, 3.0 \mathrm{mmol})$, alcohol (462 mg, $3.3 \mathrm{mmol})$ and $\mathrm{PPh}_{3}(865 \mathrm{mg}, 3.3 \mathrm{mmol})$ in DCM $(15 \mathrm{~mL})$ was added diethylazodicarboxylate (575 $\mathrm{mg}, 3.3 \mathrm{mmol}$ ) for $15 \mathrm{~min}$. After stirring at room temperature for 14 hours, the reaction mixture was diluted with DCM $(30 \mathrm{~mL})$ and then washed with saturated $\mathrm{NaHCO}_{3}(3 \times$ $15 \mathrm{~mL}$ ). The solvent was removed in vacuum. The residue was dissolved in $\mathrm{Et}_{2} \mathrm{O}$ (50 $\mathrm{mL}$ ) and placed in a refrigerator at $0{ }^{\circ} \mathrm{C}$ to precipitate the phosphine oxide. It was filtered, and the solvent was removed in vacuum. The residue was purified by flash column chromatography on silica gel to afford the target product in $89 \%$ yield $(830 \mathrm{mg})$ as a colorless oil.

${ }^{1}$ H NMR (400 MHz, CDCl3): $\delta 6.31(\mathrm{dt}, J=16.0,12.0 \mathrm{~Hz}, 1 \mathrm{H}), 6.05(\mathrm{dd}, J=16.0$, 12.0 Hz, $1 \mathrm{H}), 5.72-5.65$ (m, $1 \mathrm{H}), 5.11-5.05$ (m, $2 \mathrm{H}), 4.96(\mathrm{~d}, J=12.0 \mathrm{~Hz}, 1 \mathrm{H}), 4.32-$ 4.27 (m, 1 H), 4.18-4.08 (m, 2 H), 2.12-2.07 (m, 2 H), 1.69-1.60 (m, 2 H), 1.45-1.32 (m, $16 \mathrm{H})$.

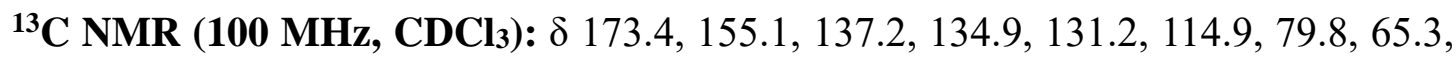
49.2, 32.3, 28.7, 28.4, 28.3, 25.3, 18.8 . 
IR (neat, $\mathbf{c m}^{-1}$ ): 2977, 2932, 1716, 1510, 1454, 1391, 1345, 1166, 1068, 1004, 896, 781.

HRMS (ESI): $[\mathrm{M}+\mathrm{Na}]^{+}$calcd for $\mathrm{C}_{17} \mathrm{H}_{29} \mathrm{NNaO}_{4}$ 334.1989, found: 334.1986 .

\section{(E)-nona-6,8-dien-1-yl (S)-2-((tert-butyldimethylsilyl)oxy)-2-phenylacetate $(1 \mathrm{j})$}

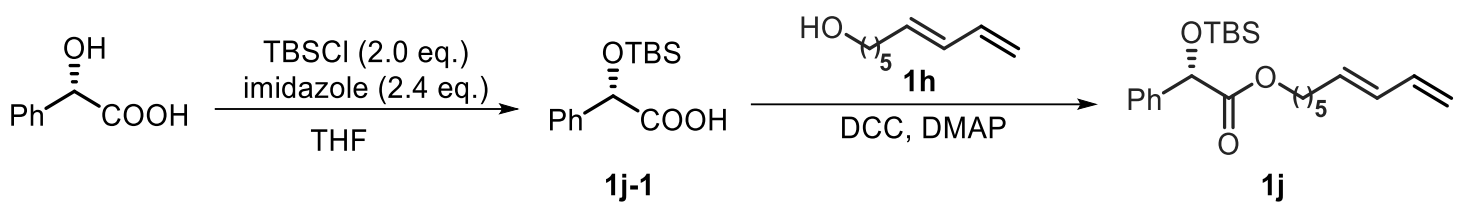

Step 1: To a solution of L-(+)-mandelic acid (760 mg, $5.0 \mathrm{mmol})$ in THF (10 mL) was added $\mathrm{TBSCl}(1.5 \mathrm{~g}, 10.0 \mathrm{mmol})$ and imidazole $(816 \mathrm{mg}, 12 \mathrm{mmol})$ at $0{ }^{\circ} \mathrm{C}$. The reaction mixture was stirred at $0{ }^{\circ} \mathrm{C}$ for $30 \mathrm{~min}$ and then at room temperature for 12 hours. The solution was filtered, and the filtrate was concentrated in vacuum. The resulting oil was dissolved in $\mathrm{NaOH}$ aqueous solution $(15 \mathrm{~mL}, 1 \mathrm{M})$ and stirred for 1.5 hours. The reaction mixture was diluted with $\mathrm{H}_{2} \mathrm{O}(10 \mathrm{~mL})$ and extracted with $\mathrm{Et}_{2} \mathrm{O}(20 \mathrm{~mL})$. The aqueous layer was acidified with $10 \% \mathrm{HCl}$ until a $\mathrm{PH}$ of 3.5 , and subsequently extracted with $\mathrm{Et}_{2} \mathrm{O}(2 \times 20 \mathrm{~mL})$. The combined organic layers was dried over anhydrous $\mathrm{Na}_{2} \mathrm{SO}_{4}$, filtered, and concentrated in vacuum to afford acid $\mathbf{1 j} \mathbf{j} \mathbf{1}$ in quantitative yield, which was used without further purification.

Step 2: To a solution of the above residue $\mathbf{1 j - 1}$, alcohol $\mathbf{1 h}$ (700 $\mathrm{mg}, 5.0 \mathrm{mmol})$ and 4 dimethylaminopyridine (DMAP, $30.5 \mathrm{mg}, 5 \mathrm{~mol} \%)$ in DCM $(10 \mathrm{~mL})$ at $0{ }^{\circ} \mathrm{C}$ was added dicyclohexylcarbodiimide (DCC, $134 \mathrm{mg}, 3.3 \mathrm{mmol}$ ) in one portion. The reaction mixture was stirred at $0{ }^{\circ} \mathrm{C}$ for $30 \mathrm{~min}$ and then at room temperature overnight. Pentane $(20 \mathrm{~mL})$ was added, and the solution was filtered through a short plug of silica. The filtrate was concentrated in vacuum, and the residue was purified by flash column chromatography on silica gel to afford $1.53 \mathrm{~g}$ (79\% yield for two steps) of the target product $\mathbf{1} \mathbf{j}$ as a colorless oil.

${ }^{1}$ H NMR (400 MHz, CDCl3): $\delta 7.47(\mathrm{~d}, J=8.0 \mathrm{~Hz}, 2 \mathrm{H}), 7.35-7.26(\mathrm{~m}, 3 \mathrm{H}), 6.30(\mathrm{dt}$, $J=16.0,12.0 \mathrm{~Hz}, 1 \mathrm{H}), 6.02(\mathrm{dd}, J=16.0,12.0 \mathrm{~Hz}, 1 \mathrm{H}), 5.69-5.61(\mathrm{~m}, 1 \mathrm{H}), 5.21(\mathrm{~s}$, $1 \mathrm{H}), 5.09(\mathrm{~d}, J=16.0 \mathrm{~Hz}, 1 \mathrm{H}), 4.96(\mathrm{~d}, J=8.0 \mathrm{~Hz}, 1 \mathrm{H}), 4.12-4.03(\mathrm{~m}, 2 \mathrm{H}), 2.05-$ 
2.00 (m, 2 H), 1.61-1.54 (m, 2 H), 1.38-1.31 (m, 2 H), 1.28-1.21 (m, 2 H), 0.92 (s, 9 H),

$0.11(\mathrm{~s}, 3 \mathrm{H}), 0.03$ (s, $3 \mathrm{H})$.

${ }^{13}$ C NMR (100 MHz, CDCl $)$ : $\delta$ 172.2, 139.3, 137.2, 135.0, 131.1, 128.2, 128.0, 126.3, $114.8,74.4,65.0,32.3,28.6,28.3,25.7,25.3,18.3,-5.1,-5.2$.

IR (neat, $\mathbf{c m}^{-1}$ ): 2930, 2856, 2119, 1755, 1452, 1360, 1253, 1171, 1004, 837, 779.

HRMS (ESI): $[\mathrm{M}+\mathrm{Na}]^{+}$calcd for $\mathrm{C}_{23} \mathrm{H}_{36} \mathrm{NaO}_{3} \mathrm{Si} 411.2326$, found: 411.2305.

(E)-nona-6,8-dien-1-yl)-ferrocenecarboxylic acetate (1k)

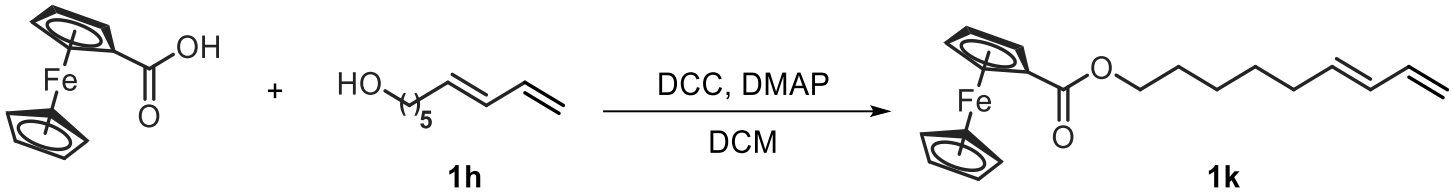

To a solution of alcohol $\mathbf{1 h}(420 \mathrm{mg}, 3.0 \mathrm{mmol})$, ferrocenecarboxylic acid (1.04 g, 4.5 mmol) and DMAP (36.6 mg, $10 \mathrm{~mol} \%)$ in DCM $(20 \mathrm{~mL})$ at $0{ }^{\circ} \mathrm{C}$ was added a solution of DCC (927 mg, $4.5 \mathrm{mmol})$ in DCM (15 mL) for $30 \mathrm{~min}$. The resulting mixture was stirred at room temperature for 24 hours. The reaction was quenched by brine $(30 \mathrm{~mL})$ and extracted with DCM $(3 \times 20 \mathrm{~mL})$. The organic layers was dried over anhydrous $\mathrm{Na}_{2} \mathrm{SO}_{4}$, filtered, and concentrated in vacuum. The residue was purified by flash column chromatography on silica gel to give $610 \mathrm{mg}$ (58\% yield) of the target product $1 \mathbf{k}$ as an orange-red oil.

${ }^{1} \mathbf{H}$ NMR (400 MHz, CDCl3): $\delta 6.31(\mathrm{dt}, J=16.0,12.0 \mathrm{~Hz}, 1 \mathrm{H}), 6.07(\mathrm{dd}, J=16.0$, 12.0 Hz, $1 \mathrm{H}), 5.75-5.68$ (m, $1 \mathrm{H}), 5.09$ (d, $J=16.0 \mathrm{~Hz}, 1 \mathrm{H}), 4.96(\mathrm{~d}, J=12.0 \mathrm{~Hz}, 1$ H), $4.80(\mathrm{t}, J=1.6 \mathrm{~Hz}, 2 \mathrm{H}), 4.38(\mathrm{t}, J=1.6 \mathrm{~Hz}, 2 \mathrm{H}), 4.22-4.19(\mathrm{~m}, 7 \mathrm{H}), 2.16-2.11(\mathrm{~m}$, $2 \mathrm{H}), 1.76-1.70$ (m, $2 \mathrm{H}), 1.53-1.41$ (m, $4 \mathrm{H})$.

${ }^{13}$ C NMR (100 MHz, $\left.\mathbf{C D C l}_{3}\right): \delta 171.7,137.2,135.0,131.2,114.9,71.4,71.2,70.1$, 69.7, 64.1, 32.4, 28.8, 28.7, 25.6 .

IR (neat, cm$^{-1}$ ): 2931, 2855, 2119, 1713, 1460, 1275, 1137, 1003, 952, 894, 821, 775.

HRMS (ESI): $[\mathrm{M}+\mathrm{H}]^{+}$calcd for $\mathrm{C}_{20} \mathrm{H}_{25} \mathrm{FeO}_{2}$ 353.1198, found: 353.1190 . 

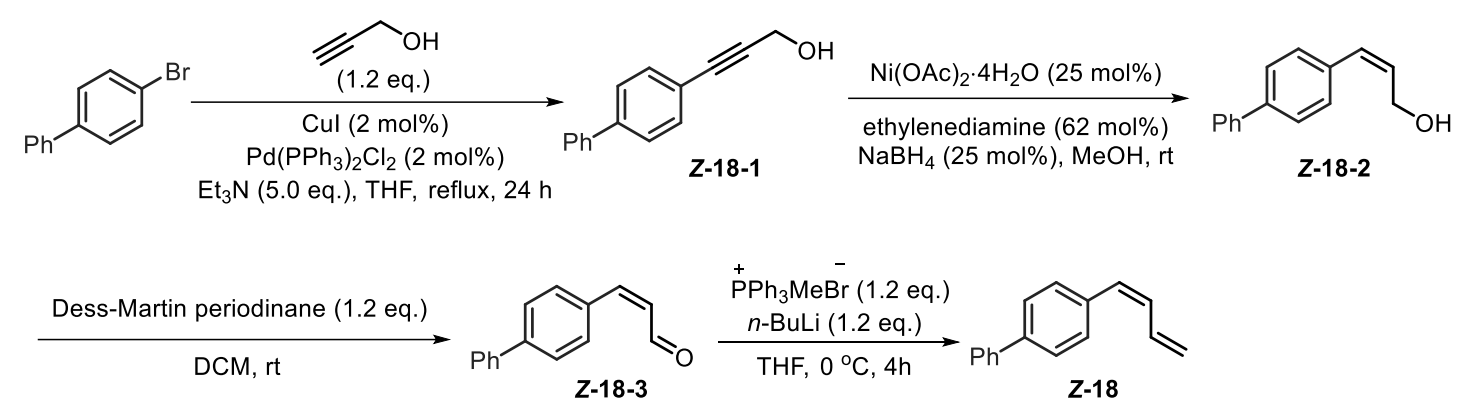

Step 1: Alkyne Z-18-1 was prepared according to the literature procedure. ${ }^{15}$ To a solution of 4-biphenyl bromide (2.3 g, $10 \mathrm{mmol})$, propargyl alcohol (0.7 mL, $12 \mathrm{mmol})$ and $\mathrm{Et}_{3} \mathrm{~N}(7 \mathrm{~mL}, 50 \mathrm{mmol})$ in THF $(20 \mathrm{~mL})$ was added $\mathrm{Pd}\left(\mathrm{Ph}_{3} \mathrm{P}\right)_{2} \mathrm{Cl}_{2}(140 \mathrm{mg}, 2 \mathrm{~mol} \%)$ and $\mathrm{CuI}(38 \mathrm{mg}, 2 \mathrm{~mol} \%)$ under argon atmosphere. The reaction mixture was heated to reflux for 24 hours. $\mathrm{H}_{2} \mathrm{O}(50 \mathrm{~mL})$ and tert-butyl-methyl ether $(50 \mathrm{~mL})$ were added at room temperature, and the two layers were separated. The aqueous layer was extracted with tert-butyl-methyl ether $(2 \times 25 \mathrm{~mL})$. The combined organic layers was washed with brine, dried over anhydrous $\mathrm{MgSO}_{4}$, filtered, and concentrated in vacuum. The residue was purified by flash column chromatography on silica gel to afford alkyne $\mathbf{Z}$ 18-1 in $72 \%$ yield $(1.5 \mathrm{~g})$.

Step 2: Alkene Z-18-2 was prepared according to the literature procedure. ${ }^{16}$

To a suspension of $\mathrm{Ni}(\mathrm{OAc})_{2} \cdot 4 \mathrm{H}_{2} \mathrm{O}(448 \mathrm{mg}, 25 \mathrm{~mol} \%)$ in methanol $(30 \mathrm{~mL}, 0.25 \mathrm{M})$ was added $\mathrm{NaBH}_{4}(68 \mathrm{mg}, 25 \mathrm{~mol} \%)$ at $0{ }^{\circ} \mathrm{C}$ under nitrogen atmosphere. The evolved gas was released through a septum pierced by a needle. The mixture solution was stirred at $0{ }^{\circ} \mathrm{C}$ for $5 \mathrm{~min}$ and at room temperature for $10 \mathrm{~min}$. Ethylenediamine $(0.3 \mathrm{~mL}, 62$ mol\%) and propargylic alcohol Z-18-1 (1.5 g, $7.2 \mathrm{mmol})$ were then added. The nitrogen atmosphere was exchanged to $\mathrm{H}_{2}$-atmosphere ( 1 bar, balloon). The reaction mixture was stirred at room temperature until it was completed as determined by TLC analysis. The reaction mixture was filtered through celite and washed with EtOAc $(50 \mathrm{~mL})$. The combined organic layers was concentrated in vacuum, and the residue was purified by flash column chromatography on silica gel to afford Z-18-2 in $60 \%$ yield (0.9 g) as a white solid. 
Step 3: To a solution of Z-18-2 (0.9 g, $4.3 \mathrm{mmol})$ in DCM (20 mL) was added DessMartin periodinane $(2.2 \mathrm{~g}, 5.2 \mathrm{mmol})$. The reaction mixture was stirred at room temperature for 1 hour, and the solvent was removed under reduced pressure. The residue was purified by flash column chromatography on silica gel to give aldehyde $\mathbf{Z}$ 18-3 in a quantitative yield.

Step 4: Diene Z-18 (583 mg, 69\% yield) was prepared from aldehyde Z-18-3 (850 mg, $4.1 \mathrm{mmol}$ ) according to the step 5 in General Procedure $\mathrm{B}$, but the reaction was performed at $0{ }^{\circ} \mathrm{C}$ for 4 hours. White solid, mp: $52-54^{\circ} \mathrm{C}$.

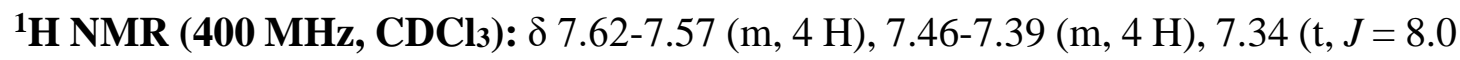
Hz, $1 \mathrm{H}), 6.95$ (ddd, $J=16.0,12.0,12.0 \mathrm{~Hz}, 1 \mathrm{H}), 6.49$ (d, $J=12.0 \mathrm{~Hz}, 1 \mathrm{H}), 6.29$ (dd, $J=12.0,12.0 \mathrm{~Hz}, 1 \mathrm{H}), 5.40(\mathrm{~d}, J=16.0 \mathrm{~Hz}, 1 \mathrm{H}), 5.25(\mathrm{~d}, J=12.0 \mathrm{~Hz}, 1 \mathrm{H})$.

${ }^{13}$ C NMR (100 MHz, CDCl3): $\delta$ 140.7, 139.8, 136.4, 133.2, 130.9, 129.9, 129.4, 128.8, $127.3,127.0,126.9,119.8$.

IR (neat, cm$^{-1}$ ): 1485, 1441, 1403, 1131, 1077, 1005, 904, 852, 762, 691.

HRMS (EI): [M] ${ }^{+}$calcd for $\mathrm{C}_{16} \mathrm{H}_{14}$ 206.1096, found: 206.1092 . 


\subsection{Synthesis of Vinyl Triflates}<smiles>C1CCCCC1</smiles>

$2 \mathbf{a}$<smiles></smiles>

$\mathbf{2 b}$

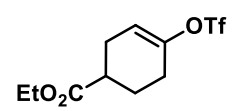

2c

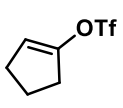

2d

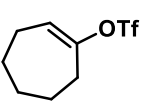

$2 e$

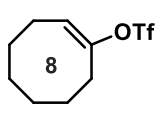

$2 f$

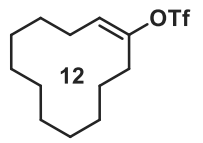

$2 \mathrm{~g}$<smiles>CCCOC1=CC(O)CCC1</smiles>

2h

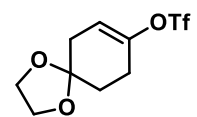

2j<smiles>[C+]C1CC=C([O])CC1</smiles>

2k

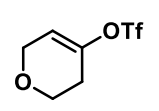

2I

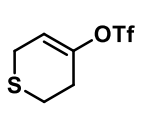

$2 m$<smiles>OC1=CCCc2ccccc21</smiles>

$2 n$<smiles></smiles>

20<smiles>[Z1]C(=O)OCCCCC(=C)OCC</smiles>

$2 p$<smiles>C=C(CCCCCO)OCC</smiles>

$2 \mathbf{u}$
$2 q$<smiles>CC(C)=C(C)O</smiles><smiles>C=C(O)CCCCC(=O)Nc1ccc(C)cc1</smiles><smiles>[O]C1=CCC(c2ccccc2)CC1</smiles><smiles>C=C(C)[C@H]1CC=C2C=C(O)CC(C)C2(C)C1</smiles>

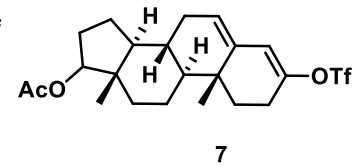

Vinyl triflates $2 \mathbf{a}^{17}, \mathbf{2} \mathbf{b}^{18}, \mathbf{2} \mathbf{c}^{19}, \mathbf{2} \mathbf{d}^{17}, \mathbf{2} \mathbf{e}^{17}, \mathbf{2} \mathbf{f}^{17}, \mathbf{2} \mathbf{g}^{20}, \mathbf{2} \mathbf{h}^{21}, \mathbf{2} \mathbf{i}^{21}, \mathbf{2} \mathbf{j}^{17}, \mathbf{2} \mathbf{k}^{17}, \mathbf{2} \mathbf{1}^{17}, \mathbf{2} \mathbf{m}^{22}$, $\mathbf{2 n}^{17}, \mathbf{2} \mathbf{o}^{21}, \mathbf{2} \mathbf{p}^{23}, \mathbf{2} \mathbf{q}^{24}, \mathbf{2} \mathbf{r}^{25}, \mathbf{2} \mathbf{t}^{24}, \mathbf{2} \mathbf{u}^{25}, \mathbf{2} \mathbf{v}^{24}, \mathbf{2} \mathbf{w},{ }^{24} \mathbf{5}^{26}, \mathbf{7}^{27}$ are known compounds and were synthesized according to the literature procedure. The preparation of new vinyl triflates, and their characterization data are provided as follows.

\section{6-(((Trifluoromethyl)sulfonyl)oxy)hept-6-en-1-yl 2-(2-fluoro-[1,1'-biphenyl]-4-} yl)propanoate (2s)<smiles>C=C(O)CCCCOC(=C)CCCCCOC(=O)C(C)(C)c1ccc(-c2ccccc2)c(F)c1</smiles>

To a solution of flurbiprofen $(1.1 \mathrm{~g}, 4.5 \mathrm{mmol})$ in DCM $(10.0 \mathrm{~mL})$ was added DCC (928 $\mathrm{mg}, 4.5 \mathrm{mmol})$ and DMAP $(36.7 \mathrm{mg}, 0.3 \mathrm{mmol})$ at $0{ }^{\circ} \mathrm{C}$. The reaction mixture was stirred at $0{ }^{\circ} \mathrm{C}$ for $30 \mathrm{~min}$, and a solution of vinyl triflate $2 \mathbf{u}^{23}(786 \mathrm{mg}, 3.0 \mathrm{mmol})$ in DCM $(5.0 \mathrm{~mL})$ was added. The reaction mixture was stirred at room temperature for overnight. The reaction was quenched with saturated aqueous solution of $\mathrm{NaHCO}_{3}$ and extracted with DCM $(3 \times 20.0 \mathrm{~mL})$. The combined organic layers was washed with brine, dried over anhydrous $\mathrm{MgSO}_{4}$, and concentrated under reduced pressure. The residue was purified by flash chromatography on silica gel to give the product $2 \mathrm{~s}$ in $73 \%$ 
yield (1.07 g) as a colorless oil with a few inseparable impurity.

${ }^{1} \mathbf{H}$ NMR (600 MHz, CDCl3): $\delta 7.52$ (d, $\left.J=7.8 \mathrm{~Hz}, 2 \mathrm{H}\right), 7.43-7.33(\mathrm{~m}, 4 \mathrm{H}), 7.15-$ $7.11(\mathrm{~m}, 2 \mathrm{H}), 5.06(\mathrm{~d}, J=3.6 \mathrm{~Hz}, 1 \mathrm{H}), 4.86(\mathrm{~d}, J=3.6 \mathrm{~Hz}, 1 \mathrm{H}), 4.10-4.08(\mathrm{~m}, 2 \mathrm{H})$, $3.74(\mathrm{q}, J=7.2 \mathrm{~Hz}, 1 \mathrm{H}), 2.29$ (t, $J=7.8 \mathrm{~Hz}, 2 \mathrm{H}), 1.64-1.60$ (m, $2 \mathrm{H}), 1.58-1.49$ (m, 5 H), $1.35-1.30(\mathrm{~m}, 2 \mathrm{H})$.

${ }^{13}$ C NMR (150 MHz, CDCl3): $\delta$ 173.9, 159.6 (d, $\left.J=248 \mathrm{~Hz}\right), 156.5,141.9$ (d, $J=7.5$ $\mathrm{Hz}), 135.4,130.7$ (d, $J=4.5 \mathrm{~Hz}), 128.8(\mathrm{~d}, J=3.0 \mathrm{~Hz}), 128.4,127.7(\mathrm{~d}, J=13.5 \mathrm{~Hz})$, 127.6, $123.5(\mathrm{~d}, J=3.0 \mathrm{~Hz}), 118.4(\mathrm{~d}, J=318 \mathrm{~Hz}), 115.2(\mathrm{~d}, J=24 \mathrm{~Hz}), 104.2,64.5$, $45.0,33.6,28.1,25.4,24.8,18.2$.

${ }^{19}$ F NMR (376 MHz, CDCl3): $\delta$-74.2, -117.7.

IR (neat, cm$^{-1}$ ): 2943, 2872, 1735, 1672, 1485, 1418, 1213, 1148, 928, 904, 768, 699.

HRMS (ESI): $[\mathrm{M}+\mathrm{Na}]^{+}$calcd for $\mathrm{C}_{23} \mathrm{H}_{24} \mathrm{~F}_{4} \mathrm{NaO}_{5} \mathrm{~S} 511.1173$, found: 511.1186 . 


\section{Regiocontrolled Reductive Vinylation of Aliphatic 1,3-Dienes with Vinyl Triflates by Nickel Catalysis}

\subsection{Optimization of Reaction Parameters}

General Procedure C. The procedure was conducted in an argon-filled glove box. To a reaction tube equipped with a magnetic stir bar was charged with catalyst (5 mol \%, $0.005 \mathrm{mmol})$, ligand $(6 \mathrm{~mol} \%, 0.006 \mathrm{mmol})$, bases (2.0 equiv., $0.2 \mathrm{mmol})$ and solvent (0.5 mL). The hydride sources (2.5 equiv., $0.25 \mathrm{mmol})$, diene $1 \mathbf{a}(15.0 \mathrm{mg}, 0.1 \mathrm{mmol})$ and vinyl triflates $\mathbf{2 a}(41.4 \mathrm{mg}, 0.18 \mathrm{mmol})$ were added in sequence. The reaction tube was sealed and removed from the glove box. The reaction mixture was stirred at $0{ }^{\circ} \mathrm{C}$ for 24 hours. It was diluted with ethyl acetate $(10 \mathrm{~mL})$, washed with water, brine, and dried over anhydrous $\mathrm{Na}_{2} \mathrm{SO}_{4}$. A $0.2 \mathrm{~mL}$ of solution was collected and diluted with ethyl acetate $(2 \mathrm{~mL})$. The yield and the ratio of $\mathbf{3 a} / \mathbf{4} \mathbf{a}$ were determined by $\mathrm{GC}$ analysis versus the internal standard (Dodecane).

\subsection{Ni-catalyzed Hydrovinylation of 1,3-Diene 1 and Vinyl Triflates 2}

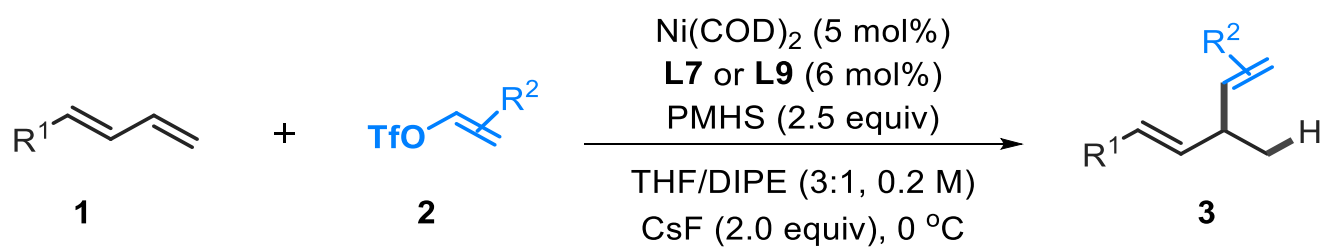

General Procedure D. The procedure was conducted in an argon-filled glove box. To a reaction tube equipped with a magnetic stir bar was charged with $\mathrm{Ni}(\mathrm{COD})_{2}(2.8 \mathrm{mg}$, $0.01 \mathrm{mmol}$ ), $\mathbf{L} 7$ (for aromatic dienes, $3.4 \mathrm{mg}, 0.012 \mathrm{mmol}$ ) or $\mathbf{L 9}$ (for aliphatic dienes, $6.1 \mathrm{mg}, 0.012 \mathrm{mmol}), \mathrm{CsF}(60.8 \mathrm{mg}, 0.4 \mathrm{mmol})$, and THF/DIPE (3:1, $1 \mathrm{~mL}, 0.2 \mathrm{M})$. PMHS (111.3 mg, $0.5 \mathrm{mmol})$, dienes $1(0.2 \mathrm{mmol})$ and vinyl triflates $2(0.36 \mathrm{mmol})$ were then added in sequence. The reaction tube was sealed and removed from the glove box. The reaction mixture was stirred at $0^{\circ} \mathrm{C}$ for $48 \mathrm{~h}$. The solvent was removed, and the residue was purified by flash chromatography on silica gel to afford the desired product 3 . 


\subsection{Characterization Data of Products}

\section{(E)-1-(4,8-dimethylnona-3,7-dien-2-yl)cyclohex-1-ene (3a)}<smiles>CC(C)=CCC/C(C)=C/C(C)C1=CCCCC1</smiles>

The target compound was prepared according to the General Procedure D from the reaction of $1 \mathbf{a}(30.0 \mathrm{mg}, 0.2$ mmol) and 2a (82.8 mg, $0.36 \mathrm{mmol})$.

$43.6 \mathrm{mg}, 94 \%$ isolated yield, the regioisomeric ratio of up to $86: 1$, colorless oil.

${ }^{1}$ H NMR (400 MHz, CDCl 3 ): $\delta$ 5.42-5.40 (m, 1 H), 5.08 (tt, $J=8.0,1.2$ Hz, 1 H), 4.98 $(\mathrm{dd}, J=8.0,1.2 \mathrm{~Hz}, 1 \mathrm{H}), 2.91-2.83(\mathrm{~m}, 1 \mathrm{H}), 2.08(\mathrm{q}, J=7.2 \mathrm{~Hz}, 2 \mathrm{H}), 2.00-1.83(\mathrm{~m}$, $6 \mathrm{H}), 1.68(\mathrm{~d}, J=0.8 \mathrm{~Hz}, 3 \mathrm{H}), 1.63-1.51(\mathrm{~m}, 10 \mathrm{H}), 1.02(\mathrm{~d}, J=6.8 \mathrm{~Hz}, 3 \mathrm{H})$.

${ }^{13}$ C NMR (100 MHz, CDCl3): $\delta$ 142.0, 133.7, 131.2, 129.7, 124.4, 118.8, 39.8, 39.4, $26.7,26.6,25.7,25.3,23.2,22.8,19.5,17.7,16.1$.

IR (neat, $\left.\mathbf{c m}^{-1}\right):$ 2925, 2856, 1666, 1449, 1379, 1014, 919, 835, 802, 734.

HRMS (EI): [M] ${ }^{+}$calcd for $\mathrm{C}_{17} \mathrm{H}_{28}$ 232.2191, found: 232.2189 .

\section{(E)-4-(hept-3-en-2-yl)-1-tosyl-1,2,3,6-tetrahydropyridine (3b)}

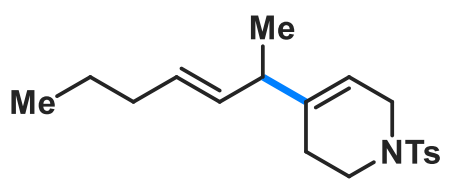

The target compound was prepared according to the General Procedure D from the reaction of $\mathbf{1 b}(19.2 \mathrm{mg}, 0.2$ $\mathrm{mmol})$ and $\mathbf{2 b}(138.6 \mathrm{mg}, 0.36 \mathrm{mmol})$.

$42.0 \mathrm{mg}, 63 \%$ yield, the regioisomeric ratio of $>20: 1$, colorless oil.

${ }^{1} \mathbf{H}$ NMR (400 MHz, CDCl3): $\delta 7.67(\mathrm{~d}, J=8.0 \mathrm{~Hz}, 2 \mathrm{H}), 7.31(\mathrm{~d}, J=8.0 \mathrm{~Hz}, 2 \mathrm{H})$, 5.38-5.31 (m, $2 \mathrm{H}), 5.19$ (ddt, $J=16.0,8.0,1.2 \mathrm{~Hz}, 1 \mathrm{H}), 3.57(\mathrm{~d}, J=2.4 \mathrm{~Hz}, 2 \mathrm{H})$, 3.19-3.10 (m, 2 H), 2.68-2.61 (m, 1 H), 2.42 (s, 3 H), 2.14-2.05 (m, 2 H), 1.93 (q, $J=$ $8.0 \mathrm{~Hz}, 2 \mathrm{H}), 1.39-1.28(\mathrm{~m}, 2 \mathrm{H}), 1.02(\mathrm{~d}, J=6.8 \mathrm{~Hz}, 3 \mathrm{H}), 0.86(\mathrm{t}, J=8.0 \mathrm{~Hz}, 3 \mathrm{H})$.

${ }^{13}$ C NMR (100 MHz, CDCl3): $\delta$ 143.4, 140.2, 133.3, 133.2, 130.1, 129.5, 127.7, 115.2, $44.9,43.1,42.9,34.5,26.5,22.6,21.5,18.3,13.6$.

IR (neat, cm$^{-1}$ ): 2961, 2920, 2370, 1699, 1463, 1351, 1164, 1096, 945, 816, 734, 669. HRMS (ESI): [M+Na] ${ }^{+}$calcd for $\mathrm{C}_{19} \mathrm{H}_{27} \mathrm{NNaO}_{2} \mathrm{~S} 356.1655$, found: 356.1654 . 


\section{(E)-1-(undec-3-en-2-yl)cyclohex-1-ene (3c)}<smiles>CCCCCCCC=CC(C)C1=CCCCC1</smiles>

The target compound was prepared according to the General Procedure D from the reaction of $1 \mathrm{c}(30.4 \mathrm{mg}, 0.2 \mathrm{mmol})$ and 2a (82.8 mg, $0.36 \mathrm{mmol})$.

$38.4 \mathrm{mg}, 82 \%$ yield, the regioisomeric ratio of $>20: 1$, colorless oil.

${ }^{1}$ H NMR (400 MHz, CDCl $)$ ) $\delta$ 5.43-5.41 (m, 1 H), 5.39-5.29 (m, 2 H), 2.66-2.59 (m, $1 \mathrm{H}), 2.05-1.84(\mathrm{~m}, 6 \mathrm{H}), 1.62-1.51(\mathrm{~m}, 4 \mathrm{H}), 1.38-1.27(\mathrm{~m}, 10 \mathrm{H}), 1.06(\mathrm{~d}, J=7.2 \mathrm{~Hz}$, $3 \mathrm{H}), 0.88(\mathrm{t}, J=8.0 \mathrm{~Hz}, 3 \mathrm{H})$.

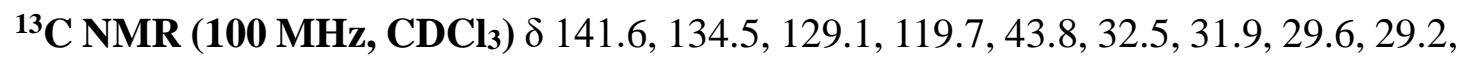
$29.1,26.4,25.3,23.1,22.74,22.66,18.8,14.1$.

IR (neat, $\mathbf{c m}^{-1}$ ): 2955, 2926, 2855, 1657, 1456, 1089, 968, 749.

HRMS (EI): [M] ${ }^{+}$calcd for $\mathrm{C}_{17} \mathrm{H}_{30}$ 234.2348, found: 234.2347.

\section{Ethyl 4-((E)-4-cyclohexylbut-3-en-2-yl)cyclohex-3-ene-1-carboxylate (3d)}

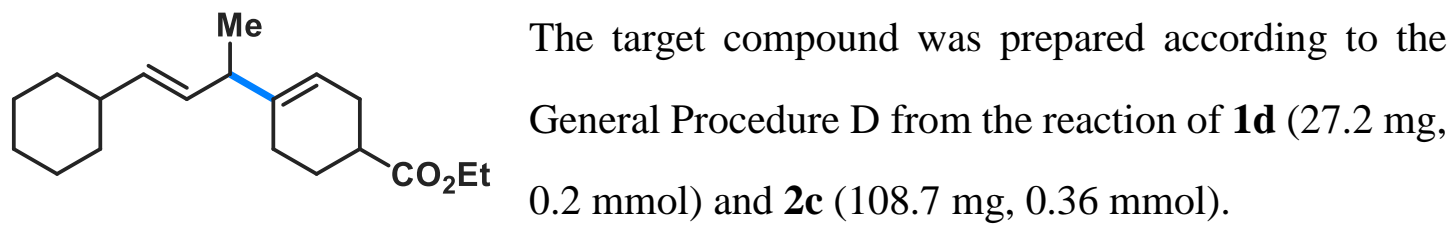

$52.2 \mathrm{mg}, 90 \%$ yield, the regioisomeric ratio of $>20: 1, \mathrm{dr}=2: 1$, colorless oil.

${ }^{1} \mathbf{H}$ NMR (400 MHz, CDCl3): $\delta 5.41$ (s, 1 H), 5.35-5.21 (m, 2 H), 4.13 (q, $J=8.0 \mathrm{~Hz}$, $2 \mathrm{H}), 2.68-2.59(\mathrm{~m}, 1 \mathrm{H}), 2.51-2.43(\mathrm{~m}, 1 \mathrm{H}), 2.25-2.23(\mathrm{~m}, 2 \mathrm{H}), 2.07-1.88(\mathrm{~m}, 4 \mathrm{H})$, 1.73-1.60 (m, $6 \mathrm{H}), 1.31-1.20$ (m, $5 \mathrm{H}), 1.18-1.12$ (m, $1 \mathrm{H}), 1.10-1.00$ (m, $5 \mathrm{H})$.

${ }^{13}$ C NMR (100 MHz, CDCl3): $\delta$ [176.1, 175.9], [141.3, 141.2], [135.5, 135.3], [131.5, 131.4], [117.9, 117.8], 60.2, [43.4, 43.2], 40.6, 39.6, 33.3, 27.7, 26.2, 26.1, 25.72, 25.67, [18.9, 18.5], 14.2.

IR (neat, cm$^{-1}$ ): 2925, 2851, 1735, 1449, 1375, 1307, 1254, 1225, 1174, 1033, 968.

HRMS (ESI): $[\mathrm{M}+\mathrm{H}]{ }^{+}$calcd for $\mathrm{C}_{19} \mathrm{H}_{31} \mathrm{O}_{2} 291.2319$, found: 291.2317. 


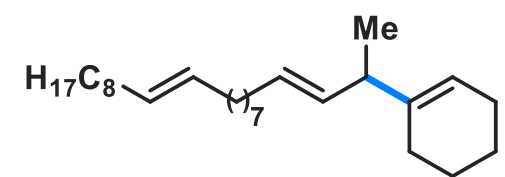

The target compound was prepared according to the General Procedure D from the reaction of 1 e $(58.1 \mathrm{mg}$, $0.2 \mathrm{mmol})$ and $\mathbf{2 a}(82.8 \mathrm{mg}, 0.36 \mathrm{mmol})$.

$49.2 \mathrm{mg}, 66 \%$ yield, the regioisomeric ratio of $>20: 1$, colorless oil.

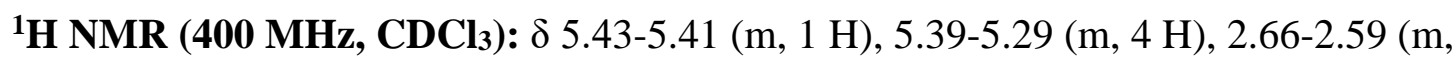
$1 \mathrm{H}), 2.04-1.88(\mathrm{~m}, 10 \mathrm{H}), 1.62-1.51(\mathrm{~m}, 4 \mathrm{H}), 1.33-1.27(\mathrm{~m}, 22 \mathrm{H}), 1.06(\mathrm{~d}, J=7.2 \mathrm{~Hz}$, $3 \mathrm{H}), 0.88(\mathrm{t}, J=8.0 \mathrm{~Hz}, 3 \mathrm{H})$.

${ }^{13}$ C NMR (100 MHz, CDCl $)$ ): $\delta$ 141.6, 134.5, 129.91, 129.87, 129.0, 119.7, 43.8, 32.5, 31.9, 29.78, 29.76, 29.6, 29.5, 29.4, 29.32 (2 C), 29.29, 29.1, 27.2 (2 C), 26.4, 25.3, $23.1,22.8,22.7,18.8,14.1$.

IR (neat, $\mathbf{c m}^{-1}$ ): 2925, 2854, 1657, 1459, 1374, 1012, 967, 722.

GC-MS (EI): $[\mathrm{M}]^{+}$calcd for $\mathrm{C}_{27} \mathrm{H}_{48} 372.38$, found: 372.39 .

(E)-1-(6,10-dimethylundeca-3,9-dien-2-yl)cyclohex-1-ene (3f)<smiles>CC(C)=CCC(C)CC=CC(C)C1=CCCCC1</smiles>

The target compound was prepared according to the General Procedure D from the reaction of $\mathbf{1 f}(35.6 \mathrm{mg}$, $0.2 \mathrm{mmol})$ and $\mathbf{2 a}(82.8 \mathrm{mg}, 0.36 \mathrm{mmol})$.

$48.4 \mathrm{mg}, 93 \%$ yield, the regioisomeric ratio of $>20: 1, \mathrm{dr}=1.3: 1$, colorless oil.

${ }^{1} \mathbf{H}$ NMR (600 MHz, CDCl $)$ : $\delta 5.42$ (s, $\left.1 \mathrm{H}\right), 5.37-5.29$ (m, $\left.2 \mathrm{H}\right), 5.09$ (t, $J=7.2 \mathrm{~Hz}$, $1 \mathrm{H}), 2.66-2.62$ (m, $1 \mathrm{H}), 2.02-1.97$ (m, $4 \mathrm{H}), 1.96-1.92$ (m, $2 \mathrm{H}), 1.88-1.80$ (m, $2 \mathrm{H})$, 1.68 (s, $3 \mathrm{H}), 1.60-1.56(\mathrm{~m}, 5 \mathrm{H}), 1.55-1.52$ (m, $2 \mathrm{H}), 1.49-1.43$ (m, $1 \mathrm{H}), 1.36-1.30(\mathrm{~m}$, $1 \mathrm{H}), 1.16-1.10(\mathrm{~m}, 1 \mathrm{H}), 1.06(\mathrm{~d}, J=6.6 \mathrm{~Hz}, 3 \mathrm{H}), 0.86(\mathrm{~d}, J=6.6 \mathrm{~Hz}, 3 \mathrm{H})$.

${ }^{13}$ C NMR (150 MHz, CDCl3): $\delta$ 141.5, 135.9, 131.0, 127.4, 125.0, [119.8, 119.7], 43.9, 39.9, [36.64, 36.63], 32.9, [26.52, 26.47], 25.7, 25.6, 25.3, 23.1, 22.8, [19.46, 19.45], 18.8, 17.6.

IR (neat, cm$^{-1}$ ): 2926, 2857, 1659, 1452, 1376, 1081, 969, 918, 801.

HRMS (EI): [M] ${ }^{+}$calcd for $\mathrm{C}_{19} \mathrm{H}_{32} 260.2504$, found: 260.2494 . 
tert-butyl (E)-(8-(cyclohex-1-en-1-yl)non-6-en-1-yl)carbamate (3g)

Boc

The target compound was prepared according to the General Procedure D from the reaction of $\mathbf{1 g}(47.8 \mathrm{mg}, 0.2 \mathrm{mmol})$ and $\mathbf{2 a}(82.8 \mathrm{mg}, 0.36 \mathrm{mmol})$.

$39.8 \mathrm{mg}, 62 \%$ yield, the regioisomeric ratio of $>20: 1$, colorless oil.

${ }^{1}$ H NMR (400 MHz, CDCl3): $\delta$ 5.42-5.29 (m, 3 H), 4.50 (s, $\left.1 \mathrm{H}\right), 3.11-3.08$ (m, $2 \mathrm{H}$ ), 2.65-2.59 (m, 1 H), 1.99-1.96 (m, 4 H), 1.93-1.84 (m, 2 H), 1.61-1.52 (m, 4 H), 1.47$1.44(\mathrm{~m}, 11 \mathrm{H}), 1.39-1.28(\mathrm{~m}, 4 \mathrm{H}), 1.05(\mathrm{~d}, J=6.8 \mathrm{~Hz}, 3 \mathrm{H})$

${ }^{13}$ C NMR (100 MHz, CDCl $)$ ): $\delta$ 156.0, 141.5, 134.8, 128.6, 119.8, 79.0, 43.8, 40.6, $32.4,29.9,29.2,28.4,26.4,26.3,25.3,23.1,22.7,18.8$

IR (neat, cm$^{-1}$ ): 2928, 2860, 16910, 1522, 1452, 1365, 1273, 1252, 1170, 968, 868.

HRMS (ESI): $[\mathrm{M}+\mathrm{Na}]^{+}$calcd for $\mathrm{C}_{20} \mathrm{H}_{35} \mathrm{NNaO}_{2} 344.2560$, found: 344.2557 .

\section{(E)-8-(cyclohex-1-en-1-yl)non-6-en-1-ol (3h)}<smiles>CC(C=CO)C1=CCCCC1</smiles>

The target compound was prepared according to the General Procedure D from the reaction of $\mathbf{1 h}(28.0 \mathrm{mg}, 0.2 \mathrm{mmol})$ and 2a (82.8 mg, $0.36 \mathrm{mmol})$. After $48 \mathrm{~h}, \mathrm{TBAF}(52.3 \mathrm{mg}, 0.2 \mathrm{mmol})$ was added and the reaction was stirred at room temperature for additional $30 \mathrm{~min}$. The solvent was removed, and the residue was purified by flash chromatography on silica gel to afford the desired product.

$23.6 \mathrm{mg}, 53 \%$ yield, the regioisomeric ratio of $>20: 1$, colorless oil.

${ }^{1}$ H NMR (400 MHz, CDCl3): $\delta$ 5.43-5.41 (m, 1 H), 5.39-5.30 (m, $\left.2 \mathrm{H}\right), 3.64(\mathrm{t}, J=6.8$ Hz, 2 H), 2.66-2.58 (m, 1 H), 2.05-1.98 (m, 4 H), 1.94-1.82 (m, 2 H), 1.65-1.51 (m, 6 H), 1.42-1.31 (m, $5 \mathrm{H}), 1.06(\mathrm{~d}, J=7.2 \mathrm{~Hz}, 3 \mathrm{H})$.

${ }^{13}$ C NMR (100 MHz, CDCl3): $\delta$ 141.5, 134.8, 128.7, 119.8, 63.0, 43.8, 32.6, 32.4, 29.3, $26.4,25.3,25.2,23.1,22.7,18.8$.

IR (neat, cm$^{-1}$ ): 3404, 2964, 2928, 2869, 1656, 1450, 1378, 1263, 1057.

HRMS (ESI): $[\mathrm{M}+\mathrm{H}]^{+}$calcd for $\mathrm{C}_{15} \mathrm{H}_{27} \mathrm{O}$ 223.2056, found: 223.2056. 


\section{(E)-8-(1-tosyl-1,2,3,6-tetrahydropyridin-4-yl)non-6-en-1-yl (tert-butoxycarbonyl)-}

\section{$L$-alaninate (3i)}<smiles>CC(C)NC(=O)OCC=CC(C)C1=CC[N+](=S)CC1</smiles>

The target compound was prepared according to the

General Procedure D from the reaction of $1 \mathbf{i}(62.2 \mathrm{mg}$, $0.2 \mathrm{mmol})$ and $\mathbf{2 b}(138.6 \mathrm{mg}, 0.36 \mathrm{mmol})$.

$66.9 \mathrm{mg}, 61 \%$ yield, the regioisomeric ratio of $>20: 1, \mathrm{dr}=1: 1$ (dr value was determined by HPLC), colorless oil.

${ }^{1}$ H NMR (400 MHz, CDCl3): $\delta 7.67(\mathrm{~d}, J=8.0 \mathrm{~Hz}, 2 \mathrm{H}), 7.31(\mathrm{~d}, J=8.0 \mathrm{~Hz}, 2 \mathrm{H})$, 5.38-5.31 (m, $2 \mathrm{H}), 5.21(\mathrm{dd}, J=16.0,8.0 \mathrm{~Hz}, 1 \mathrm{H}), 5.08(\mathrm{~s}, 1 \mathrm{H}), 4.29-4.27(\mathrm{~m}, 1 \mathrm{H})$, 4.16-4.07 (m, 2 H), 3.56 (s, 2 H), 3.17-3.11 (m, 2 H), 2.66-2.61 (m, 1 H), 2.42 (s, 3 H), 2.17-2.04 (m, 2 H), 2.02-1.94 (m, 2 H), 1.65-1.62 (m, 2 H), 1.45 (s, 9 H), 1.38 (d, $J=$ $7.2 \mathrm{~Hz}, 4 \mathrm{H}), 1.35-1.34$ (m, $3 \mathrm{H}), 1.02(\mathrm{~d}, J=7.2 \mathrm{~Hz}, 3 \mathrm{H})$.

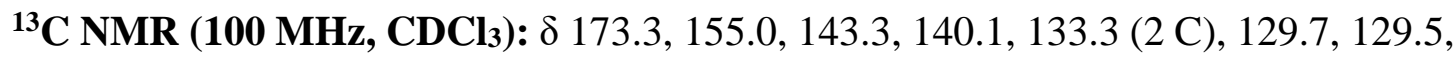
$127.7,115.3,79.7,65.2,49.2,44.9,43.0,42.8,32.2,29.0,28.29,28.26,26.5,25.2,21.4$, $18.7,18.3$.

IR (neat, $\mathbf{c m}^{-1}$ ): 2963, 2926, 1645, 1454, 1377, 1208, 1108, 1050, 904, 822.

HRMS (ESI): [M+Na] ${ }^{+}$calcd for $\mathrm{C}_{29} \mathrm{H}_{44} \mathrm{~N}_{2} \mathrm{NaO}_{6} \mathrm{~S}$ 571.2812, found: 571.2808.

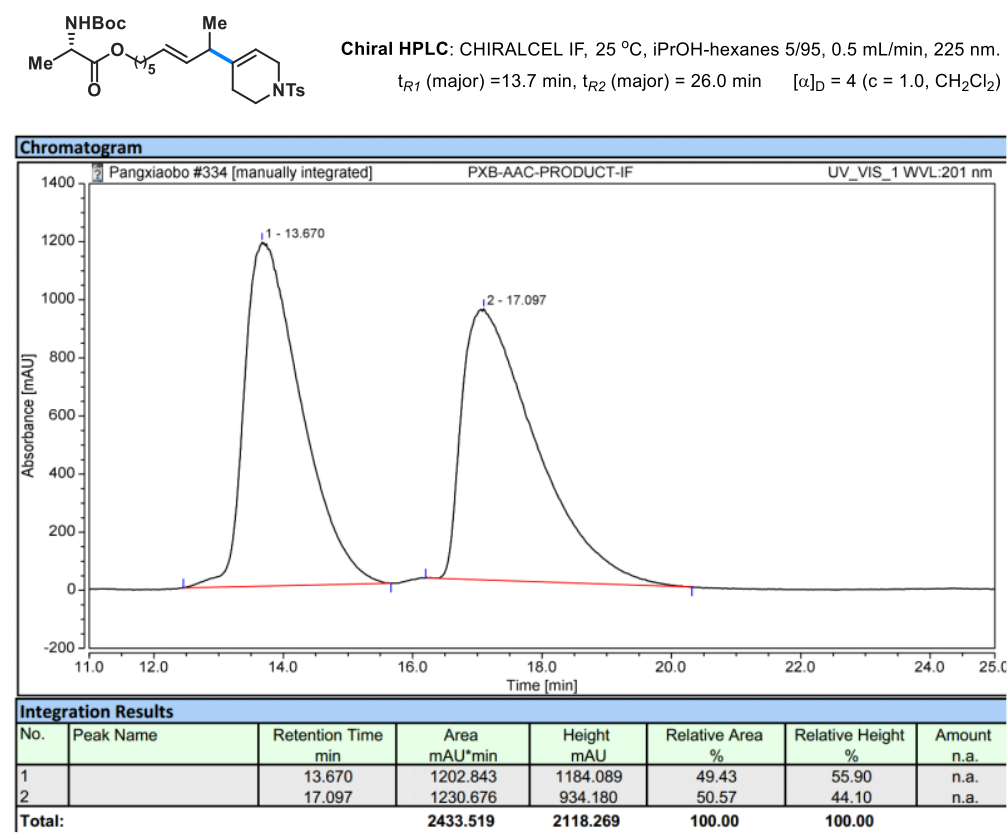


dimethylsilyl)oxy)-2-phenylacetate (3j)<smiles>CC(C=CCOC(=O)C([OH2+])c1ccccc1)C1=CC[S+](=O)(=S)CC1</smiles>

The target compound was prepared according to the General Procedure D from the reaction of $\mathbf{1 j}(77.6 \mathrm{mg}$, $0.2 \mathrm{mmol})$ and $\mathbf{2 b}(138.6 \mathrm{mg}, 0.36 \mathrm{mmol})$.

$102.5 \mathrm{mg}, 82 \%$ yield, the regioisomeric ratio of $>20: 1$, colorless oil.

${ }^{1} \mathbf{H}$ NMR (400 MHz, CDCl3): $\delta 7.67(\mathrm{~m}, J=8.0 \mathrm{~Hz}, 2 \mathrm{H}), 7.47(\mathrm{~d}, J=7.2 \mathrm{~Hz}, 2 \mathrm{H})$, 7.35-7.28 (m, 5 H), 5.35-5.28 (m, 2 H), 5.21-5.16 (m, 2 H), 4.09-4.03 (m, 2 H), 3.56 (d, $J=4.0 \mathrm{~Hz}, 2 \mathrm{H}), 3.19-3.10$ (m, $2 \mathrm{H}), 2.67-2.57$ (m, $1 \mathrm{H}), 2.41$ (s, $3 \mathrm{H}), 2.16-2.04$ (m, 2 H), $1.89(\mathrm{q}, J=8.0 \mathrm{~Hz}, 2 \mathrm{H}), 1.59-1.53(\mathrm{~m}, 2 \mathrm{H}), 1.31-1.20(\mathrm{~m}, 4 \mathrm{H}), 1.02(\mathrm{~d}, J=6.8$ Hz, 3 H), 0.92 (s, 9 H), 0.11 (s, 3 H), 0.03 (s, 3 H).

${ }^{13}$ C NMR (100 MHz, CDCl $)$ ): $\delta$ 172.1, 143.4, 140.1, 139.3, 133.3, 133.2, 129.8, 129.5, 128.2, 128.0, 127.7, 126.3, 115.3, 74.4, 64.9, 44.9, 43.0, 42.8, 32.3, 28.9, 28.3, 26.5, $25.7,25.2,21.4,18.3(2 \mathrm{C}),-5.1,-5.2$.

IR (neat, cm$^{-1}$ ): 2955, 2930, 2857, 1753, 1639, 1460, 1349, 1254, 1167, 943, 866, 729. HRMS (ESI): $[\mathrm{M}+\mathrm{Na}]^{+}$calcd for $\mathrm{C}_{35} \mathrm{H}_{51} \mathrm{NNaO}_{5} \mathrm{SSi}$ 648.3149, found: 648.3150.

(E)-8-(1-tosyl-1,2,3,6-tetrahydropyridin-4-yl)non-6-en-1-yl ferrocenecarboxylacetate (3k)

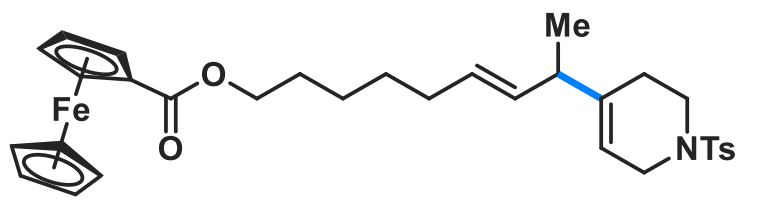

The target compound was prepared according to the General Procedure D from the reaction of $\mathbf{1 k}(70.4 \mathrm{mg}, 0.2$ mmol) and $2 \mathbf{b}(138.6 \mathrm{mg}, 0.36 \mathrm{mmol})$.

$47.1 \mathrm{mg}, 40 \%$ yield, the regioisomeric ratio of $>20: 1$, orange-red oil.

${ }^{1}$ H NMR (400 MHz, CDCl3): $\delta 7.66(\mathrm{~d}, J=8.0 \mathrm{~Hz}, 2 \mathrm{H}), 7.31(\mathrm{~d}, J=8.0 \mathrm{~Hz}, 2 \mathrm{H})$, 5.41-5.30 (m, $2 \mathrm{H}), 5.22(\mathrm{dd}, J=16.0,7.2 \mathrm{~Hz}, 1 \mathrm{H}), 4.80(\mathrm{t}, J=1.6 \mathrm{~Hz}, 2 \mathrm{H}), 4.39(\mathrm{t}, J$ $=1.6 \mathrm{~Hz}, 2 \mathrm{H}), 4.21-4.18(\mathrm{~m}, 7 \mathrm{H}), 3.56-3.54(\mathrm{~m}, 2 \mathrm{H}), 3.16-3.10$ (m, $2 \mathrm{H}), 2.68-2.59$ $(\mathrm{m}, 1 \mathrm{H}), 2.42(\mathrm{~s}, 3 \mathrm{H}), 2.17-2.09(\mathrm{~m}, 2 \mathrm{H}), 2.00(\mathrm{~d}, J=9.0 \mathrm{~Hz}, 2 \mathrm{H}), 1.73-1.68(\mathrm{~m}, 2$ H), $1.43-1.37$ (m, $4 \mathrm{H}), 1.02(\mathrm{~d}, J=6.8 \mathrm{~Hz}, 3 \mathrm{H})$. 
${ }^{13}$ C NMR (100 MHz, CDCl3): $\delta$ 171.7, 143.4, 140.1, 133.3, 133.2, 129.8, 129.5, 127.7, 115.3, 71.4, 71.2, 70.1, 69.7, 64.1, 44.9, 43.0, 42.8, 32.4, 29.1, 28.7, 26.5, 25.6, 21.5, 18.3.

IR (neat, $\mathbf{c m}^{-1}$ ): 2928, 2855, 1710, 1460, 1347, 1276, 1166, 1140, 945, 818, 775, 733, $675,549$.

HRMS (ESI): [M+Na ${ }^{+}$calcd for $\mathrm{C}_{32} \mathrm{H}_{39} \mathrm{FeNNaO}_{4} \mathrm{~S}$ 612.1841, found: 612.1844.

(R)-4-(but-3-en-2-yl)-1-tosyl-1,2,3,6-tetrahydropyridine (31)

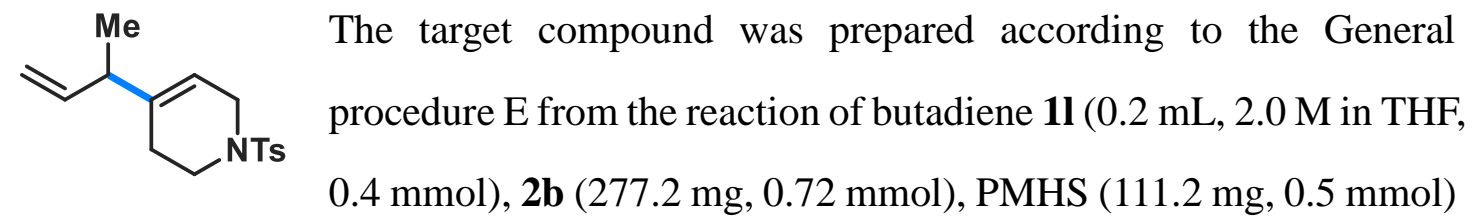
and CsF (60.8 mg, $0.4 \mathrm{mmol})$ in THF/DIPE $(1 \mathrm{~mL}, 0.4 \mathrm{M}, 3: 1)$ at $0{ }^{\circ} \mathrm{C}$ for 48 hours. $78.0 \mathrm{mg}, 67 \%$ yield, the regioisomeric ratio of 1,2/1,4-adducts is $9: 1$, the branched selectivity of 1,2-adduct is $3: 1$, colorless oil.

${ }^{1}$ H NMR (400 MHz, CDCl3): $\delta 7.67(\mathrm{~d}=8.0 \mathrm{~Hz}, 2 \mathrm{H}), 7.31(\mathrm{~d}, J=8.0 \mathrm{~Hz}, 2 \mathrm{H}), 5.67$ 5.59 (m, $1 \mathrm{H})$, 5.37-5.36 (m, $1 \mathrm{H}), 4.98-4.91(\mathrm{~m}, 2 \mathrm{H}), 3.58-3.55(\mathrm{~m}, 2 \mathrm{H}), 3.17-3.13$ (m, 1 H), 3.04-2.98 (m, 1 H), 2.73-2.68 (m, 1 H), 2.43 (s, 3 H), 2.18-2.05 (m, 2 H), 1.05 $(\mathrm{d}, J=6.8 \mathrm{~Hz}, 3 \mathrm{H})$.

${ }^{13}$ C NMR (100 MHz, CDCl3): $\delta$ 143.4, 141.4, 139.4, 129.5, 127.7, 121.7, 115.9, 114.0, $44.9,43.8,43.0,26.3,21.5,17.6$.

IR (neat, $\mathbf{c m}^{-1}$ ): 2963, 2924, 2852, 1637, 1460, 1345, 1164, 1096, 945, 816, 712, 549.

HRMS (ESI): $[\mathrm{M}+\mathrm{Na}]^{+}$calcd for $\mathrm{C}_{16} \mathrm{H}_{21} \mathrm{NNaO}_{2} \mathrm{~S} 314.1185$, found: 314.1185 .

\section{4-(3-Methylbut-3-en-2-yl)-1-tosyl-1,2,3,6-tetrahydropyridine (3m)}

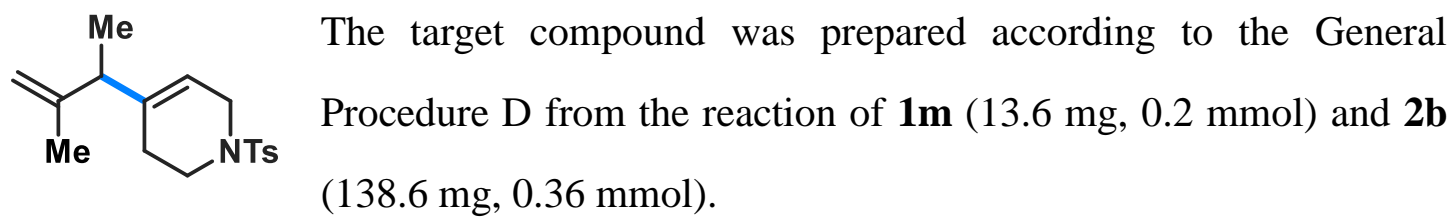

$31.1 \mathrm{mg}, 51 \%$ yield, the regioisomeric ratio of $>20: 1$, colorless oil.

${ }^{1}$ H NMR (400 MHz, CDCl3): $\delta 7.67(\mathrm{~d}, J=8.0 \mathrm{~Hz}, 2 \mathrm{H}), 7.31(\mathrm{~d}, J=8.0 \mathrm{~Hz}, 2 \mathrm{H})$, 
$5.39(\mathrm{~d}, J=0.8 \mathrm{~Hz}, 1 \mathrm{H}), 4.77(\mathrm{~s}, 1 \mathrm{H}), 4.70(\mathrm{~s}, 1 \mathrm{H}), 3.64-3.55(\mathrm{~m}, 2 \mathrm{H}), 3.21-3.15(\mathrm{~m}$, $1 \mathrm{H}), 3.12-3.06(\mathrm{~m}, 1 \mathrm{H}), 2.67(\mathrm{q}, J=8.0 \mathrm{~Hz}, 1 \mathrm{H}), 2.43(\mathrm{~s}, 3 \mathrm{H}), 2.13-2.09(\mathrm{~m}, 1 \mathrm{H})$, 2.00-1.95 (m, $1 \mathrm{H}), 1.53$ (s, $3 \mathrm{H}), 1.07$ (d, $J=7.2 \mathrm{~Hz}, 3 \mathrm{H})$.

${ }^{13}$ C NMR (100 MHz, CDCl3): $\delta$ 147.0, 143.4, 138.8, 133.4, 129.6, 127.7, 116.5, 110.8, 47.1, 44.9, 43.1, 26.3, 21.5, 20.3, 16.8 .

IR (neat, cm$^{-1}$ ): 2967, 2921, 2851, 1645, 1459, 1346, 1164, 1098, 944, 816, 719, 549. HRMS (ESI): $[\mathrm{M}+\mathrm{H}]^{+}$calcd for $\mathrm{C}_{17} \mathrm{H}_{24} \mathrm{NO}_{2} \mathrm{~S}$ 306.1522, found: 306.1526 .

\section{(E)-(3-(cyclohex-1-en-1-yl)but-1-en-1-yl)benzene (3n)}<smiles>CC(/C=C/c1ccccc1)C1=CCCCC1</smiles>

The target compound was prepared according to the General Procedure D from the reaction of $1 \mathbf{n}(26.0 \mathrm{mg}, 0.2 \mathrm{mmol})$ and 2a (82.8 $\mathrm{mg}, \quad 0.36 \mathrm{mmol}) ; 30.6 \mathrm{mg}, 72 \%$ yield, the regioisomeric ratio of $>20: 1$, colorless oil.

The use of complex A (2 mol\%) instead of $\mathrm{Ni}(\mathrm{COD})_{2}$ and $\mathbf{L 7}$ gave $3 \mathrm{n}$ in $70 \%$ yield (29.7 mg, > 20:1).

${ }^{1}$ H NMR (400 MHz, CDCl3): $\delta 7.35(\mathrm{~d}, J=8.0 \mathrm{~Hz}, 2 \mathrm{H}), 7.28(\mathrm{t}, J=8.0 \mathrm{~Hz}, 2 \mathrm{H})$, $7.18(\mathrm{tt}, J=8.0,1.2 \mathrm{~Hz}, 1 \mathrm{H}), 6.34(\mathrm{~d}, J=16.0 \mathrm{~Hz}, 1 \mathrm{H}), 6.16(\mathrm{dd}, J=16.0,8.0 \mathrm{~Hz}, 1$ H), $5.51(\mathrm{~d}, J=0.8 \mathrm{~Hz}, 1 \mathrm{H}), 2.89-2.82(\mathrm{~m}, 1 \mathrm{H}), 2.02-1.90(\mathrm{~m}, 4 \mathrm{H}), 1.64-1.52(\mathrm{~m}, 4$ H), $1.19(\mathrm{~d}, J=6.8 \mathrm{~Hz}, 3 \mathrm{H})$.

${ }^{13}$ C NMR (100 MHz, CDCl3): $\delta$ 140.9, 137.9, 135.2, 128.4, 128.3, 126.8, 126.0, 120.6, $44.2,26.6,25.3,23.1,22.7,18.5$.

IR (neat, cm$^{-1}$ ): 2932, 2859, 1651, 1451, 1268, 1072, 968, 748, 696.

HRMS (EI): [M] ${ }^{+}$calcd for $\mathrm{C}_{16} \mathrm{H}_{20} 212.1565$, found: 212.1564 .

\section{(E)-1-(3-(cyclohex-1-en-1-yl)but-1-en-1-yl)-4-methoxybenzene (3o)}<smiles>COc1ccc(/C=C/C(C)C2=CCCCC2)cc1</smiles>

The target compound was prepared according to the General Procedure D from the reaction of $10(32.0 \mathrm{mg}$, $0.2 \mathrm{mmol})$ and $\mathbf{2 a}(82.8 \mathrm{mg}, 0.36 \mathrm{mmol})$.

$44.1 \mathrm{mg}, 91 \%$ yield, the regioisomeric ratio of $>20: 1$, colorless oil. 
${ }^{1} \mathbf{H}$ NMR (400 MHz, CDCl $): \delta 7.29(\mathrm{~d}, J=8.0 \mathrm{~Hz}, 2 \mathrm{H}), 6.85-6.81(\mathrm{~m}, 2 \mathrm{H}), 6.29$ (d, $J=16.0 \mathrm{~Hz}, 1 \mathrm{H}), 6.02(\mathrm{dd}, J=16.0,8.0 \mathrm{~Hz}, 1 \mathrm{H}), 5.50(\mathrm{~d}, J=0.8 \mathrm{~Hz}, 1 \mathrm{H}), 3.80(\mathrm{~s}, 3$ H), 2.86-2.79 (m, $1 \mathrm{H}), 2.05-1.90(\mathrm{~m}, 4 \mathrm{H}), 1.64-1.52(\mathrm{~m}, 4 \mathrm{H}), 1.17$ (d, J = 7.2 Hz, 3 $\mathrm{H})$.

${ }^{13}$ C NMR (100 MHz, CDCl3): $\delta$ 158.6, 141.1, 133.0, 130.7, 127.6, 127.1, 120.4, 113.9, $55.3,44.2,26.5,25.3,23.1,22.7,18.6$.

IR (neat, cm$^{-1}$ ): 2930, 2835, 1606, 1582, 1510, 1458, 1249, 1174, 1034, 969, 915, 817. HRMS (EI): [M] ${ }^{+}$calcd for $\mathrm{C}_{17} \mathrm{H}_{22} \mathrm{O} 242.1671$, found: 242.1667 .

\section{(E)-4-(3-(cyclohex-1-en-1-yl)but-1-en-1-yl)phenol (3p)}<smiles>CC(/C=C/c1ccc(O)cc1)C1=CCCCC1</smiles>
The target compound was prepared according to the General Procedure D from the reaction of 1 p $(29.2 \mathrm{mg}, 0.2$ $\mathrm{mmol})$ and $2 \mathbf{a}(82.8 \mathrm{mg}, 0.36 \mathrm{mmol})$. After $48 \mathrm{~h}$, TBAF (52.3 $\mathrm{mg}, 0.2 \mathrm{mmol}$ ) was added and stirred at ambient temperature for additional 30 min. The solvent was removed, and the residue was purified by flash chromatography on silica gel to afford the desired product.

$29.7 \mathrm{mg}, 65 \%$ yield, the regioisomeric ratio of >20: 1 , colorless oil.

${ }^{1}$ H NMR (400 MHz, CDCl3): $\delta 7.24(\mathrm{~d}, J=8.0 \mathrm{~Hz}, 2 \mathrm{H}), 6.76(\mathrm{~d}, J=8.0 \mathrm{~Hz}, 2 \mathrm{H})$, $6.28(\mathrm{~d}, J=16.0 \mathrm{~Hz}, 1 \mathrm{H}), 6.01(\mathrm{dd}, J=16.0,8.0 \mathrm{~Hz}, 1 \mathrm{H}), 5.50(\mathrm{~s}, 1 \mathrm{H}), 4.75(\mathrm{br}, 1 \mathrm{H})$, 2.86-2.79 (m, 1 H), 2.01-1.86 (m, 4 H), 1.63-1.56 (m, 4 H), 1.17 (d, J= 7.2 Hz, 3 H). ${ }^{13}$ C NMR (100 MHz, CDCl3): $\delta$ 154.5, 141.1, 133.1, 130.9, 127.6, 127.3, 120.5, 115.3, $44.2,26.5,25.3,23.1,22.7,18.6$.

IR (neat, $\mathbf{c m}^{-1}$ ): 3389, 2927, 2856, 1610, 1512, 1447, 1237, 1170, 967, 816.

HRMS (ESI): $[\mathrm{M}+\mathrm{H}]{ }^{+}$calcd for $\mathrm{C}_{16} \mathrm{H}_{21} \mathrm{O} 229.1587$, found: 229.1589 .

\section{(E)-(4-(3-(cyclohex-1-en-1-yl)but-1-en-1-yl)phenyl)(methyl)sulfane (3q)}<smiles>CSc1ccc(/C=C/C(C)C2=CCCCC2)cc1</smiles>

The target compound was prepared according to the General Procedure D from the reaction of $\mathbf{1 q}(35.2 \mathrm{mg}$, $0.2 \mathrm{mmol})$ and $\mathbf{2 a}(82.8 \mathrm{mg}, 0.36 \mathrm{mmol})$. 
$44.9 \mathrm{mg}, 87 \%$ yield, the regioisomeric ratio of $>20: 1$, colorless oil.

${ }^{1}$ H NMR (400 MHz, CDCl3): $\delta 7.27(\mathrm{~d}, J=8.0 \mathrm{~Hz}, 2 \mathrm{H}), 7.19(\mathrm{~d}, J=8.0 \mathrm{~Hz}, 2 \mathrm{H})$, $6.29(\mathrm{~d}, J=16.0 \mathrm{~Hz}, 1 \mathrm{H}), 6.12(\mathrm{dd}, J=16.0,8.0 \mathrm{~Hz}, 1 \mathrm{H}), 5.50(\mathrm{~d}, J=1.2 \mathrm{~Hz}, 1 \mathrm{H})$, 2.88-2.81 (m, 1 H), 2.47 (s, 3 H), 2.02-1.89 (m, 4 H), 1.64-1.52 (m, 4 H), 1.18 (d, $J=$ $6.8 \mathrm{~Hz}, 3 \mathrm{H})$.

${ }^{13}$ C NMR (100 MHz, CDCl3): $\delta$ 140.9, 136.6, 135.0, 134.8, 127.7, 126.9, 126.5, 120.6, $44.2,26.6,25.3,23.1,22.7,18.5,16.1$.

IR (neat, $\mathbf{c m}^{-1}$ ): 2924, 2854, 1493, 1436, 1094, 1010, 966, 799.

HRMS (EI): $[\mathrm{M}]^{+}$calcd for $\mathrm{C}_{17} \mathrm{H}_{22} \mathrm{~S} 258.1422$, found: 258.1442 .

Methyl (E)-4-(3-(cyclohex-1-en-1-yl)but-1-en-1-yl)benzoate (3r)<smiles>CC(=O)c1ccc(/C=C/C(C)C2=CCCCC2)cc1</smiles>

The target compound was prepared according to the General Procedure D from the reaction of $1 \mathbf{r}(37.6 \mathrm{mg}$, $0.2 \mathrm{mmol})$ and $2 \mathbf{a}(82.8 \mathrm{mg}, 0.36 \mathrm{mmol})$.

$49.7 \mathrm{mg}, 92 \%$ yield, the regioisomeric ratio of $>20: 1$, colorless oil.

${ }^{1}$ H NMR (400 MHz, CDCl3): $\delta 7.88(\mathrm{~d}, J=8.0 \mathrm{~Hz}, 2 \mathrm{H}), 7.32(\mathrm{~d}, J=8.0 \mathrm{~Hz}, 2 \mathrm{H})$, $6.30(\mathrm{~d}, J=16.0 \mathrm{~Hz}, 1 \mathrm{H}), 6.22(\mathrm{dd}, J=16.0,8.0 \mathrm{~Hz}, 1 \mathrm{H}), 5.44(\mathrm{~d}, J=0.8 \mathrm{~Hz}, 1 \mathrm{H})$, $3.82(\mathrm{~s}, 3 \mathrm{H}), 2.84-2.77(\mathrm{~m}, 1 \mathrm{H}), 1.95-1.85(\mathrm{~m}, 4 \mathrm{H}), 1.56-1.47(\mathrm{~m}, 4 \mathrm{H}), 1.12(\mathrm{~d}, J=$ $6.8 \mathrm{~Hz}, 3 \mathrm{H})$.

${ }^{13}$ C NMR (100 MHz, CDCl3): $\delta$ 167.0, 142.4, 140.4, 138.1, 129.8, 128.3, 127.6, 125.9, $121.1,51.9,44.3,26.6,25.3,23.0,22.6,18.3$.

IR (neat, $\mathbf{c m}^{-1}$ ): 2927, 1723, 1608, 1566, 1433, 1277, 1179, 1109, 1017, 971, 766.

HRMS (ESI): $[\mathrm{M}+\mathrm{Na}]{ }^{+}$calcd for $\mathrm{C}_{18} \mathrm{H}_{22} \mathrm{NaO}_{2} 293.1512$, found: 293.1513.

\section{(E)-2-(3-(cyclohex-1-en-1-yl)but-1-en-1-yl)thiophene (3s)}<smiles>CC(/C=C/c1cccs1)C1=CCCCC1</smiles>

The target compound was prepared according to the General Procedure D from the reaction of $1 \mathrm{~s}(27.2 \mathrm{mg}, 0.2 \mathrm{mmol})$ and 2a (82.8 mg, $0.36 \mathrm{mmol})$.

$30.5 \mathrm{mg}, 70 \%$ yield, the regioisomeric ratio of $>20: 1$, colorless oil.

${ }^{1}$ H NMR (400 MHz, CDCl $)$ : $\delta 7.08(\mathrm{~d}, J=8.0 \mathrm{~Hz}, 1 \mathrm{H}), 6.94-6.92(\mathrm{~m}, 1 \mathrm{H}), 6.88(\mathrm{~d}$, 
$J=4.0 \mathrm{~Hz}, 1 \mathrm{H}), 6.47(\mathrm{~d}, J=16.0 \mathrm{~Hz}, 1 \mathrm{H}), 6.01(\mathrm{dd}, J=16.0,8.0 \mathrm{~Hz}, 1 \mathrm{H}), 5.50(\mathrm{~d}, J$ $=0.8 \mathrm{~Hz}, 1 \mathrm{H}), 2.85-2.79(\mathrm{~m}, 1 \mathrm{H}), 2.02-1.89(\mathrm{~m}, 4 \mathrm{H}), 1.64-1.52(\mathrm{~m}, 4 \mathrm{H}), 1.17(\mathrm{~d}, J=$ $7.2 \mathrm{~Hz}, 3 \mathrm{H})$.

${ }^{13}$ C NMR (100 MHz, CDCl3): $\delta$ 143.2, 140.6, 135.1, 127.2, 124.3, 123.1, 121.7, 120.9, $44.0,26.5,25.3,23.1,22.6,18.4$.

IR (neat, cm$^{-1}$ ): 2927, 2856, 1649, 1416, 1208, 1033, 956, 692.

HRMS (EI): [M] ${ }^{+}$calcd for $\mathrm{C}_{14} \mathrm{H}_{18} \mathrm{~S} 218.1129$, found: 218.1126 .

Ethyl 4-((E)-4-(furan-2-yl)but-3-en-2-yl)cyclohex-3-ene-1-carboxylate (3t)

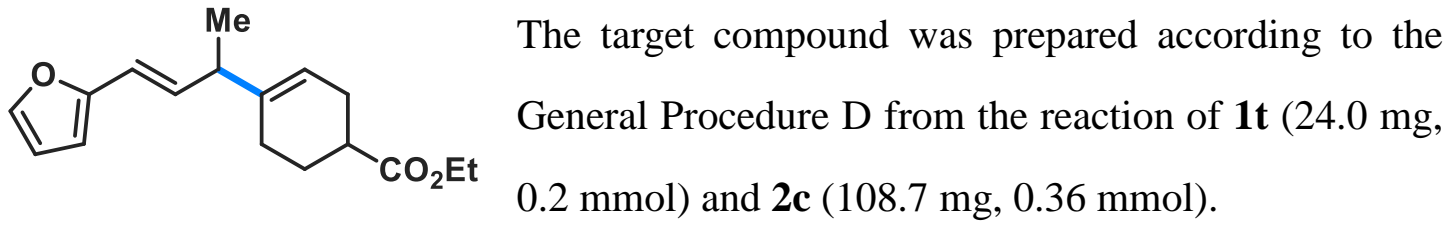

$41.1 \mathrm{mg}, 75 \%$ yield, the regioisomeric ratio of $>20: 1$, colorless oil.

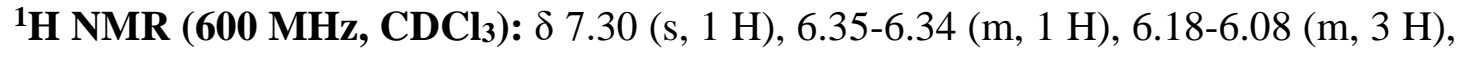
$5.50(\mathrm{~s}, 1 \mathrm{H}), 4.13$ (q, $J=6.0 \mathrm{~Hz}, 2 \mathrm{H}), 2.89-2.82$ (m, $1 \mathrm{H}), 2.56-2.47$ (m, $1 \mathrm{H}), 2.28$ $2.23(\mathrm{~m}, 2 \mathrm{H})$ 2.11-2.08 (m, $1 \mathrm{H}), 2.04-1.99(\mathrm{~m}, 2 \mathrm{H}), 1.71-1.63(\mathrm{~m}, 1 \mathrm{H}), 1.25(\mathrm{t}, J=$ $6.0 \mathrm{~Hz}, 3 \mathrm{H}), 1.17(\mathrm{~d}, J=7.2 \mathrm{~Hz}, 3 \mathrm{H})$.

${ }^{13}$ C NMR (100 MHz, CDCl3): $\delta$ 176.0, [153.18, 153.17], 141.3, [140.4, 140.2], [133.6, 133.5], [119.2, 119.0], [117.4, 117.3], 111.1, 106.4, 60.2, [43.5, 43.4], [39.53, 39.47], [27.72, 27.67], [25.8, 25.6], 18.6, 18.2, 14.2.

IR (neat, $\mathbf{c m}^{-1}$ ): 2965, 2933, 2163, 1733, 1417, 1307, 1212, 1175, 1036, 969, 878, 735. HRMS (ESI): [M+Na] ${ }^{+}$calcd for $\mathrm{C}_{17} \mathrm{H}_{22} \mathrm{NaO}_{3}$ 297.1461, found: 297.1460.

(E)-(4-(cyclohex-1-en-1-yl)pent-2-en-2-yl)benzene (3u)

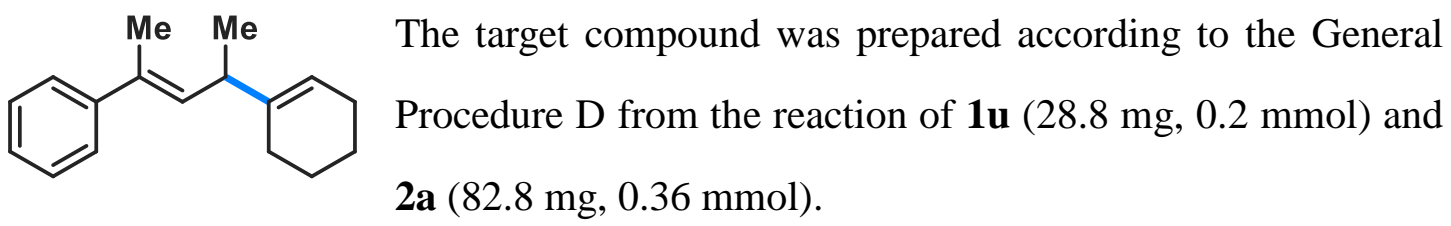

$37.5 \mathrm{mg}, 83 \%$ yield, the regioisomeric ratio of $>20: 1$, colorless oil.

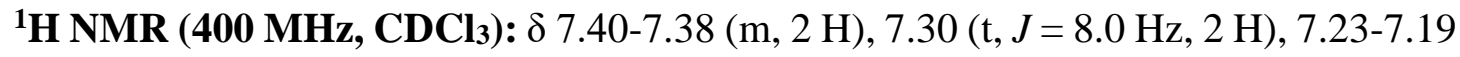


(m, $1 \mathrm{H}), 5.65(\mathrm{dd}, J=8.0,1.2 \mathrm{~Hz}, 1 \mathrm{H}), 5.50(\mathrm{~d}, J=1.2 \mathrm{~Hz}, 1 \mathrm{H}), 3.12-3.04(\mathrm{~m}, 1 \mathrm{H})$, $2.05(\mathrm{~d}, J=1.6 \mathrm{~Hz}, 3 \mathrm{H}), 2.03-1.95(\mathrm{~m}, 4 \mathrm{H}), 1.64-1.52(\mathrm{~m}, 4 \mathrm{H}), 1.14(\mathrm{~d}, J=6.8 \mathrm{~Hz}, 3$ $\mathrm{H})$.

${ }^{13}$ C NMR (100 MHz, CDCl3): $\delta$ 144.0, 141.3, 133.5, 133.3, 128.1, 126.5, 125.7, 119.6, $40.2,26.8,25.3,23.1,22.7,19.3,15.9$.

IR (neat, $\mathbf{c m}^{-1}$ ): 2927, 2835, 1599, 1445, 1378, 1267, 1014, 756, 695.

HRMS (EI): [M] ${ }^{+}$calcd for $\mathrm{C}_{17} \mathrm{H}_{22}$ 226.1722, found: 226.1719 .

(E)-(3-(cyclohex-1-en-1-yl)-2-methylbut-1-en-1-yl)benzene (3v)<smiles>CC(=Cc1ccccc1)C(C)C1=CCCCC1</smiles>
The target compound was prepared according to the General Procedure D from the reaction of $\mathbf{1 v}(28.8 \mathrm{mg}, 0.2 \mathrm{mmol})$ and $2 \mathbf{a}(82.8 \mathrm{mg}, 0.36 \mathrm{mmol})$.

$18.5 \mathrm{mg}, 41 \%$ yield, the regioisomeric ratio of $>20: 1$, colorless oil.

${ }^{1}$ H NMR (400 MHz, CDCl $)$ ): $\delta$ 7.33-7.29 (m, 2 H), 7.26-7.24 (m, 2 H), 7.19-7.15 (m, $1 \mathrm{H}), 6.31(\mathrm{~s}, 1 \mathrm{H}), 5.54-5.52(\mathrm{~m}, 1 \mathrm{H}), 2.78(\mathrm{~d}, J=8.0 \mathrm{~Hz}, 1 \mathrm{H}), 2.07-2.04(\mathrm{~m}, 2 \mathrm{H})$, 2.00-1.91 (m, $1 \mathrm{H}), 1.85-1.76(\mathrm{~m}, 1 \mathrm{H}), 1.73(\mathrm{~d}, J=1.2 \mathrm{~Hz}, 3 \mathrm{H}), 1.62-1.52(\mathrm{~m}, 4 \mathrm{H})$, $1.20(\mathrm{~d}, J=6.8 \mathrm{~Hz}, 3 \mathrm{H})$.

${ }^{13}$ C NMR (100 MHz, CDCl3): $\delta$ 142.0, 139.8, 138.8, 128.9, 128.0, 125.8, 124.7, 121.2, $50.1,27.0,25.4,23.2,22.7,17.1,15.5$.

IR (neat, cm$^{-1}$ ): 2930, 2859, 1643, 1600, 1419, 1210, 1146, 1034, 979, 884, 697.

HRMS (EI): $[\mathrm{M}]{ }^{+}$calcd for $\mathrm{C}_{17} \mathrm{H}_{22} 226.1722$, found: 226.1719 .

\section{(E)-1-(4,8-dimethylnona-3,7-dien-2-yl)cyclopent-1-ene (3w)}

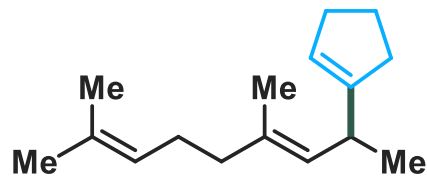

The target compound was prepared according to the General Procedure D from the reaction of 1a $(30.0 \mathrm{mg}, 0.2$ $\mathrm{mmol})$ and $2 \mathbf{d}(77.8 \mathrm{mg}, 0.36 \mathrm{mmol})$.

$36.2 \mathrm{mg}, 83 \%$ yield, the regioisomeric ratio of $>20: 1$, colorless oil.

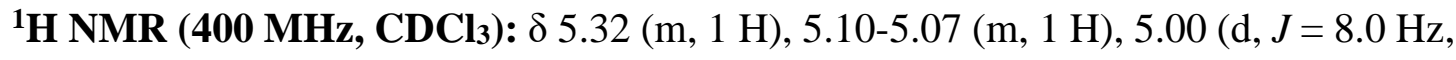
$1 \mathrm{H}), 3.11-3.04(\mathrm{~m}, 1 \mathrm{H}), 2.30-2.22(\mathrm{~m}, 4 \mathrm{H}), 2.08$ (d, $J=8.0 \mathrm{~Hz}, 2 \mathrm{H}), 2.01-1.97$ (m, 2 
H), 1.86-1.79 (m, $2 \mathrm{H}), 1.68(\mathrm{~s}, 3 \mathrm{H}), 1.61(\mathrm{~d}, J=8.0 \mathrm{~Hz}, 6 \mathrm{H}), 1.07$ (d, $J=6.8 \mathrm{~Hz}, 3$ $\mathrm{H})$.

${ }^{13}$ C NMR (100 MHz, CDCl3): $\delta$ 149.1, 133.7, 131.3, 129.2, 124.4, 121.6, 39.7, 34.2, $33.5,32.3,26.6,25.7,23.4,19.8,17.7,16.1$.

IR (neat, $\mathbf{c m}^{-1}$ ): 2963, 2927, 2848, 1451, 1378, 1270, 1091, 1039, 845.

HRMS (EI): [M] ${ }^{+}$calcd for $\mathrm{C}_{16} \mathrm{H}_{26}$ 218.2035, found: 218.2032.

(E)-1-(4,8-dimethylnona-3,7-dien-2-yl)cyclohept-1-ene (3x)

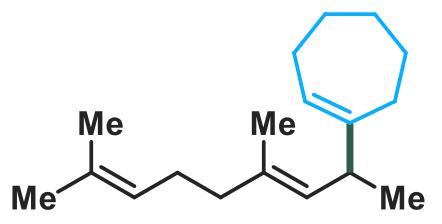

The target compound was prepared according to the General Procedure D from the reaction of $1 \mathbf{a}(30.0 \mathrm{mg}, 0.2$ $30.5 \mathrm{mg}, 62 \%$ yield, the regioisomeric ratio of $>20$ : 1 , colorless oil.

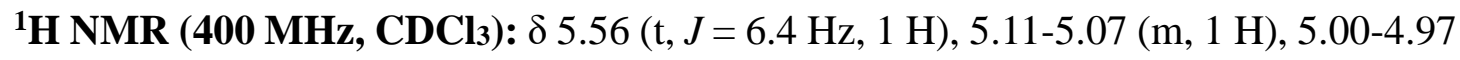
(m, $1 \mathrm{H}), 2.96-2.89$ (m, $1 \mathrm{H}), 2.09-2.04$ (m, $6 \mathrm{H}), 2.00-1.96$ (m, $2 \mathrm{H}), 1.74-1.69$ (m, 2 H), 1.68 (s, $3 \mathrm{H}), 1.61-1.58(\mathrm{~m}, 6 \mathrm{H}), 1.45-1.38(\mathrm{~m}, 4 \mathrm{H}), 1.00(\mathrm{~d}, J=6.8 \mathrm{~Hz}, 3 \mathrm{H})$.

${ }^{13}$ C NMR (100 MHz, CDCl3): $\delta$ 148.8, 134.2, 131.2, 129.0, 124.4, 124.0, 41.1, 39.8, 33.0, 30.9, 28.2, 27.4, 27.3, 26.6, 25.7, 19.8, 17.7, 16.1 .

IR (neat, cm$^{-1}$ ): 2965, 2919, 2849, 1663, 1448, 1349, 1166, 1096, 946, 845, 815, 679. HRMS (EI): [M] ${ }^{+}$calcd for $\mathrm{C}_{18} \mathrm{H}_{30} 246.2348$, found: 246.2345 .

$(E)-1-((S, E)-4,8-d i m e t h y l n o n a-3,7-d i e n-2-y l) c y c l o o c t-1-e n e ~(3 y)$

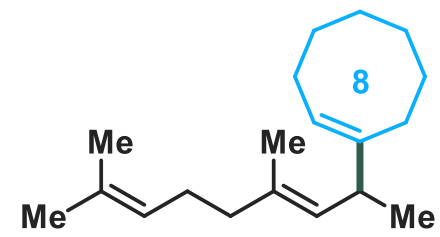

The target compound was prepared according to the General Procedure D from the reaction of $\mathbf{1 a}(30.0 \mathrm{mg}, 0.2$ mmol) and $2 \mathbf{f}(92.9 \mathrm{mg}, 0.36 \mathrm{mmol})$.

$35.9 \mathrm{mg}, 69 \%$ yield, the regioisomeric ratio of $>20: 1$,

colorless oil.

${ }^{1}$ H NMR (400 MHz, CDCl3): $\delta 5.36(\mathrm{td}, J=8.0,0.8 \mathrm{~Hz}, 1 \mathrm{H}), 5.11-5.07(\mathrm{~m}, 1 \mathrm{H})$, 5.00-4.97 (m, $1 \mathrm{H}), 3.02-2.95$ (m, $1 \mathrm{H}), 2.15-2.05(\mathrm{~m}, 6 \mathrm{H}), 2.00-1.96(\mathrm{~m}, 2 \mathrm{H}), 1.67$ (d, $J=1.2 \mathrm{~Hz}, 3 \mathrm{H}), 1.62(\mathrm{~d}, J=1.2 \mathrm{~Hz}, 3 \mathrm{H}), 1.60(\mathrm{~s}, 3 \mathrm{H}), 1.52-1.45(\mathrm{~m}, 8 \mathrm{H}), 1.04(\mathrm{~d}, J$ 
$=6.8 \mathrm{~Hz}, 3 \mathrm{H})$.

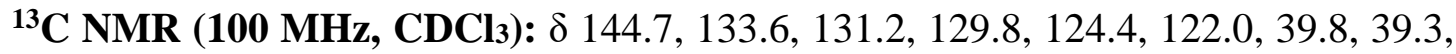
$29.8,29.7,27.8,26.6,26.5,26.4,26.2,25.7,20.1,17.7,16.1$.

IR (neat, $\mathbf{c m}^{-1}$ ): 2924, 2853, 1716, 1449, 1378, 1089, 839, 594.

HRMS (EI): [M] ${ }^{+}$calcd for $\mathrm{C}_{19} \mathrm{H}_{32} 260.2504$, found: 260.2504 .

(E)-1-((S,E)-4,8-dimethylnona-3,7-dien-2-yl)cyclododec-1-ene (3z)

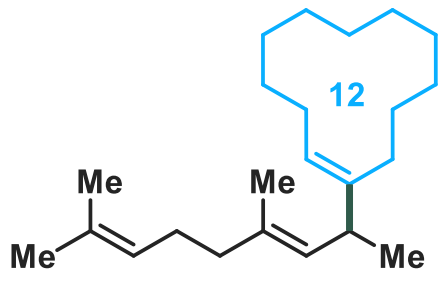

The target compound was prepared according to the General Procedure D from the reaction of $\mathbf{1 a}(30.0 \mathrm{mg}, 0.2$ $\mathrm{mmol}$ ) and $2 \mathrm{~g}$ (113.1 $\mathrm{mg}, 0.36 \mathrm{mmol}$, the stereoisomer ratio of 1:1.32 with $Z / E$ configuration not identified).

$29.7 \mathrm{mg}, 47 \%$ yield, both $Z-\mathbf{2 g}$ and $E-\mathbf{2 g}$ gave the product with branched selectivity (the regioisomeric ratio of $>20: 1)$, colorless oil. The $Z / E$ configuration was not identified. ${ }^{1} \mathbf{H}$ NMR (400 MHz, $\mathbf{C D C l}_{3}$, mixture of $\mathbf{Z} / \mathbf{E}$ isomers): $\delta 5.31-5.14(\mathrm{~m}, 1 \mathrm{H})$, 5.12$5.07(\mathrm{~m}, 1 \mathrm{H}), 4.95(\mathrm{~m}, 1 \mathrm{H}), 3.56-2.96(\mathrm{~m}, 1 \mathrm{H}), 2.21-1.94(\mathrm{~m}, 8 \mathrm{H}), 1.67(\mathrm{~s}, 3 \mathrm{H})$, 1.62-1.60 (m, $6 \mathrm{H}), 1.52-1.39$ (m, $7 \mathrm{H}), 1.35-1.25$ (m, $9 \mathrm{H}), 1.05-1.03$ (m, $3 \mathrm{H})$.

${ }^{13} \mathrm{C}$ NMR (100 MHz, $\mathbf{C D C l}_{3}$, mixture of $\mathbf{Z} / \mathbf{E}$ isomers): $\delta 143.4,141.0,133.3,133.2$, $131.23,131.2,130.9,128.7,126.0,124.5,124.4,123.7,39.9,39.8,36.5,33.5,31.7$, 27.5, 27.3, 26.8, 26.7, 26.66, 26.2, 25.9, 25.8, 25.7, 25.6, 25.4, 25.1, 25.0, 24.9, 24.4, $24.37,24.3,23.6,22.8,22.5,20.7,20.2,17.7,17.65,16.2,16.0$.

IR (neat, $\mathbf{c m}^{-1}$ ): 2924, 2860, 1637, 1467, 1442, 1375, 1108, 983, 726.

HRMS (EI): [M] ${ }^{+}$calcd for $\mathrm{C}_{23} \mathrm{H}_{40} 316.3130$, found: 316.3133 .

Tert-butyl((3-((E)-4,8-dimethylnona-3,7-dien-2-yl)cyclohex-2-en-1-yl)oxy)dimethylsilane (3aa)

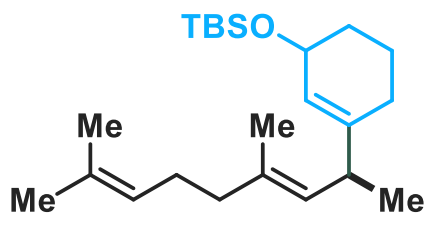

The target compound was prepared according to the General Procedure D from the reaction of 1a $(30.0 \mathrm{mg}, 0.2$ mmol) and $2 \mathbf{h}$ (129.6 mg, $0.36 \mathrm{mmol})$.

$61.6 \mathrm{mg}, 85 \%$ yield, the regioisomeric ratio of $>20: 1$, colorless oil. 
${ }^{1}$ H NMR (400 MHz, CDCl $): \delta 5.37$ (s, $\left.1 \mathrm{H}\right), 5.09-5.06$ (m, $\left.1 \mathrm{H}\right), 5.01$ (d, $J=8.0 \mathrm{~Hz}$, $1 \mathrm{H}), 4.24$ (s, $1 \mathrm{H}), 2.95-2.88$ (m, $1 \mathrm{H}), 2.10-2.04$ (m, $2 \mathrm{H}), 1.99-1.96$ (m, $3 \mathrm{H}), 1.81$ 1.74 (m, $3 \mathrm{H}), 1.67$ (s, $3 \mathrm{H}), 1.61-1.60$ (m, $6 \mathrm{H}), 1.50-1.45$ (m, $2 \mathrm{H}), 1.03$ (d, J = 6.8 Hz, $3 \mathrm{H}), 0.90(\mathrm{~s}, 9 \mathrm{H}), 0.07$ (d, $J=1.6 \mathrm{~Hz}, 6 \mathrm{H})$.

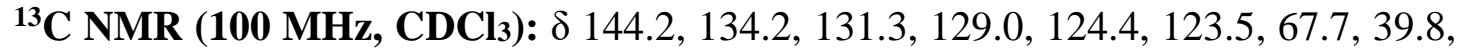
$39.1,32.7,26.6,26.5,26.0,25.7,20.2,19.2,18.4,17.7,16.1,-4.39,-4.44$.

IR (neat, cm$^{-1}$ ): 2957, 2930, 2857, 1422, 1252, 1212, 1144, 1078, 1023, 896, 836, 774. HRMS (ESI): $[\mathrm{M}+\mathrm{Na}]^{+}$calcd for $\mathrm{C}_{23} \mathrm{H}_{42} \mathrm{NaOSi} 385.2897$, found: 385.2905.

\section{3-((E)-4,8-dimethylnona-3,7-dien-2-yl)cyclohex-2-en-1-ol (3ab)}

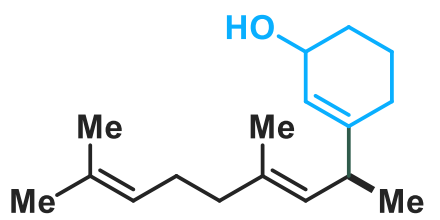

The target compound was prepared according to the General Procedure D from the reaction of $1 \mathbf{a}(30.0 \mathrm{mg}, 0.2$ $\mathrm{mmol})$ and $2 \mathbf{i}(88.6 \mathrm{mg}, 0.36 \mathrm{mmol})$. After $48 \mathrm{~h}$, TBAF (52.3 $\mathrm{mg}, 0.2 \mathrm{mmol}$ ) was added and the reaction was stirred at room temperature for additional $30 \mathrm{~min}$. The solvent was removed, and the residue was purified by flash chromatography on silica gel to afford the desired product.

$29.8 \mathrm{mg}$, $60 \%$ yield, the regioisomeric ratio of $>20: 1$, colorless oil.

${ }^{1}$ H NMR (400 MHz, CDCl3): $\delta 5.38$ (d, $\left.J=1.6 \mathrm{~Hz}, 1 \mathrm{H}\right), 5.09-5.06$ (m, $\left.1 \mathrm{H}\right), 4.99$ 4.96 (m, 1 H), 4.21 (s, 1 H), 2.97-2.89 (m, 1 H), 2.10-2.04 (m, 2 H), 2.01-1.97 (m, 3 H), 1.94-1.72 (m, $3 \mathrm{H}), 1.68$ (s, $3 \mathrm{H}), 1.61-1.52(\mathrm{~m}, 8 \mathrm{H}), 1.40(\mathrm{br}, 1 \mathrm{H}), 1.05(\mathrm{~d}, J=8.0 \mathrm{~Hz}$, $3 \mathrm{H})$.

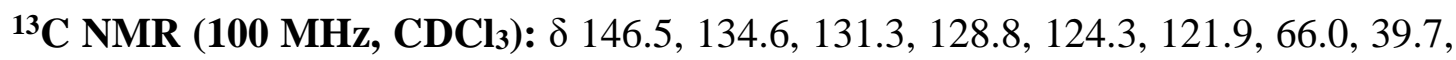
$39.1,32.1,26.8,26.5,25.7,19.3,19.2,17.7,16.1$.

IR (neat, $\mathbf{c m}^{-1}$ ): 3418, 2929, 2855, 1638, 1453, 1127, 1053, 968, 779.

HRMS (ESI): [M+Na] ${ }^{+}$calcd for $\mathrm{C}_{17} \mathrm{H}_{28} \mathrm{NaO}$ 271.2032, found: 271.2030. 
(c)

colorless oil.
The target compound was prepared according to the General Procedure D from the reaction of $1 \mathbf{a}(30.0 \mathrm{mg}, 0.2$ $\mathrm{mmol})$ and $\mathbf{2 j}$ (103.7 mg, $0.36 \mathrm{mmol})$.

$51.1 \mathrm{mg}, 88 \%$ yield, the regioisomeric ratio of $>20: 1$,

${ }^{1}$ H NMR (400 MHz, CDCl $)$ : $\delta 5.33(\mathrm{~d}, J=1.2 \mathrm{~Hz}, 1 \mathrm{H}), 5.09-5.06(\mathrm{~m}, 1 \mathrm{H}), 4.97(\mathrm{dd}$, $J=8.0,0.8 \mathrm{~Hz}, 1 \mathrm{H}), 3.97(\mathrm{~s}, 4 \mathrm{H}), 2.98-2.90(\mathrm{~m}, 1 \mathrm{H}), 2.27(\mathrm{~s}, 2 \mathrm{H}), 2.23-2.13(\mathrm{~m}, 2$ H), 2.10-2.05 (m, 2 H), 2.01-1.97 (m, 2 H), 1.75-1.72 (m, 2 H), 1.67 (s, 3 H), 1.61-1.60 (m, $6 \mathrm{H}), 1.05(\mathrm{~d}, J=6.8 \mathrm{~Hz}, 3 \mathrm{H})$.

${ }^{13}$ C NMR (100 MHz, CDCl3): $\delta$ 141.4, 134.2, 131.2, 129.3, 124.3, 116.0, 108.3, 64.3, $39.7,38.5,35.8,31.4,26.5,26.1,25.7,19.3,17.7,16.1$.

IR (neat, cm-1): 2963, 2926, 1725, 1670, 1449, 1378, 1259, 1211, 1117, 1061, 864.

HRMS (ESI): $[\mathrm{M}+\mathrm{H}]^{+}$calcd for $\mathrm{C}_{19} \mathrm{H}_{31} \mathrm{O}_{2}$ 291.2319, found: 291.2317.

Ethyl 4-((E)-4,8-dimethylnona-3,7-dien-2-yl)cyclohex-3-ene-1-carboxylate (3ad)

General Procedure $\mathrm{D}$ from the reaction of $1 \mathrm{a}(30.0 \mathrm{mg}, 0.2$
$\mathrm{mmol})$ and $\mathbf{2 c}(108.7 \mathrm{mg}, 0.36 \mathrm{mmol})$. 1.1:1, colorless oil.

${ }^{1}$ H NMR (400 MHz, CDCl3): $\delta 5.41(\mathrm{~s}, 1 \mathrm{H}), 5.09-5.06(\mathrm{~m}, 1 \mathrm{H}), 4.95(\mathrm{~d}, J=12.0 \mathrm{~Hz}$, $1 \mathrm{H}), 4.13(\mathrm{q}, J=8.0 \mathrm{~Hz}, 2 \mathrm{H}), 2.94-2.85(\mathrm{~m}, 1 \mathrm{H}), 2.50-2.42(\mathrm{~m}, 1 \mathrm{H}), 2.25-2.23(\mathrm{~m}, 2$ H), 2.08-1.96 (m, 7 H), 1.69-1.63 (m, 4 H), 1.62-1.57 (m, $6 \mathrm{H}), 1.25$ (t, $J=8.0 \mathrm{~Hz}, 3$ H), $1.02(\mathrm{~d}, J=6.8 \mathrm{~Hz}, 3 \mathrm{H})$.

${ }^{13}$ C NMR (100 MHz, CDCl3): $\delta$ 176.1, [141.7, 141.5], [134.3, 134.2], 131.3, [129.3, 129.2], 124.3, [117.1, 117.0], 60.1, 39.7, 39.1, 38.8, 27.8, 26.6, 26.1, 26.0, 25.7, 19.3, $17.7,16.1,14.2$.

IR (neat, cm-1): 2966, 2928, 1735, 1451, 1376, 1308, 1225, 1170, 1097, 1033, 811. 
HRMS (ESI): $[\mathrm{M}+\mathrm{H}]^{+}$calcd for $\mathrm{C}_{20} \mathrm{H}_{33} \mathrm{O}_{2}$ 305.2475, found: 305.2477 .

\section{4-(tert-butyl)-1-((E)-4,8-dimethylnona-3,7-dien-2-yl)cyclohex-1-ene (3ae)}<smiles>CC(C)=CCCC(C)=CC(C)C1=CCC(C(=O)c2ccccc2)CC1</smiles>

The target compound was prepared according to the General Procedure D from the reaction of 1a $(30.0 \mathrm{mg}, 0.2$ mmol) and 2k (103.0 mg, $0.36 \mathrm{mmol})$.

$45.5 \mathrm{mg}, 79 \%$ yield, the regioisomeric ratio of $>20: 1, \mathrm{dr}=$

2:1, colorless oil.

${ }^{1}$ H NMR (400 MHz, CDCl3): $\delta 5.42(\mathrm{~s}, 1 \mathrm{H}$ ), 5.10-5.06 (m, $1 \mathrm{H}), 4.97(\mathrm{~d}, J=8.0 \mathrm{~Hz}$, $1 \mathrm{H}), 2.94-2.86$ (m, $1 \mathrm{H}), 2.10-1.92$ (m, $7 \mathrm{H}), 1.82-1.74$ (m, $2 \mathrm{H}), 1.67$ (s, $3 \mathrm{H}), 1.62-$ $1.60(\mathrm{~m}, 6 \mathrm{H}), 1.26-1.05(\mathrm{~m}, 2 \mathrm{H}), 1.02(\mathrm{~d}, J=6.8 \mathrm{~Hz}, 3 \mathrm{H}), 0.85(\mathrm{~s}, 9 \mathrm{H})$.

${ }^{13}$ C NMR (100 MHz, CDCl3): $\delta$ [141.9, 141.7], [133.8, 133.6], 131.2, [129.8 129.6], 124.4, [119.1, 119.0], 44.4, 39.8, [39.1, 38.8], 32.2, [28.3, 28.1], 27.2, 26.8, 26.6, 25.7, $[24.5,24.4],[19.7,19.4], 17.7,[16.10,16.06]$.

IR (neat, $\mathbf{c m}^{-1}$ ): 2962, 2918, 1657, 1452, 1364, 1090, 1022, 810.

HRMS (EI): [M] ${ }^{+}$calcd for $\mathrm{C}_{21} \mathrm{H}_{36} 288.2817$, found: 288.2812 .

\section{(E)-4-(4,8-dimethylnona-3,7-dien-2-yl)-1-tosyl-1,2,3,6-tetrahydropyridine (3af)}

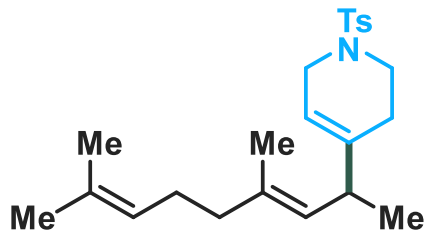

The target compound was prepared according to the General Procedure D from the reaction of 1a $(30.0 \mathrm{mg}, 0.2$ mmol) and $2 \mathbf{b}$ (138.6 mg, $0.36 \mathrm{mmol}) ; 65.8 \mathrm{mg}, 85 \%$ yield, the regioisomeric ratio of $>20: 1$, colorless oil.

Gram-scale reaction: The target compound $(1.3 \mathrm{~g}, 84 \%$ yield, the regioisomeric ratio of >20:1) was prepared according to the General Procedure D. Ni(COD) 2 (55.0 mg, 0.2 mmol), L9 (122.6 mg, 0.24 mmol), CsF (1.2 g, 8.0 mmol), PMHS (2.2 g, 10.0 mmol), dienes $1 \mathbf{a}(0.6 \mathrm{~g}, 4.0 \mathrm{mmol})$, vinyl triflates $\mathbf{2 b}(2.77 \mathrm{~g}, 7.2 \mathrm{mmol})$, and THF/DIPE (3:1, $20 \mathrm{~mL}, 0.2 \mathrm{M}$ ) were used.

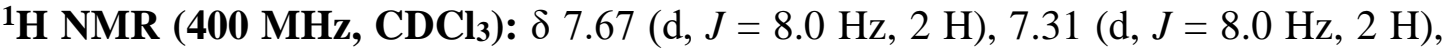
$5.32(\mathrm{~s}, 1 \mathrm{H}), 5.05-5.02(\mathrm{~m}, 1 \mathrm{H}), 4.85(\mathrm{~d}, J=8.0 \mathrm{~Hz}, 1 \mathrm{H}), 3.62-3.50(\mathrm{q}, J=16.0 \mathrm{~Hz}$, 
$2 \mathrm{H}), 3.21-3.05$ (m, 2 H), 2.92-2.84 (m, 1 H), 2.42 (s, 3 H), 2.12-2.02 (m, 4 H), 1.97$1.93(\mathrm{~m}, 2 \mathrm{H}), 1.66(\mathrm{~s}, 3 \mathrm{H}), 1.56(\mathrm{~d}, J=8.0 \mathrm{~Hz}, 6 \mathrm{H}), 0.99$ (d, $J=6.8 \mathrm{~Hz}, 3 \mathrm{H})$.

${ }^{13}$ C NMR (100 MHz, CDCl3): $\delta$ 143.4, 140.6, 135.2, 133.3, 131.4, 129.5, 128.1, 127.7, $124.1,114.4,44.9,43.1,39.6,38.4,26.8,26.5,25.7,21.5,19.0,17.7,16.1$.

IR (neat, $\mathbf{c m}^{-1}$ ): 2963, 2923, 1671, 1453, 1347, 1165, 1096, 944, 817, 679, 551.

HRMS (ESI): $[\mathrm{M}+\mathrm{H}]^{+}$calcd for $\mathrm{C}_{23} \mathrm{H}_{34} \mathrm{NO}_{2} \mathrm{~S} 388.2305$, found: 388.2310 .

\section{(E)-4-(4,8-dimethylnona-3,7-dien-2-yl)-3,6-dihydro-2H-pyran (3ag)}

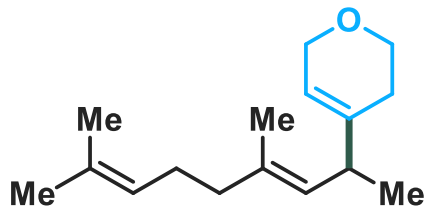

The target compound was prepared according to the General Procedure D from the reaction of 1a $(30.0 \mathrm{mg}, 0.2$ $37.9 \mathrm{mg}, 81 \%$ yield, the regioisomeric ratio of $>20: 1$, colorless oil.

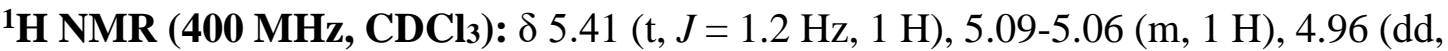
$J=8.0,1.2 \mathrm{~Hz}, 1 \mathrm{H}), 4.14-4.13$ (m, $2 \mathrm{H}), 3.81-3.70$ (m, $2 \mathrm{H}), 2.96-2.89$ (m, $1 \mathrm{H}), 2.11-$ $1.98(\mathrm{~m}, 6 \mathrm{H}), 1.67(\mathrm{~s}, 3 \mathrm{H}), 1.62-1.56(\mathrm{~m}, 6 \mathrm{H}), 1.06(\mathrm{~d}, J=6.8 \mathrm{~Hz}, 3 \mathrm{H})$.

${ }^{13}$ C NMR (100 MHz, CDCl3): $\delta$ 139.6, 134.8, 131.3, 128.5, 124.2, 117.8, 65.7, 64.5, $39.7,38.5,27.0,26.5,25.7,18.9,17.7,16.1$

IR (neat, cm$^{-1}$ ): 2963, 2925, 2851, 1642, 1452, 1382, 1133, 1041, 850, 813, 750.

HRMS (EI): $[\mathrm{M}]^{+}$calcd for $\mathrm{C}_{16} \mathrm{H}_{26} \mathrm{O} 234.1984$, found: 234.1982 .

\section{(E)-4-(4,8-dimethylnona-3,7-dien-2-yl)-3,6-dihydro-2H-thiopyran (3ah)}

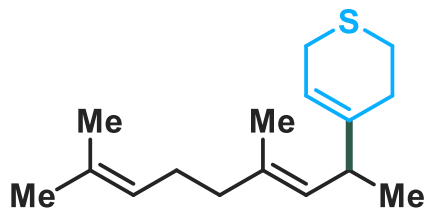

The target compound was prepared according to the General Procedure D from the reaction of 1a $(30.0 \mathrm{mg}, 0.2$ $36.0 \mathrm{mg}, 72 \%$ yield, the regioisomeric ratio of $>20: 1$, colorless oil

${ }^{1}$ H NMR (400 MHz, CDCl $): \delta 5.62-5.60(\mathrm{~m}, 1 \mathrm{H}), 5.09-5.05(\mathrm{~m}, 1 \mathrm{H}), 4.96(\mathrm{dd}, J=$ 8.0, $1.2 \mathrm{~Hz}, 1 \mathrm{H}), 3.15(\mathrm{~d}, J=2.0 \mathrm{~Hz}, 2 \mathrm{H}), 2.94-2.87(\mathrm{~m}, 1 \mathrm{H}), 2.70(\mathrm{t}, J=4.0 \mathrm{~Hz}, 2$ H), 2.30-2.15 (m, 2 H), 2.10-2.05 (m, 2 H), 2.01-1.97 (m, 2 H), 1.68 (s, 3 H), 1.61-1.60 $(\mathrm{m}, 6 \mathrm{H}), 1.05(\mathrm{~d}, J=6.8 \mathrm{~Hz}, 3 \mathrm{H})$ 
${ }^{13}$ C NMR (100 MHz, CDCl $): \delta$ 142.9, 134.8, 131.3, 128.9, 124.2, 116.0, 40.1, 39.7, 27.5, 26.5, 25.7 (2 C), 25.4, 19.3, 17.7, 16.2.

IR (neat, $\mathbf{c m}^{-1}$ ): 2963, 2918, 1650, 1421, 1379, 1211, 1142, 1029, 855, 751.

HRMS (EI): [M] ${ }^{+}$calcd for $\mathrm{C}_{16} \mathrm{H}_{26} \mathrm{~S} 250.1755$, found: 250.1752 .

\section{4-((E)-4,8-dimethylnona-3,7-dien-2-yl)-1,2,3,8a-tetrahydronaphthalene (3ai)}

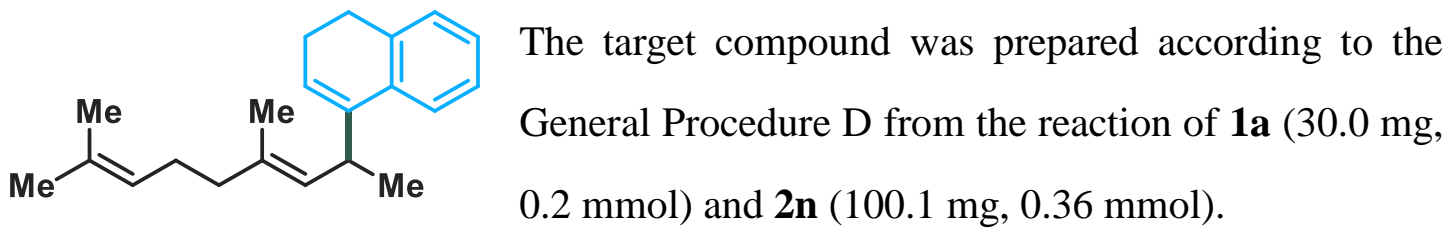

$39.8 \mathrm{mg}, 71 \%$ yield, the regioisomeric ratio of $>20: 1$, colorless oil.

${ }^{1} \mathbf{H}$ NMR (600 MHz, CDCl3): $\delta 7.28$ (d, $J=7.8$ Hz, $1 \mathrm{H}$ ), 7.19-7.16 (m, $\left.1 \mathrm{H}\right)$, 7.137.09 (m, 2 H), 5.91 (t, $J=4.2 \mathrm{~Hz}, 1 \mathrm{H}), 5.14-5.12(\mathrm{~m}, 1 \mathrm{H}), 5.07-5.05(\mathrm{~m}, 1 \mathrm{H}), 3.62-$ 3.57 (m, $1 \mathrm{H}), 2.70$ (t, $J=7.8 \mathrm{~Hz}, 2 \mathrm{H}), 2.25-2.22$ (m, $2 \mathrm{H}), 2.10-2.06$ (m, $2 \mathrm{H}), 2.02-$ 1.99 (m, 2 H), 1.68-1.65 (m, $6 \mathrm{H}), 1.58$ (s, $3 \mathrm{H}), 1.18$ (d, J = 6.6 Hz, $3 \mathrm{H})$.

${ }^{13}$ C NMR (100 MHz, CDCl3): $\delta$ 141.1, 137.0, 135.2, 134.3, 131.3, 130.2, 127.5, 126.2, $126.1,124.30,124.26,122.9,39.6,33.4,28.5,26.5,25.7,23.2,20.7,17.7,16.1$.

IR (neat, $\mathbf{c m}^{-1}$ ): 2964, 2929, 1633, 1486, 1450, 1378, 1275, 1106, 1022, 823, 782, 765. GC-MS (EI): $[\mathrm{M}]^{+}$calcd for $\mathrm{C}_{21} \mathrm{H}_{28}$ 280.22, found: 280.16 .

\section{(E)-2,6,8-trimethyl-9-methylenetetradeca-2,6-diene (3aj)}

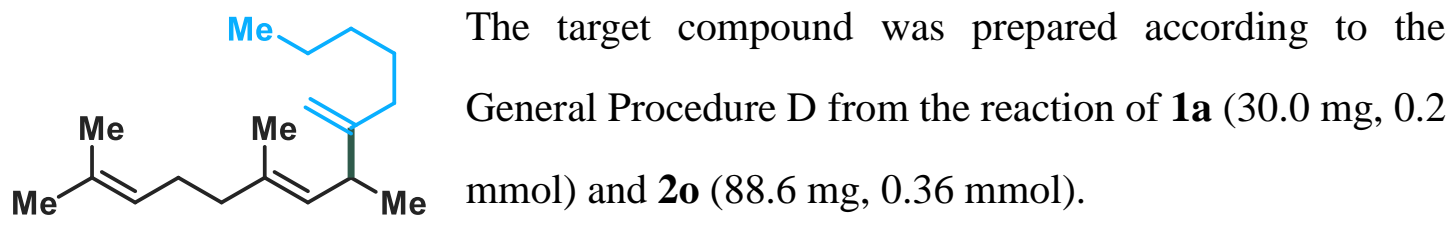

$31.3 \mathrm{mg}, 63 \%$ yield, the regioisomeric ratio of $>20: 1$, colorless oil.

${ }^{1}$ H NMR (400 MHz, CDCl3): $\delta$ 5.10-5.06 (m, $\left.1 \mathrm{H}\right), 4.98-4.95(\mathrm{~m}, 1 \mathrm{H}), 4.76(\mathrm{~s}, 1 \mathrm{H})$, 4.69 (s, $1 \mathrm{H}), 3.03-2.95(\mathrm{~m}, 1 \mathrm{H}), 2.10-2.05(\mathrm{~m}, 2 \mathrm{H}), 2.00-1.96(\mathrm{~m}, 4 \mathrm{H}), 1.67(\mathrm{~d}, J=$ $0.8 \mathrm{~Hz}, 3 \mathrm{H}), 1.62-1.60$ (m, $6 \mathrm{H}), 1.47-1.38(\mathrm{~m}, 2 \mathrm{H}), 1.36-1.23(\mathrm{~m}, 4 \mathrm{H}), 1.06$ (d, $J=$ $6.8 \mathrm{~Hz}, 3 \mathrm{H}), 0.89$ (t, $J=8.0 \mathrm{~Hz}, 3 \mathrm{H})$.

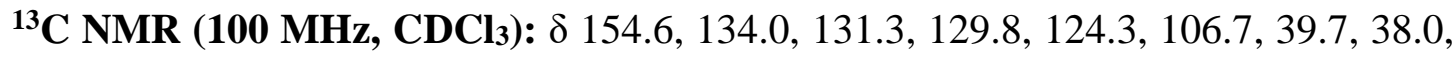


$34.7,31.8,27.8,26.6,25.7,22.6,19.9,17.7,16.1,14.1$.

IR (neat, $\mathbf{c m}^{-1}$ ): 2961, 2928, 2858, 1642, 1453, 1379, 1106, 1054, 889.

HRMS (EI): [M] ${ }^{+}$calcd for $\mathrm{C}_{18} \mathrm{H}_{32} 248.2504$, found: 248.2501 .

(E)-(4,6,10-trimethyl-3-methyleneundeca-5,9-dien-1-yl)benzene (3ak)

The target compound was prepared according to the
General Procedure $\mathrm{D}$ from the reaction of $1 \mathbf{a}(30.0 \mathrm{mg}, 0.2$ $44.6 \mathrm{mg}, 79 \%$ yield, the regioisomeric ratio of $>20: 1$, colorless oil.

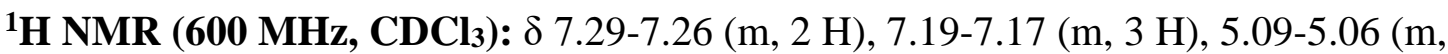
$1 \mathrm{H}), 4.99-4.98(\mathrm{~m}, 1 \mathrm{H}), 4.83(\mathrm{~s}, 1 \mathrm{H}), 4.77(\mathrm{~s}, 1 \mathrm{H}), 3.07-3.02(\mathrm{~m}, 1 \mathrm{H}), 2.79-2.70(\mathrm{~m}$, $2 \mathrm{H}), 2.35-2.26$ (m, $2 \mathrm{H}), 2.10-2.05$ (m, $2 \mathrm{H}), 2.01-1.98$ (m, $2 \mathrm{H}), 1.65-1.63$ (m, $6 \mathrm{H})$, $1.59(\mathrm{~s}, 3 \mathrm{H}), 1.08(\mathrm{~d}, J=6.6 \mathrm{~Hz}, 3 \mathrm{H})$.

${ }^{13}$ C NMR (150 MHz, CDCl $): \delta 153.7,142.5,134.4,131.4,129.5,128.33,128.26$, $125.7,124.3,107.4,39.7,38.3,36.5,34.8,26.6,25.7,19.8,17.7,16.2$.

IR (neat, cm-1): 2932, 2859, 1471, 1362, 1253, 1093, 1008, 836, 773.

GC-MS (EI): $[\mathrm{M}]^{+}$calcd for $\mathrm{C}_{21} \mathrm{H}_{30}$ 282.23, found: 282.16 .

Methyl (E)-7,9,13-trimethyl-6-methylenetetradeca-8,12-dienoate (3al)

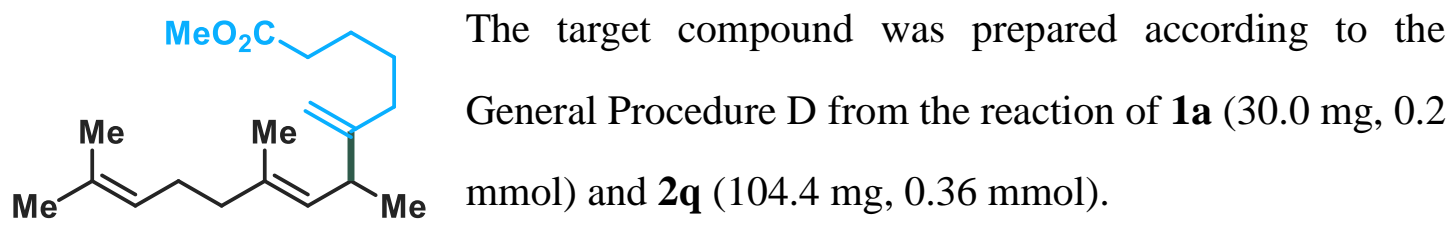

$46.2 \mathrm{mg}, 79 \%$ yield, the regioisomeric ratio of >20:1, colorless oil.

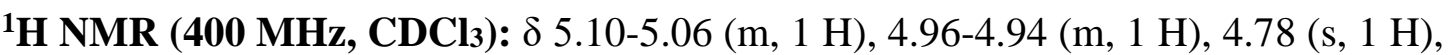
$4.69(\mathrm{~d}, J=0.8 \mathrm{~Hz}, 1 \mathrm{H}), 3.66(\mathrm{~s}, 3 \mathrm{H}), 3.01-2.94(\mathrm{~m}, 1 \mathrm{H}), 2.32(\mathrm{t}, J=8.0 \mathrm{~Hz}, 2 \mathrm{H})$, 2.10-1.97 (m, $6 \mathrm{H}), 1.69(\mathrm{~d}, J=0.8 \mathrm{~Hz}, 3 \mathrm{H}), 1.67-1.60(\mathrm{~m}, 8 \mathrm{H}), 1.50-1.41(\mathrm{~m}, 2 \mathrm{H})$, $1.06(\mathrm{~d}, J=7.2 \mathrm{~Hz}, 3 \mathrm{H})$.

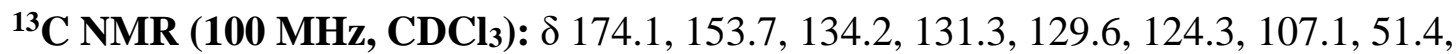
$39.7,37.9,34.3,34.0,27.5,26.6,25.7,24.8,19.8,17.7,16.1$. 
IR (neat, cm$^{-1}$ ): 2929, 2861, 1741, 1643, 1437, 1375, 1238, 1196, 1172, 1055, 889.

HRMS (ESI): [M+Na] ${ }^{+}$calcd for $\mathrm{C}_{19} \mathrm{H}_{32} \mathrm{NaO}_{2}$ 315.2295, found: 315.2292.

\section{(E)-7,9,13-trimethyl-6-methylene- $N$-(p-tolyl)tetradeca-8,12-dienamide (3am)}<smiles>C=C(C)C(C)/C=C(\C)CCCCC(=O)Nc1ccc(C)cc1</smiles>
The target compound was prepared according to the General Procedure D from the reaction of $\mathbf{1 a}(30.0 \mathrm{mg}, 0.2$ mmol) and $2 \mathbf{r}(131.4 \mathrm{mg}, 0.36 \mathrm{mmol})$.

$58.8 \mathrm{mg}, 80 \%$ yield, the regioisomeric ratio of $>20: 1$, colorless oil.

${ }^{1}$ H NMR (400 MHz, CDCl 3$): \delta 7.38(\mathrm{~d}, J=8.0 \mathrm{~Hz}, 2 \mathrm{H}), 7.28(\mathrm{br}, 1 \mathrm{H}), 7.10(\mathrm{~d}, J=$ $8.0 \mathrm{~Hz}, 2 \mathrm{H}), 5.07$ (t, $J=6.8 \mathrm{~Hz}, 1 \mathrm{H}), 4.95(\mathrm{~d}, J=8.0 \mathrm{~Hz}, 1 \mathrm{H}), 4.78(\mathrm{~s}, 1 \mathrm{H}), 4.69$ (s, $1 \mathrm{H}), 3.01-2.94$ (m, $1 \mathrm{H}), 2.35-2.30$ (m, $5 \mathrm{H}), 2.09-1.96$ (m, $6 \mathrm{H}), 1.75-1.70$ (m, $2 \mathrm{H})$, $1.67(\mathrm{~s}, 3 \mathrm{H}), 1.60(\mathrm{~d}, J=8.0 \mathrm{~Hz}, 6 \mathrm{H}), 1.54-1.49(\mathrm{~m}, 2 \mathrm{H}), 1.06(\mathrm{~d}, J=6.8 \mathrm{~Hz}, 3 \mathrm{H})$.

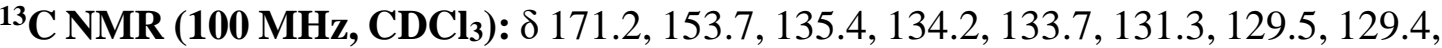
$124.2,119.9,107.2,39.7,38.0,37.6,34.3,27.6,26.6,25.7,25.5,20.8,19.8,17.7,16.1$. IR (neat, $\mathbf{c m}^{-1}$ ): 2963, 2927, 1658, 1604, 1539, 1405, 1377, 1250, 889, 817

HRMS (ESI): $[\mathrm{M}+\mathrm{H}]^{+}$calcd for $\mathrm{C}_{25} \mathrm{H}_{38} \mathrm{NO} 368.2948$, found: 368.2942 .

(E)-7,9,13-trimethyl-6-methylenetetradeca-8,12-dien-1-yl

2-(2-fluoro-[1,1'biphenyl]-4-yl)propanoate (3an)

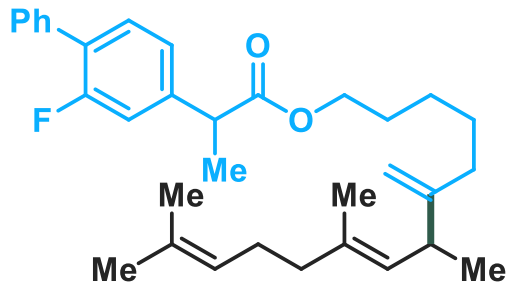

The target compound was prepared according to the General Procedure D from the reaction of $\mathbf{1 a}(30.0 \mathrm{mg}$, $0.2 \mathrm{mmol})$ and $2 \mathrm{~s}(175.7 \mathrm{mg}, 0.36 \mathrm{mmol})$.

colorless oil.

${ }^{1}$ H NMR (400 MHz, CDCl3): $\delta 7.53(\mathrm{~d}, J=8.0 \mathrm{~Hz}, 2 \mathrm{H}), 7.43(\mathrm{t}, J=8.0 \mathrm{~Hz}, 2 \mathrm{H})$, 7.39-7.34 (m, 2 H), 7.16-7.11 (m, 2 H), 5.07 (t, $J=6.8 \mathrm{~Hz}, 1 \mathrm{H}), 4.94(\mathrm{~d}, J=12.0 \mathrm{~Hz}$ $1 \mathrm{H}), 4.76(\mathrm{~s}, 1 \mathrm{H}), 4.66(\mathrm{~s}, 1 \mathrm{H}), 4.11-4.08(\mathrm{~m}, 2 \mathrm{H}), 3.77-3.72(\mathrm{~m}, 1 \mathrm{H}), 2.99-2.92(\mathrm{~m}$, $1 \mathrm{H}), 2.09-2.04$ (m, $2 \mathrm{H}), 1.99-1.95$ (m, $4 \mathrm{H}), 1.67$ (s, $3 \mathrm{H}), 1.64-1.59$ (m, $8 \mathrm{H}), 1.53$ (d, 
$J=8.0 \mathrm{~Hz}, 3 \mathrm{H}), 1.44-1.39(\mathrm{~m}, 2 \mathrm{H}), 1.36-1.26(\mathrm{~m}, 2 \mathrm{H}), 1.04(\mathrm{~d}, J=6.8 \mathrm{~Hz}, 3 \mathrm{H})$.

${ }^{13}$ C NMR (100 MHz, CDCl $): \delta 174.0,159.6(\mathrm{~d}, J=246 \mathrm{~Hz}), 153,9,141.9$ (d, $J=8$ Hz), 135.5, 134.1, 131.3, 130.7 (d, $J=4$ Hz), 129.6, 128.93, 128.90, 128.4 (2 C), 127.6, $124.3,123.5(\mathrm{~d}, J=3 \mathrm{~Hz}), 115.2(\mathrm{~d}, J=23 \mathrm{~Hz}), 107.0,65.1,45.1,39.7,37.9,34.5,28.5$, $27.6,26.6,25.69,25.66,19.8,18.3,17.7,16.1$.

${ }^{19}$ F NMR (376 MHz, CDCl3): $\delta-117.7$.

IR (neat, cm$^{-1}$ ): 2961, 2933, 1736, 1641, 1438, 1379, 1198, 1172, 884.

HRMS (ESI): $[\mathrm{M}+\mathrm{Na}]^{+}$calcd for $\mathrm{C}_{33} \mathrm{H}_{43} \mathrm{FNaO}_{2}$ 513.3139, found: 513.3141 .

\section{(E)-6,8,12-trimethyl-5-methylenetrideca-7,11-dien-1-yl acetate (3ao)}

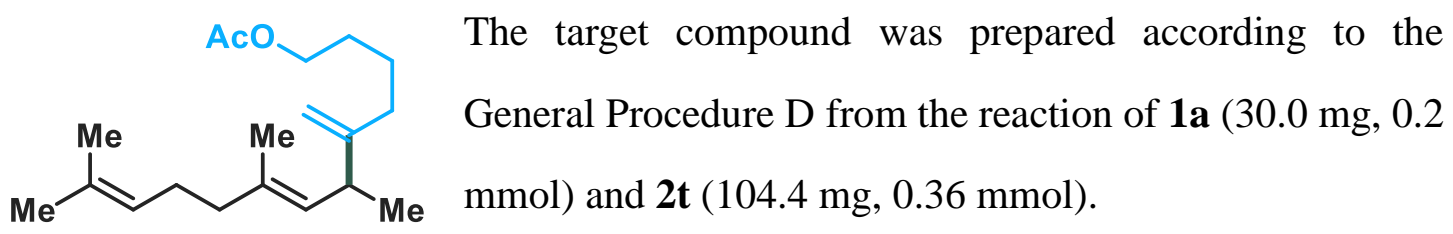

$49.7 \mathrm{mg}, 85 \%$ yield, the regioisomeric ratio of $>20: 1$, colorless oil.

${ }^{1}$ H NMR (400 MHz, CDCl3): $\delta 5.08(\mathrm{tt}, J=8.0,1.6 \mathrm{~Hz}, 1 \mathrm{H}), 4.96(\mathrm{dd}, J=8.0,0.8$ $\mathrm{Hz}, 1 \mathrm{H}), 4.79(\mathrm{~s}, 1 \mathrm{H}), 4.70(\mathrm{~d}, J=1.2 \mathrm{~Hz}, 1 \mathrm{H}), 4.06(\mathrm{t}, J=8.0 \mathrm{~Hz}, 2 \mathrm{H}), 3.02-2.95$ (m, $1 \mathrm{H}), 2.10-1.97$ (m, 9 H), 1.69 (s, $3 \mathrm{H}), 1.67-1.60$ (m, 8 H), 1.54-1.47 (m, $2 \mathrm{H}), 1.06$ $(\mathrm{d}, J=6.8 \mathrm{~Hz}, 3 \mathrm{H})$.

${ }^{13}$ C NMR (100 MHz, CDCl3): $\delta$ 171.2, 153.6, 134.2, 131.3, 129.5, 124.3, 107.3, 64.5, $39.7,37.9,34.2,28.4,26.6,25.7,24.3,21.0,19.8,17.7,16.1$.

IR (neat, $\left.\mathbf{c m}^{-1}\right)$ : 2965, 2929, 2856, 1742, 1646, 1455, 1369, 1240, 1045, 888.

HRMS (ESI): [M+Na ${ }^{+}$calcd for : $\mathrm{C}_{19} \mathrm{H}_{32} \mathrm{NaO}_{2}$ 315.2295, found: 315.2288.

\section{(E)-6,8,12-trimethyl-5-methylenetrideca-7,11-dien-1-ol (3ap)}

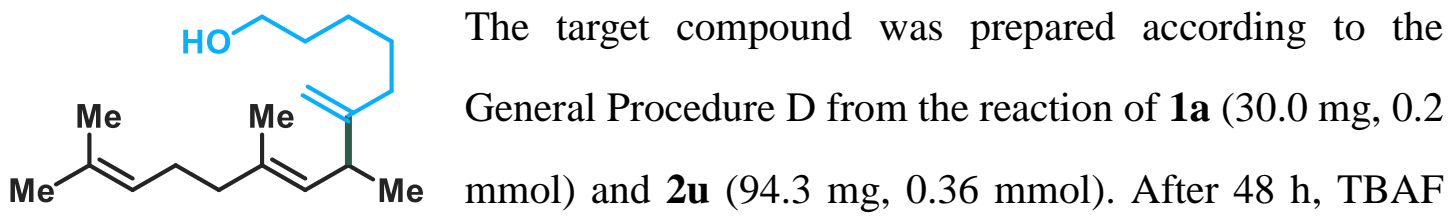

(52.3 $\mathrm{mg}, 0.2 \mathrm{mmol}$ ) was added and the reaction mixture was stirred at room temperature for additional $30 \mathrm{~min}$. The solvent was removed, and the residue was 
purified by flash chromatography on silica gel to afford the desired product.

$32.8 \mathrm{mg}, 62 \%$ yield, the regioisomeric ratio of $>20: 1$, colorless oil.

${ }^{1}$ H NMR (400 MHz, CDCl3): $\delta 5.08(\mathrm{t}, J=8.0 \mathrm{~Hz}, 1 \mathrm{H}), 4.96(\mathrm{~d}, J=8.0 \mathrm{~Hz}, 1 \mathrm{H})$, $4.77(\mathrm{~s}, 1 \mathrm{H}), 4.70(\mathrm{~s}, 1 \mathrm{H}), 3.65(\mathrm{t}, J=6.8 \mathrm{~Hz}, 2 \mathrm{H}), 3.02-2.95(\mathrm{~m}, 1 \mathrm{H}), 2.09-1.97(\mathrm{~m}$, $6 \mathrm{H}), 1.68$ (s, $3 \mathrm{H}), 1.62-1.55$ (m, 7 H), 1.51-1.42 (m, 2 H), 1.40-1.32 (m, 2 H), 1.07 (d, $J=6.8 \mathrm{~Hz}, 3 \mathrm{H}), 0.88-0.83(\mathrm{~m}, 2 \mathrm{H})$.

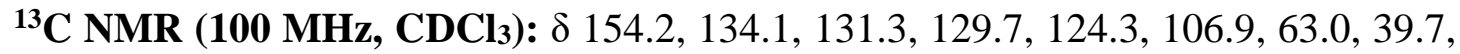
$38.0,34.6,32.7,29.7,27.8,26.6,25.7,19.9,17.7,16.1$.

IR (neat, cm$^{-1}$ ): 3382, 2932, 2858, 2169, 1640, 1451, 1376, 1272, 1053, 891.

HRMS (ESI): $[\mathrm{M}+\mathrm{H}]{ }^{+}$calcd for $\mathrm{C}_{18} \mathrm{H}_{33} \mathrm{O}$ 265.2526, found: 265.2518 .

(2R,8R,8aS)-6-((E)-4,8-dimethylnona-3,7-dien-2-yl)-8,8a-dimethyl-2-(prop-1-en2-yl)-1,2,3,7,8,8a-hexahydronaphthalene (6)

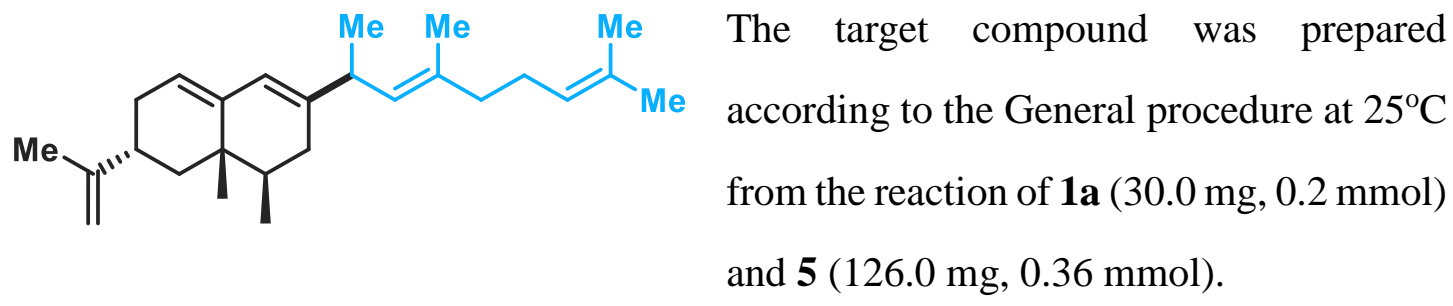

$37.3 \mathrm{mg}, 53 \%$ yield, the regioisomeric ratio of $>20: 1, \mathrm{dr}=1.9: 1$, colorless oil.

${ }^{1}$ H NMR (400 MHz, CDCl3): $\delta 5.77(\mathrm{~s}, 1 \mathrm{H}), 5.38(\mathrm{t}, J=2.4 \mathrm{~Hz}, 1 \mathrm{H}), 5.08(\mathrm{tt}, J=6.8$, $1.6 \mathrm{~Hz}, 1 \mathrm{H})$, 5.03-4.98 (m, $1 \mathrm{H})$, 4.74-4.73 (m, $2 \mathrm{H}), 3.01-2.92$ (m, $1 \mathrm{H})$, 2.45-2.39 (m, $1 \mathrm{H}), 2.26-2.19$ (m, $1 \mathrm{H}), 2.07$ (q, $J=7.2 \mathrm{~Hz}, 2 \mathrm{H}), 2.02-1.94(\mathrm{~m}, 3 \mathrm{H}), 1.91-1.84(\mathrm{~m}, 2$ H), $1.75(\mathrm{~s}, 3 \mathrm{H}), 1.73-1.68(\mathrm{~m}, 5 \mathrm{H}), 1.63(\mathrm{~d}, J=1.2 \mathrm{~Hz}, 2 \mathrm{H}), 1.61-1.58(\mathrm{~m}, 4 \mathrm{H})$, 1.54-1.45 (m, $1 \mathrm{H}), 1.07$ (d, $J=6.8 \mathrm{~Hz}, 3 \mathrm{H}), 0.89-0.86(\mathrm{~m}, 6 \mathrm{H})$.

${ }^{13}$ C NMR (100 MHz, CDCl3): $\delta$ 150.5, [142.7, 142.6], [142.0, 141.9], [134.5, 134.2], 131.3, [129.2, 129.0], 124.3, [121.7, 121.5], [121.04, 120.96], 108.5, 40.2, 39.7, [39.2, 39.1], [38.9, 38.6], 37.4, 36.1, [34.0, 33.5], [31.2, 30.9], [26.6, 26.5], 25.7, 20.7, [19.7, 19.3], 17.7, 17.4, [16.2, 16.1], 14.8 .

IR (neat, $\mathbf{c m}^{-1}$ ): 2966, 2931, 1726, 1666, 1451, 1377, 1051, 846.

HRMS (EI): $[\mathrm{M}]^{+}$calcd for $\mathrm{C}_{26} \mathrm{H}_{40} 352.3130$, found: 352.3127 . 


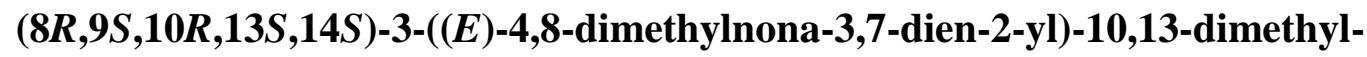
2,7,8,9,10,11,12,13,14,15,16,17-dodecahydro-1H-cyclopenta[a]phenanthren-17-yl acetate (8)

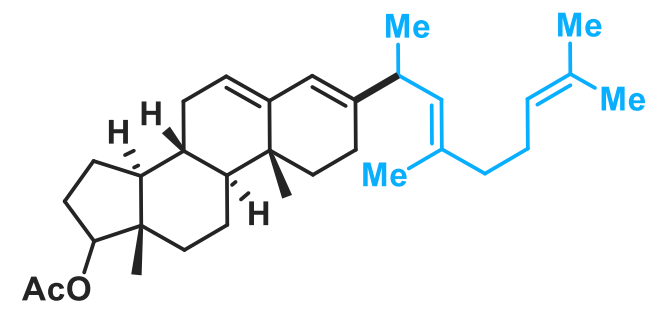

The target compound was prepared according to the General procedure at $25{ }^{\circ} \mathrm{C}$ from the reaction of $1 \mathrm{a}(30.0 \mathrm{mg}, 0.2 \mathrm{mmol})$ and 7 (166.4 mg, $0.36 \mathrm{mmol})$.

$42.7 \mathrm{mg}$, $46 \%$ yield, the regioisomeric ratio of $>20: 1$, white solid, $62-64{ }^{\circ} \mathrm{C}$.

${ }^{1}$ H NMR (400 MHz, CDCl $): \delta 5.74(\mathrm{~s}, 1 \mathrm{H}), 5.33(\mathrm{~d}, J=2.8 \mathrm{~Hz}, 1 \mathrm{H})$, 5.09-5.06 (m, $1 \mathrm{H}), 4.99(\mathrm{dd}, J=8.0,0.8 \mathrm{~Hz}, 1 \mathrm{H}), 4.61(\mathrm{t}, J=8.0 \mathrm{~Hz}, 1 \mathrm{H}), 3.02-2.94(\mathrm{~m}, 1 \mathrm{H}), 2.22-$ 2.13 (m, 3 H), 2.10-2.04 (m, 5 H), 2.00-1.93 (m, 3 H), 1.82-1.74 (m, 2 H), 1.71-1.69 (m, 2 H), 1.67 (s, 4 H), 1.63-1.60 (m, 7 H), 1.56-1.46 (m, 1 H), 1.44-1.29 (m, 2 H), 1.26-1.10 (m, $3 \mathrm{H}), 1.07$ (d, J = 6.8 Hz, $3 \mathrm{H}), 1.05-0.95(\mathrm{~m}, 1 \mathrm{H}), 0.91(\mathrm{~s}, 3 \mathrm{H}), 0.83$ (s, $3 \mathrm{H})$.

${ }^{13}$ C NMR (100 MHz, CDCl3): $\delta$ 171.2, 142.1, 141.3, 134.1, 131.3, 129.3, 124.3, 121.7, 120.9, 82.8, 51.3, 48.4, 42.5, 39.7, 39.0, 36.8, 35.1, 34.2, 31.7, 31.4, 27.6, 26.6, 25.7, $24.7,23.5,21.2,20.6,19.3,18.9,17.7,16.2,12.1$.

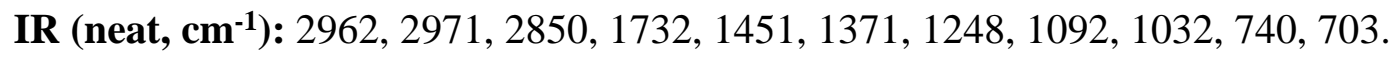
HRMS (ESI): [M+Na] ${ }^{+}$calcd for $\mathrm{C}_{32} \mathrm{H}_{48} \mathrm{NaO}_{2}$ 487.3547, found: 487.3547 .

$(8 R, 9 S, 10 R, 13 S, 14 S)-3-((S, 3 E, 5 E, 7 E, 9 E)-4,8-d i m e t h y l-10-(2,6,6-$ trimethylcyclohex-1-en-1-yl)deca-3,5,7,9-tetraen-2-yl)-10,13-dimethyl2,7,8,9,10,11,12,13,14,15,16,17-dodecahydro-1H-cyclopenta[a]phenanthren-17-yl acetate (10)

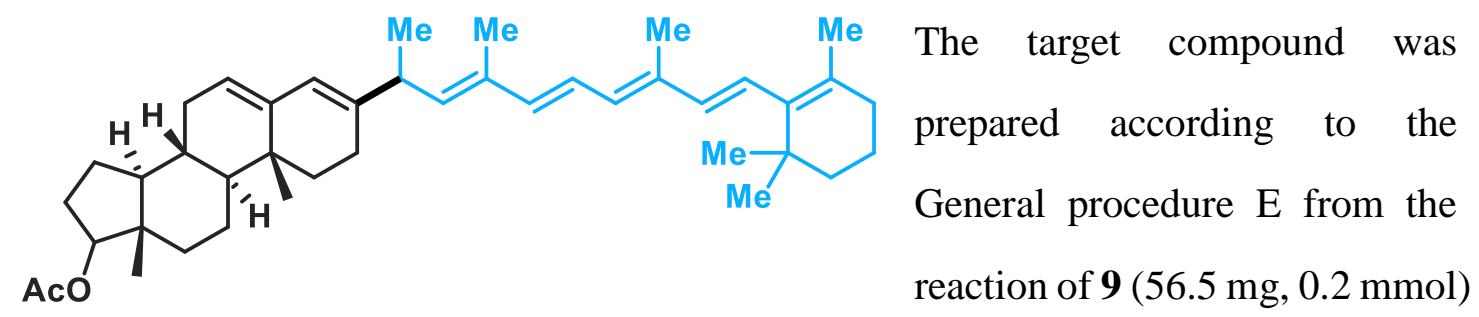
and 7 (166.4 mg, $0.36 \mathrm{mmol})$. 
$60.8 \mathrm{mg}, 51 \%$ yield, the regioisomeric ratio is $>20: 1, \mathrm{dr}=1.4: 1$, yellow oil. This compound is acid, air, and/or photo-sensitive, and it was purified by preparative HPLC. A few impurity was formed during the manipulation.

${ }^{1}$ H NMR (600 MHz, C6D6): $\delta$ 6.68-6.63 (m, 1 H), 6.41-6.23 (m, 4 H), 5.97 (s, 1 H), $5.51(\mathrm{t}, J=6.0,1 \mathrm{H}), 5.43(\mathrm{t}, J=6.0,1 \mathrm{H}), 4.74-4.71(\mathrm{~m}, 1 \mathrm{H}), 3.20-3.14(\mathrm{~m}, 1 \mathrm{H})$, 2.21-2.13 (m, 2 H), 2.08-2.03 (m, 2 H), 1.98-1.94 (m, 2 H), 1.92-1.89 (m, 3 H), 1.851.82 (m, 4 H), 1.81-1.79 (m, 3 H), 1.74-1.73 (m, 4 H), 1.61-1.56 (m, 5 H), 1.51-1.48 (m, 4 H), 1.41-1.36 (m, 1 H), 1.35-1.29 (m, 2 H), 1.23-1.20 (m, 1 H), 1.18-1.17 (m, 2 H), 1.13-1.11 (m, 7 H), 1.01-0.97 (m, 4 H), 0.83-0.81 (m, 4 H).

${ }^{13}$ C NMR (150 MHz, CDCl $)$ ): $\delta$ 170.1, 142.2, [139.93, 139.85], 138.8, [138.43, 138.41], [137.8, 137.6], [135.1, 135.0], [134.4, 134.2], 131.6, 129.1, 128.2, 126.3, [123.9, 123.8], 123.7, [122.0, 121.9], 82.8, [51.29, 51.26], 48.7, 42.8, 40.0, 37.2, [35.44, 35.42], [34.61, 34.58], 33.4, 32.0, 31.7, 30.3, 29.2, 28.0, 25.2, 24.9, 23.7, 22.0, 21.1, 20.8, [19.82, 19.79], 19.5, [19.24, 19.22], 12.9, [12.86, 12.84], 12.4, [12.36, 12.34].

IR (neat, $\mathbf{c m}^{-1}$ ): 2920, 2853, 1739, 1448, 1374, 1245, 1033, 967, 732.

HRMS (ESI): $[\mathrm{M}+\mathrm{H}]{ }^{+}$calcd for $\mathrm{C}_{42} \mathrm{H}_{61} \mathrm{O}_{2}$ 597.4666, found: 597.4678 . 


\section{Mechanistic Investigation}

\subsection{The reactivity of vinyl triflate and diene under the standard conditions.}

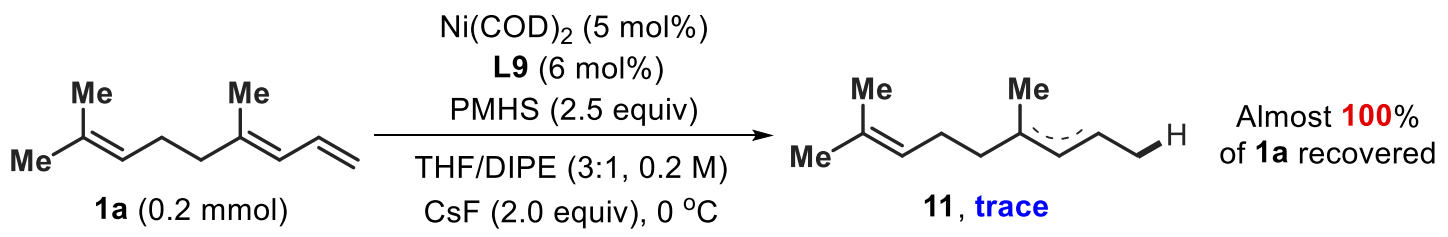

General procedure. The procedure was conducted in an argon-filled glove box. To a reaction tube equipped with a magnetic stir bar was charged with $\mathrm{Ni}(\mathrm{COD})_{2}(2.8 \mathrm{mg}, 5$ mol \%), L9 (6.1 mg, $6 \mathrm{~mol} \%), \mathrm{CsF}$ (60.8 mg, 2.0 equiv.), and THF/DIPE (1 mL, 3:1). PMHS (111.3 mg, $0.5 \mathrm{mmol})$ and diene $1 \mathbf{a}(30.0 \mathrm{mg}, 0.2 \mathrm{mmol})$ was added in sequence. The reaction tube was sealed and removed from the glove box. The reaction mixture was stirred at $0{ }^{\circ} \mathrm{C}$ for $48 \mathrm{~h}$. It was diluted with ethyl acetate $(10 \mathrm{~mL})$, washed with water and brine, and dried over anhydrous $\mathrm{Na}_{2} \mathrm{SO}_{4}$. A $0.2 \mathrm{~mL}$ of solution was collected, diluted with ethyl acetate $(2 \mathrm{~mL})$, and the result was analyzed by GC-MS.

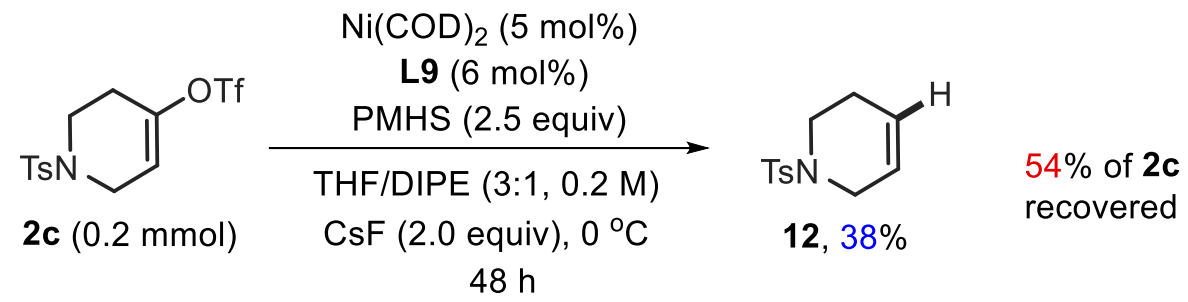

General procedure. The procedure was conducted in an argon-filled glove box. To a reaction tube equipped with a magnetic stir bar was charged with catalyst $\mathrm{Ni}(\mathrm{COD})_{2}$ (2.8 mg, $5 \mathrm{~mol} \%$ ), L9 (6.1 mg, $6 \mathrm{~mol} \%$ ), CsF (60.8 mg, 2.0 equiv.) and THF/DIPE (1 $\mathrm{mL}, 3: 1)$. PMHS (111.3 mg, $0.5 \mathrm{mmol})$ and vinyl triflate $2 \mathrm{c}(77.0 \mathrm{mg}, 0.2 \mathrm{mmol})$ were then added in sequence. The reaction tube was sealed and removed from the glove box. The reaction mixture was stirred at $0{ }^{\circ} \mathrm{C}$ for $48 \mathrm{~h}$. The solvent was removed, and the residue was purified by flash chromatography on silica gel to afford $\mathbf{1 2}$ in $38 \%$ yield $(18.0 \mathrm{mg})$ with $54 \%$ of $\mathbf{2 c}$ recovered.

${ }^{1} \mathrm{H}$ NMR and ${ }^{13} \mathrm{C}$ NMR data of $\mathbf{1 2}$ are consistent with those reported in reference. ${ }^{28}$

${ }^{1}$ H NMR (400 MHz, CDCl $): \delta 7.68(\mathrm{~d}, J=8.0 \mathrm{~Hz}, 2 \mathrm{H}), 7.32(\mathrm{~d}, J=8.0 \mathrm{~Hz}, 2 \mathrm{H})$, 
5.77-5.74 (m, $1 \mathrm{H})$, 5.63-5.60 (m, $1 \mathrm{H}), 3.58-3.57(\mathrm{~m}, 2 \mathrm{H}), 3.17(\mathrm{t}, J=5.6 \mathrm{~Hz}, 2 \mathrm{H})$, $2.43(\mathrm{~s}, 3 \mathrm{H}), 2.22(\mathrm{~m}, 2 \mathrm{H})$.

${ }^{13}$ C NMR (100 MHz, CDCl3): $\delta$ 143.5, 133.3, 129.6, 127.7, 125.0, 122.7, 44.8, 42.6, 25.3, 21.5 .

\subsection{The reaction of phenyl substituted 1,3-diene with $\mathrm{Si}-\mathrm{H}$}

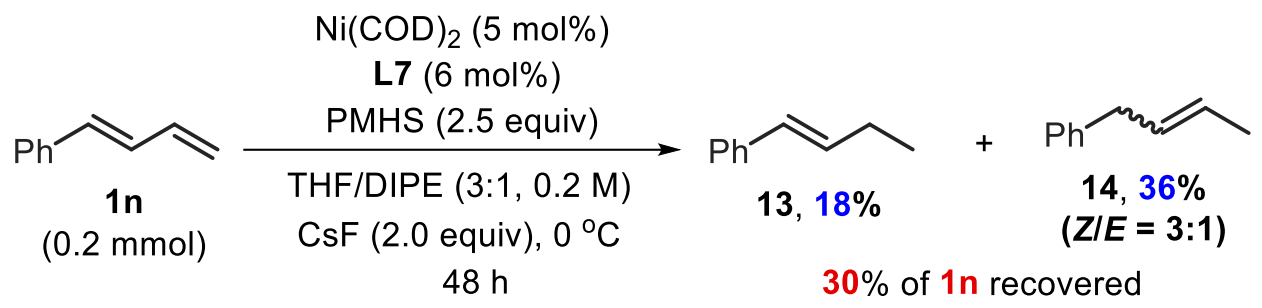

General procedure. The procedure was conducted in an argon-filled glove box. To a reaction tube equipped with a magnetic stir bar was charged with $\mathrm{Ni}(\mathrm{COD})_{2}(2.8 \mathrm{mg}, 5$ mol \%), L7 (3.4 mg, 6 mol \%), CsF (60.8 mg, 2.0 equiv.) and THF/DIPE (1 mL, 3:1). PMHS (111.3 mg, $0.5 \mathrm{mmol})$ and diene $1 \mathrm{n}(26.0 \mathrm{mg}, 0.2 \mathrm{mmol})$ were then added in sequence. The reaction tube was sealed and removed from the glove box. The reaction mixture was stirred at $0{ }^{\circ} \mathrm{C}$ for $48 \mathrm{~h}$. It was diluted with ethyl acetate $(10 \mathrm{~mL})$, washed with water and brine, dried over anhydrous $\mathrm{Na}_{2} \mathrm{SO}_{4}$, and concentrated. The yields were determined by ${ }^{1} \mathrm{H}$ NMR analysis versus the internal standard $\left(\mathrm{CH}_{2} \mathrm{Br}_{2}\right)$.

\subsection{Reactions to exclude the pathway via diene insertion into vinyl-Ni(II)-OTf}
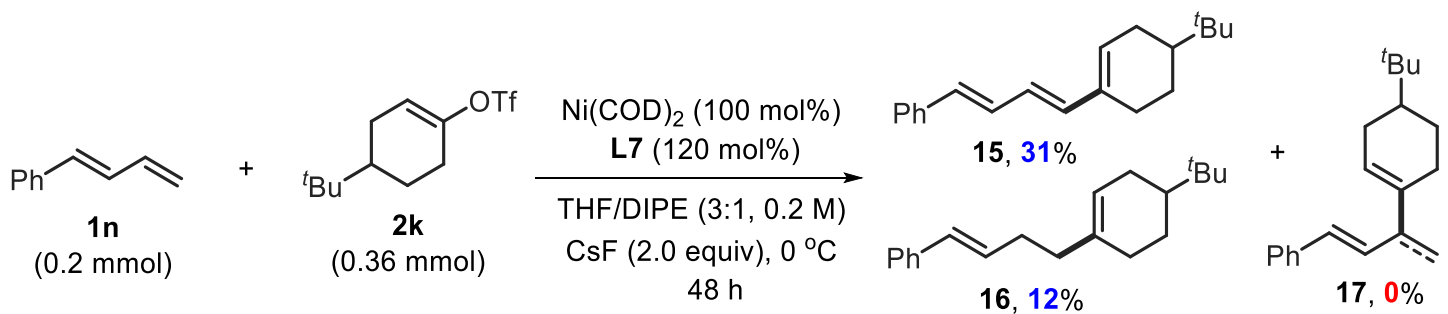

General procedure. The procedure was conducted in an argon-filled glove box. To a reaction tube equipped with a magnetic stir bar was charged with $\mathrm{Ni}(\mathrm{COD})_{2}(55.0 \mathrm{mg}$, $100 \mathrm{~mol} \%)$, L7 (68.9 mg, $120 \mathrm{~mol} \%), \mathrm{CsF}$ (60.8 mg, 2.0 equiv.), and THF/DIPE (1 $\mathrm{mL}, 3: 1)$. Diene 1n (26.0 mg, $0.2 \mathrm{mmol})$ and vinyl triflates $2 \mathbf{k}(103.0 \mathrm{mg}, 0.36 \mathrm{mmol})$ were then added in sequence. The reaction tube was sealed and removed from the glove 
box. The reaction mixture was stirred at $0{ }^{\circ} \mathrm{C}$ for $48 \mathrm{~h}$. The reaction mixture was diluted with ethyl acetate $(10 \mathrm{~mL})$, washed with water, brine, and dried over anhydrous $\mathrm{Na}_{2} \mathrm{SO}_{4}$. A $0.2 \mathrm{~mL}$ of solution was collected, diluted with ethyl acetate $(2 \mathrm{~mL})$, and analyzed by GC-MS. The rest was concentrated, and the residue was purified by flash chromatography on silica gel to afford $\mathbf{1 5}$ in $31 \%$ yield as a white solid, mp: $36-38^{\circ} \mathrm{C}$. The yield of 16 was determined by ${ }^{1} \mathrm{H}$ NMR, since it mixed with inseparable diene $\mathbf{1 n}$. ((1E,3E)-4-(4-(tert-butyl)cyclohex-1-en-1-yl)buta-1,3-dien-1-yl)benzene (15)

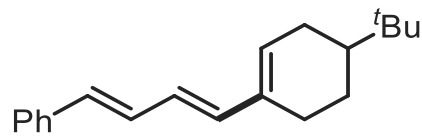

${ }^{1}$ H NMR (400 MHz, CDCl3): $\delta 7.39(\mathrm{~d}, J=8.0 \mathrm{~Hz}, 2 \mathrm{H}), 7.30(\mathrm{t}, J=8.0 \mathrm{~Hz}, 2 \mathrm{H})$, $7.19(\mathrm{t}, J=8.0 \mathrm{~Hz}, 1 \mathrm{H}), 6.83(\mathrm{dd}, J=16.0,10.0 \mathrm{~Hz}, 1 \mathrm{H}), 6.53(\mathrm{~d}, J=16.0 \mathrm{~Hz}, 1 \mathrm{H})$, 6.39-6.26 (m, 2 H), 5.84 (s, $1 \mathrm{H}), 2.42-2.37$ (m, $1 \mathrm{H}), 2.23-2.08$ (m, $2 \mathrm{H})$, 1.98-1.91 (m, $2 \mathrm{H}), 1.34-1.30$ (m, $1 \mathrm{H}), 1.22-1.18$ (m, $1 \mathrm{H}), 0.89$ (s, $9 \mathrm{H})$.

${ }^{13}$ C NMR (100 MHz, CDCl3): $\delta$ 137.7, 136.5, 135.9, 131.0, 130.95, 129.9, $128.6(2$ C), 127.1, 126.1 (2 C), 125.8, 44.2, 32.2, 27.8, 27.2 (3 C), 25.9, 23.8.

IR (neat, $\mathbf{c m}^{-1}$ ): 2961, 2922, 2872, 2363, 2343, 1366, 986, 911, 747, 691.

GC-MS (EI): $[\mathrm{M}]^{+}$calcd for $\mathrm{C}_{20} \mathrm{H}_{26}$ 266.20, found: 266.20 .

\section{Scheme S1. Effect of PMHS on the stoichiometric reaction of $1 \mathrm{a}$ and $2 \mathrm{w}$ with $\mathrm{Ni}(0)^{a}$}

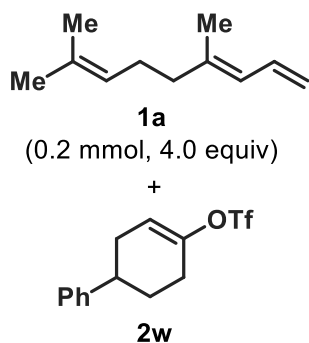

(0.2 mmol, 4.0 equiv)

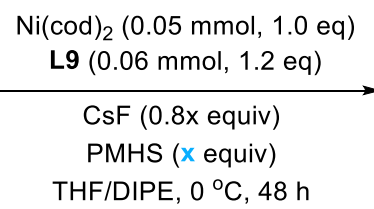
THF/DIPE, $0{ }^{\circ} \mathrm{C}, 48 \mathrm{~h}$
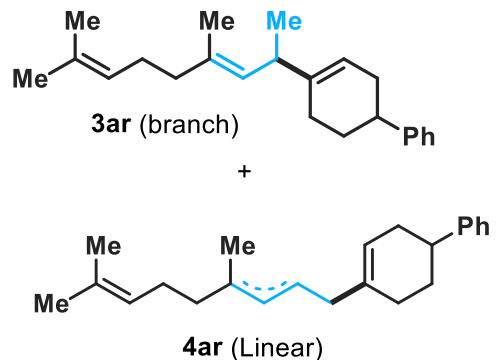

\begin{tabular}{cccc} 
Entry & PMHS (x equiv) & 3ar & 3ar/4ar \\
\hline $1^{b}$ & 1.0 & $20 \%$ & $1: 2$ \\
2 & 5.0 & $48 \%$ & $3: 1$ \\
3 & 12.0 & $72 \%$ & $11: 1$
\end{tabular}

${ }^{a}$ Yield with respect to the amount of $1 \mathbf{a} .{ }^{b}$ Yield with respect to the amount of PMHS. 
General Procedure. The procedure was conducted in an argon-filled glove box. To a reaction tube equipped with a magnetic stir bar was charged with $\mathrm{Ni}(\mathrm{COD})_{2}(14 \mathrm{mg}$, $0.05 \mathrm{mmol}, 1.0$ equiv), $\mathbf{L 9}$ (31 mg, $0.06 \mathrm{mmol}$ ), CsF ( $0.8 \mathrm{x}$ equiv), THF/DIPE (1.0 mL, 3: 1, $0.2 \mathrm{M})$, PMHS (x equiv), dienes $1 \mathbf{a}(30 \mathrm{mg}, 0.2 \mathrm{mmol})$, and vinyl triflates $\mathbf{2 w}$ (61 $\mathrm{mg}, 0.2 \mathrm{mmol})$. The reaction tube was sealed and removed from the glove box. The reaction mixture was stirred at $0{ }^{\circ} \mathrm{C}$ for $48 \mathrm{~h}$. The reaction mixture was diluted with ethyl acetate $(10 \mathrm{~mL})$, washed with water, brine, and dried over anhydrous $\mathrm{Na}_{2} \mathrm{SO}_{4}$. A $0.2 \mathrm{~mL}$ of solution was collected and diluted with ethyl acetate $(2 \mathrm{~mL})$. The yield and the ratio of 3ar/4ar were determined by GC analysis versus the internal standard (Dodecane).

4-((R,E)-4,8-dimethylnona-3,7-dien-2-yl)-1,2,3,6-tetrahydro-1,1'-biphenyl (3ar)<smiles>CC(C)=CCC/C(C)=C/C(C)C1=CCC(c2ccccc2)CC1</smiles>

${ }^{1} \mathbf{H}$ NMR (400 MHz, CDCl3, dr = 1.7:1): $\delta$ 7.35-7.32 (m, $\left.2 \mathrm{H}\right), 7.28-7.21(\mathrm{~m}, 3 \mathrm{H})$, $5.56(\mathrm{~d}, J=2.8 \mathrm{~Hz}, 1 \mathrm{H}), 5.15-5.12(\mathrm{~m}, 1 \mathrm{H}), 5.05(\mathrm{~d}, J=8.0 \mathrm{~Hz}, 1 \mathrm{H}), 3.04-2.95(\mathrm{~m}, 1$ H), 2.81-2.75 (m, $1 \mathrm{H}), 2.36-2.32(\mathrm{~m}, 1 \mathrm{H}), 2.22-2.11(\mathrm{~m}, 5 \mathrm{H}), 2.06(\mathrm{~d}, J=8.0 \mathrm{~Hz}, 2$ H), 2.00-1.97 (m, 1 H), 1.72 (m, 4 H), 1.69-1.67 (m, 3 H), 1.65 (s, 3 H), 1.11 (d, J = 6.8 $\mathrm{Hz}, 3 \mathrm{H})$.

${ }^{13}$ C NMR (100 MHz, $\mathbf{C D C l}_{3}, \mathbf{d r}=\mathbf{1 . 7 : 1 ) :} \delta$ [147.5, 147.4], [142.0, 141.8], [134.1, 133.9], 131.3, [129.6, 129.5], 128.3, 126.9, 125.9, 124.4, [118.6, 118.5], [40.5, 40.4], $[39.8,39.75],[39.2,38.9],[33.8,33.6],[30.3,30.2],[27.4,27.3], 26.6,25.7,[19.6$, 19.4], 17.7, [16.13, 16.1.].

IR (neat, $\left.\mathbf{c m}^{-1}\right)$ : 2965, 2929, 1485, 1449, 1377, 822, 765, 735 .

GC-MS (EI): [M] ${ }^{+}$calcd for $\mathrm{C}_{21} \mathrm{H}_{36}$ 308.25, found: 308.16 . 


\subsection{Reactions that is consistent with the intermediacy of $\pi$-allylnickel species}

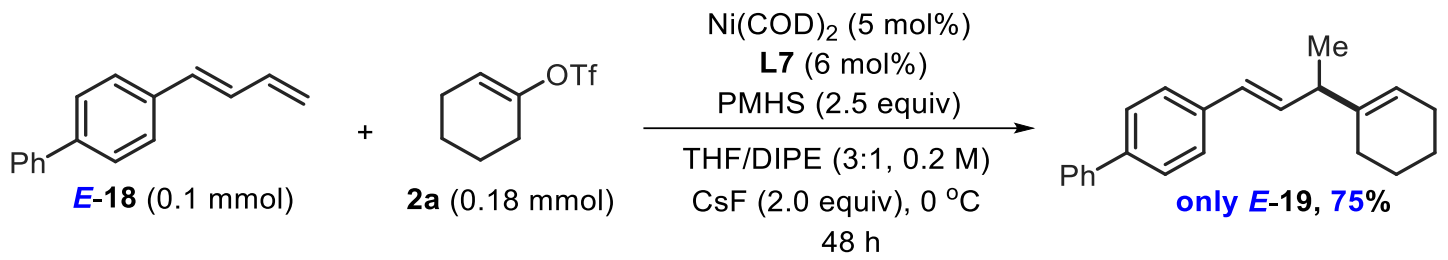

General Procedure. The procedure was conducted in an argon-filled glove box. To a reaction tube equipped with a magnetic stir bar was charged with $\mathrm{Ni}(\mathrm{COD})_{2}(1.4 \mathrm{mg}$, $0.005 \mathrm{mmol})$, $\mathbf{L} 7$ (1.7 mg, $0.006 \mathrm{mmol}), \mathrm{CsF}$ (30.4 mg, $0.2 \mathrm{mmol})$, and THF/DIPE (0.5 mL, 3: 1, 0.2 M). PMHS (55.7 mg, $0.25 \mathrm{mmol})$, dienes $\boldsymbol{E}-18$ (20.6 mg, $0.1 \mathrm{mmol})$ and vinyl triflate $2 \mathbf{a}(41.4 \mathrm{mg}, 0.18 \mathrm{mmol})$ were then added in sequence. The reaction tube was sealed and removed from the glove box. The reaction mixture was stirred at $0{ }^{\circ} \mathrm{C}$ for $48 \mathrm{~h}$. The solvent was removed, and the residue was purified by flash chromatography on silica gel to afford product $\boldsymbol{E}-19$.

$21.6 \mathrm{mg}, 75 \%$ yield, the regioisomeric ratio of $>20: 1$, white solid, $\mathrm{mp}: 42-45^{\circ} \mathrm{C}$.

${ }^{1}$ H NMR (400 MHz, CDCl3): $\delta 7.59(\mathrm{~d}, J=8.0 \mathrm{~Hz}, 2 \mathrm{H}), 7.54(\mathrm{~d}, J=8.0 \mathrm{~Hz}, 2 \mathrm{H})$, 7.45-7.41 (m, $4 \mathrm{H}), 7.33$ (t, $J=8.0 \mathrm{~Hz}, 1 \mathrm{H}), 6.39$ (d, $J=16.0 \mathrm{~Hz}, 1 \mathrm{H}), 6.21(\mathrm{dd}, J=$ 16.0, $8.0 \mathrm{~Hz}, 1 \mathrm{H}), 5.53(\mathrm{~s}, 1 \mathrm{H}), 2.91-2.85$ (m, $1 \mathrm{H}), 2.04-1.92(\mathrm{~m}, 4 \mathrm{H}), 1.65-1.55$ (m, $4 \mathrm{H}), 1.21(\mathrm{~d}, J=7.2 \mathrm{~Hz}, 3 \mathrm{H})$.

${ }^{13}$ C NMR (100 MHz, CDCl3): $\delta 140.9$ (2 C), 139.6, 136.9, 135.4, 128.7, 127.9, 127.2, $127.1,126.9,126.4,120.7,44.3,26.6,25.3,23.1,22.7,18.5$.

IR (neat, $\mathbf{c m}^{-1}$ ): 2928, 2855, 2365, 2342, 1657, 1638, 1487, 848, 766, 749, 697.

HRMS (EI): [M] ${ }^{+}$calcd for $\mathrm{C}_{22} \mathrm{H}_{24} 288.1878$, found: 288.1872 .
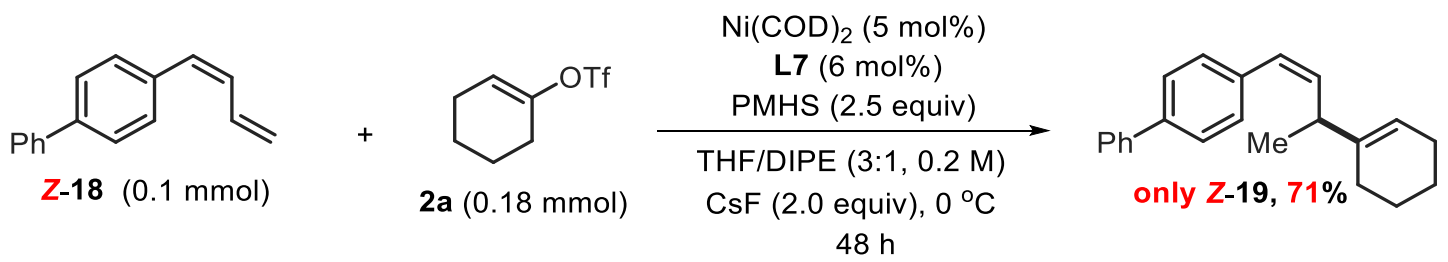

General Procedure. The procedure was conducted in an argon-filled glove box. To a reaction tube equipped with a magnetic stir bar was charged with $\mathrm{Ni}(\mathrm{COD})_{2}(1.4 \mathrm{mg}$, $0.005 \mathrm{mmol}$ ), $\mathbf{L} 7$ (1.7 mg, $0.006 \mathrm{mmol}), \mathrm{CsF}$ (30.4 mg, $0.2 \mathrm{mmol}$ ), and THF/DIPE (0.5 mL, 3: 1, 0.2 M). PMHS (55.7 mg, $0.25 \mathrm{mmol})$, dienes $\boldsymbol{Z}-18$ (20.6 mg, $0.1 \mathrm{mmol})$ and 
vinyl triflates $2 \mathbf{a}(41.4 \mathrm{mg}, 0.18 \mathrm{mmol})$ were then added in sequence. The reaction tube was sealed and removed from the glove box. The reaction mixture was stirred at $0{ }^{\circ} \mathrm{C}$ for $48 \mathrm{~h}$. The solvent was removed, and the residue was purified by flash chromatography on silica gel to afford product $Z \mathbf{Z - 1 9}$.

$20.5 \mathrm{mg}, 71 \%$ yield, the regioisomeric ratio of $>20: 1$, colorless oil.

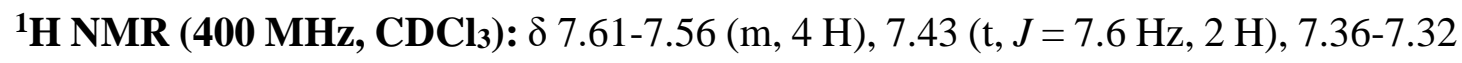
$(\mathrm{m}, 3 \mathrm{H}), 6.44(\mathrm{~d}, J=12.0 \mathrm{~Hz}, 1 \mathrm{H}), 5.61(\mathrm{t}, J=12.0 \mathrm{~Hz}, 1 \mathrm{H}), 5.55(\mathrm{~s}, 1 \mathrm{H}), 3.35-3.28$ (m, $1 \mathrm{H}), 2.02-1.99(\mathrm{~m}, 4 \mathrm{H}), 1.66-1.54(\mathrm{~m}, 4 \mathrm{H}), 1.15(\mathrm{~d}, J=6.8 \mathrm{~Hz}, 3 \mathrm{H})$.

${ }^{13}$ C NMR (100 MHz, CDCl3): $\delta$ 141.2, 140.9, 139.2, 137.6, 136.8, 129.2, 128.7, 127.3, $127.2,127.0,126.8,120.3,39.5,26.8,25.3,23.1,22.7,20.0$.

IR (neat, $\mathbf{c m}^{-1}$ ): 2922, 1640, 971, 822, 786, 688.

HRMS (EI): [M] ${ }^{+}$calcd for $\mathrm{C}_{22} \mathrm{H}_{24} 288.1878$, found: 288.1875 .

\section{Scheme S2. Stereochemistry of aliphatic dienes}

(a)
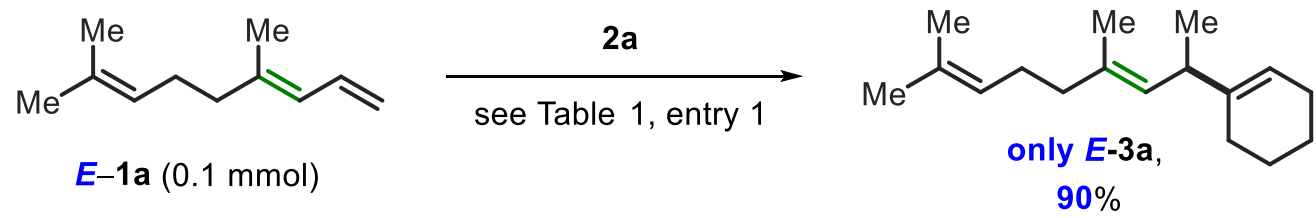

(b)
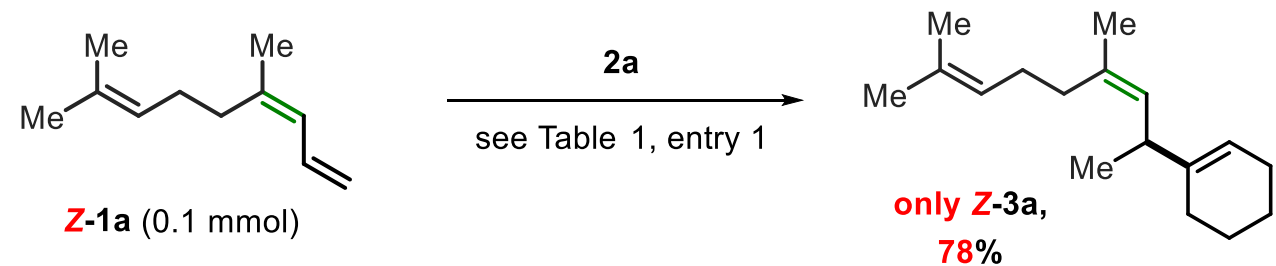

General Procedure. The procedure was conducted in an argon-filled glove box. To a reaction tube equipped with a magnetic stir bar was charged with $\mathrm{Ni}(\mathrm{COD})_{2}(1.4 \mathrm{mg}$, $0.005 \mathrm{mmol}), \mathbf{L 9}$ (3.1 mg, $0.006 \mathrm{mmol}), \mathrm{CsF}$ (30.4 mg, $0.2 \mathrm{mmol})$, and THF/DIPE (0.5 mL, 3: 1, 0.2 M). PMHS (55.7 mg, $0.25 \mathrm{mmol})$, dienes $E-1 \mathbf{a}$ or Z-1a $(15.0 \mathrm{mg}, 0.1$ mmol) and vinyl triflates $\mathbf{2 a}(41.4 \mathrm{mg}, 0.18 \mathrm{mmol})$ were then added in sequence. The reaction tube was sealed and removed from the glove box. The reaction mixture was 
stirred at $0{ }^{\circ} \mathrm{C}$ for $48 \mathrm{~h}$. The solvent was removed in vacuum, and the residue was purified by flash chromatography on silica gel to afford product $E-3 \mathbf{a}$ ( $20.9 \mathrm{mg}, 90 \%$ yield, regioisomeric ratio of >20:1) or Z-3a $(18.1 \mathrm{mg}, 78 \%$ yield, the regioisomeric ratio of $>20: 1)$.

${ }^{1} \mathrm{H}$ NMR (400 MHz, CDCl3, Z-3a): $\delta 5.41(\mathrm{~s}, 1 \mathrm{H}), 5.12(\mathrm{~s}, 1 \mathrm{H}), 5.01(\mathrm{~d}, J=9.6 \mathrm{~Hz}$, $1 \mathrm{H}), 2.94-2.88(\mathrm{~m}, 1 \mathrm{H}), 2.05-1.90(\mathrm{~m}, 8 \mathrm{H}), 1.69(\mathrm{~m}, 6 \mathrm{H}), 1.68(\mathrm{~s}, 6 \mathrm{H}), 1.61-1.53(\mathrm{~m}$, $7 \mathrm{H}), 1.02(\mathrm{~d}, J=6.8 \mathrm{~Hz}, 3 \mathrm{H})$.

${ }^{13}$ C NMR (100 MHz, CDCl3, Z-3a): $\delta$ 142.1, 133.9, 131.4, 130.2, 124.5,119.0, 39.3, $32.1,26.7,26.65,25.7,25.3,23.4,23.3,22.8,19.9,17.6$.

IR (neat, $\mathbf{c m}^{-1}$ ): 2983, 2926, 1662, 1455, 1377, 1281, 1097, 918, 723.

GC-MS (EI): $[\mathrm{M}]^{+}$calcd for $\mathrm{C}_{17} \mathrm{H}_{28}$ 232.22, found: 232.23 .

\subsection{Monitoring of the reactions catalyzed by $\mathrm{Ni}(0)$ and $\mathrm{Ni}(\mathrm{II})$}

General Procedure. The procedure was conducted in an argon-filled glove box. To a reaction tube equipped with a magnetic stir bar was charged with nickel catalyst (5 mol\%, $0.02 \mathrm{mmol}), \mathbf{L} 7$ (6.8 mg, $6 \mathrm{~mol} \%, 0.024 \mathrm{mmol}), \mathrm{CsF}$ (121.6 mg, $0.8 \mathrm{mmol})$, and THF/DIPE (2.0 mL, 3:1, 0.2 M). PMHS (222.6 mg, 1.0 mmol), dienes $1 \mathbf{n}(52.0 \mathrm{mg}, 0.4$ mmol) and vinyl triflate $\mathbf{2 a}(166.0 \mathrm{mg}, 0.72 \mathrm{mmol})$ were then added in sequence. The reaction mixture was stirred at room temperature. A $30 \mu \mathrm{L}$ of solution was collected in every 5 to $60 \mathrm{~min}$. It was quenched with $50 \mu \mathrm{L}$ of $\mathrm{H}_{2} \mathrm{O}$, diluted with ethyl acetate (1 $\mathrm{mL}$ ), and filtered through a syringe filter. The filtrate was analyzed by GC analysis and the yield was calculated versus the internal standard (dodecane). 
Scheme S3. Monitoring of the reactions catalyzed by $\mathrm{Ni}(0)$ and $\mathrm{Ni}(\mathrm{II})$
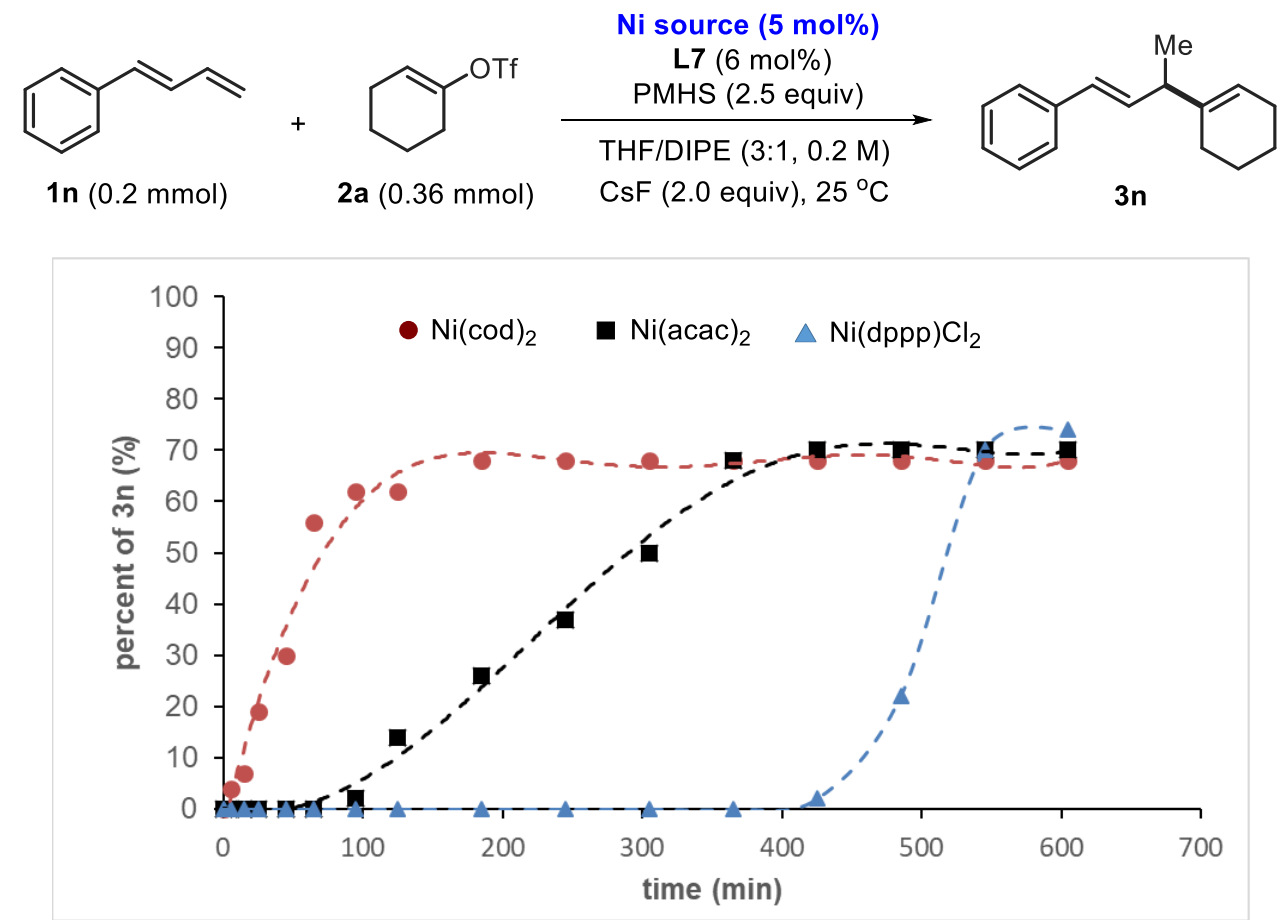

\subsection{An alternative mechanism}

\section{Scheme S4. Proposed alternative mechanism}

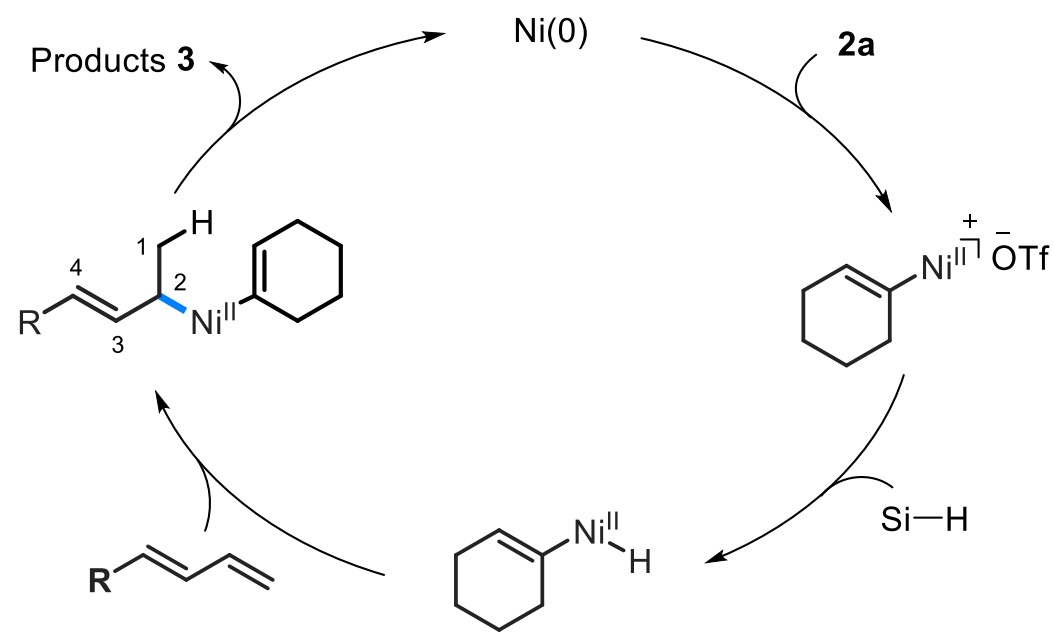




\section{Synthesis and Crystallographic Data of Complex A}

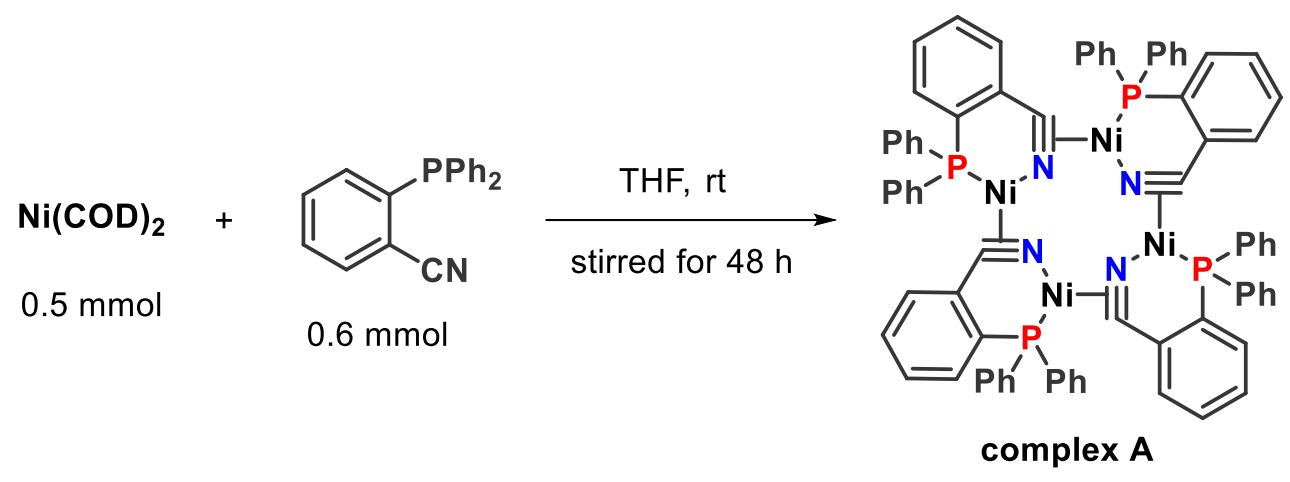

General Procedure. The procedure was conducted in an argon-filled glove box. To a reaction tube equipped with a magnetic stir bar was charged with $\mathrm{Ni}(\mathrm{COD})_{2}(137 \mathrm{mg}$, $0.5 \mathrm{mmol})$, L7 (172 mg, $0.6 \mathrm{mmol})$, and THF (2.5 mL). The reaction mixture was stirred at room temperature for $48 \mathrm{~h}$, and the solvent was then removed in vacuum. The residue was washed with hexane for three times and dried in vacuum for $2 \mathrm{~h}$. It was purified by recrystallization from DMA-hexane to afford complex A (121 $\mathrm{mg}, 67 \%$ yield) as a black crystal.

${ }^{1}$ H NMR (400 MHz, THF-d8): $\delta 7.64(\mathrm{ddd}, J=7.2,7.2,1.2 \mathrm{~Hz}, 16 \mathrm{H}) ; 7.37-7.29(\mathrm{~m}$, $24 \mathrm{H}) ; 7.13$ (ddd, $J=7.6,7.6,1.2 \mathrm{~Hz}, 4 \mathrm{H}) ; 7.04$ (ddd, $J=7.2,7.2,1.2 \mathrm{~Hz}, 4 \mathrm{H}) ; 6.92$ (dd, $J=7.6,7.6 \mathrm{~Hz}, 4 \mathrm{H}) ; 6.50$ (dd, $J=7.6,2.4 \mathrm{~Hz}, 4 \mathrm{H})$.

${ }^{31}$ P NMR (161 MHz, THF-d8): $\delta 35.4$. 


\section{Crystallographic Data of Complex A}

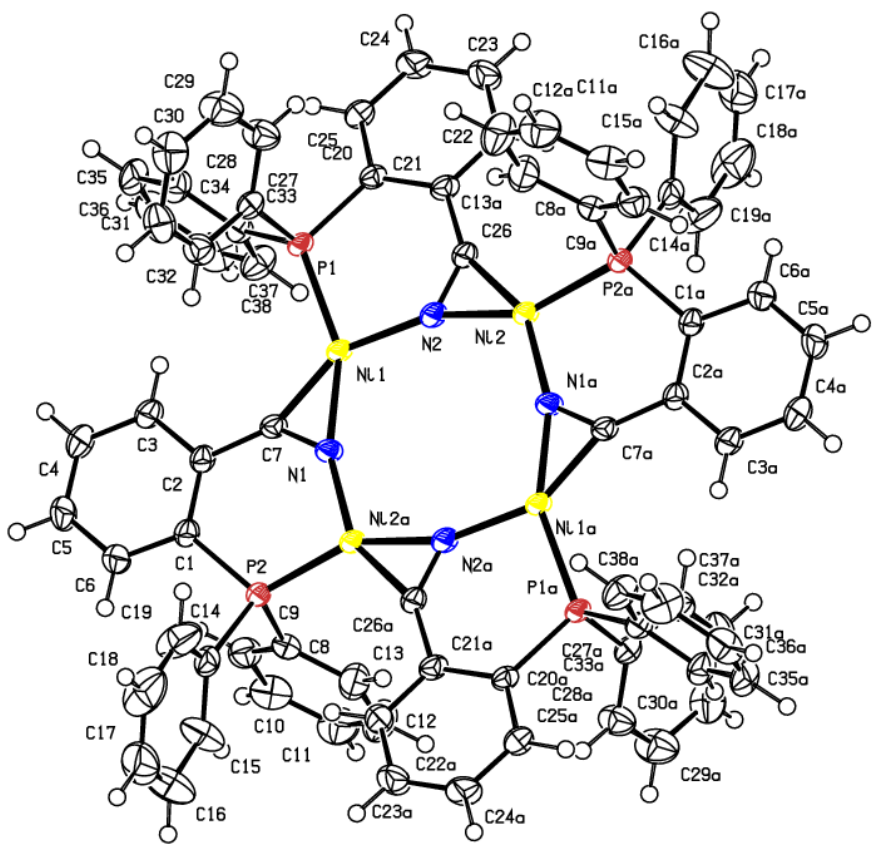

shuxz_1105_3

Table 1 Crystal data and structure refinement for shuxz_1105_3.

Identification code

Empirical formula

Formula weight

Temperature/K

Crystal system

Space group

$\mathrm{a} / \AA$

$\mathrm{b} / \AA$

$\mathrm{c} / \AA$

$\alpha /{ }^{\circ}$

$\beta /{ }^{\circ}$ shuxz_1105_3

$\mathrm{C}_{84} \mathrm{H}_{74} \mathrm{~N}_{6} \mathrm{Ni}_{4} \mathrm{O}_{2} \mathrm{P}_{4}$

1558.21

173.20(10)

triclinic

P-1

14.4274(4)

15.0616(5)

22.4243(6)

92.942(2)

101.454(2) 
Volume $/ \AA^{3}$

Z

$\rho_{\text {calc } g / \mathrm{cm}^{3}}$

$\mu / \mathrm{mm}^{-1}$

$\mathrm{F}(000)$

Crystal size $/ \mathrm{mm}^{3}$

Radiation

$2 \Theta$ range for data collection $/{ }^{\circ} 6.992$ to 133.2

Index ranges

$-13 \leq \mathrm{h} \leq 17,-17 \leq \mathrm{k} \leq 17,-26 \leq 1 \leq 26$

Reflections collected 29377

Independent reflections $15043\left[\mathrm{R}_{\text {int }}=0.0212, \mathrm{R}_{\text {sigma }}=0.0298\right]$

Data/restraints/parameters

Goodness-of-fit on $\mathrm{F}^{2}$

Final $R$ indexes $[\mathrm{I}>=2 \sigma(\mathrm{I})] \quad \mathrm{R}_{1}=0.0361, \mathrm{wR}_{2}=0.0970$

Final $\mathrm{R}$ indexes [all data] $\quad \mathrm{R}_{1}=0.0414, \mathrm{wR}_{2}=0.1011$

Largest diff. peak/hole / e $\AA^{-3} 1.08 /-0.50$

Table 2 Fractional Atomic Coordinates $\left(\times 10^{4}\right)$ and Equivalent Isotropic Displacement Parameters $\left(\AA^{2} \times 10^{3}\right)$ for shuxz_1105_3. Ueq is defined as $1 / 3$ of of the trace of the orthogonalised $\mathrm{U}_{\mathrm{IJ}}$ tensor.

\begin{tabular}{lll}
\multicolumn{2}{l}{ Atom $\boldsymbol{x}$} & $\boldsymbol{y}$ \\
$\mathrm{Ni} 1$ & $9473.2(3)$ & $388.4(3)$ \\
$\mathrm{Ni} 2$ & $10155.7(3)$ & $-1536.4(3)$ \\
$\mathrm{P} 1$ & $9356.1(4)$ & $1475.6(4)$ \\
$\mathrm{P} 2$ & $9818.7(4)$ & $-2574.8(4)$ \\
$\mathrm{N} 1$ & $9735.5(15)$ & $-710.1(13)$ \\
$\mathrm{N} 2$ & $9535.6(15)$ & $1117.7(13)$
\end{tabular}
$z$

$\mathbf{U}(\mathbf{e q})$

$1008.1(2)$

22.88(9)

409.1(2)

24.19(9)

1596.2(2)

21.06(11)

$1042.5(2)$

23.56(12)

836.1(8)

26.0(4)

340.1(8)

26.8(4) 


\begin{tabular}{|c|c|c|c|c|}
\hline $\mathrm{C} 1$ & $9677.4(17)$ & $-2123.5(16)$ & 1779.6(9) & $25.5(4)$ \\
\hline $\mathrm{C} 2$ & $9512.0(16)$ & $-1273.0(16)$ & $1841.5(9)$ & $23.9(4)$ \\
\hline $\mathrm{C} 3$ & $9331.5(18)$ & $-978.2(17)$ & $2392.0(10)$ & $29.4(5)$ \\
\hline $\mathrm{C} 4$ & $9330(2)$ & $-1508.7(19)$ & $2877.2(11)$ & $37.0(6)$ \\
\hline $\mathrm{C} 5$ & $9522(2)$ & $-2328(2)$ & $2828.3(11)$ & $38.9(6)$ \\
\hline C6 & $9692(2)$ & $-2634.7(18)$ & $2281.0(11)$ & $33.9(5)$ \\
\hline $\mathrm{C} 7$ & $9544.9(16)$ & $-665.8(15)$ & $1348.9(9)$ & $23.3(4)$ \\
\hline $\mathrm{C} 8$ & $8521.2(18)$ & $-3630.1(16)$ & $710.5(10)$ & $28.4(5)$ \\
\hline $\mathrm{C} 9$ & $7790.5(19)$ & $-4124.9(18)$ & $1043.2(12)$ & $36.0(5)$ \\
\hline $\mathrm{C} 10$ & $6815(2)$ & $-4908(2)$ & 751.1(14) & $45.2(6)$ \\
\hline $\mathrm{C} 11$ & $6565(2)$ & $-5203(2)$ & $128.1(14)$ & $50.9(7)$ \\
\hline $\mathrm{C} 12$ & $7292(2)$ & $-4721(2)$ & $-209.9(14)$ & $54.3(8)$ \\
\hline $\mathrm{C} 13$ & $8263(2)$ & $-3941(2)$ & $76.7(12)$ & $42.4(6)$ \\
\hline $\mathrm{C} 14$ & 10687.7(19) & $-3159.5(18)$ & $1270.9(10)$ & $31.9(5)$ \\
\hline $\mathrm{C} 15$ & $10347(3)$ & $-4171(2)$ & $1133.4(16)$ & $52.6(7)$ \\
\hline $\mathrm{C} 16$ & $11050(3)$ & $-4575(3)$ & $1255.4(19)$ & $69.8(10)$ \\
\hline $\mathrm{C} 17$ & $12073(3)$ & $-3992(3)$ & $1525.5(15)$ & $64.8(9)$ \\
\hline $\mathrm{C} 18$ & $12437(3)$ & $-2990(3)$ & $1663.0(16)$ & $71.9(11)$ \\
\hline C19 & $11736(2)$ & $-2570(3)$ & $1531.0(15)$ & $60.0(9)$ \\
\hline $\mathrm{C} 20$ & $8918.5(16)$ & $2308.1(15)$ & 1188.0(9) & $22.9(4)$ \\
\hline $\mathrm{C} 21$ & 9008.9(17) & $2430.8(16)$ & $579.9(10)$ & $25.4(4)$ \\
\hline $\mathrm{C} 22$ & $8661(2)$ & $3057.6(18)$ & $277.0(11)$ & $34.5(5)$ \\
\hline $\mathrm{C} 23$ & $8202(2)$ & $3542(2)$ & $558.5(12)$ & $41.5(6)$ \\
\hline $\mathrm{C} 24$ & $8109(2)$ & $3425(2)$ & $1154.9(12)$ & $40.4(6)$ \\
\hline $\mathrm{C} 25$ & $8468.9(19)$ & 2820.1(18) & $1464.7(11)$ & $31.8(5)$ \\
\hline $\mathrm{C} 26$ & $9393.8(17)$ & $1856.5(16)$ & $241.0(9)$ & $25.0(4)$ \\
\hline $\mathrm{C} 27$ & $10671.7(16)$ & $2322.7(16)$ & 2074.4(9) & $24.9(4)$ \\
\hline
\end{tabular}




\begin{tabular}{|c|c|c|c|c|}
\hline $\mathrm{C} 28$ & $11078(2)$ & $3347.5(19)$ & $2155.9(13)$ & $40.0(6)$ \\
\hline $\mathrm{C} 29$ & $12085(2)$ & $3943(2)$ & $2524.4(15)$ & $51.7(7)$ \\
\hline $\mathrm{C} 30$ & $12698(2)$ & $3522(2)$ & $2811.5(13)$ & $45.7(7)$ \\
\hline C31 & $12305(2)$ & $2505(2)$ & $2731.1(12)$ & $41.6(6)$ \\
\hline $\mathrm{C} 32$ & $11304.7(19)$ & 1906.3(19) & $2364.5(11)$ & $33.8(5)$ \\
\hline C33 & $8524.0(17)$ & 1126.1(16) & $2143.1(10)$ & $24.9(4)$ \\
\hline C34 & $8850.7(19)$ & $1581.3(19)$ & $2750.1(10)$ & $32.1(5)$ \\
\hline $\mathrm{C} 35$ & $8154(2)$ & $1280(2)$ & $3137.8(11)$ & $40.1(6)$ \\
\hline $\mathrm{C} 36$ & $7142(2)$ & $551(2)$ & 2916.3(13) & $42.1(6)$ \\
\hline C37 & $6805(2)$ & $101(2)$ & $2309.9(14)$ & $48.9(7)$ \\
\hline C38 & $7494(2)$ & $377.5(19)$ & $1928.2(12)$ & $39.7(6)$ \\
\hline $\mathrm{Ni3}$ & $5296.0(3)$ & $-4474.7(2)$ & $3927.7(2)$ & 19.17(8) \\
\hline $\mathrm{Ni} 4$ & $5315.3(3)$ & $-6558.2(2)$ & $4735.8(2)$ & 20.52(9) \\
\hline P3 & $5739.7(4)$ & $-3218.7(4)$ & $3447.7(2)$ & $20.43(11)$ \\
\hline P4 & $5480.6(4)$ & $-7503.4(4)$ & $4062.8(2)$ & $21.04(11)$ \\
\hline N3 & $5306.8(13)$ & $-5650.4(12)$ & $4177.0(8)$ & $21.7(4)$ \\
\hline N4 & $4857.0(14)$ & $-3827.9(13)$ & $4491.2(8)$ & $22.5(4)$ \\
\hline $\mathrm{C} 1 \mathrm{~A}$ & 6473(2) & $-5512(2)$ & 2857.1(13) & $45.1(7)$ \\
\hline C39 & $5627.8(16)$ & $-5471.5(15)$ & $3704.0(9)$ & $22.2(4)$ \\
\hline $\mathrm{C} 40$ & $6050.8(17)$ & $-5966.9(17)$ & $3331.6(10)$ & $27.7(5)$ \\
\hline $\mathrm{C} 41$ & 6057.1(17) & $-6872.2(16)$ & $3452.4(10)$ & $26.9(5)$ \\
\hline $\mathrm{C} 42$ & $6492(2)$ & $-7292(2)$ & $3087.5(12)$ & $41.7(6)$ \\
\hline $\mathrm{C} 43$ & $6894(3)$ & $-6840(3)$ & $2614.4(15)$ & $62.8(9)$ \\
\hline $\mathrm{C} 44$ & $6882(3)$ & $-5953(3)$ & $2497.8(16)$ & $66.1(10)$ \\
\hline $\mathrm{C} 45$ & $6270.1(17)$ & $-8148.3(16)$ & $4325.3(10)$ & $25.5(4)$ \\
\hline C46 & 7354.8(19) & $-7589(2)$ & $4563.6(12)$ & $39.4(6)$ \\
\hline $\mathrm{C} 47$ & $7964(2)$ & $-8036(2)$ & $4828.8(13)$ & $49.7(7)$ \\
\hline
\end{tabular}




\begin{tabular}{|c|c|c|c|c|}
\hline $\mathrm{C} 48$ & $7496(2)$ & $-9036(2)$ & $4870.2(13)$ & $49.0(7)$ \\
\hline C49 & $6433(2)$ & $-9588(2)$ & $4636.3(14)$ & $48.7(7)$ \\
\hline C50 & $5816(2)$ & $-9153.4(18)$ & $4362.5(12)$ & $35.8(5)$ \\
\hline C51 & 4207.7(17) & $-8511.2(16)$ & $3630.2(10)$ & $26.7(5)$ \\
\hline C52 & $3384.5(19)$ & $-8874.6(18)$ & $3922.9(12)$ & $36.9(5)$ \\
\hline C53 & $2405(2)$ & $-9644(2)$ & $3616.1(15)$ & $49.2(7)$ \\
\hline C54 & $2243(2)$ & $-10051(2)$ & $3019.9(15)$ & $50.6(8)$ \\
\hline C55 & $3045(2)$ & $-9697(2)$ & $2723.0(13)$ & $50.3(7)$ \\
\hline C56 & $4026(2)$ & $-8935.4(19)$ & $3023.3(11)$ & $38.1(6)$ \\
\hline C57 & $4567.7(16)$ & $-2494.9(15)$ & 4009.6(9) & $22.8(4)$ \\
\hline C58 & 5001.1(16) & $-2494.1(15)$ & 3497.1(9) & $21.9(4)$ \\
\hline C59 & $4832.6(18)$ & $-1947.2(17)$ & $3037.7(10)$ & $28.7(5)$ \\
\hline C60 & 4249.8(19) & $-1425.6(17)$ & $3079.2(11)$ & $32.8(5)$ \\
\hline C61 & 3823.9(19) & $-1438.5(18)$ & $3579.4(11)$ & $33.3(5)$ \\
\hline C62 & $3989.5(18)$ & $-1962.5(17)$ & $4046.0(10)$ & $29.2(5)$ \\
\hline C63 & $4678.1(16)$ & $-3093.4(15)$ & 4491.4(9) & $22.0(4)$ \\
\hline C64 & $7112.2(16)$ & $-2339.7(15)$ & $3830.7(9)$ & $22.4(4)$ \\
\hline C65 & $7471.2(18)$ & $-1318.1(17)$ & $3951.7(10)$ & $29.4(5)$ \\
\hline C66 & $8522.0(19)$ & $-702.0(18)$ & $4244.9(11)$ & $35.7(5)$ \\
\hline C67 & $9212.7(18)$ & $-1105.8(19)$ & $4420.0(10)$ & $34.4(5)$ \\
\hline C68 & $8872.5(18)$ & $-2118.2(19)$ & $4304.1(10)$ & $32.1(5)$ \\
\hline C69 & 7821.4(17) & $-2735.2(17)$ & $4009.5(10)$ & $27.5(5)$ \\
\hline $\mathrm{C} 70$ & $5750.8(17)$ & $-3327.6(16)$ & $2634.5(10)$ & $25.0(4)$ \\
\hline C71 & 6490.6(19) & $-2590.7(19)$ & $2390.1(11)$ & $35.5(5)$ \\
\hline $\mathrm{C} 72$ & $6458(2)$ & $-2719(2)$ & $1768.1(11)$ & $46.6(7)$ \\
\hline $\mathrm{C} 73$ & $5706(2)$ & $-3569(2)$ & $1389.9(11)$ & $48.9(7)$ \\
\hline C74 & $4978(2)$ & $-4307(2)$ & $1624.7(12)$ & $49.2(7)$ \\
\hline
\end{tabular}




$\begin{array}{lllll}\text { C75 } & 4997(2) & -4189.9(19) & 2246.1(11) & 36.6(5) \\ \text { O1 } & 5276(2) & -2942(3) & -575(2) & 156.1(19) \\ \text { N5 } & 6673(3) & -2355(3) & -925(2) & 98.4(12) \\ \text { C76 } & 6255(4) & -2473(4) & -484(2) & 92.7(13) \\ \text { C77 } & 6996(3) & -2049(4) & 140.8(18) & 95.6(15) \\ \text { C78 } & 7835(4) & -1753(6) & -870(3) & 152(3) \\ \text { C79 } & 5996(4) & -2735(4) & -1590(2) & 113.7(18) \\ \text { O2 } & 10164(3) & -3478(2) & 4030.4(16) & 106.4(11) \\ \text { N6 } & 10386(3) & -4482(3) & 3408.0(19) & 104.5(14) \\ \text { C80 } & 9920(3) & -4326(3) & 3818.4(17) & 67.9(9) \\ \text { C81 } & 9087(4) & -5184(4) & 3993(4) & 149(3) \\ \text { C82 } & 10069(5) & -5468(6) & 3092(5) & 248(6) \\ \text { C83 } & 11275(4) & -3684(5) & 3263(3) & 136(3)\end{array}$

Table 3 Anisotropic Displacement Parameters $\left(\AA^{2} \times 10^{3}\right)$ for shuxz_1105_3. The Anisotropic displacement factor exponent takes the form: $2 \pi^{2}\left[h^{2} a^{* 2} U_{11}+2 h k a * b * U_{12}+\ldots\right]$.

$\begin{array}{lllllll}\text { Atom U11 } & \mathbf{U}_{22} & \mathbf{U}_{33} & \mathbf{U}_{23} & \mathbf{U}_{13} & \mathbf{U}_{12} \\ \mathrm{Ni} 1 & 34.4(2) & 21.98(18) & 20.21(18) & 4.03(14) & 15.98(15) & 15.78(15) \\ \mathrm{Ni} 2 & 38.4(2) & 23.41(18) & 19.93(18) & 5.17(14) & 16.76(15) & 17.89(16) \\ \mathrm{P} 1 & 26.6(3) & 23.2(3) & 18.9(2) & 1.69(19) & 11.7(2) & 13.8(2) \\ \mathrm{P} 2 & 34.1(3) & 23.7(3) & 18.8(2) & 4.2(2) & 10.9(2) & 16.3(2) \\ \mathrm{N} 1 & 40.6(10) & 24.1(9) & 24.5(9) & 7.3(7) & 18.9(8) & 19.6(8) \\ \mathrm{N} 2 & 41.1(10) & 27.8(10) & 21.3(9) & 5.4(7) & 18.3(8) & 19.8(8) \\ \mathrm{C} 1 & 31.3(11) & 26.6(11) & 20.2(10) & 4.7(8) & 10.8(8) & 12.6(9) \\ \mathrm{C} 2 & 27.2(10) & 25.9(11) & 20.4(10) & 4.7(8) & 10.8(8) & 11.2(9) \\ \mathrm{C} 3 & 36.9(12) & 33.0(12) & 24.3(11) & 3.9(9) & 15.4(9) & 17.8(10) \\ \mathrm{C} 4 & 50.4(15) & 46.7(15) & 23.1(11) & 6.8(10) & 20.0(10) & 25.3(12)\end{array}$




\begin{tabular}{|c|c|c|c|c|c|c|}
\hline $\mathrm{C} 5$ & $57.9(16)$ & $46.1(15)$ & $25.2(12)$ & $15.7(10)$ & $20.2(11)$ & $29.3(13)$ \\
\hline C6 & $48.6(14)$ & $33.9(13)$ & $27.7(12)$ & $8.9(10)$ & $14.7(10)$ & $23.5(11)$ \\
\hline $\mathrm{C} 7$ & $28.6(10)$ & $22.1(10)$ & $23.1(10)$ & $2.8(8)$ & $11.3(8)$ & $12.8(9)$ \\
\hline $\mathrm{C} 8$ & $34.6(12)$ & $25.9(11)$ & $29.3(11)$ & $4.5(9)$ & $6.5(9)$ & $18.2(9)$ \\
\hline C9 & $39.4(13)$ & $33.1(13)$ & $37.3(13)$ & $5.2(10)$ & $12.9(11)$ & $16.4(11)$ \\
\hline $\mathrm{C} 10$ & $36.9(14)$ & $38.7(14)$ & $61.8(18)$ & $7.6(13)$ & $17.4(13)$ & $16.4(12)$ \\
\hline C11 & $36.2(14)$ & $45.5(16)$ & $58.7(18)$ & $-3.6(14)$ & $-3.4(13)$ & $14.9(12)$ \\
\hline $\mathrm{C} 12$ & $50.7(17)$ & $59.9(19)$ & $37.0(15)$ & $-3.9(13)$ & $-3.7(13)$ & $18.2(15)$ \\
\hline $\mathrm{C} 13$ & $46.7(15)$ & $45.7(15)$ & $30.9(13)$ & $4.8(11)$ & $7.3(11)$ & $18.4(12)$ \\
\hline $\mathrm{C} 14$ & $42.4(13)$ & $42.5(13)$ & 21.1(11) & $5.5(9)$ & $10.8(9)$ & $26.9(11)$ \\
\hline C15 & $56.7(17)$ & $39.7(15)$ & $78(2)$ & $24.3(14)$ & $24.7(16)$ & $31.3(14)$ \\
\hline $\mathrm{C} 16$ & $72(2)$ & $58(2)$ & 104(3) & $28(2)$ & $26(2)$ & $48.5(19)$ \\
\hline C17 & $83(2)$ & $93(3)$ & $53.5(19)$ & $17.6(18)$ & $17.8(17)$ & $70(2)$ \\
\hline $\mathrm{C} 18$ & $50.3(18)$ & $105(3)$ & $55(2)$ & $-18.3(19)$ & $-13.1(15)$ & $44(2)$ \\
\hline C19 & $49.2(17)$ & $67(2)$ & $58.4(19)$ & $-24.7(16)$ & $-8.8(14)$ & $33.8(16)$ \\
\hline $\mathrm{C} 20$ & $25.4(10)$ & $23.6(10)$ & $22.3(10)$ & $0.8(8)$ & $8.5(8)$ & $12.4(8)$ \\
\hline $\mathrm{C} 21$ & 29.1(11) & 26.1(11) & $25.5(11)$ & $1.3(8)$ & $12.0(9)$ & $14.4(9)$ \\
\hline $\mathrm{C} 22$ & $51.4(14)$ & $39.2(13)$ & $27.5(12)$ & $9.8(10)$ & $17.8(11)$ & $29.7(12)$ \\
\hline $\mathrm{C} 23$ & $60.6(17)$ & $45.2(15)$ & $41.0(14)$ & $14.2(12)$ & $21.6(12)$ & $39.5(14)$ \\
\hline $\mathrm{C} 24$ & $55.7(16)$ & $45.8(15)$ & $44.1(14)$ & $9.7(12)$ & $27.3(12)$ & $38.4(13)$ \\
\hline $\mathrm{C} 25$ & $43.4(13)$ & $38.8(13)$ & $26.6(11)$ & $5.3(10)$ & $19.0(10)$ & $26.2(11)$ \\
\hline $\mathrm{C} 26$ & $33.9(11)$ & $25.9(11)$ & $20.0(10)$ & $4.3(8)$ & $12.9(9)$ & $14.9(9)$ \\
\hline $\mathrm{C} 27$ & $26.4(10)$ & $29.7(11)$ & $22.2(10)$ & $2.8(8)$ & $12.0(8)$ & $13.5(9)$ \\
\hline $\mathrm{C} 28$ & $34.3(13)$ & $32.6(13)$ & $50.8(15)$ & $3.1(11)$ & $2.3(11)$ & $16.6(11)$ \\
\hline C29 & $38.5(15)$ & $34.1(14)$ & $68(2)$ & $0.5(13)$ & $2.3(13)$ & $8.4(12)$ \\
\hline C30 & $28.2(12)$ & $52.6(17)$ & $45.3(15)$ & $7.8(13)$ & $4.2(11)$ & $10.1(12)$ \\
\hline C31 & $33.1(13)$ & $54.2(17)$ & $40.9(14)$ & $14.4(12)$ & $6.8(11)$ & $22.9(12)$ \\
\hline
\end{tabular}




\begin{tabular}{|c|c|c|c|c|c|c|}
\hline C32 & $35.4(12)$ & $35.4(13)$ & $35.4(13)$ & $7.9(10)$ & $11.3(10)$ & $18.7(10)$ \\
\hline C33 & $30.4(11)$ & $27.8(11)$ & $24.2(10)$ & $6.1(8)$ & $14.9(9)$ & 16.3(9) \\
\hline C34 & $34.9(12)$ & $42.3(13)$ & $24.7(11)$ & $3.4(10)$ & $12.5(9)$ & $20.3(11)$ \\
\hline C35 & $54.6(16)$ & $59.2(17)$ & $25.1(12)$ & $10.0(11)$ & $23.2(11)$ & $36.3(14)$ \\
\hline C36 & $50.7(15)$ & $50.3(16)$ & $49.8(16)$ & $26.0(13)$ & $38.0(13)$ & $32.0(13)$ \\
\hline C37 & $37.9(14)$ & $47.6(16)$ & $58.0(18)$ & $11.4(13)$ & $26.4(13)$ & $9.6(12)$ \\
\hline C38 & $38.6(13)$ & $39.2(14)$ & $35.5(13)$ & $0.3(11)$ & $16.4(11)$ & $9.5(11)$ \\
\hline Ni3 & $24.93(18)$ & $18.08(17)$ & $20.04(17)$ & $3.13(13)$ & $9.73(13)$ & $12.85(14)$ \\
\hline $\mathrm{Ni4}$ & $27.85(18)$ & $20.10(18)$ & $21.17(18)$ & $3.15(13)$ & $10.96(14)$ & $15.53(15)$ \\
\hline P3 & $23.9(2)$ & $20.7(2)$ & $20.6(2)$ & $4.15(19)$ & $8.2(2)$ & $12.2(2)$ \\
\hline P4 & $25.7(3)$ & $19.7(2)$ & $24.0(3)$ & $1.7(2)$ & $10.6(2)$ & $14.2(2)$ \\
\hline N3 & $30.1(9)$ & $20.2(8)$ & $22.6(9)$ & $3.4(7)$ & $11.5(7)$ & $16.1(7)$ \\
\hline N4 & $30.8(9)$ & 23.9(9) & $19.6(8)$ & $3.9(7)$ & $10.9(7)$ & $16.4(8)$ \\
\hline C1AA & $65.4(18)$ & $46.5(15)$ & $52.1(16)$ & $24.7(13)$ & $41.2(14)$ & $38.1(14)$ \\
\hline C39 & $25.2(10)$ & $19.6(10)$ & $25.2(11)$ & $2.2(8)$ & $8.9(8)$ & $12.0(8)$ \\
\hline $\mathrm{C} 40$ & $33.1(11)$ & $29.8(11)$ & $29.6(11)$ & $5.3(9)$ & $17.6(9)$ & $18.5(10)$ \\
\hline $\mathrm{C} 41$ & $32.7(11)$ & $29.2(11)$ & $27.8(11)$ & $4.1(9)$ & 16.8(9) & 18.2(9) \\
\hline C42 & $60.9(17)$ & $41.6(14)$ & $48.5(15)$ & $13.6(12)$ & $35.0(13)$ & $36.6(13)$ \\
\hline $\mathrm{C} 43$ & $103(3)$ & $73(2)$ & $67(2)$ & $30.2(17)$ & $67(2)$ & $68(2)$ \\
\hline $\mathrm{C} 44$ & 110(3) & $73(2)$ & $71(2)$ & 43.1(18) & $78(2)$ & $65(2)$ \\
\hline $\mathrm{C} 45$ & $28.9(11)$ & $30.1(11)$ & $24.1(10)$ & $-0.2(9)$ & $8.8(9)$ & 18.9(9) \\
\hline $\mathrm{C} 46$ & $31.6(12)$ & $38.9(14)$ & $47.7(15)$ & $1.5(11)$ & $8.2(11)$ & $17.0(11)$ \\
\hline C47 & $37.1(14)$ & $71(2)$ & 47.1(16) & $-0.2(14)$ & $1.7(12)$ & $34.9(14)$ \\
\hline $\mathrm{C} 48$ & $61.2(18)$ & $70(2)$ & $41.8(15)$ & $8.9(14)$ & $8.9(13)$ & $55.1(17)$ \\
\hline C49 & $62.1(18)$ & $40.8(15)$ & $58.0(18)$ & 10.1(13) & $11.3(14)$ & $37.6(14)$ \\
\hline $\mathrm{C} 50$ & $39.0(13)$ & $29.3(12)$ & $43.3(14)$ & $1.3(10)$ & 7.1(11) & $20.9(11)$ \\
\hline C51 & $30.0(11)$ & $23.3(11)$ & $31.3(11)$ & $2.2(9)$ & $4.0(9)$ & $17.8(9)$ \\
\hline
\end{tabular}




\begin{tabular}{|c|c|c|c|c|c|c|}
\hline C52 & $33.2(12)$ & $36.0(13)$ & $44.2(14)$ & $3.8(11)$ & $10.4(11)$ & $17.6(11)$ \\
\hline C53 & $30.3(13)$ & $40.3(15)$ & $75(2)$ & $8.7(14)$ & $11.0(13)$ & $15.0(11)$ \\
\hline C54 & $39.3(15)$ & $31.5(14)$ & $67(2)$ & $-2.7(13)$ & $-11.5(14)$ & $15.0(12)$ \\
\hline C55 & $60.5(18)$ & $40.4(15)$ & 41.1(15) & $-8.3(12)$ & $-10.2(13)$ & $25.8(14)$ \\
\hline $\mathrm{C} 56$ & $46.8(14)$ & $35.3(13)$ & $33.3(13)$ & $-1.3(10)$ & $4.7(11)$ & $22.4(11)$ \\
\hline C57 & $26.9(10)$ & $22.2(10)$ & $22.0(10)$ & $2.7(8)$ & $5.4(8)$ & $13.9(9)$ \\
\hline C58 & $23.3(10)$ & $19.2(10)$ & $24.2(10)$ & $2.3(8)$ & $5.8(8)$ & $10.6(8)$ \\
\hline C59 & $36.9(12)$ & $30.1(12)$ & $25.0(11)$ & $7.9(9)$ & $10.5(9)$ & $18.9(10)$ \\
\hline $\mathrm{C} 60$ & $43.6(13)$ & $31.6(12)$ & $28.7(12)$ & 7.8(9) & $4.0(10)$ & $23.5(11)$ \\
\hline C61 & $40.4(13)$ & $33.4(12)$ & $36.0(13)$ & $2.9(10)$ & $5.9(10)$ & $27.5(11)$ \\
\hline C62 & $35.5(12)$ & $30.5(12)$ & $30.2(12)$ & $3.6(9)$ & $10.8(9)$ & $21.7(10)$ \\
\hline C63 & $24.1(10)$ & $23.2(10)$ & $23.2(10)$ & $2.4(8)$ & $9.1(8)$ & $13.3(8)$ \\
\hline C64 & $25.7(10)$ & $25.9(11)$ & 17.3(9) & $3.6(8)$ & $7.5(8)$ & 11.9(9) \\
\hline C65 & $30.4(11)$ & $30.9(12)$ & $28.7(11)$ & $0.8(9)$ & 7.6(9) & $15.7(10)$ \\
\hline C66 & $35.4(13)$ & $31.2(12)$ & $33.8(13)$ & $-7.9(10)$ & $8.5(10)$ & $10.1(10)$ \\
\hline C67 & $28.1(11)$ & $46.6(14)$ & $20.6(11)$ & $-5.9(10)$ & $4.4(9)$ & $11.3(10)$ \\
\hline C68 & $29.7(11)$ & 49.7(15) & $21.4(11)$ & $6.1(10)$ & $7.5(9)$ & $21.2(11)$ \\
\hline C69 & $32.7(11)$ & $31.6(12)$ & $23.7(11)$ & $6.9(9)$ & 11.1(9) & $17.4(10)$ \\
\hline C70 & $28.6(11)$ & $28.6(11)$ & $23.3(10)$ & $2.8(8)$ & $7.0(8)$ & $17.6(9)$ \\
\hline C71 & $33.4(12)$ & $43.0(14)$ & $26.0(12)$ & $4.0(10)$ & $8.5(10)$ & $12.9(11)$ \\
\hline C72 & $43.5(15)$ & $70.2(19)$ & $25.7(13)$ & $10.1(12)$ & $14.8(11)$ & $22.1(14)$ \\
\hline C73 & $55.3(17)$ & $82(2)$ & $19.9(12)$ & $3.3(13)$ & $9.1(11)$ & $40.8(16)$ \\
\hline C74 & $52.5(16)$ & $58.4(18)$ & $27.5(13)$ & $-11.8(12)$ & $-6.9(12)$ & $25.8(14)$ \\
\hline C75 & $37.2(13)$ & 35.7(13) & $31.2(12)$ & $-0.3(10)$ & $4.6(10)$ & $13.4(11)$ \\
\hline $\mathrm{O} 1$ & $54.2(18)$ & 172(4) & $175(4)$ & $65(3)$ & $41(2)$ & $-20(2)$ \\
\hline N5 & 101(3) & $114(3)$ & $125(3)$ & $52(3)$ & $67(3)$ & $68(3)$ \\
\hline C76 & $96(3)$ & $86(3)$ & 107(4) & $36(3)$ & $40(3)$ & $42(3)$ \\
\hline
\end{tabular}




$\begin{array}{lllllll}\text { C77 } & 83(3) & 142(4) & 60(2) & 41(3) & 17(2) & 46(3) \\ \text { C78 } & 65(3) & 283(9) & 138(5) & 96(6) & 60(3) & 84(4) \\ \text { C79 } & 121(4) & 123(4) & 69(3) & -17(3) & 9(3) & 38(3) \\ \text { O2 } & 127(3) & 85(2) & 107(2) & -27.6(18) & 1(2) & 64(2) \\ \text { N6 } & 67(2) & 127(3) & 106(3) & -44(3) & 6(2) & 44(2) \\ \text { C80 } & 64(2) & 72(2) & 65(2) & -9.6(18) & 1.9(17) & 37.0(19) \\ \text { C81 } & 97(4) & 111(4) & 270(9) & 75(5) & 100(5) & 48(3) \\ \text { C82 } & 118(5) & 222(8) & 328(12) & -203(9) & 44(6) & 39(5) \\ \text { C83 } & 69(3) & 211(7) & 152(5) & 96(5) & 45(3) & 69(4)\end{array}$

Table 4 Bond Lengths for shuxz_1105_3.

Atom Atom Length/Å

Ni1 P1 2.1338(6)

Ni1 N1 1.8862(17)

Ni1 N2 1.8964(18)

Ni1 C7 1.830(2)

$\mathrm{Ni2} \quad \mathrm{P} 2 \quad 2.1333(6)$

Ni2 N1 1.8941(17)

$\mathrm{Ni} 2 \quad \mathrm{~N} 2^{1} \quad 1.8932(17)$

$\mathrm{Ni} 2 \mathrm{C}^{2} 6^{1} 1.831(2)$

P1 C20 1.837(2)

$\mathrm{P} 1 \quad \mathrm{C} 27 \quad 1.833(2)$

P1 C33 1.825(2)

P2 C1 1.837(2)

$\mathrm{P} 2 \quad \mathrm{C} 8 \quad 1.834(2)$

P2 C14 1.829(2)

$\mathrm{N} 1 \quad \mathrm{C} 7 \quad 1.239(3)$

\section{Atom Atom Length/Å}

Ni4 N3 1.9037(17)

$\mathrm{Ni4} \quad \mathrm{N} 4^{2} \quad 1.8964(17)$

$\mathrm{Ni} 4 \quad \mathrm{C}^{2} 3^{2} 1.836(2)$

P3 $\quad$ C58 1.836(2)

P3 C64 1.837(2)

P3 C70 1.826(2)

P4 C41 1.838(2)

P4 C45 1.824(2)

P4 C51 1.837(2)

N3 $\quad$ C39 $1.237(3)$

N4 C63 1.239(3)

C1AAC40 1.396(3)

C1AAC44 1.382(4)

C39 C40 1.468(3)

C40 C41 1.407(3) 


\begin{tabular}{|c|c|c|c|c|c|}
\hline $\mathrm{N} 2$ & $\mathrm{C} 26$ & $1.238(3)$ & C41 & $\mathrm{C} 42$ & $1.398(3)$ \\
\hline $\mathrm{C} 1$ & $\mathrm{C} 2$ & $1.405(3)$ & $\mathrm{C} 42$ & $\mathrm{C} 43$ & $1.381(4)$ \\
\hline $\mathrm{C} 1$ & C6 & $1.397(3)$ & $\mathrm{C} 43$ & $\mathrm{C} 44$ & $1.381(4)$ \\
\hline $\mathrm{C} 2$ & $\mathrm{C} 3$ & $1.400(3)$ & $\mathrm{C} 45$ & $\mathrm{C} 46$ & $1.392(3)$ \\
\hline $\mathrm{C} 2$ & $\mathrm{C} 7$ & $1.465(3)$ & $\mathrm{C} 45$ & C50 & $1.383(3)$ \\
\hline $\mathrm{C} 3$ & $\mathrm{C} 4$ & $1.382(3)$ & $\mathrm{C} 46$ & $\mathrm{C} 47$ & $1.385(4)$ \\
\hline $\mathrm{C} 4$ & C5 & $1.379(4)$ & C47 & $\mathrm{C} 48$ & $1.380(4)$ \\
\hline $\mathrm{C} 5$ & C6 & $1.392(3)$ & $\mathrm{C} 48$ & C49 & $1.365(4)$ \\
\hline $\mathrm{C} 8$ & C9 & $1.386(3)$ & C49 & C50 & $1.386(4)$ \\
\hline $\mathrm{C} 8$ & $\mathrm{C} 13$ & $1.396(3)$ & C51 & C52 & $1.390(3)$ \\
\hline C9 & $\mathrm{C} 10$ & $1.391(4)$ & C51 & C56 & $1.398(3)$ \\
\hline $\mathrm{C} 10$ & $\mathrm{C} 11$ & $1.371(4)$ & C52 & C53 & $1.394(4)$ \\
\hline C11 & $\mathrm{C} 12$ & $1.386(4)$ & C53 & C54 & $1.373(4)$ \\
\hline $\mathrm{C} 12$ & C13 & $1.383(4)$ & C54 & C55 & $1.372(5)$ \\
\hline $\mathrm{C} 14$ & $\mathrm{C} 15$ & $1.381(4)$ & C55 & C56 & $1.387(4)$ \\
\hline $\mathrm{C} 14$ & $\mathrm{C} 19$ & $1.365(4)$ & C57 & C58 & $1.413(3)$ \\
\hline $\mathrm{C} 15$ & C16 & $1.383(4)$ & C57 & C62 & $1.392(3)$ \\
\hline $\mathrm{C} 16$ & $\mathrm{C} 17$ & $1.340(5)$ & C57 & C63 & $1.470(3)$ \\
\hline $\mathrm{C} 17$ & $\mathrm{C} 18$ & $1.363(5)$ & C58 & C59 & $1.399(3)$ \\
\hline $\mathrm{C} 18$ & C19 & $1.400(5)$ & C59 & C60 & $1.386(3)$ \\
\hline $\mathrm{C} 20$ & $\mathrm{C} 21$ & $1.409(3)$ & C60 & C61 & $1.379(3)$ \\
\hline $\mathrm{C} 20$ & $\mathrm{C} 25$ & $1.393(3)$ & C61 & C62 & $1.386(3)$ \\
\hline $\mathrm{C} 21$ & $\mathrm{C} 22$ & $1.393(3)$ & C64 & C65 & $1.389(3)$ \\
\hline $\mathrm{C} 21$ & $\mathrm{C} 26$ & $1.465(3)$ & C64 & C69 & $1.395(3)$ \\
\hline $\mathrm{C} 22$ & $\mathrm{C} 23$ & $1.380(3)$ & C65 & C66 & $1.389(3)$ \\
\hline $\mathrm{C} 23$ & $\mathrm{C} 24$ & $1.383(4)$ & C66 & C67 & $1.380(4)$ \\
\hline $\mathrm{C} 24$ & $\mathrm{C} 25$ & $1.381(3)$ & C67 & C68 & $1.379(4)$ \\
\hline
\end{tabular}




\begin{tabular}{|c|c|c|c|c|c|}
\hline $\mathrm{C} 27$ & $\mathrm{C} 28$ & $1.383(3)$ & C68 & C69 & $1.390(3)$ \\
\hline $\mathrm{C} 27$ & C32 & $1.395(3)$ & C70 & C71 & $1.397(3)$ \\
\hline $\mathrm{C} 28$ & $\mathrm{C} 29$ & $1.390(4)$ & C70 & C75 & $1.388(3)$ \\
\hline $\mathrm{C} 29$ & $\mathrm{C} 30$ & $1.375(4)$ & C71 & C72 & $1.387(3)$ \\
\hline $\mathrm{C} 30$ & C31 & $1.374(4)$ & $\mathrm{C} 72$ & C73 & $1.367(4)$ \\
\hline C31 & $\mathrm{C} 32$ & $1.382(3)$ & C73 & C74 & $1.377(4)$ \\
\hline $\mathrm{C} 33$ & C34 & $1.385(3)$ & C74 & C75 & $1.389(4)$ \\
\hline C33 & C38 & $1.391(3)$ & $\mathrm{O} 1$ & C76 & $1.246(5)$ \\
\hline C34 & C35 & $1.403(3)$ & N5 & C76 & $1.240(6)$ \\
\hline $\mathrm{C} 35$ & C36 & $1.366(4)$ & N5 & C78 & $1.501(6)$ \\
\hline C36 & C37 & $1.381(4)$ & N5 & C79 & $1.537(6)$ \\
\hline C37 & C38 & $1.384(4)$ & C76 & C77 & $1.498(6)$ \\
\hline $\mathrm{Ni3}$ & P3 & $2.1430(6)$ & $\mathrm{O} 2$ & C80 & $1.212(4)$ \\
\hline $\mathrm{Ni3}$ & N3 & $1.8896(17)$ & N6 & $\mathrm{C} 80$ & $1.308(5)$ \\
\hline $\mathrm{Ni3}$ & N4 & $1.9149(17)$ & N6 & $\mathrm{C} 82$ & $1.450(7)$ \\
\hline Ni3 & C39 & $1.835(2)$ & N6 & $\mathrm{C} 83$ & $1.442(6)$ \\
\hline $\mathrm{Ni} 4$ & P4 & $2.1386(6)$ & $\mathrm{C} 80$ & C81 & $1.475(6)$ \\
\hline
\end{tabular}

Table 5 Bond Angles for shuxz_1105_3.

\section{Atom Atom Atom Angle ${ }^{\circ}$}

N1 Ni1 P1 154.55(6)

N1 Ni1 N2 111.41(7)

N2 Ni1 P1 92.81(6)

C7 Ni1 P1 116.71(7)

C7 Ni1 N1 38.92(8)

C7 Ni1 N2 150.31(8)
Atom Atom Atom Angle $/^{\circ}$

N3 Ni4 P4 91.91(5)

$\mathrm{N}^{2} \quad \mathrm{Ni} 4 \quad \mathrm{P} 4 \quad 155.79(6)$

$\mathrm{N}^{2} \quad \mathrm{Ni} 4 \quad \mathrm{~N} 3 \quad 112.25(7)$

${\mathrm{C} 63^{2}}^{2} \mathrm{Ni} 4 \quad \mathrm{P} 4 \quad 117.29(7)$

${\mathrm{C} 63^{2}}^{2} \mathrm{Ni} 4 \mathrm{~N} 3 \quad 150.65(8)$

${\mathrm{C} 63^{2}}^{2} \mathrm{Ni} 4 \quad \mathrm{~N} 4^{2} \quad 38.73(8)$ 


\begin{tabular}{|c|c|c|c|c|c|c|c|}
\hline N1 & $\mathrm{Ni} 2$ & $\mathrm{P} 2$ & $92.68(6)$ & C58 & P3 & Ni3 & $114.56(7)$ \\
\hline $\mathrm{N} 2^{1}$ & $\mathrm{Ni} 2$ & $\mathrm{P} 2$ & $153.94(6)$ & C58 & P3 & C64 & $103.87(9)$ \\
\hline $\mathrm{N} 2^{1}$ & $\mathrm{Ni} 2$ & N1 & $112.60(8)$ & C64 & P3 & Ni3 & $107.53(7)$ \\
\hline $\mathrm{C} 26^{1}$ & $\mathrm{Ni} 2$ & $\mathrm{P} 2$ & $115.86(7)$ & $\mathrm{C} 70$ & $\mathrm{P} 3$ & $\mathrm{Ni3}$ & $122.41(7)$ \\
\hline $\mathrm{C} 26^{1}$ & $\mathrm{Ni} 2$ & N1 & $151.36(9)$ & C70 & P3 & $\mathrm{C} 58$ & $103.16(10)$ \\
\hline $\mathrm{C} 26^{1}$ & $\mathrm{Ni} 2$ & $\mathrm{~N} 2^{1}$ & $38.78(9)$ & $\mathrm{C} 70$ & $\mathrm{P} 3$ & C64 & $103.37(9)$ \\
\hline $\mathrm{C} 20$ & $\mathrm{P} 1$ & Ni1 & $114.29(7)$ & $\mathrm{C} 41$ & $\mathrm{P} 4$ & $\mathrm{Ni} 4$ & $114.15(7)$ \\
\hline $\mathrm{C} 27$ & $\mathrm{P} 1$ & Nil & $109.53(7)$ & $\mathrm{C} 45$ & $\mathrm{P} 4$ & $\mathrm{Ni4}$ & $118.15(7)$ \\
\hline $\mathrm{C} 27$ & $\mathrm{P} 1$ & $\mathrm{C} 20$ & $103.46(10)$ & $\mathrm{C} 45$ & $\mathrm{P} 4$ & $\mathrm{C} 41$ & $103.52(10)$ \\
\hline C33 & $\mathrm{P} 1$ & Ni1 & $121.75(7)$ & $\mathrm{C} 45$ & $\mathrm{P} 4$ & C51 & $103.47(10)$ \\
\hline C33 & $\mathrm{P} 1$ & $\mathrm{C} 20$ & $101.89(10)$ & C51 & $\mathrm{P} 4$ & $\mathrm{Ni4}$ & $112.93(7)$ \\
\hline C33 & $\mathrm{P} 1$ & $\mathrm{C} 27$ & $104.01(10)$ & C51 & $\mathrm{P} 4$ & C41 & $102.89(10)$ \\
\hline $\mathrm{C} 1$ & $\mathrm{P} 2$ & $\mathrm{Ni} 2$ & $115.53(7)$ & $\mathrm{Ni3}$ & $\mathrm{N} 3$ & $\mathrm{Ni} 4$ & $156.09(10)$ \\
\hline $\mathrm{C} 8$ & $\mathrm{P} 2$ & $\mathrm{Ni} 2$ & $108.89(7)$ & C39 & N3 & $\mathrm{Ni3}$ & $68.25(12)$ \\
\hline $\mathrm{C} 8$ & $\mathrm{P} 2$ & $\mathrm{C} 1$ & $103.36(10)$ & C39 & N3 & $\mathrm{Ni} 4$ & $133.70(15)$ \\
\hline $\mathrm{C} 14$ & $\mathrm{P} 2$ & $\mathrm{Ni} 2$ & $120.02(8)$ & $\mathrm{Ni}^{2}$ & $\mathrm{~N} 4$ & $\mathrm{Ni3}$ & $156.56(10)$ \\
\hline C14 & $\mathrm{P} 2$ & $\mathrm{C} 1$ & $103.48(10)$ & C63 & N4 & $\mathrm{Ni3}$ & $133.82(15)$ \\
\hline $\mathrm{C} 14$ & $\mathrm{P} 2$ & $\mathrm{C} 8$ & $103.75(11)$ & C63 & N4 & $\mathrm{Ni}^{2}$ & $67.98(12)$ \\
\hline Ni1 & N1 & $\mathrm{Ni} 2$ & $157.52(10)$ & $\mathrm{C} 44$ & \multicolumn{2}{|c|}{ C1AAC40 } & $120.4(2)$ \\
\hline $\mathrm{C} 7$ & N1 & Ni1 & $68.10(12)$ & N3 & C39 & $\mathrm{Ni3}$ & $73.00(12)$ \\
\hline $\mathrm{C} 7$ & N1 & $\mathrm{Ni} 2$ & $133.94(16)$ & N3 & C39 & $\mathrm{C} 40$ & $132.3(2)$ \\
\hline $\mathrm{Ni} 2^{1}$ & $\mathrm{~N} 2$ & Ni1 & $157.69(10)$ & $\mathrm{C} 40$ & C39 & $\mathrm{Ni3}$ & $154.56(17)$ \\
\hline $\mathrm{C} 26$ & $\mathrm{~N} 2$ & Ni1 & $134.03(15)$ & \multicolumn{2}{|c|}{ C1AAC40 } & C39 & $118.7(2)$ \\
\hline $\mathrm{C} 26$ & $\mathrm{~N} 2$ & $\mathrm{Ni} 2^{1}$ & $67.90(12)$ & \multicolumn{2}{|c|}{ C1AAC40 } & $\mathrm{C} 41$ & $119.9(2)$ \\
\hline $\mathrm{C} 2$ & $\mathrm{C} 1$ & $\mathrm{P} 2$ & $120.27(16)$ & $\mathrm{C} 41$ & $\mathrm{C} 40$ & C39 & $121.41(19)$ \\
\hline C6 & $\mathrm{C} 1$ & $\mathrm{P} 2$ & $121.02(17)$ & $\mathrm{C} 40$ & $\mathrm{C} 41$ & $\mathrm{P} 4$ & $120.91(16)$ \\
\hline C6 & $\mathrm{C} 1$ & $\mathrm{C} 2$ & $118.7(2)$ & $\mathrm{C} 42$ & $\mathrm{C} 41$ & $\mathrm{P} 4$ & $120.69(18)$ \\
\hline
\end{tabular}




\begin{tabular}{|c|c|c|c|c|c|c|c|}
\hline $\mathrm{C} 1$ & $\mathrm{C} 2$ & $\mathrm{C} 7$ & $121.87(18)$ & $\mathrm{C} 42$ & C41 & $\mathrm{C} 40$ & $118.4(2)$ \\
\hline $\mathrm{C} 3$ & $\mathrm{C} 2$ & $\mathrm{C} 1$ & $119.6(2)$ & $\mathrm{C} 43$ & $\mathrm{C} 42$ & C41 & $121.0(2)$ \\
\hline $\mathrm{C} 3$ & $\mathrm{C} 2$ & $\mathrm{C} 7$ & $118.50(19)$ & $\mathrm{C} 42$ & $\mathrm{C} 43$ & $\mathrm{C} 44$ & $120.2(2)$ \\
\hline $\mathrm{C} 4$ & $\mathrm{C} 3$ & $\mathrm{C} 2$ & $120.5(2)$ & $\mathrm{C} 43$ & $\mathrm{C} 44$ & $\mathrm{C} 1 \mathrm{AA}$ & $120.1(2)$ \\
\hline $\mathrm{C} 5$ & $\mathrm{C} 4$ & $\mathrm{C} 3$ & $120.4(2)$ & $\mathrm{C} 46$ & $\mathrm{C} 45$ & $\mathrm{P} 4$ & $118.65(18)$ \\
\hline $\mathrm{C} 4$ & $\mathrm{C} 5$ & C6 & $119.6(2)$ & C50 & $\mathrm{C} 45$ & $\mathrm{P} 4$ & $122.26(17)$ \\
\hline $\mathrm{C} 5$ & C6 & $\mathrm{C} 1$ & $121.1(2)$ & C50 & $\mathrm{C} 45$ & $\mathrm{C} 46$ & $118.7(2)$ \\
\hline $\mathrm{N} 1$ & $\mathrm{C} 7$ & Ni1 & $72.99(13)$ & $\mathrm{C} 47$ & $\mathrm{C} 46$ & $\mathrm{C} 45$ & $120.4(3)$ \\
\hline N1 & $\mathrm{C} 7$ & $\mathrm{C} 2$ & $133.0(2)$ & $\mathrm{C} 48$ & $\mathrm{C} 47$ & $\mathrm{C} 46$ & $120.1(3)$ \\
\hline $\mathrm{C} 2$ & $\mathrm{C} 7$ & Ni1 & 154.01(16) & C49 & $\mathrm{C} 48$ & $\mathrm{C} 47$ & $119.6(2)$ \\
\hline C9 & $\mathrm{C} 8$ & $\mathrm{P} 2$ & $124.57(18)$ & $\mathrm{C} 48$ & C49 & $\mathrm{C} 50$ & $120.8(3)$ \\
\hline C9 & $\mathrm{C} 8$ & $\mathrm{C} 13$ & $118.6(2)$ & $\mathrm{C} 45$ & C50 & C49 & $120.3(2)$ \\
\hline $\mathrm{C} 13$ & $\mathrm{C} 8$ & $\mathrm{P} 2$ & $116.78(19)$ & C52 & C51 & $\mathrm{P} 4$ & $118.24(18)$ \\
\hline $\mathrm{C} 8$ & C9 & $\mathrm{C} 10$ & $120.7(2)$ & C52 & C51 & C56 & $118.3(2)$ \\
\hline $\mathrm{C} 11$ & $\mathrm{C} 10$ & C9 & $120.2(3)$ & C56 & C51 & $\mathrm{P} 4$ & $123.45(19)$ \\
\hline $\mathrm{C} 10$ & C11 & $\mathrm{C} 12$ & 119.7(3) & C51 & C52 & C53 & $120.5(3)$ \\
\hline $\mathrm{C} 13$ & $\mathrm{C} 12$ & $\mathrm{C} 11$ & $120.4(3)$ & C54 & C53 & C52 & $120.3(3)$ \\
\hline $\mathrm{C} 12$ & $\mathrm{C} 13$ & $\mathrm{C} 8$ & $120.3(3)$ & C55 & C54 & C53 & $120.0(3)$ \\
\hline C15 & C14 & $\mathrm{P} 2$ & $122.3(2)$ & C54 & C55 & C56 & $120.4(3)$ \\
\hline C19 & $\mathrm{C} 14$ & $\mathrm{P} 2$ & $119.0(2)$ & C55 & C56 & C51 & $120.5(3)$ \\
\hline C19 & $\mathrm{C} 14$ & $\mathrm{C} 15$ & $118.3(3)$ & C58 & C57 & C63 & $120.48(18)$ \\
\hline C14 & C15 & C16 & $120.8(3)$ & C62 & C57 & C58 & $119.93(19)$ \\
\hline C17 & C16 & C15 & $120.3(3)$ & C62 & C57 & C63 & $119.52(19)$ \\
\hline $\mathrm{C} 16$ & $\mathrm{C} 17$ & $\mathrm{C} 18$ & $120.3(3)$ & C57 & C58 & P3 & $119.48(15)$ \\
\hline $\mathrm{C} 17$ & $\mathrm{C} 18$ & C19 & $119.9(3)$ & C59 & C58 & P3 & $122.33(16)$ \\
\hline C14 & C19 & $\mathrm{C} 18$ & $120.3(3)$ & C59 & C58 & C57 & $118.19(19)$ \\
\hline C21 & $\mathrm{C} 20$ & $\mathrm{P} 1$ & $120.83(16)$ & C60 & C59 & C58 & $121.2(2)$ \\
\hline
\end{tabular}




\begin{tabular}{|c|c|c|c|c|c|c|c|}
\hline $\mathrm{C} 25$ & $\mathrm{C} 20$ & $\mathrm{P} 1$ & $121.09(16)$ & C61 & C60 & C59 & $120.1(2)$ \\
\hline $\mathrm{C} 25$ & $\mathrm{C} 20$ & $\mathrm{C} 21$ & $118.1(2)$ & C60 & C61 & C62 & $120.0(2)$ \\
\hline $\mathrm{C} 20$ & $\mathrm{C} 21$ & $\mathrm{C} 26$ & $121.2(2)$ & C61 & C62 & $\mathrm{C} 57$ & $120.6(2)$ \\
\hline $\mathrm{C} 22$ & $\mathrm{C} 21$ & $\mathrm{C} 20$ & $119.8(2)$ & N4 & C63 & $\mathrm{Ni} 4^{2}$ & $73.29(13)$ \\
\hline $\mathrm{C} 22$ & $\mathrm{C} 21$ & $\mathrm{C} 26$ & $118.8(2)$ & N4 & C63 & $\mathrm{C} 57$ & 132.01(19) \\
\hline $\mathrm{C} 23$ & $\mathrm{C} 22$ & $\mathrm{C} 21$ & $120.9(2)$ & C57 & C63 & $\mathrm{Ni}_{4}^{2}$ & $154.69(16)$ \\
\hline $\mathrm{C} 22$ & $\mathrm{C} 23$ & $\mathrm{C} 24$ & $119.6(2)$ & C65 & C64 & P3 & $123.74(16)$ \\
\hline $\mathrm{C} 25$ & $\mathrm{C} 24$ & $\mathrm{C} 23$ & $120.1(2)$ & C65 & C64 & C69 & $119.0(2)$ \\
\hline $\mathrm{C} 24$ & $\mathrm{C} 25$ & $\mathrm{C} 20$ & $121.5(2)$ & C69 & C64 & P3 & $117.26(16)$ \\
\hline $\mathrm{N} 2$ & $\mathrm{C} 26$ & $\mathrm{Ni} 2^{1}$ & $73.32(13)$ & C64 & C65 & C66 & $120.3(2)$ \\
\hline $\mathrm{N} 2$ & $\mathrm{C} 26$ & $\mathrm{C} 21$ & $132.3(2)$ & C67 & C66 & C65 & $119.9(2)$ \\
\hline $\mathrm{C} 21$ & $\mathrm{C} 26$ & $\mathrm{Ni} 2^{1}$ & $154.29(17)$ & C68 & C67 & C66 & $120.7(2)$ \\
\hline $\mathrm{C} 28$ & $\mathrm{C} 27$ & $\mathrm{P} 1$ & $123.86(18)$ & C67 & C68 & C69 & $119.4(2)$ \\
\hline $\mathrm{C} 28$ & $\mathrm{C} 27$ & C32 & $118.3(2)$ & C68 & C69 & C64 & $120.7(2)$ \\
\hline $\mathrm{C} 32$ & $\mathrm{C} 27$ & $\mathrm{P} 1$ & $117.83(17)$ & C71 & C70 & P3 & $122.99(17)$ \\
\hline $\mathrm{C} 27$ & $\mathrm{C} 28$ & $\mathrm{C} 29$ & $120.6(2)$ & $\mathrm{C} 75$ & C70 & $\mathrm{P} 3$ & $118.15(18)$ \\
\hline $\mathrm{C} 30$ & C29 & $\mathrm{C} 28$ & $120.5(3)$ & $\mathrm{C} 75$ & C70 & C71 & $118.9(2)$ \\
\hline C31 & $\mathrm{C} 30$ & $\mathrm{C} 29$ & $119.4(2)$ & $\mathrm{C} 72$ & C71 & $\mathrm{C} 70$ & $120.2(2)$ \\
\hline $\mathrm{C} 30$ & C31 & C32 & $120.6(2)$ & C73 & C72 & C71 & $120.3(3)$ \\
\hline C31 & C32 & $\mathrm{C} 27$ & $120.6(2)$ & C72 & C73 & C74 & $120.2(2)$ \\
\hline C34 & $\mathrm{C} 33$ & $\mathrm{P} 1$ & $123.75(17)$ & C73 & C74 & $\mathrm{C} 75$ & $120.2(3)$ \\
\hline C34 & C33 & C38 & $118.6(2)$ & C70 & C75 & C74 & $120.1(2)$ \\
\hline C38 & $\mathrm{C} 33$ & $\mathrm{P} 1$ & $117.59(17)$ & C76 & N5 & C78 & $124.3(5)$ \\
\hline C33 & C34 & C35 & $120.2(2)$ & C76 & N5 & C79 & $120.8(4)$ \\
\hline C36 & $\mathrm{C} 35$ & C34 & $120.3(2)$ & C78 & N5 & C79 & $114.6(4)$ \\
\hline C35 & C36 & C37 & $119.9(2)$ & $\mathrm{O} 1$ & C76 & C77 & $124.1(5)$ \\
\hline C36 & C37 & $\mathrm{C} 38$ & $120.1(3)$ & N5 & C76 & $\mathrm{O} 1$ & $120.0(5)$ \\
\hline
\end{tabular}




\begin{tabular}{|c|c|c|c|c|c|c|c|}
\hline $\mathrm{C} 37$ & C38 & C33 & $120.8(2)$ & N5 & C76 & C77 & $115.9(5)$ \\
\hline N3 & $\mathrm{Ni3}$ & P3 & $155.64(5)$ & $\mathrm{C} 80$ & N6 & $\mathrm{C} 82$ & $122.0(5)$ \\
\hline N3 & $\mathrm{Ni3}$ & N4 & $112.11(7)$ & $\mathrm{C} 80$ & N6 & $\mathrm{C} 83$ & $121.7(4)$ \\
\hline N4 & $\mathrm{Ni3}$ & P3 & $90.58(5)$ & $\mathrm{C} 83$ & N6 & $\mathrm{C} 82$ & $116.2(5)$ \\
\hline C39 & $\mathrm{Ni3}$ & $\mathrm{P} 3$ & $117.87(7)$ & $\mathrm{O} 2$ & $\mathrm{C} 80$ & N6 & $118.9(4)$ \\
\hline C39 & $\mathrm{Ni3}$ & N3 & $38.75(8)$ & $\mathrm{O} 2$ & $\mathrm{C} 80$ & C81 & $121.8(5)$ \\
\hline C39 & $\mathrm{Ni3}$ & N4 & $150.78(8)$ & N6 & $\mathrm{C} 80$ & C81 & $119.2(4)$ \\
\hline
\end{tabular}

Table 6 Torsion Angles for shuxz_1105_3.

\begin{tabular}{|c|c|c|c|c|c|c|c|}
\hline $\mathbf{A}$ & B & C D & Angle $/^{\circ}$ & $\mathbf{A}$ & B & C D & Angle $/^{\circ}$ \\
\hline Ni1 & $\mathrm{P} 1$ & $\mathrm{C} 20 \mathrm{C} 21$ & $-20.31(19)$ & C36 & C37 & C38C33 & $-1.6(4)$ \\
\hline Ni1 & $\mathrm{P} 1$ & $\mathrm{C} 20 \mathrm{C} 25$ & $158.38(16)$ & C38 & C33 & C34C35 & $0.7(4)$ \\
\hline Ni1 & $\mathrm{P} 1$ & $\mathrm{C} 27 \mathrm{C} 28$ & $132.27(19)$ & $\mathrm{Ni3}$ & $\mathrm{P} 3$ & C58C57 & 26.61(18) \\
\hline Ni1 & $\mathrm{P} 1$ & $\mathrm{C} 27 \mathrm{C} 32$ & $-46.66(18)$ & Ni3 & $\mathrm{P} 3$ & C58C59 & $-152.30(16)$ \\
\hline Ni1 & $\mathrm{P} 1$ & C33C34 & $134.15(18)$ & Ni3 & $\mathrm{P} 3$ & C64C65 & $-137.73(17)$ \\
\hline Ni1 & $\mathrm{P} 1$ & C33 C38 & $-47.7(2)$ & $\mathrm{Ni3}$ & $\mathrm{P} 3$ & C64 C69 & 41.94(17) \\
\hline Ni1 & N1 & $\mathrm{C} 7 \mathrm{C} 2$ & $-178.2(3)$ & Ni3 & $\mathrm{P} 3$ & C70C71 & $-145.29(17)$ \\
\hline Ni1 & $\mathrm{N} 2$ & $\mathrm{C} 26 \mathrm{Ni} 2^{1}$ & ${ }^{1} 175.0(2)$ & $\mathrm{Ni3}$ & $\mathrm{P} 3$ & $\mathrm{C} 70 \mathrm{C} 75$ & $33.8(2)$ \\
\hline Ni1 & $\mathrm{N} 2$ & $\mathrm{C} 26 \mathrm{C} 21$ & $-7.2(4)$ & $\mathrm{Ni3}$ & N3 & C39C40 & $176.6(3)$ \\
\hline $\mathrm{Ni} 2$ & $\mathrm{P} 2$ & $\mathrm{C} 1 \mathrm{C} 2$ & $16.8(2)$ & $\mathrm{Ni3}$ & N4 & $\mathrm{C} 63 \mathrm{Ni} 4^{2}$ & $-169.59(18)$ \\
\hline $\mathrm{Ni} 2$ & $\mathrm{P} 2$ & C1 C6 & $-165.61(17)$ & $\mathrm{Ni3}$ & N4 & C63 C57 & $11.2(4)$ \\
\hline $\mathrm{Ni} 2$ & $\mathrm{P} 2$ & C8 C9 & $-142.10(19)$ & $\mathrm{Ni3}$ & C39 & $\mathrm{C} 40 \mathrm{C} 1 \mathrm{AA}$ & $0.2(5)$ \\
\hline $\mathrm{Ni} 2$ & $\mathrm{P} 2$ & C8 C13 & $37.4(2)$ & Ni3 & C39 & $\mathrm{C} 40 \mathrm{C} 41$ & 178.9(3) \\
\hline $\mathrm{Ni} 2$ & $\mathrm{P} 2$ & C14C15 & $-116.0(2)$ & $\mathrm{Ni} 4$ & $\mathrm{P} 4$ & C41 C40 & $-19.8(2)$ \\
\hline $\mathrm{Ni} 2$ & $\mathrm{P} 2$ & $\mathrm{C} 14 \mathrm{C} 19$ & $57.3(2)$ & $\mathrm{Ni} 4$ & $\mathrm{P} 4$ & $\mathrm{C} 41 \mathrm{C} 42$ & $161.57(19)$ \\
\hline $\mathrm{Ni} 2$ & N1 & C7 Ni1 & $174.6(2)$ & $\mathrm{Ni} 4$ & $\mathrm{P} 4$ & C45 C46 & $-67.3(2)$ \\
\hline
\end{tabular}




\begin{tabular}{|c|c|c|c|c|c|c|}
\hline $\mathrm{Ni} 2$ & $\mathrm{~N} 1 \quad \mathrm{C} 7 \mathrm{C} 2$ & $-3.5(4)$ & $\mathrm{Ni} 4$ & $\mathrm{P} 4$ & C45 C50 & 105.02(19) \\
\hline $\mathrm{Ni} 2^{1}$ & ${ }^{1} \mathrm{~N} 2 \mathrm{C} 26 \mathrm{C} 21$ & $177.8(3)$ & $\mathrm{Ni} 4$ & $\mathrm{P} 4$ & C51 C52 & $-29.8(2)$ \\
\hline P1 & Ni1 N1 Ni2 - & $-149.5(2)$ & $\mathrm{Ni} 4$ & $\mathrm{P} 4$ & C51 C56 & $150.54(17)$ \\
\hline $\mathrm{P} 1$ & Ni1 N1 C7 2 & $20.3(2)$ & $\mathrm{Ni} 4$ & N3 & C39Ni3 & $-168.55(18)$ \\
\hline P1 & $\mathrm{Ni} 1 \mathrm{~N} 2 \mathrm{Ni} 2^{1} 1$ & $157.6(3)$ & $\mathrm{Ni} 4$ & N3 & C39C40 & $8.0(4)$ \\
\hline P1 & Ni1 N2 C26- & $-10.1(2)$ & $\mathrm{Ni}^{2}$ & N4 & C63C57 & $-179.2(3)$ \\
\hline P1 & Ni1 C7 N1 - & $-170.38(11)$ & P3 & $\mathrm{Ni3}$ & N3 Ni4 & $139.54(18)$ \\
\hline P1 & Ni1 C7 C2 & $6.6(4)$ & $\mathrm{P} 3$ & Ni3 & N3 C39 & $-19.7(2)$ \\
\hline $\mathrm{P} 1$ & C20C21 C22 & $179.30(18)$ & P3 & $\mathrm{Ni3}$ & C39N3 & $170.95(10)$ \\
\hline $\mathrm{P} 1$ & $\mathrm{C} 20 \mathrm{C} 21 \mathrm{C} 263$ & $3.7(3)$ & P3 & $\mathrm{Ni3}$ & C39C40 & $-3.2(4)$ \\
\hline P1 & $\mathrm{C} 20 \mathrm{C} 25 \mathrm{C} 24-$ & $-178.1(2)$ & P3 & C58 & C59C60 & 178.41(18) \\
\hline P1 & C27C28C29 - & $-179.8(2)$ & P3 & C64 & C65 C66 & 179.84(17) \\
\hline P1 & C27C32C31 & 179.99(19) & P3 & C64 & C69 C68 & $-179.73(16)$ \\
\hline $\mathrm{P} 1$ & C33C34C35 & $178.80(19)$ & $\mathrm{P} 3$ & $\mathrm{C} 70$ & C71 C72 & $179.8(2)$ \\
\hline P1 & C33C38C37 - & $-177.4(2)$ & $\mathrm{P} 3$ & $\mathrm{C} 70$ & C75 C74 & $-179.7(2)$ \\
\hline $\mathrm{P} 2$ & Ni2 N1 Ni1 & 179.7(3) & $\mathrm{P} 4$ & $\mathrm{C} 41$ & $\mathrm{C} 42 \mathrm{C} 43$ & $177.8(3)$ \\
\hline $\mathrm{P} 2$ & Ni2 N1 C7 1 & $12.8(2)$ & $\mathrm{P} 4$ & $\mathrm{C} 45$ & C46C47 & $173.0(2)$ \\
\hline $\mathrm{P} 2$ & $\begin{array}{llll}\mathrm{C} 1 & \mathrm{C} 2 & \mathrm{C} 3 & 1\end{array}$ & $175.52(16)$ & $\mathrm{P} 4$ & $\mathrm{C} 45$ & C50C49 & $-171.8(2)$ \\
\hline $\mathrm{P} 2$ & $\mathrm{C} 1 \mathrm{C} 2 \mathrm{C} 7-$ & $-5.8(3)$ & $\mathrm{P} 4$ & C51 & C52C53 & $-179.5(2)$ \\
\hline $\mathrm{P} 2$ & $\mathrm{C} 1 \mathrm{C} 6 \mathrm{C} 5$ & $-176.1(2)$ & $\mathrm{P} 4$ & C51 & C56C55 & 179.86(19) \\
\hline $\mathrm{P} 2$ & C8 C9 C10 & 178.80(19) & N3 & Ni3 & C39C40 & $-174.1(5)$ \\
\hline $\mathrm{P} 2$ & C8 C13C12 - & $-178.9(2)$ & N3 & C39 & C40C1AA & $-172.2(3)$ \\
\hline $\mathrm{P} 2$ & C14C15C16 & $173.2(3)$ & N3 & C39 & C40C41 & $6.5(4)$ \\
\hline $\mathrm{P} 2$ & C14C19C18 - & $-174.6(3)$ & N4 & $\mathrm{Ni3}$ & N3 Ni4 & $-17.9(3)$ \\
\hline N1 & Ni1 N2 Ni2 $2^{1}$ & $-14.4(3)$ & N4 & $\mathrm{Ni3}$ & N3 C39 & $-177.19(13)$ \\
\hline N1 & Ni1 N2 C26 1 & $177.8(2)$ & N4 & Ni3 & C39N3 & $5.3(2)$ \\
\hline N1 & Ni1 C7 C2 & $176.9(5)$ & N4 & $\mathrm{Ni3}$ & C39 C40 & $-168.8(3)$ \\
\hline
\end{tabular}




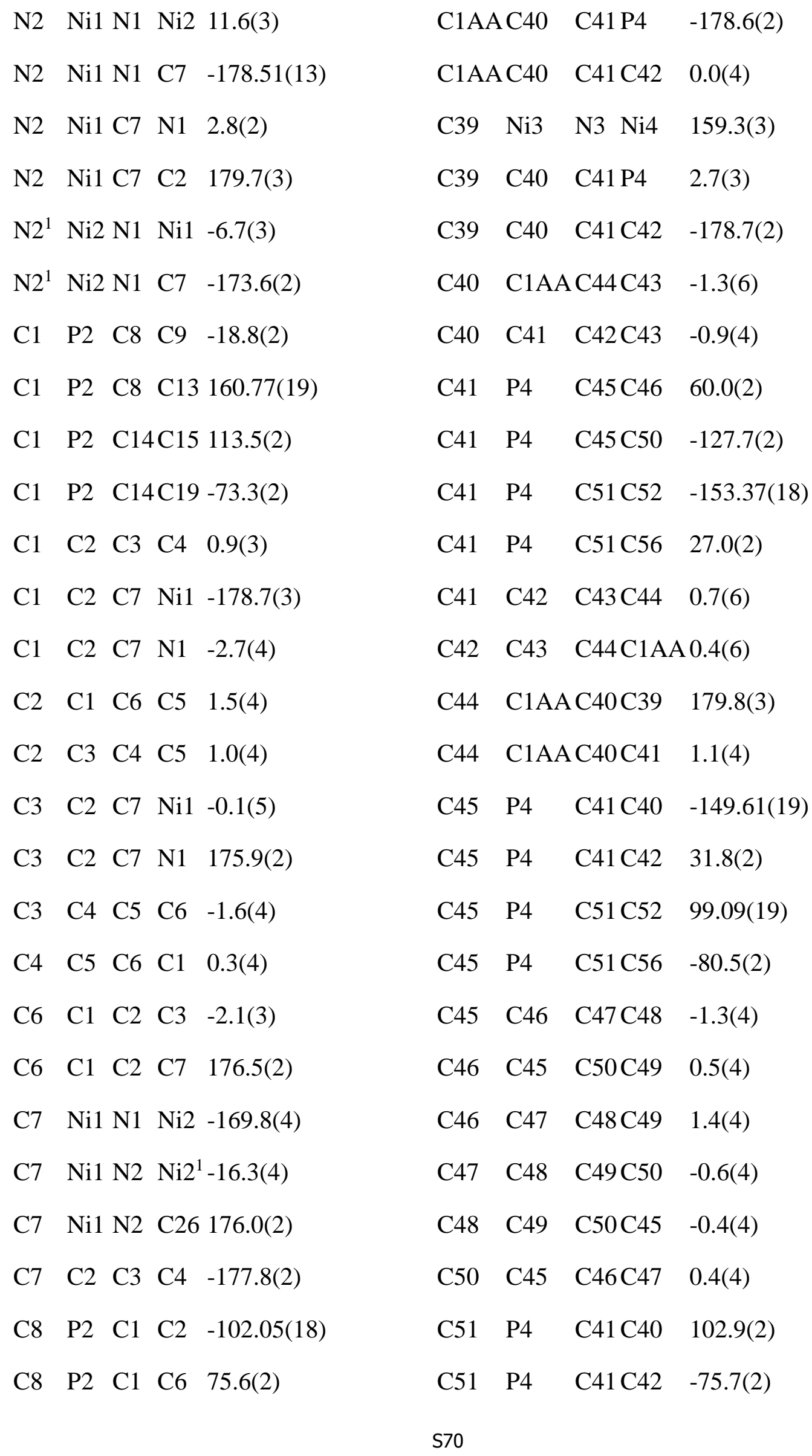




\begin{tabular}{|c|c|c|c|c|c|}
\hline $\mathrm{C} 8$ & P2 C14C15 5.8(2) & C51 & $\mathrm{P} 4$ & $\mathrm{C} 45 \mathrm{C} 46$ & $167.09(19)$ \\
\hline $\mathrm{C} 8$ & P2 C14C19 179.0(2) & C51 & $\mathrm{P} 4$ & C45 C50 & $-20.6(2)$ \\
\hline $\mathrm{C} 8$ & C9 C10C11 0.2(4) & C51 & C52 & $\mathrm{C} 53 \mathrm{C} 54$ & $-0.1(4)$ \\
\hline C9 & C8 C13C12 0.6(4) & C52 & C51 & C56C55 & $0.2(4)$ \\
\hline C9 & C10C11 C12 0.5(4) & C52 & C53 & C54C55 & $-0.2(4)$ \\
\hline $\mathrm{C} 10$ & C11 C12C13 -0.5(5) & C53 & $\mathrm{C} 54$ & C55C56 & $0.6(4)$ \\
\hline $\mathrm{C} 11$ & $\mathrm{C} 12 \mathrm{C} 13 \mathrm{C} 8 \quad 0.0(5)$ & C54 & C55 & C56C51 & $-0.6(4)$ \\
\hline $\mathrm{C} 13$ & C8 C9 C10 -0.7(4) & C56 & C51 & C52 C53 & $0.1(4)$ \\
\hline $\mathrm{C} 14$ & P2 C1 C2 150.00(18) & C57 & C58 & C59C60 & $-0.5(3)$ \\
\hline $\mathrm{C} 14$ & P2 C1 C6 $\quad-32.4(2)$ & C58 & $\mathrm{P} 3$ & C64C65 & $-15.9(2)$ \\
\hline $\mathrm{C} 14$ & P2 $\quad$ C8 $\quad$ C9 $89.0(2)$ & C58 & P3 & C64C69 & $163.73(16)$ \\
\hline $\mathrm{C} 14$ & P2 C8 C13 $-91.5(2)$ & C58 & $\mathrm{P} 3$ & C70C71 & $83.9(2)$ \\
\hline $\mathrm{C} 14$ & C15C16C17 1.8(6) & C58 & $\mathrm{P} 3$ & $\mathrm{C} 70 \mathrm{C} 75$ & $-97.06(19)$ \\
\hline $\mathrm{C} 15$ & C14C19C18 -1.1(5) & C58 & C57 & C62 C61 & $0.7(3)$ \\
\hline $\mathrm{C} 15$ & C16C17C18 -2.3(6) & C58 & C57 & $\mathrm{C} 63 \mathrm{Ni}^{2}$ & $160.5(3)$ \\
\hline $\mathrm{C} 16$ & C17C18C19 1.1(6) & C58 & C57 & C63 N4 & $-21.3(4)$ \\
\hline $\mathrm{C} 17$ & C18C19C14 0.7(6) & C58 & C59 & C60C61 & $0.0(4)$ \\
\hline C19 & C14C15C16 -0.1(5) & C59 & C60 & C61 C62 & $0.9(4)$ \\
\hline $\mathrm{C} 20$ & P1 C27C28 10.0(2) & C60 & C61 & C62 C57 & $-1.2(4)$ \\
\hline $\mathrm{C} 20$ & P1 C27C32 -168.92(17) & C62 & C57 & C58 P3 & $-178.82(16)$ \\
\hline $\mathrm{C} 20$ & P1 C33 C34 -97.2(2) & C62 & C57 & C58C59 & $0.1(3)$ \\
\hline $\mathrm{C} 20$ & P1 C33C38 80.9(2) & C62 & C57 & $\mathrm{C} 63 \mathrm{Ni}^{2}$ & $-22.5(5)$ \\
\hline $\mathrm{C} 20$ & C21 C22C23 -1.7(4) & C62 & C57 & C63 N4 & $155.7(2)$ \\
\hline $\mathrm{C} 20$ & $\mathrm{C} 21 \mathrm{C} 26 \mathrm{Ni} 2^{1}-171.9(3)$ & C63 & C57 & C58P3 & $-1.8(3)$ \\
\hline $\mathrm{C} 20$ & C21 C26N2 13.0(4) & C63 & C57 & C58C59 & $177.16(19)$ \\
\hline $\mathrm{C} 21$ & C20C25C24 0.6(4) & C63 & C57 & C62 C61 & $-176.3(2)$ \\
\hline $\mathrm{C} 21$ & $\mathrm{C} 22 \mathrm{C} 23 \mathrm{C} 24$ 1.7(4) & C64 & P3 & C58C57 & $-90.38(17)$ \\
\hline
\end{tabular}




\begin{tabular}{|c|c|c|c|c|}
\hline $\mathrm{C} 22 \mathrm{C} 21 \mathrm{C} 26 \mathrm{Ni} 2^{1} 12.5(5)$ & C64 & P3 & C58C59 & $90.71(19)$ \\
\hline C22 C21C26N2 -162.7(3) & C64 & $\mathrm{P} 3$ & C70C71 & $-24.1(2)$ \\
\hline C22 C23C24C25 -0.4(4) & C64 & P3 & $\mathrm{C} 70 \mathrm{C} 75$ & $154.96(18)$ \\
\hline C23 C24C25C20 -0.7(4) & C64 & $\mathrm{C} 65$ & C66 C67 & $-0.3(3)$ \\
\hline C25 C20C21 C22 0.6(3) & C65 & C64 & C69C68 & $0.0(3)$ \\
\hline C25 C20C21 C26 -175.0(2) & C65 & C66 & C67 C68 & $0.3(4)$ \\
\hline $\mathrm{C} 26^{1} \mathrm{Ni} 2 \mathrm{~N} 1 \mathrm{Ni} 1 \quad-5.0(4)$ & C66 & C67 & C68 C69 & $-0.2(3)$ \\
\hline $\mathrm{C} 26^{1} \mathrm{Ni} 2 \mathrm{~N} 1 \mathrm{C} 7 \quad-171.9(2)$ & C67 & C68 & C69C64 & $0.1(3)$ \\
\hline C26 C21 C22C23 174.0(2) & C69 & C64 & C65 C66 & $0.2(3)$ \\
\hline C27 P1 C20C21 98.71(18) & $\mathrm{C} 70$ & P3 & C58C57 & $162.02(16)$ \\
\hline C27 P1 C20C25 -82.6(2) & $\mathrm{C} 70$ & P3 & C58C59 & $-16.9(2)$ \\
\hline C27 P1 C33C34 10.1(2) & $\mathrm{C} 70$ & $\mathrm{P} 3$ & C64C65 & $91.51(19)$ \\
\hline C27 P1 C33C38 -171.74(19) & $\mathrm{C} 70$ & $\mathrm{P} 3$ & C64 C69 & $-88.82(17)$ \\
\hline C27 C28C29C30 0.4(5) & $\mathrm{C} 70$ & C71 & $\mathrm{C} 72 \mathrm{C} 73$ & $-0.3(4)$ \\
\hline C28 C27C32C31 1.0(3) & C71 & $\mathrm{C} 70$ & C75 C74 & $-0.6(4)$ \\
\hline C28 C29C30C31 0.0(5) & C71 & $\mathrm{C} 72$ & C73 C74 & $-0.5(5)$ \\
\hline C29 C30C31 C32 0.2(4) & $\mathrm{C} 72$ & $\mathrm{C} 73$ & C74C75 & $0.7(4)$ \\
\hline C30 C31 C32C27 -0.7(4) & C73 & C74 & $\mathrm{C} 75 \mathrm{C} 70$ & $-0.1(4)$ \\
\hline C32 C27C28C29 -0.8(4) & $\mathrm{C} 75$ & $\mathrm{C} 70$ & $\mathrm{C} 71 \mathrm{C} 72$ & $0.8(4)$ \\
\hline C33 P1 C20C21 -153.54(18) & $\mathrm{C} 78$ & N5 & $\mathrm{C} 76 \mathrm{O} 1$ & $176.2(5)$ \\
\hline C33 P1 C20C25 25.1(2) & $\mathrm{C} 78$ & N5 & C76C77 & $-5.6(8)$ \\
\hline C33 P1 C27C28 -96.1(2) & C79 & N5 & $\mathrm{C} 76 \mathrm{O} 1$ & $3.2(8)$ \\
\hline C33 P1 C27C32 84.94(18) & $\mathrm{C} 79$ & N5 & C76C77 & $-178.7(4)$ \\
\hline C33 C34C35C36 -1.5(4) & $\mathrm{C} 82$ & N6 & $\mathrm{C} 80 \mathrm{O} 2$ & $-174.2(6)$ \\
\hline C34 C33 C38C37 0.8(4) & $\mathrm{C} 82$ & N6 & $\mathrm{C} 80 \mathrm{C} 81$ & $3.5(8)$ \\
\hline C34 C35C36C37 0.7(4) & $\mathrm{C} 83$ & N6 & $\mathrm{C} 80 \mathrm{O} 2$ & $8.5(7)$ \\
\hline C35 C36C37 C38 0.8(4) & $\mathrm{C} 83$ & N6 & C80C81 & $-173.8(5)$ \\
\hline
\end{tabular}


${ }^{1} 2-X,-Y,-Z ;{ }^{2} 1-X,-1-Y, 1-Z$

Table 7 Hydrogen Atom Coordinates $\left(\AA \times 10^{4}\right)$ and Isotropic Displacement Parameters $\left(\AA^{2} \times 10^{3}\right)$ for shuxz_1105_3.

\begin{tabular}{|c|c|c|c|c|}
\hline Atom & $x$ & $y$ & $z$ & $\mathbf{U}(\mathbf{e q})$ \\
\hline H3 & 9211.63 & -421.03 & 2431.62 & 35 \\
\hline $\mathrm{H} 4$ & 9198.79 & -1312.06 & 3238.63 & 44 \\
\hline H5 & 9538.24 & -2673.47 & 3159.09 & 47 \\
\hline H6 & 9817.89 & -3189.43 & 2248.69 & 41 \\
\hline H9 & 7954.01 & -3931.15 & 1466.31 & 43 \\
\hline H10 & 6330.87 & -5232.8 & 979.38 & 54 \\
\hline H11 & 5910.92 & -5723.35 & -67.1 & 61 \\
\hline H12 & 7125.46 & -4924.4 & -632.12 & 65 \\
\hline H13 & 8746.41 & -3622.51 & -153.55 & 51 \\
\hline H15 & 9635.18 & -4584.78 & 956.57 & 63 \\
\hline H16 & 10812.8 & -5255.37 & 1149.49 & 84 \\
\hline H17 & 12536.48 & -4272.53 & 1619.61 & 78 \\
\hline H18 & 13148.99 & -2587.04 & 1844.18 & 86 \\
\hline H19 & 11985.72 & -1885.16 & 1620.91 & 72 \\
\hline $\mathrm{H} 22$ & 8739.58 & 3150.59 & -120.18 & 41 \\
\hline $\mathrm{H} 23$ & 7955.63 & 3945.86 & 348.4 & 50 \\
\hline $\mathrm{H} 24$ & 7804.67 & 3753.41 & 1347.64 & 48 \\
\hline $\mathrm{H} 25$ & 8409.43 & 2753.32 & 1867.2 & 38 \\
\hline $\mathrm{H} 28$ & 10672.51 & 3640.64 & 1962.28 & 48 \\
\hline $\mathrm{H} 29$ & 12347.24 & 4630.84 & 2577.41 & 62 \\
\hline H30 & 13371.68 & 3922.46 & 3057.7 & 55 \\
\hline H31 & 12715.21 & 2217.6 & 2925.21 & 50 \\
\hline
\end{tabular}




\begin{tabular}{|c|c|c|c|}
\hline H32 & 11051.27 & 1219.2 & 2310.67 \\
\hline H34 & 9533.43 & 2087.98 & 2901.11 \\
\hline H35 & 8382.32 & 1577.64 & 3547.54 \\
\hline H36 & 6680.89 & 359.08 & 3173.85 \\
\hline H37 & 6113.49 & -389.22 & 2157.72 \\
\hline H38 & 7266.83 & 59.17 & 1523.12 \\
\hline $\mathrm{H} 1 \mathrm{~A} A$ & 6478.63 & -4907.75 & 2782.34 \\
\hline $\mathrm{H} 42$ & 6511.53 & -7885.87 & 3164.71 \\
\hline $\mathrm{H} 43$ & 7173.5 & -7133.34 & 2373.42 \\
\hline $\mathrm{H} 44$ & 7149.29 & -5652.34 & 2177.1 \\
\hline H46 & 7672.28 & -6911.98 & 4544.62 \\
\hline $\mathrm{H} 47$ & 8690.89 & -7660.04 & 4979.4 \\
\hline $\mathrm{H} 48$ & 7902.88 & -9333 & 5056.35 \\
\hline H49 & 6118.95 & -10263.58 & 4660.94 \\
\hline H50 & 5093.26 & -9539.45 & 4203.11 \\
\hline H52 & 3487.64 & -8602.45 & 4326.38 \\
\hline H53 & 1859.53 & -9883.25 & 3816.08 \\
\hline H54 & 1588.87 & -10565.7 & 2817.54 \\
\hline H55 & 2930.72 & -9969.52 & 2317.93 \\
\hline H56 & 4566.41 & -8705.12 & 2819.19 \\
\hline H59 & 5116.63 & -1933.4 & 2698.54 \\
\hline H60 & 4145.92 & -1066.39 & 2769.24 \\
\hline H61 & 3425.19 & -1095.36 & 3603.71 \\
\hline H62 & 3712.15 & -1958.41 & 4386.13 \\
\hline H65 & 7005.87 & -1045.27 & 3835.85 \\
\hline H66 & 8760 & -17.88 & 4323.19 \\
\hline H67 & 9914.83 & -690.8 & 4618.15 \\
\hline
\end{tabular}




\begin{tabular}{|c|c|c|c|c|}
\hline H68 & 9342.07 & -2385.85 & 4422.06 & 39 \\
\hline H69 & 7588.65 & -3418.43 & 3930.87 & 33 \\
\hline H71 & 7006.28 & -2012.15 & 2645.19 & 43 \\
\hline H72 & 6950.92 & -2225.13 & 1607.63 & 56 \\
\hline H73 & 5685.47 & -3648.11 & 972.71 & 59 \\
\hline H74 & 4472.5 & -4886.78 & 1366.28 & 59 \\
\hline $\mathrm{H} 75$ & 4502.51 & -4690.12 & 2402.31 & 44 \\
\hline H77A & 7445.6 & -2373.17 & 216.18 & 143 \\
\hline H77B & 6596.78 & -2154.57 & 447.65 & 143 \\
\hline $\mathrm{H} 77 \mathrm{C}$ & 7422.32 & -1349.48 & 159.17 & 143 \\
\hline H78A & 7945.67 & -1197.57 & -1087.94 & 227 \\
\hline H78B & 8105.6 & -2160.22 & -1043.37 & 227 \\
\hline $\mathrm{H} 78 \mathrm{C}$ & 8196.33 & -1514.78 & -443.66 & 227 \\
\hline H79A & 5267.17 & -3114.34 & -1586.48 & 171 \\
\hline H79B & 6226.1 & -3148.08 & -1797.31 & 171 \\
\hline H79C & 6070.99 & -2179.73 & -1802.76 & 171 \\
\hline H81A & 9030.13 & -5002.78 & 4395.83 & 223 \\
\hline H81B & 9265.06 & -5729.26 & 3994.99 & 223 \\
\hline H81C & 8424.21 & -5376.94 & 3700.85 & 223 \\
\hline H82A & 9756.04 & -5953.02 & 3345.56 & 371 \\
\hline H82B & 10677.31 & -5513.97 & 3011.78 & 371 \\
\hline H82C & 9562.34 & -5591.81 & 2709.87 & 371 \\
\hline H83A & 11427.25 & -3068.05 & 3501.45 & 204 \\
\hline H83B & 11108.33 & -3641.03 & 2832.85 & 204 \\
\hline H83C & 11881.13 & -3811.48 & 3359.65 & 204 \\
\hline
\end{tabular}




\section{References}

(1) Armarego, W. L. F.; Chai, C. C. L. Purification of laboratory chemicals, 5th ed., ButterworthHeinemann, 2003.

(2) Reis, A.; Dehe, D.; Farsadpour, S.; Munstein, I.; Sun, Y.; Thiel, W. R. Fluoride catalyzed Paryl-coupling-a mild approach to functionalized arylphosphines. New J. Chem., 2011, 35, 2488.

(3) Li, J.; Fu, H.; Hu, P.; Zhang, Z.; Li, X.; Cheng, Y. Catalysts for Suzuki Polycondensation: Ionic and "Quasi-Ionic" Amphipathic Palladium Complexes with Self-Phase-Transfer Features. Chem. Eur. J. 2012, 18, 13941.

(4) Liao, L.; Guo, R.; Zhao, X. Organoselenium-Catalyzed Regioselective C-H Pyridination of 1,3-Dienes and Alkenes. Angew. Chem. Int. Ed. 2017, 56, 3201.

(5) Huang, H.-M.; Koy, M.; Serrano, E.; Pflüger, P. M.; Schwarz, J. L.; F. Glorius. Catalytic radical generation of $\pi$-allylpalladium complexes. Nat. Catal. 2020, 3, 393.

(6) Preu $\beta$, T.; Saak, W.; Doye, S. Titanium-Catalyzed Intermolecular Hydroaminoalkylation of Conjugated Dienes. Chem. Eur. J. 2013, 19, 3833.

(7) Jing, S. M.; Balasanthiran, V.; Pagar, V.; Gallucci, J. C.; RajanBabu, T. V. Catalytic Enantioselective Hetero-dimerization of Acrylates and 1,3-Dienes. J. Am. Chem. Soc. 2017, 139, 18034.

(8) Feng, X.; Sippel, C.; Knaup, F. H.; Bracher, A.; Staibano, S.; Romano, M. F.; Hausch, F. A Novel Decalin-Based Bicyclic Scaffold for FKBP51-Selective Ligands. J. Med. Chem. 2020, 63, 231.

(9) Cheung, K. P. S.; Kurandina, D.; Yata, T.; Gevorgyan, V. Photoinduced Palladium-Catalyzed Carbofunctionalization of Conjugated Dienes Proceeding via Radical-Polar Crossover Scenario: 1,2-Aminoalkylation and Beyond. J. Am. Chem. Soc. 2020, 142, 9932.

(10)Graham, T. J. A.; Poole, T. H.; Reese, C. N.; Goess, B. C. Regioselective Semihydrogenation of Dienes. J. Org. Chem. 2011, 76, 4132.

(11)Miller, D. J.; Yu, F.; Young, N. J.; Allemann, R. K. Competitive inhibition of aristolochene synthase by phenyl-substituted farnesyl diphosphates: evidence of active site plasticity. $\mathrm{Org}$. Biomol. Chem., 2007, 5, 3287.

(12)Fontán, N.; Domínguez, M.; Álvarez, R.; de Lera, Á. R. Synthesis of C40-Symmetrical Fully Conjugated Carotenoids by Olefin Metathesis. Eur. J. Org. Chem. 2011, 6704.

(13)Ji, D.-W.; He, G.-C.; Zhang, W.-S.; Zhao, C.-Y.; Hu, Y.-C.; Chen, Q.-A. Nickel-catalyzed allylallyl coupling reactions between 1,3-dienes and allylboronates. Chem. Commun., 2020, 56, 7431.

(14)Liao, L.; Guo, R.; Zhao, X. Organoselenium-Catalyzed Regioselective C-H Pyridination of 1,3Dienes and Alkenes. Angew.Chem. Int. Ed. 2017, 56, 3201.

(15)Rajkumar, S.; Savarimuthu, S. A.; Kumaran, R. S.; Nagarajad, C. M.; Gandhi, T. Expedient Synthesis of New Cinnolinediones by Ru-Catalyzed Regioselective Unexpected Deoxygenation-Oxidative Annulation of Propargyl Alcohols with Phthalazinones and Pyridazinones. Chem. Commun., 2016, 52, 2509.

(16)Jiang, T.; Huynh, K.; Livinghouse, T. Substrate Structural Effects in Yttrium(III)-Catalyzed Hydroamination/Cyclizations of 1,2-Disubstituted and 1,1,2-Trisubstituted Aminoalkenes -Terminated by 2-(Phenyl) and 2-(2-Heteroarenyl) Groups. Synlett 2013, 24, 193. 
(17) Su, X.-L.; Huang, H.-G.; Yuan, Y.-F.; Li, Y. Radical Desulfur-Fragmentation and Reconstruction of Enol Triflates: Facile Access to a-Trifluoromethyl Ketones. Angew. Chem. Int. Ed. 2017, 56, 1338.

(18)Navendu, J.; Quyen, N.; Tom, G. D. Development of a Suzuki Cross-Coupling Reaction between 2-Azidoarylboronic Pinacolate Esters and Vinyl Triflates To Enable the Synthesis of [2,3]-Fused Indole Heterocycles. J. Org. Chem. 2014, 79, 2781.

(19) Hu, J-T.; Zheng, B.; Chen, Y-C.; Xiao, Q. Expedient Synthesis of 9,10-Phenanthrenes via LiOPiv-Promoted and Palladium-Catalysed Aryne Annulation by Vinyl Triflates. Org. Chem. Front. 2018, 5, 2045.

(20) Li, W.; Yu, S.; Li, J.; Zhao, Y. Nickel-Catalyzed Allylmethylation of Alkynes with Allylic Alcohols and AlMe3: Facile Access to Skipped Dienes and Trienes. Angew. Chem. Int. Ed. 2020, 59, 14404.

(21) Duan, J.-C.; Du, Y-F.; Pang, X.-B.; Shu, X.-Z. Ni-catalyzed Cross-Electrophile Coupling between Vinyl/Aryl and Alkyl Sulfonates: Synthesis of Cycloalkenes and Modification of Peptides. Chem. Sci. 2019, 10, 8706.

(22) Lippincott, D. J.; Linstadt, R. T. H.; Maser, M. R.; Lipshutz, B. H. Synthesis of Functionalized [3], [4], [5] and [6]Dendralenes through Palladium-Catalyzed Cross-Couplings of Substituted Allenoates. Angew. Chem. Int. Ed. 2017, 56, 847.

(23) Kutsumura, N.; Shibuya, K.; Yamaguchi, H.; Saito, T. $n$-Butyllithium-promoted regioselective elimination of vicinal bis-triflate having an adjacent ether oxygen. Tetrahedron Lett. 2017, 58 4099.

(24)Tian, Z.-X.; Qiao, J.-B.; Xu, G.-L.; Pang, X.-B, Qi, L.-L.; Ma, W.-Y, Zhao Z.-Z.; Duan, J.-C.; Du, Y.-F.; Su, P.-F.; Liu, X.-Y.; Shu, X.-Z. Highly Enantioselective Cross-Electrophile ArylAlkenylation of Unactivated Alkenes. J. Am. Chem. Soc. 2019, 141, 7637.

(25)Duan, J.; Wang, K.; Xu, G.-L.; Kang, S.; Qi, L.; Liu, X.-Y.; Shu, X.-Z. Cross-Electrophile Csp²-Si Coupling of Vinyl Chlorosilanes. Angew. Chem. Int. Ed. 2020, 59, 23083.

(26) Cho, E. J.; Buchwald, S. L. The Palladium-Catalyzed Trifluoromethylation of Vinyl Sulfonates. Org. Lett. 2011, 13, 6552.

(27) Koch, V.; Bräse. S. Pd-Mediated Cross-Coupling of C-17 Lithiated and Rost-16-en-3-ol Access to Functionalized Arylated Steroid Derivatives. Org. Biomol. Chem. 2017, 15, 92.

(28) Hu, X.; Wang, G.; Qin, C.; Xie, X.; Zhang, C.; Xu, W.; Liu, Y. Ligandless Nickel-Catalyzed Transfer Hydrogenation of Alkenes and Alkynes Using Water as the Hydrogen Donor. Org. Chem. Front., 2019, 6, 2619. 


\section{Copies of NMR Spectra for Compounds}

L8; ${ }^{1} \mathrm{H}$ NMR (400MHz, $\left.\mathrm{CDCl}_{3}\right) ;{ }^{13} \mathrm{C}$ NMR (100 $\left.\mathrm{MHz}, \mathrm{CDCl}_{3}\right)$

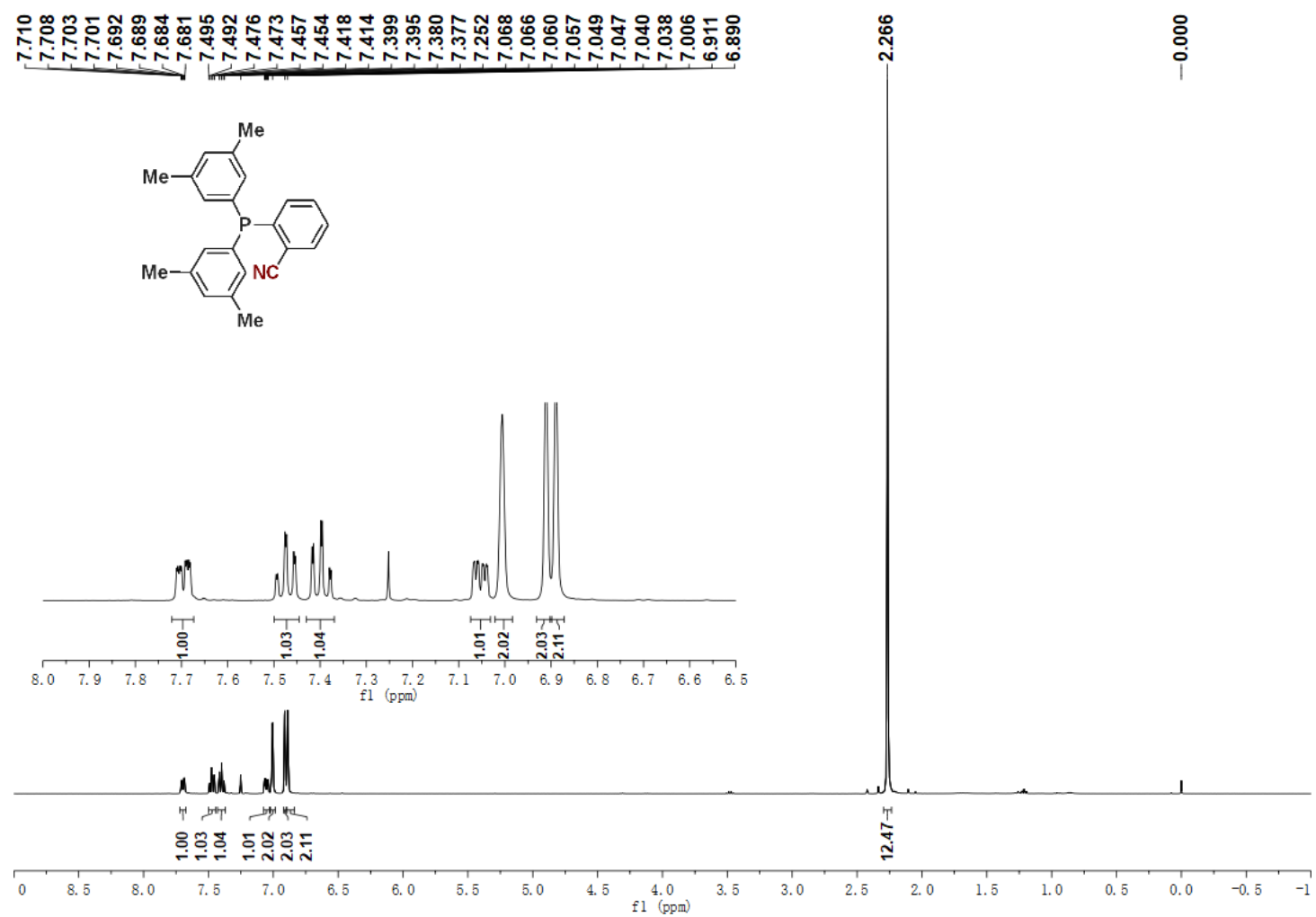

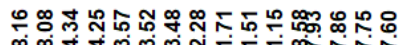

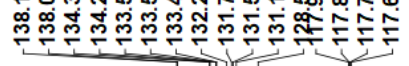

ำ

송

$\stackrel{\text { }}{\grave{i}}$
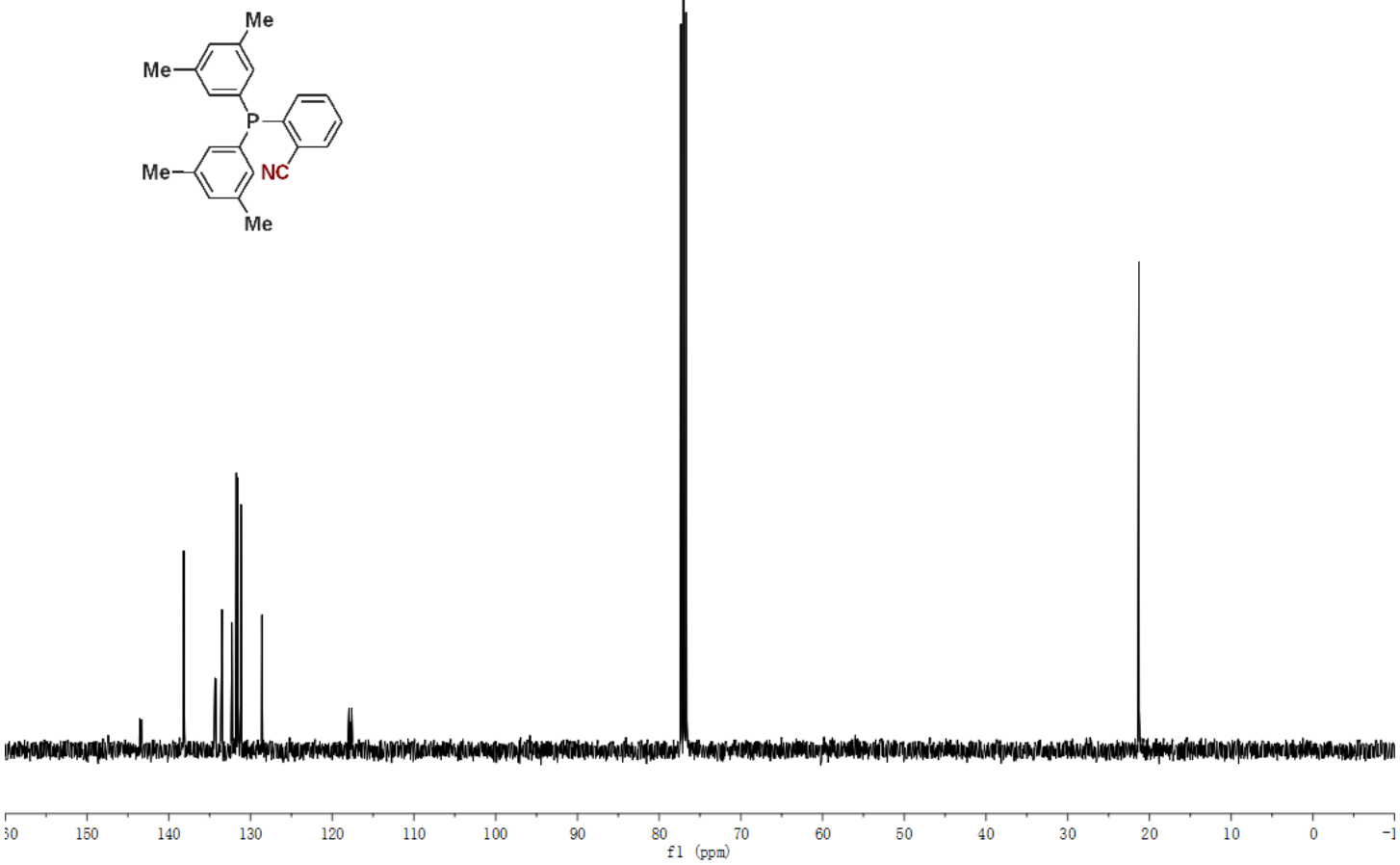

S78 
L8; ${ }^{31}$ P NMR (161 MHz, $\left.\mathrm{CDCl}_{3}\right)$

i
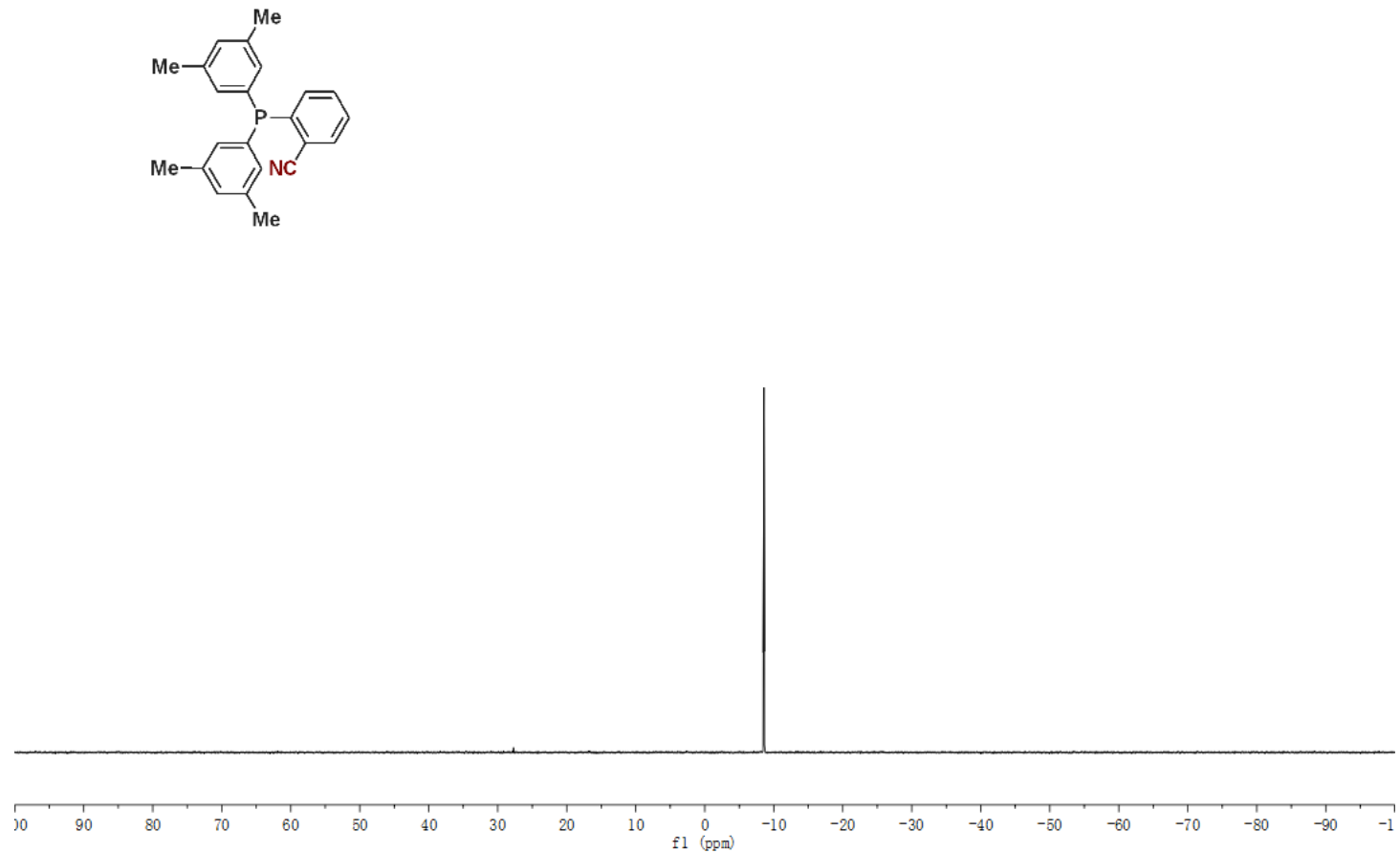
L9; ${ }^{1} \mathrm{H}$ NMR (400MHz, $\left.\mathrm{CDCl}_{3}\right) ;{ }^{13} \mathrm{C}$ NMR (100 MHz, $\left.\mathrm{CDCl}_{3}\right)$
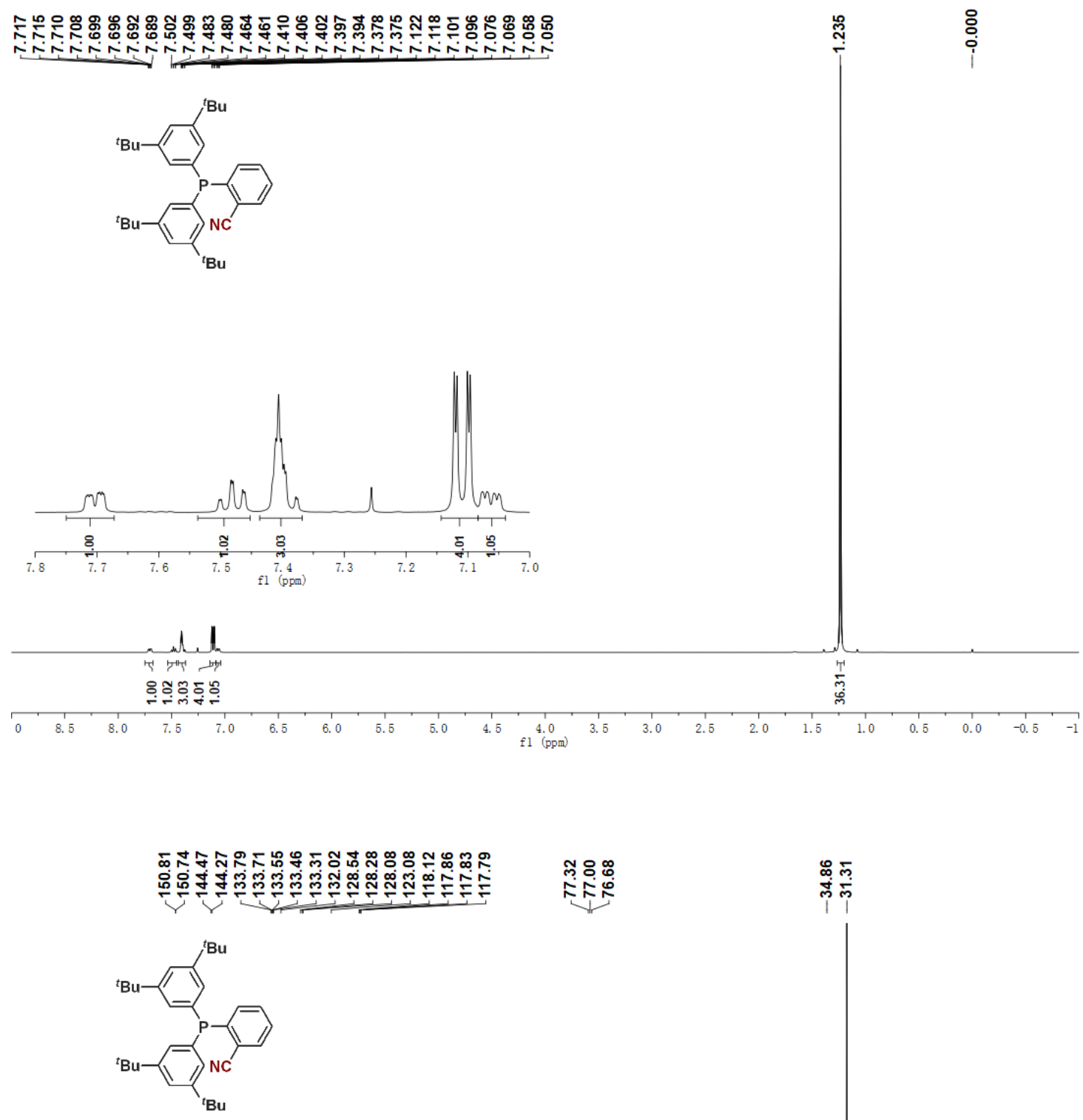

×

年最

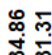

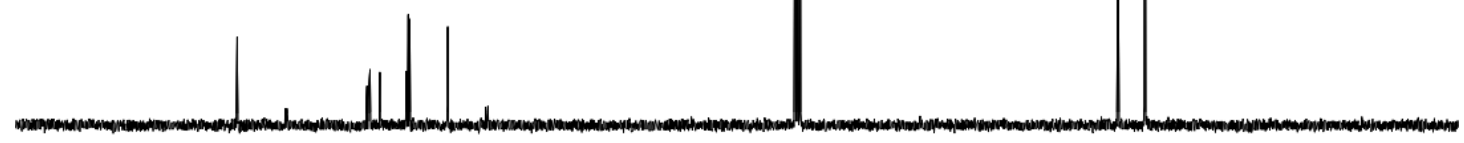

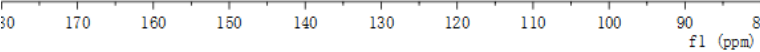


L9; ${ }^{31}$ P NMR (161 MHz, $\left.\mathrm{CDCl}_{3}\right)$

$\stackrel{?}{6}$
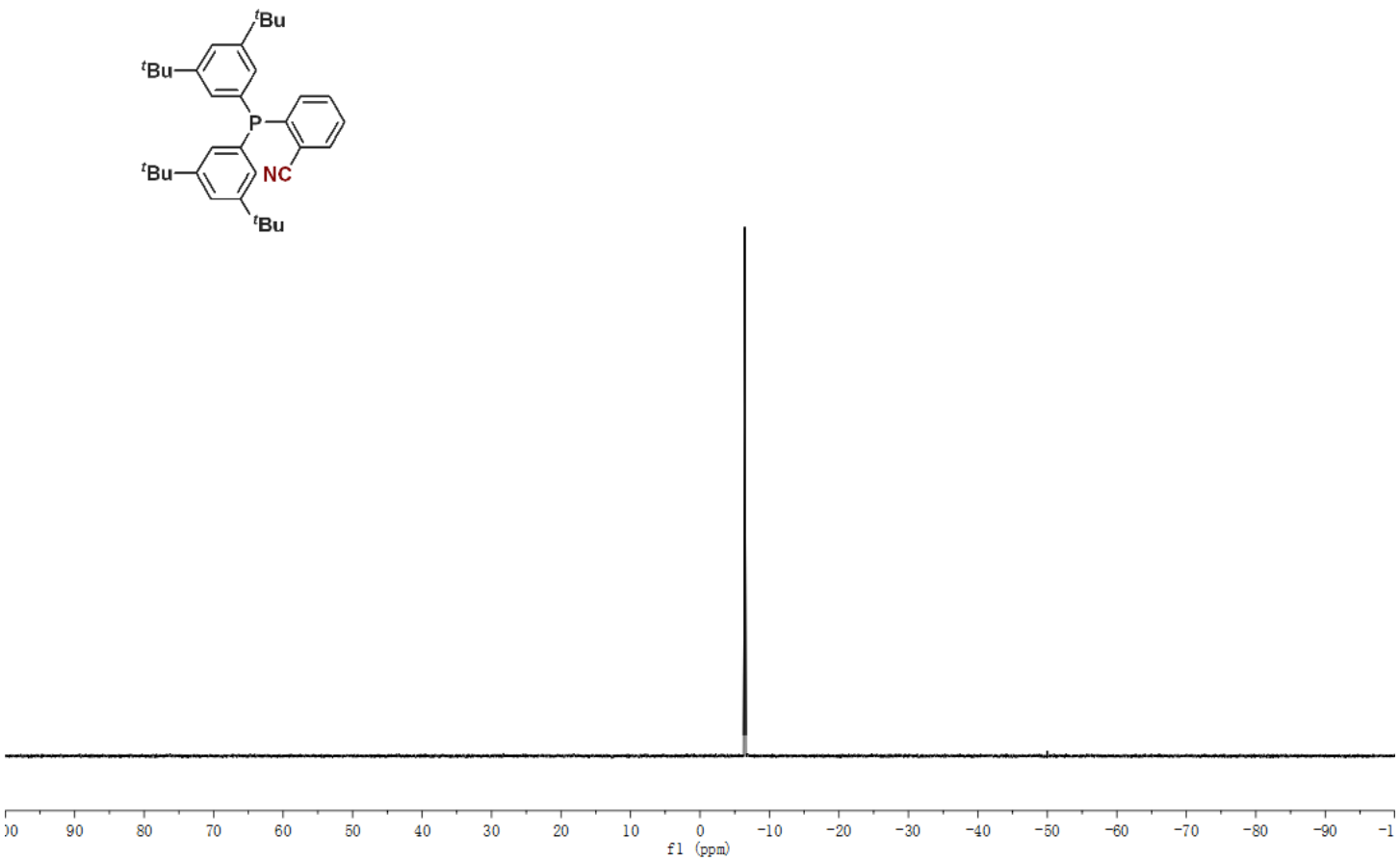
L10; ${ }^{1} \mathrm{H}$ NMR (400MHz, $\left.\mathrm{CDCl}_{3}\right) ;{ }^{13} \mathrm{C}$ NMR $\left(100 \mathrm{MHz}, \mathrm{CDCl}_{3}\right)$

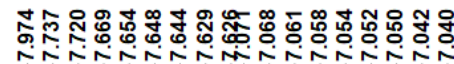
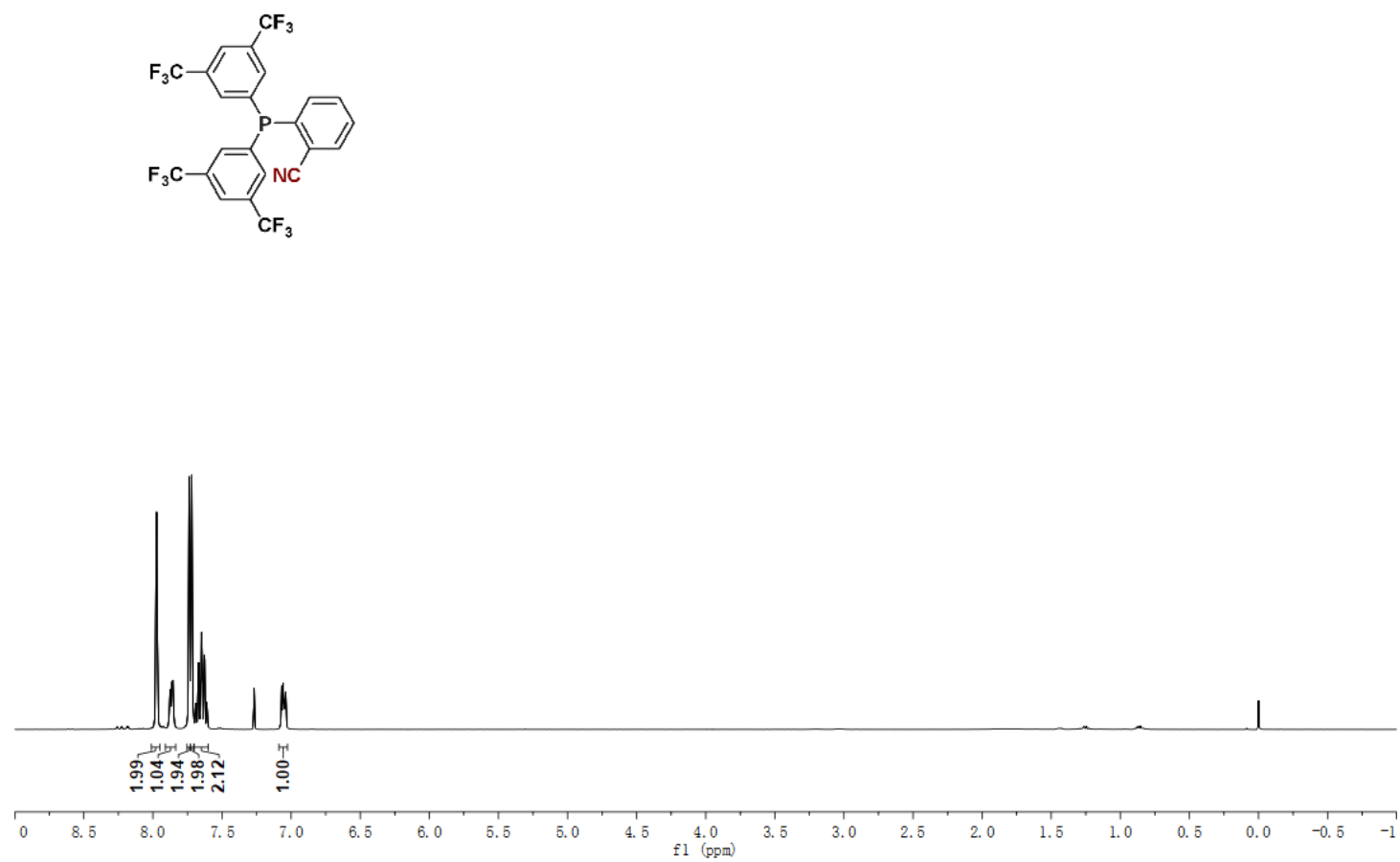

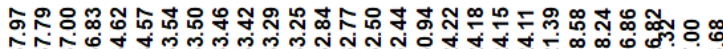

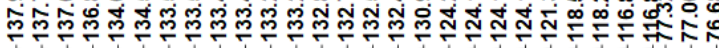

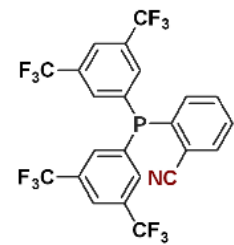

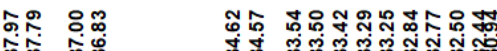

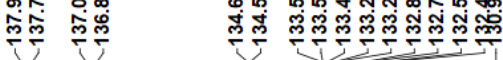

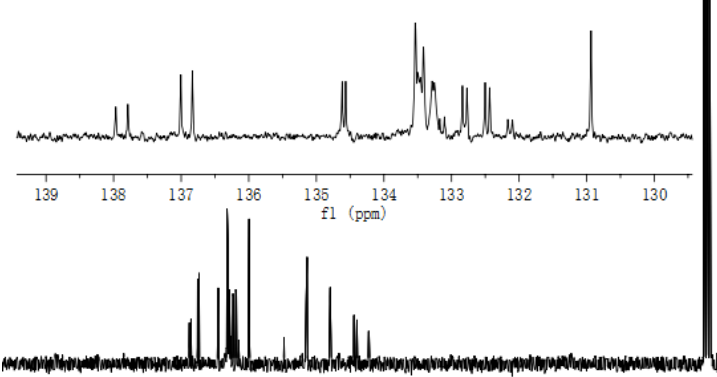
${ }^{80} 11(\mathrm{popm})^{70}$ 
L10; ${ }^{31}$ P NMR (161 MHz, CDCl $) ;{ }^{19}$ F NMR (376 MHz, $\left.\mathrm{CDCl}_{3}\right)$

\section{$\stackrel{0}{\circ}$}
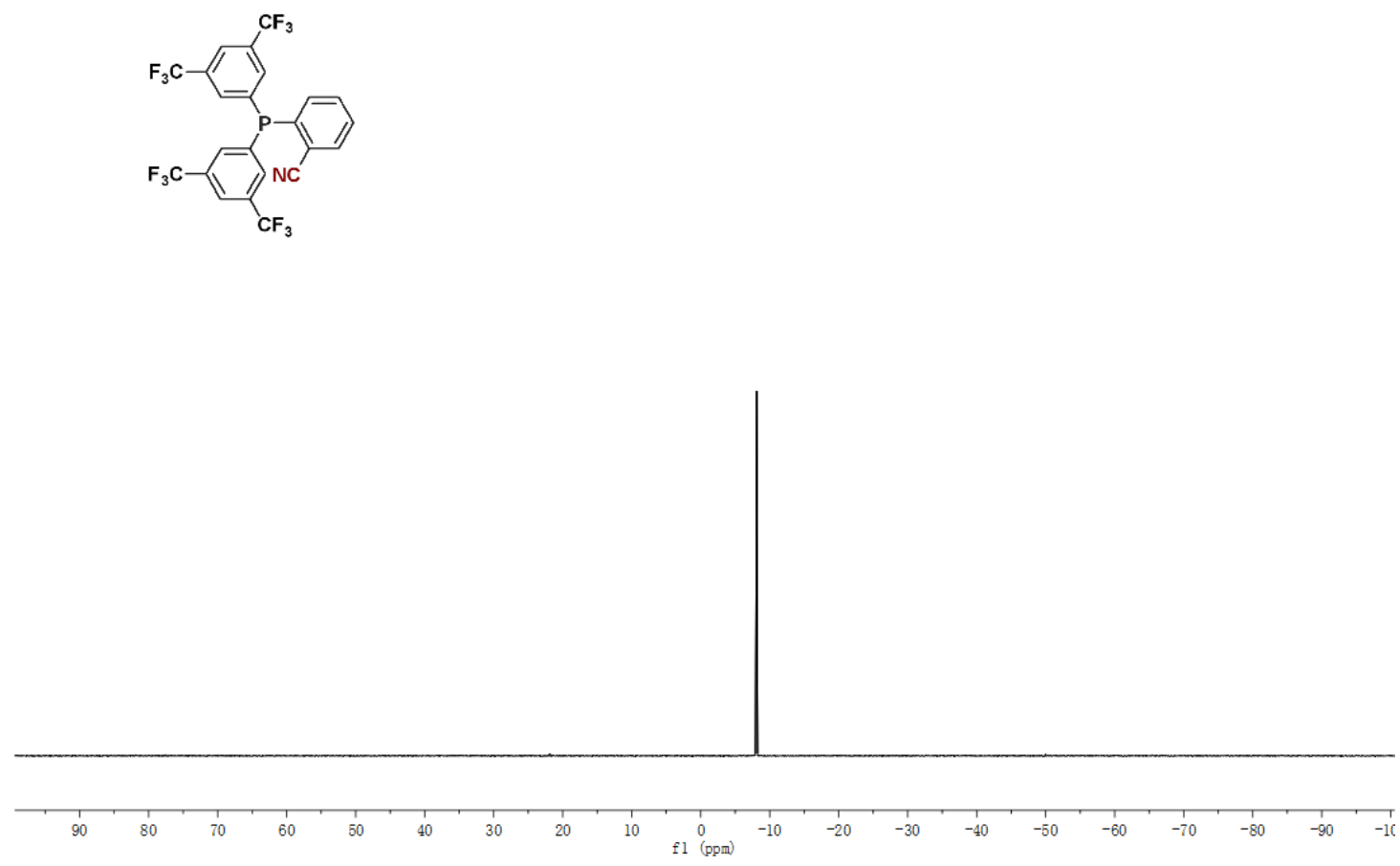

¿̊.
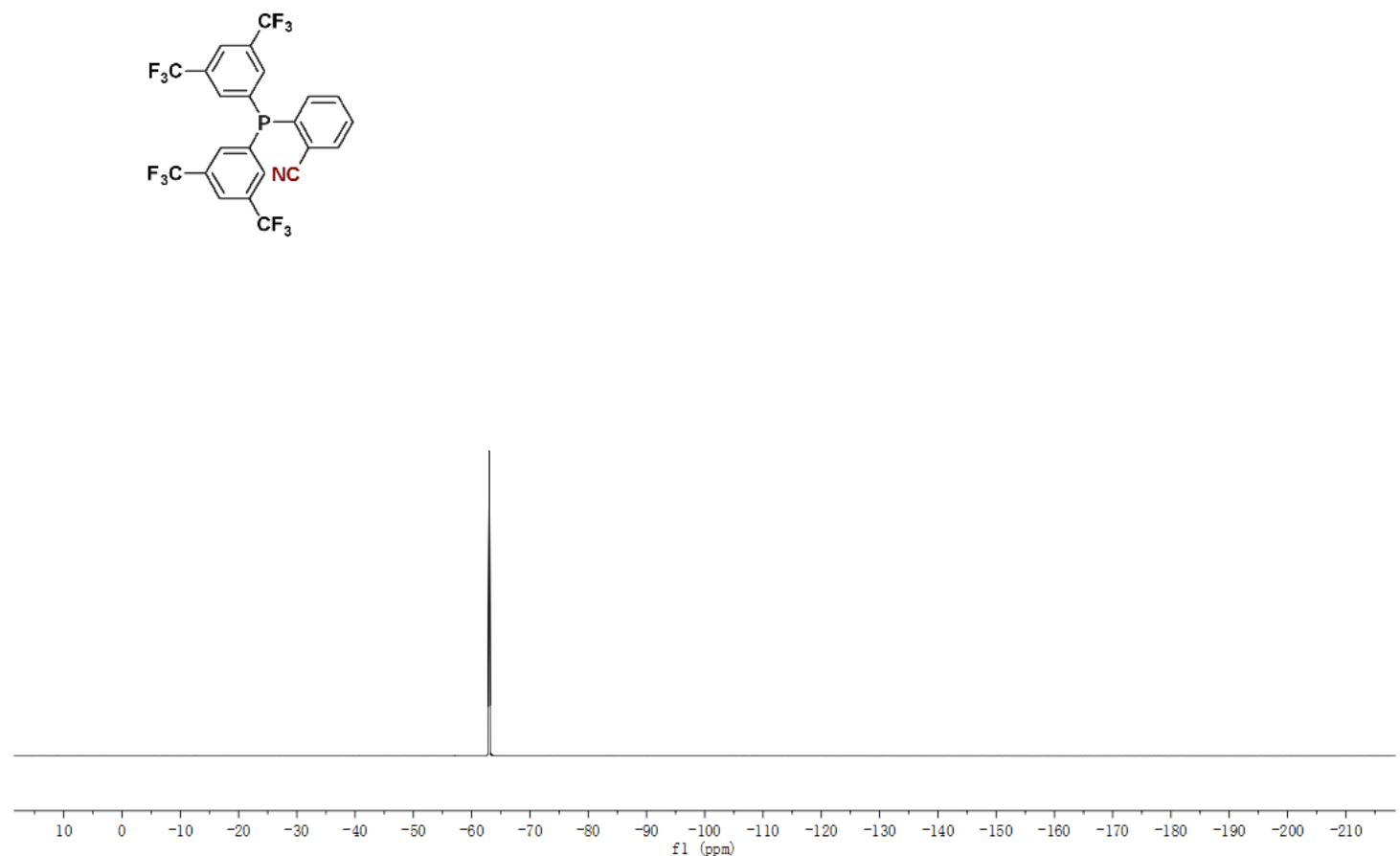
1e; ${ }^{1} \mathrm{H}$ NMR (400MHz, $\left.\mathrm{CDCl}_{3}\right) ;{ }^{13} \mathrm{C}$ NMR (100 MHz, $\left.\mathrm{CDCl}_{3}\right)$

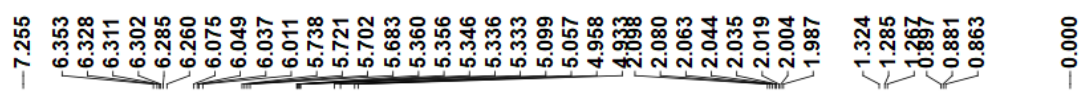

$\mathrm{H}_{17} \mathrm{C}_{8} \mathrm{NH}_{7} \mathrm{~N}$

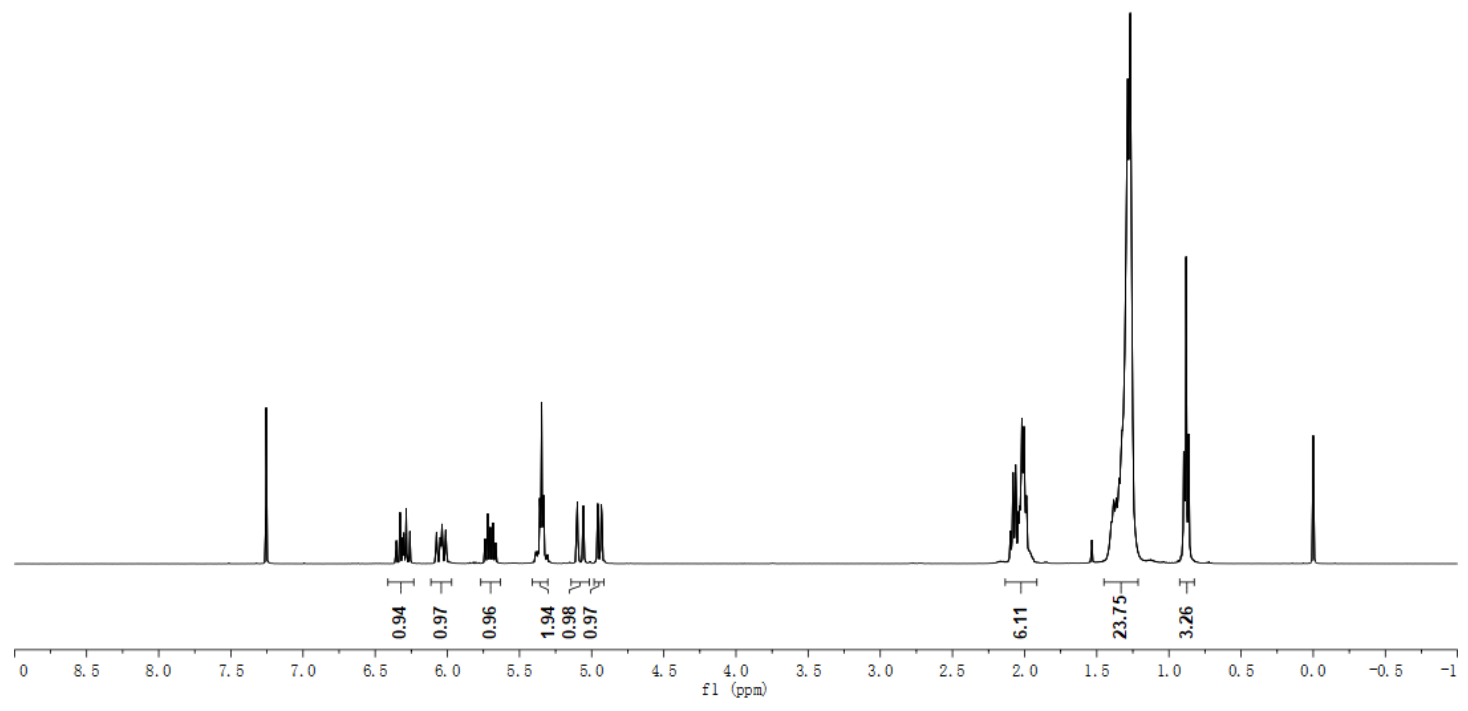

\begin{tabular}{|c|c|c|c|}
\hline 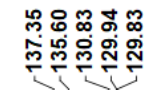 & $\underset{\stackrel{L}{\circ}}{\stackrel{\leftrightarrow}{+}}$ & 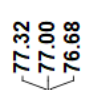 & 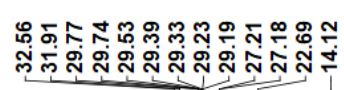 \\
\hline
\end{tabular}

$\mathrm{H}_{17} \mathrm{C}_{8} \sim \mathrm{H}_{7} \curvearrowright$

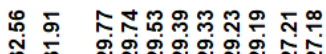

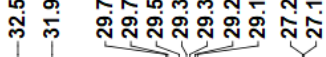

ลั่

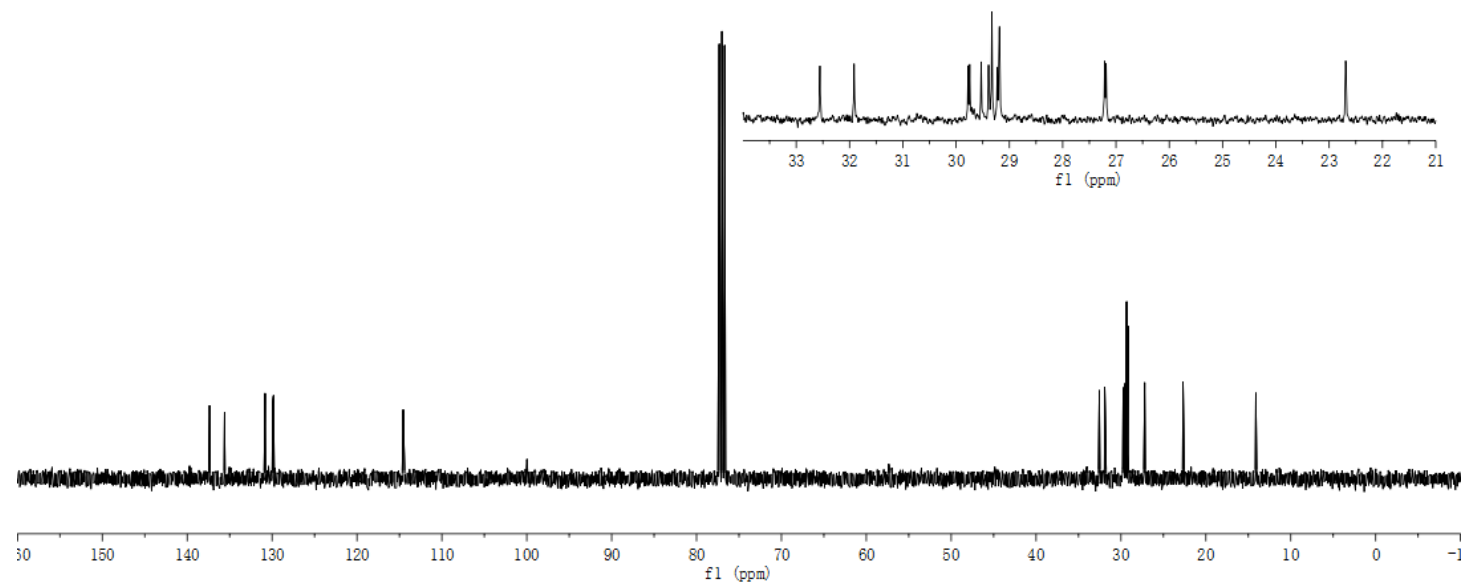


1g; ${ }^{1} \mathrm{H}$ NMR (400MHz, $\left.\mathrm{CDCl}_{3}\right) ;{ }^{13} \mathrm{C}$ NMR $\left(100 \mathrm{MHz}, \mathrm{CDCl}_{3}\right)$

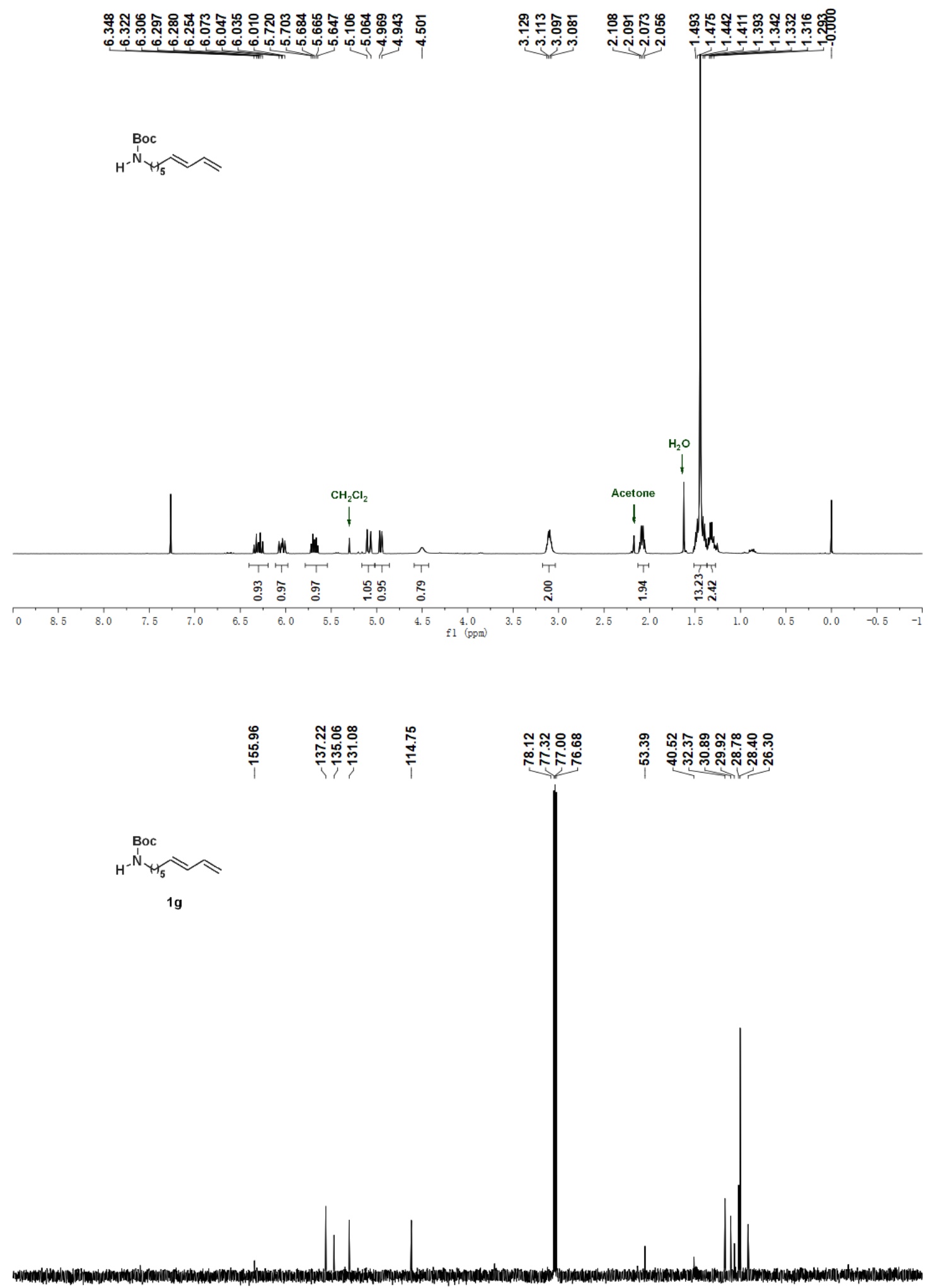

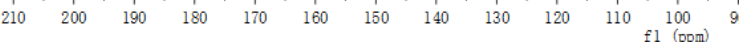


1i; ${ }^{1} \mathrm{H}$ NMR (400MHz, $\left.\mathrm{CDCl}_{3}\right) ;{ }^{13} \mathrm{C}$ NMR (100 MHz, $\left.\mathrm{CDCl}_{3}\right)$

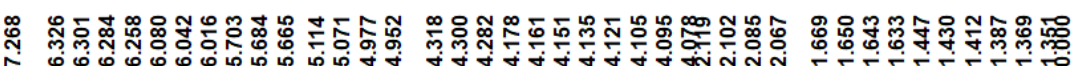

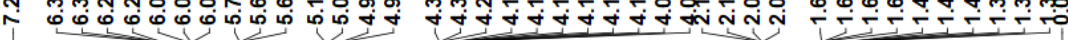

Me $\prod_{0}^{N H B o c}{ }_{0}^{O} H_{5}$

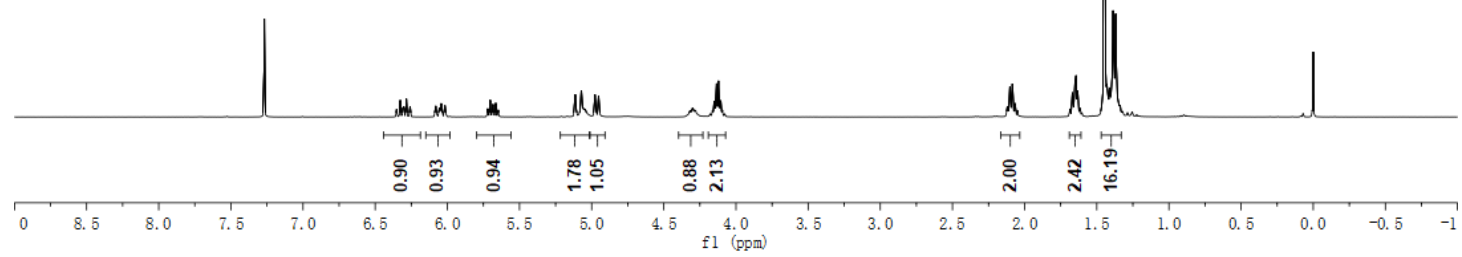

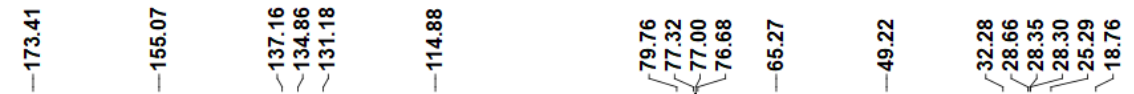

$M e \prod_{0}^{N H B o c}{ }^{0} H_{5} \curvearrowright$

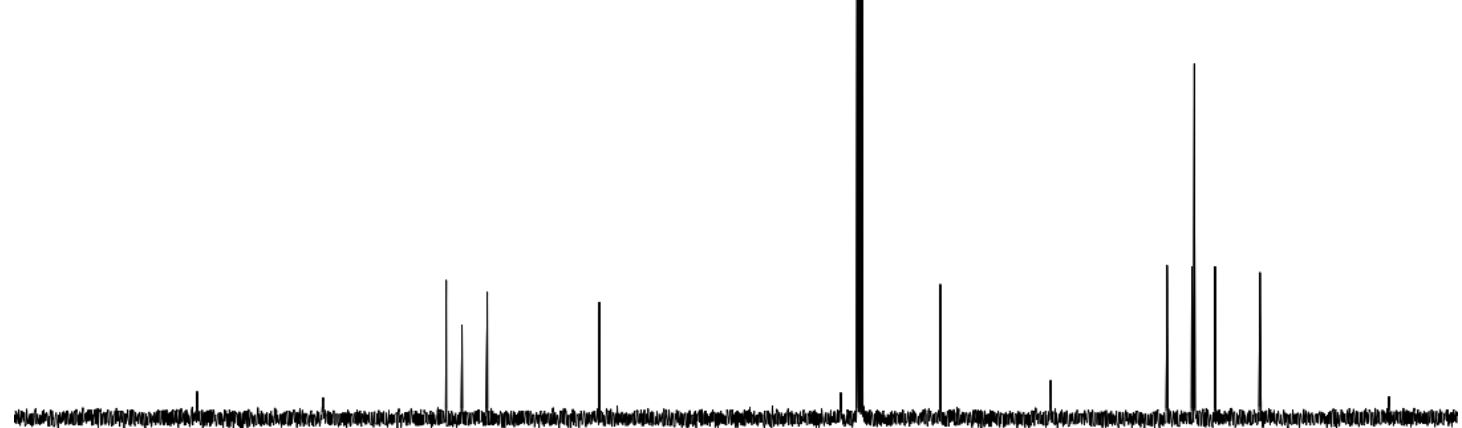

$\begin{array}{lllllllllllllllllllllllllll}190 & 180 & 170 & 160 & 150 & 140 & 130 & 120 & 110 & 100 & 90 & 80 & 70 & 60 & 50 & 40 & 30 & 20 & 10 & 0 & -1\end{array}$ 
1j; ${ }^{1} \mathrm{H}$ NMR (400MHz, $\left.\mathrm{CDCl}_{3}\right) ;{ }^{13} \mathrm{C}$ NMR (100 MHz, $\left.\mathrm{CDCl}_{3}\right)$

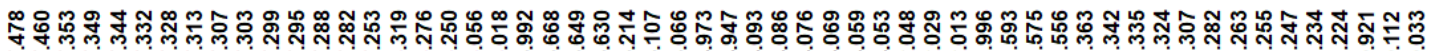

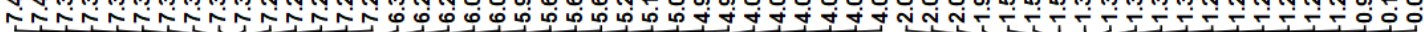

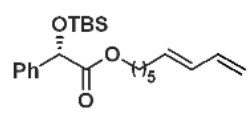

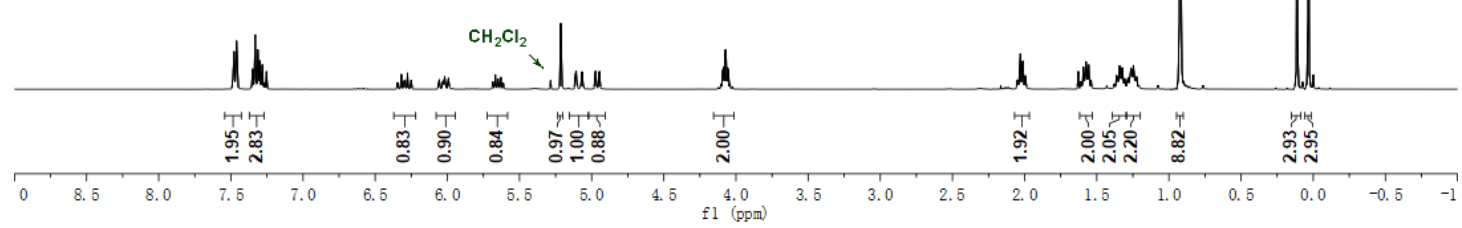

商<smiles>C=CC=CCOC(=O)C([Hg])C([O-])c1ccccc1</smiles>

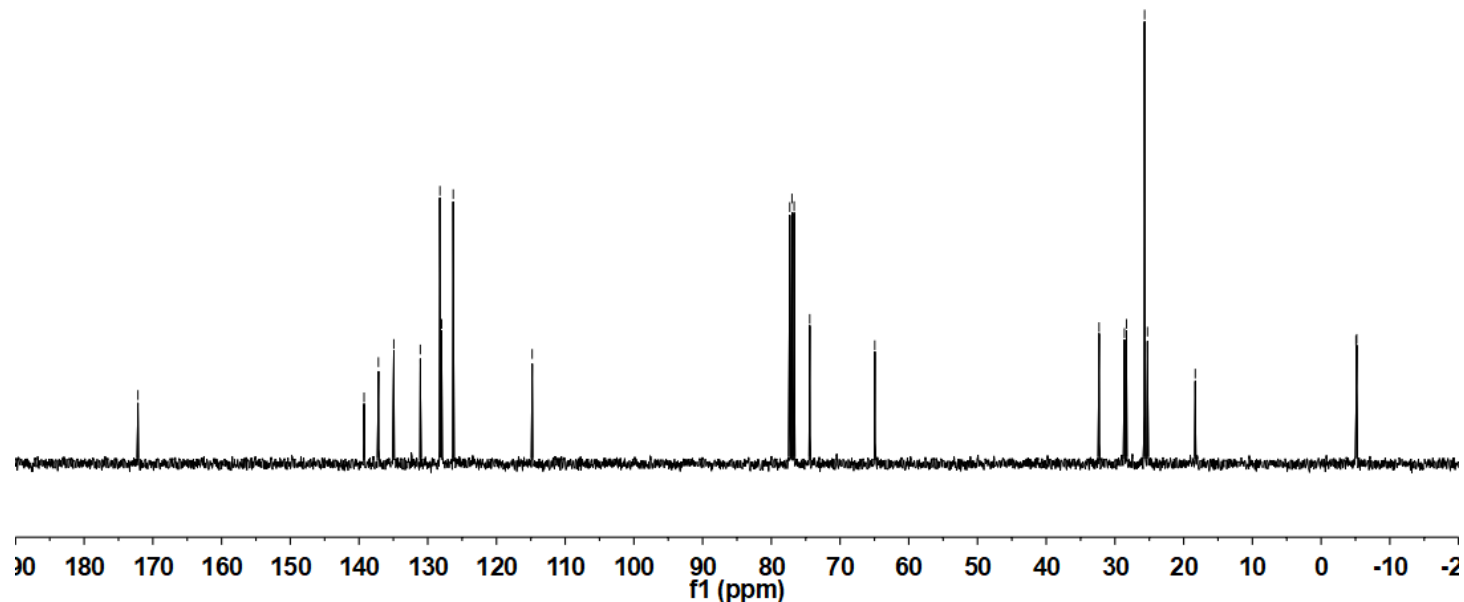


$1 \mathrm{k} ;{ }^{1} \mathrm{H}$ NMR (400MHz, $\left.\mathrm{CDCl}_{3}\right) ;{ }^{13} \mathrm{C}$ NMR $\left(100 \mathrm{MHz}, \mathrm{CDCl}_{3}\right)$

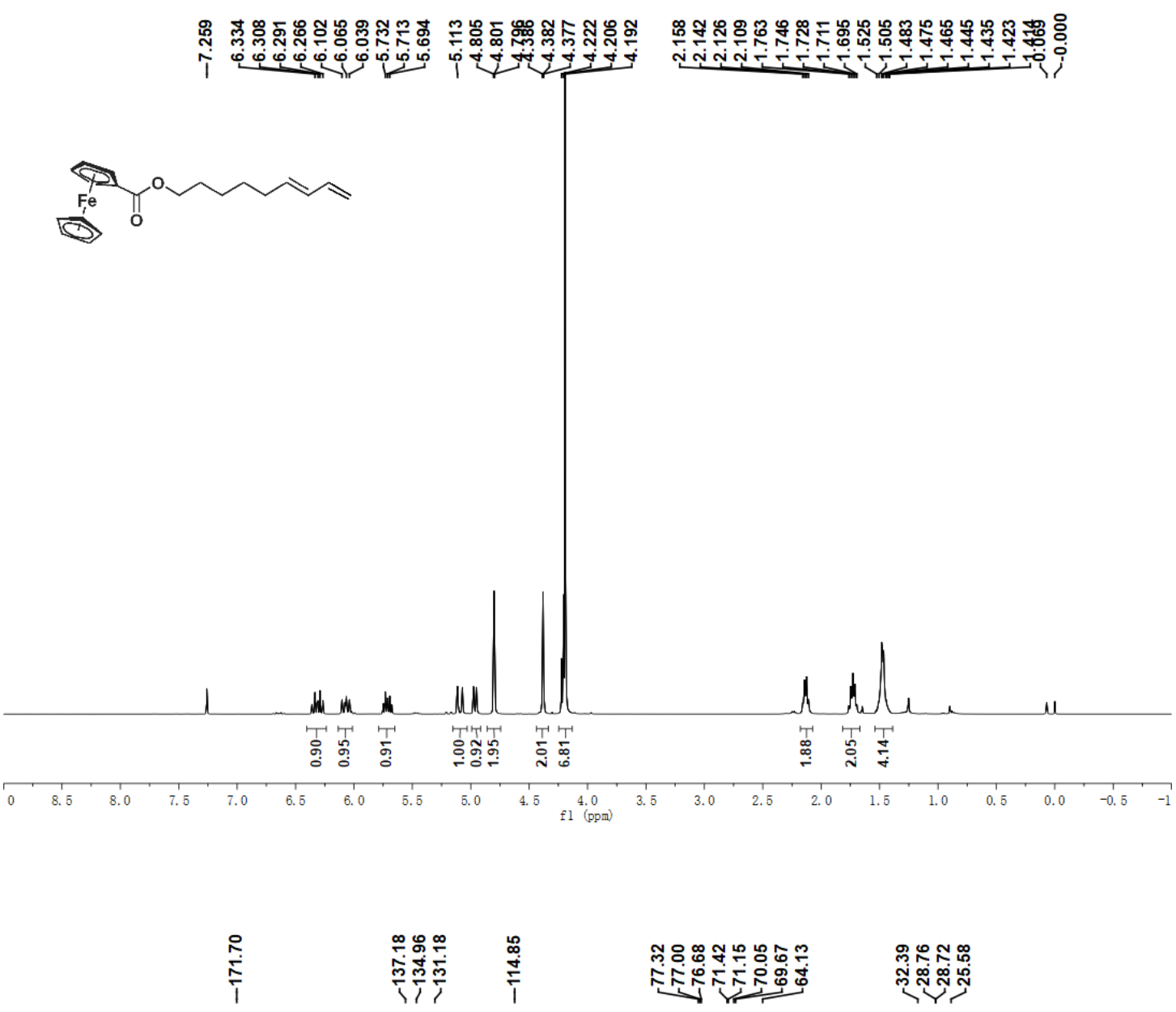

$\overbrace{\substack{\text { Fe } \\ \infty}}^{\infty} \pi_{0}$

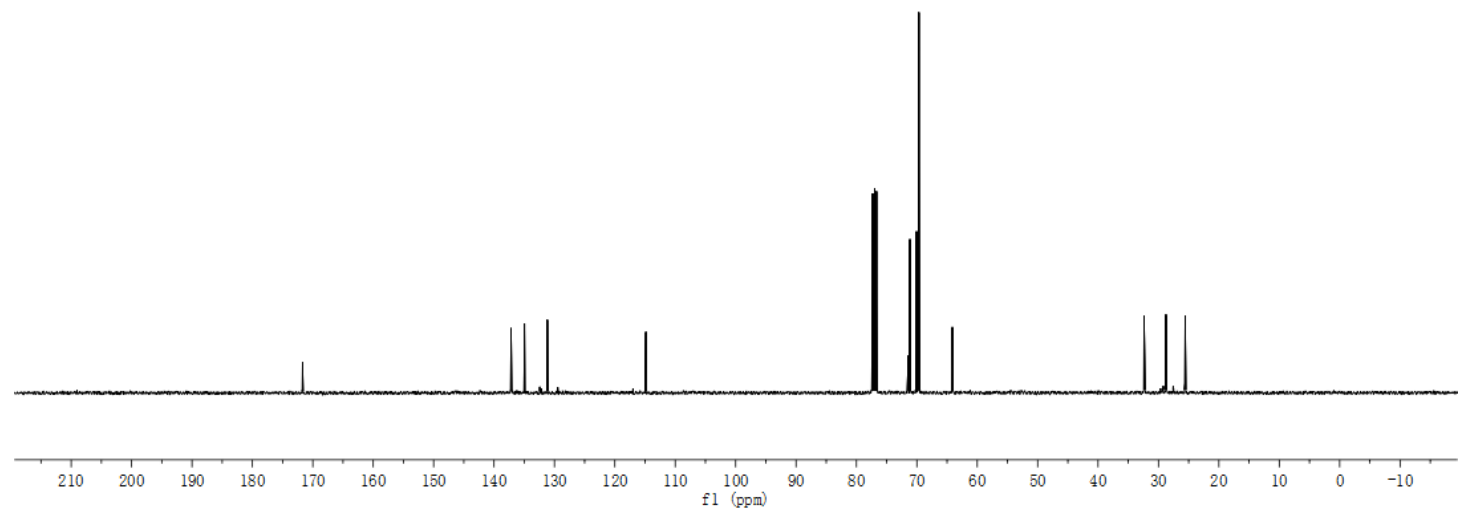


$Z$-18; ${ }^{1} \mathrm{H}$ NMR (400MHz, $\left.\mathrm{CDCl}_{3}\right) ;{ }^{13} \mathrm{C}$ NMR (100 MHz, $\left.\mathrm{CDCl}_{3}\right)$

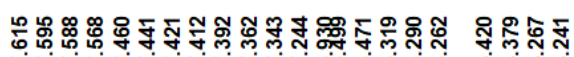

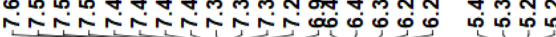

夏

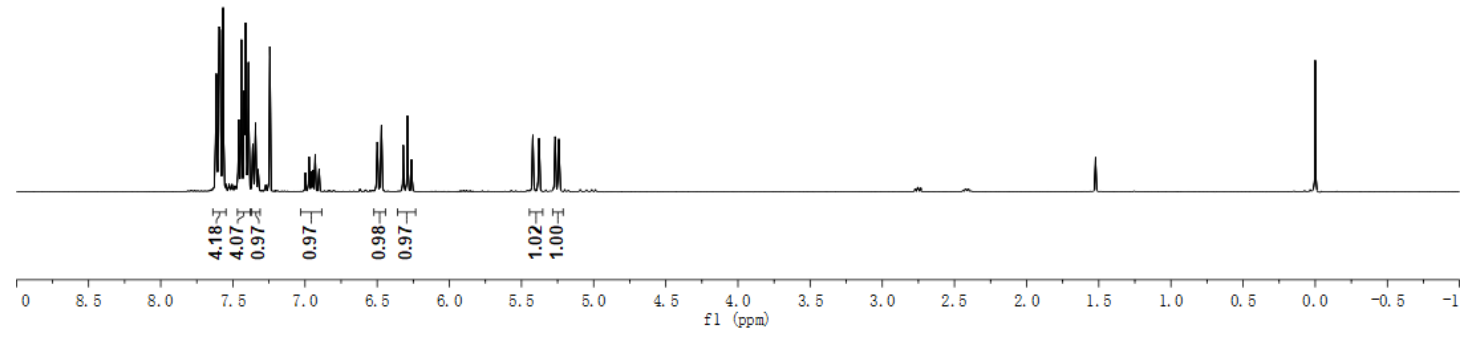

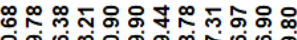

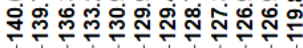

กำ:

손

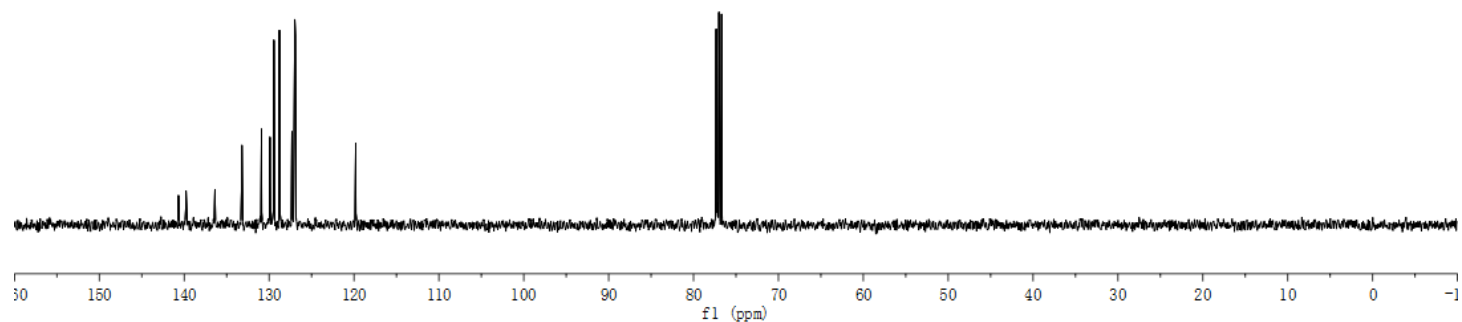


2s; ${ }^{1} \mathrm{H}$ NMR (600MHz, $\left.\mathrm{CDCl}_{3}\right) ;{ }^{13} \mathrm{C}$ NMR (150 MHz, $\left.\mathrm{CDCl}_{3}\right)$

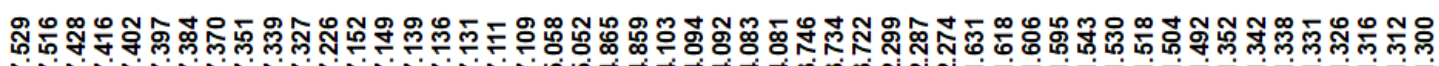

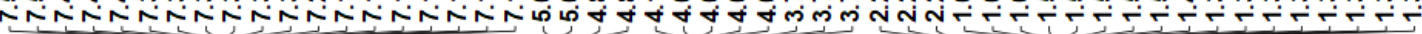<smiles></smiles>

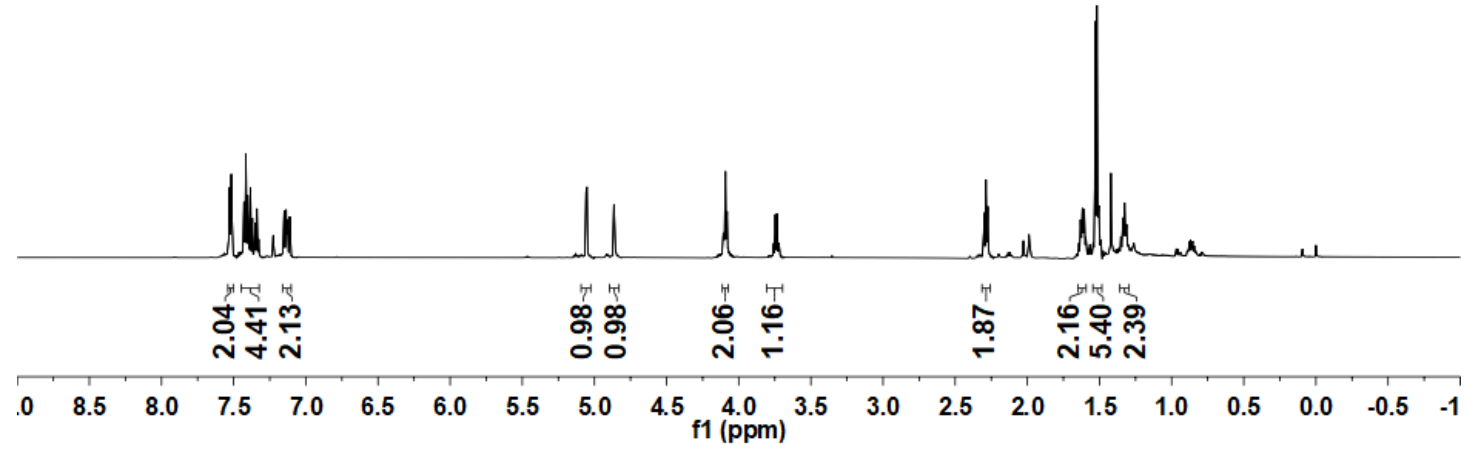

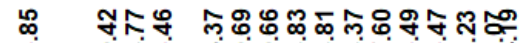

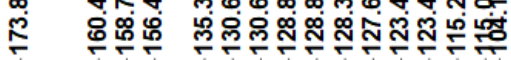

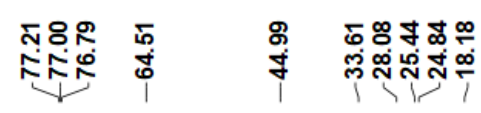<smiles>C=C(CCCCCOC(=O)C(C)c1ccc(-c2ccccc2)c(F)c1)OC</smiles>

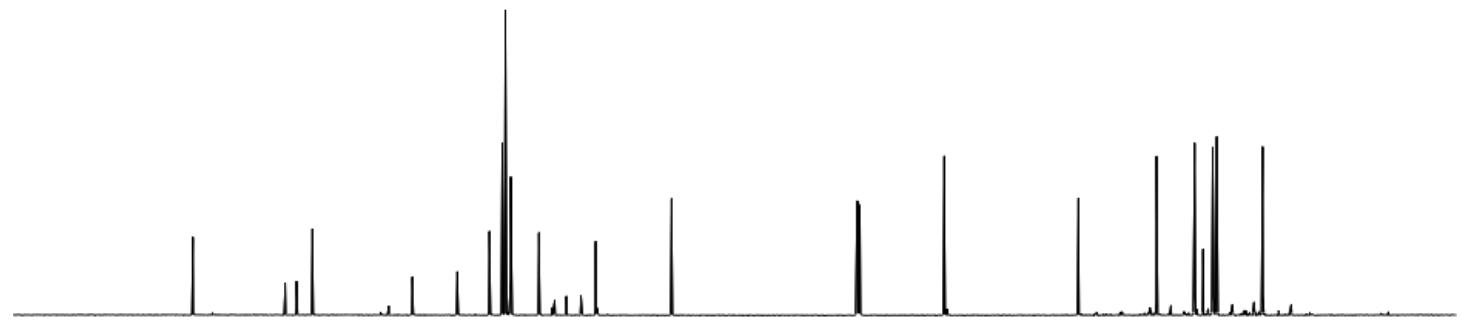

$\begin{array}{lllllllllllllllllllll}30 & 190 & 180 & 170 & 160 & 150 & 140 & 130 & 120 & 110 & \underset{f}{100} \underset{(\mathrm{ppm})}{90} & 80 & 70 & 60 & 50 & 40 & 30 & 20 & 10 & 0 & -1\end{array}$ 
2s; ${ }^{19}$ F NMR (564 MHz, $\left.\mathrm{CDCl}_{3}\right)$

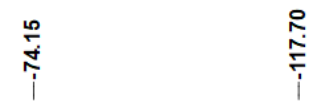

(1T)

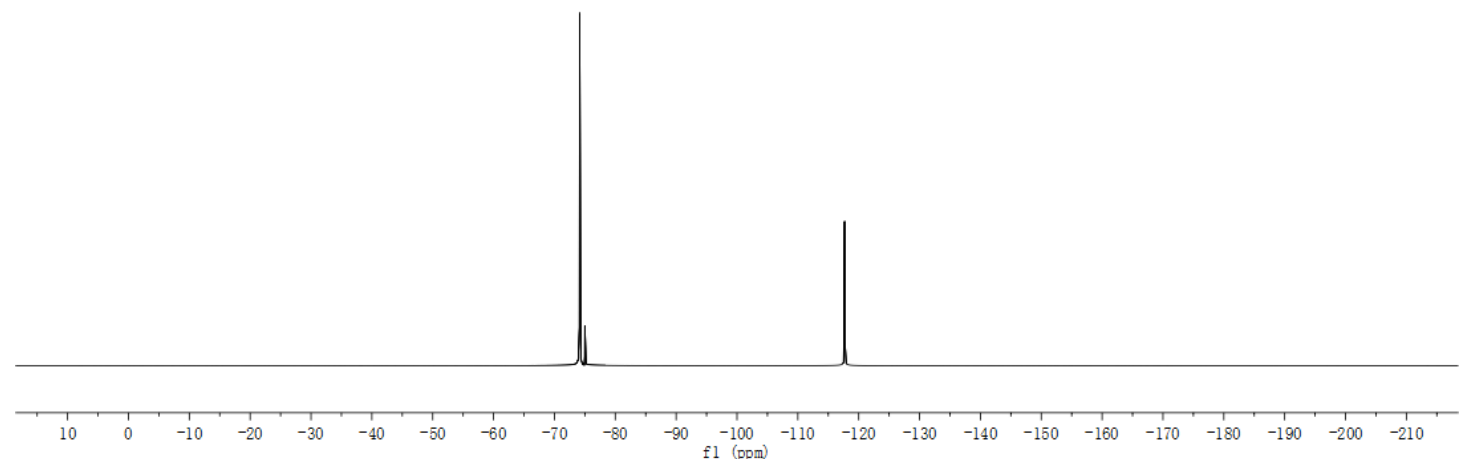


3a; ${ }^{1} \mathrm{H}$ NMR (400MHz, $\left.\mathrm{CDCl}_{3}\right) ;{ }^{13} \mathrm{C}$ NMR (100 MHz, $\left.\mathrm{CDCl}_{3}\right)$

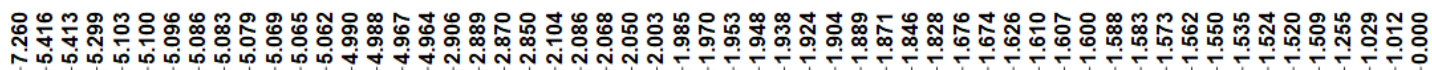

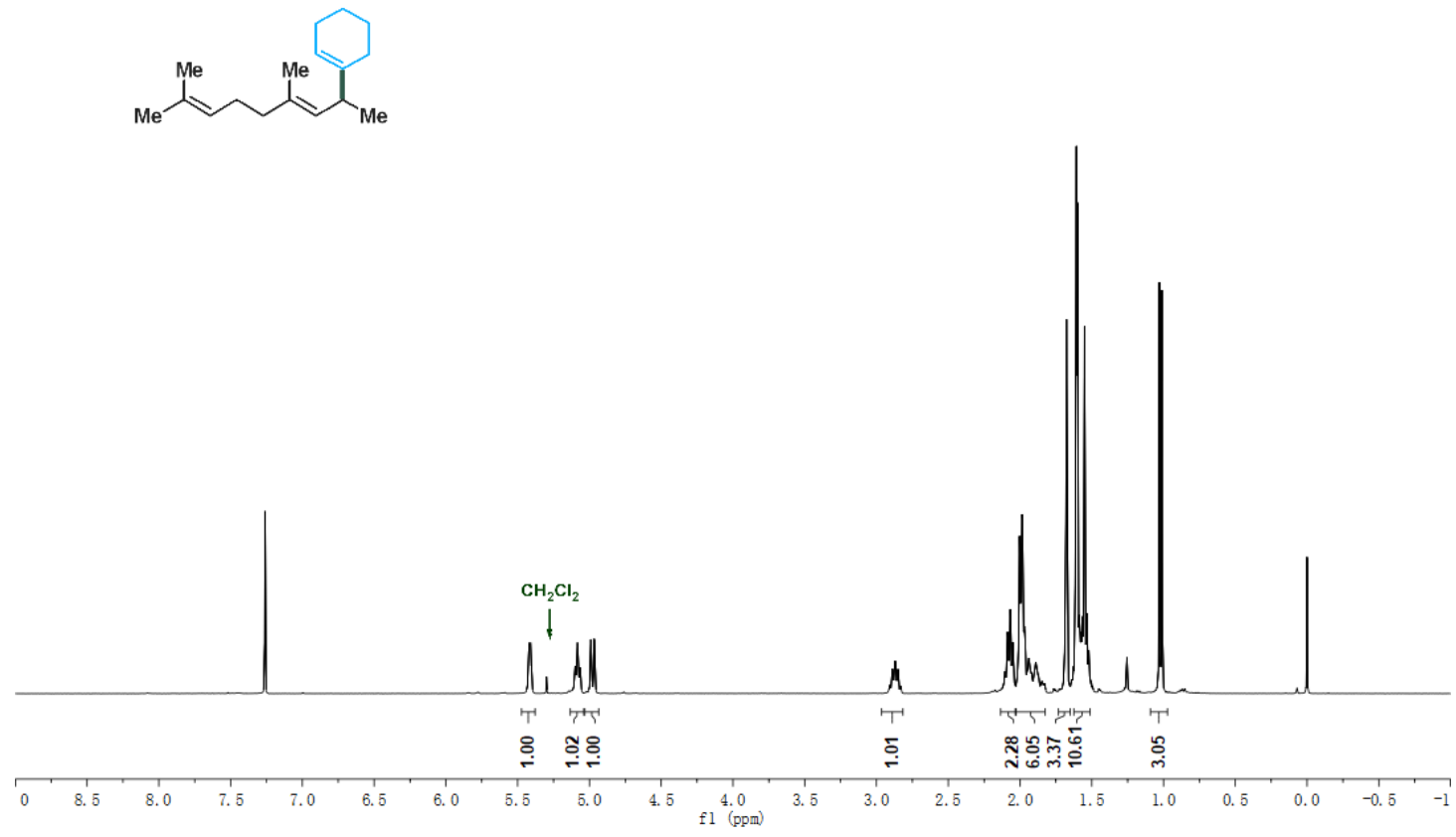

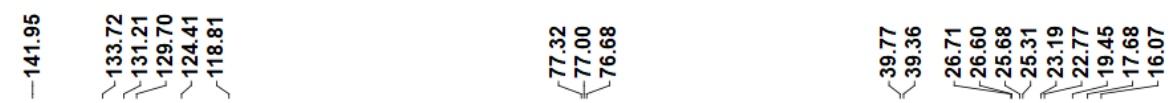
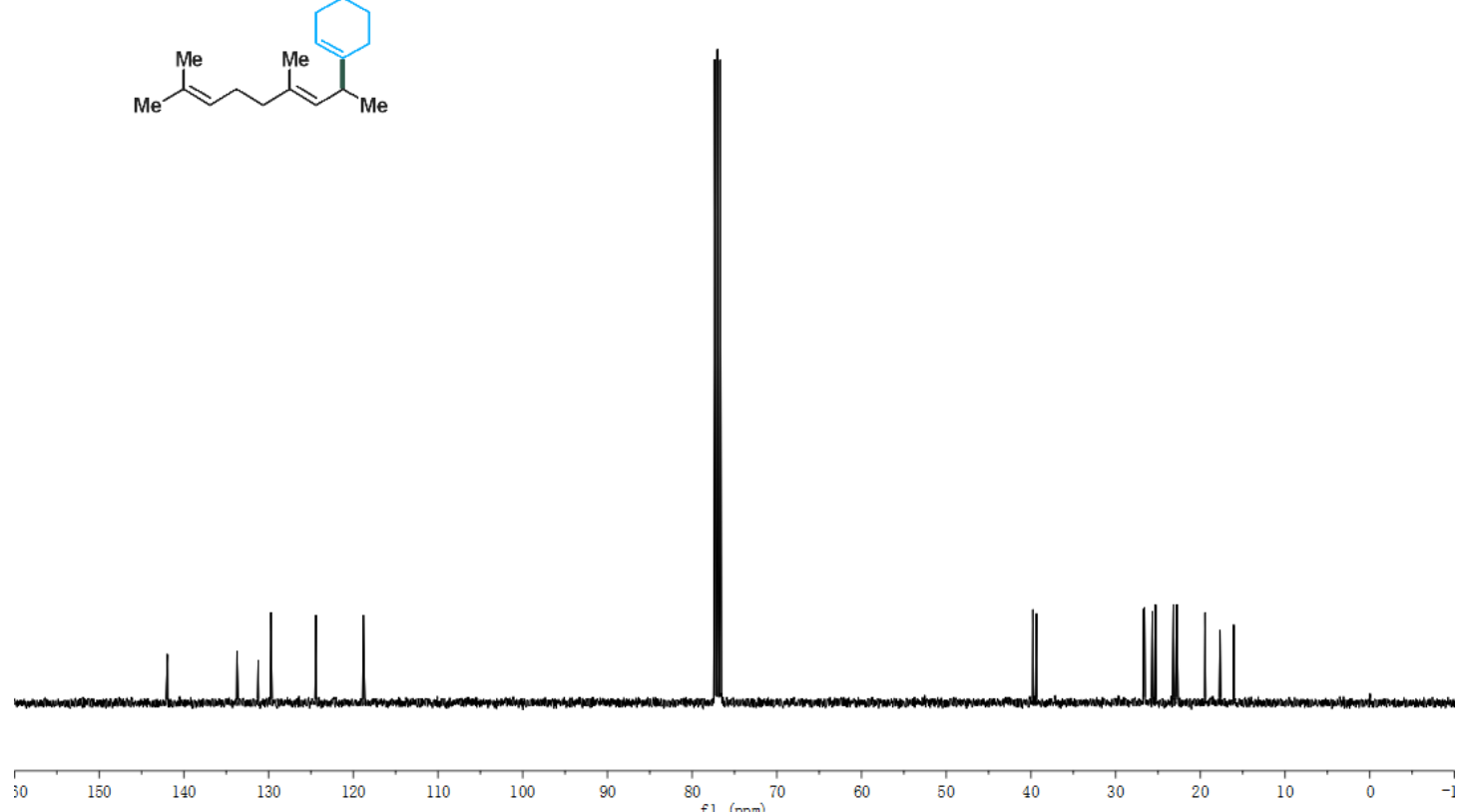
$3 \mathrm{~b} ;{ }^{1} \mathrm{H}$ NMR (400MHz, $\left.\mathrm{CDCl}_{3}\right) ;{ }^{13} \mathrm{C}$ NMR (100 MHz, $\left.\mathrm{CDCl}_{3}\right)$

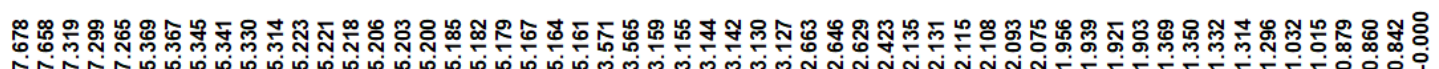

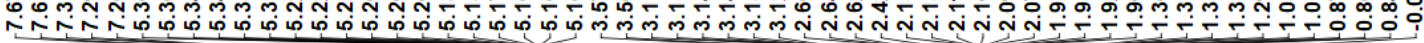<smiles>CCC/C=C/C(C)C1=CC[S+](C)(=O)CC1</smiles>

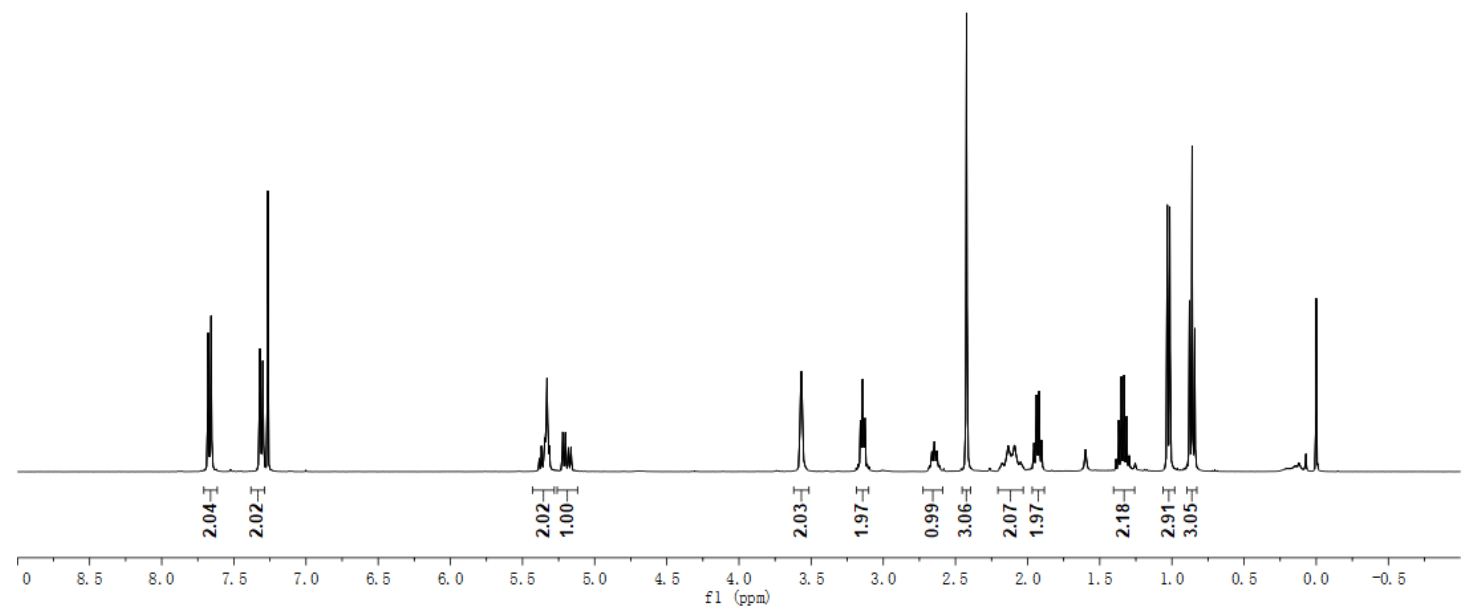<smiles>CC(C)C1CCN(C)CC1</smiles>

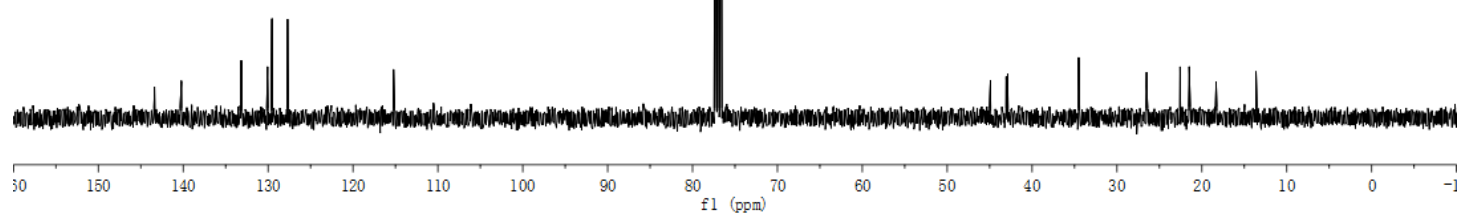


3c; ${ }^{1} \mathrm{H}$ NMR (400MHz, $\left.\mathrm{CDCl}_{3}\right) ;{ }^{13} \mathrm{C}$ NMR (100 MHz, $\left.\mathrm{CDCl}_{3}\right)$

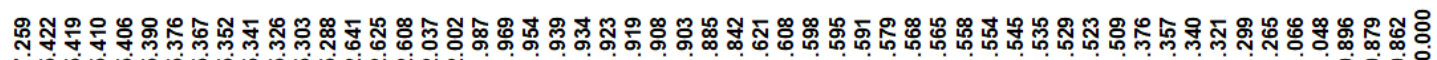

N
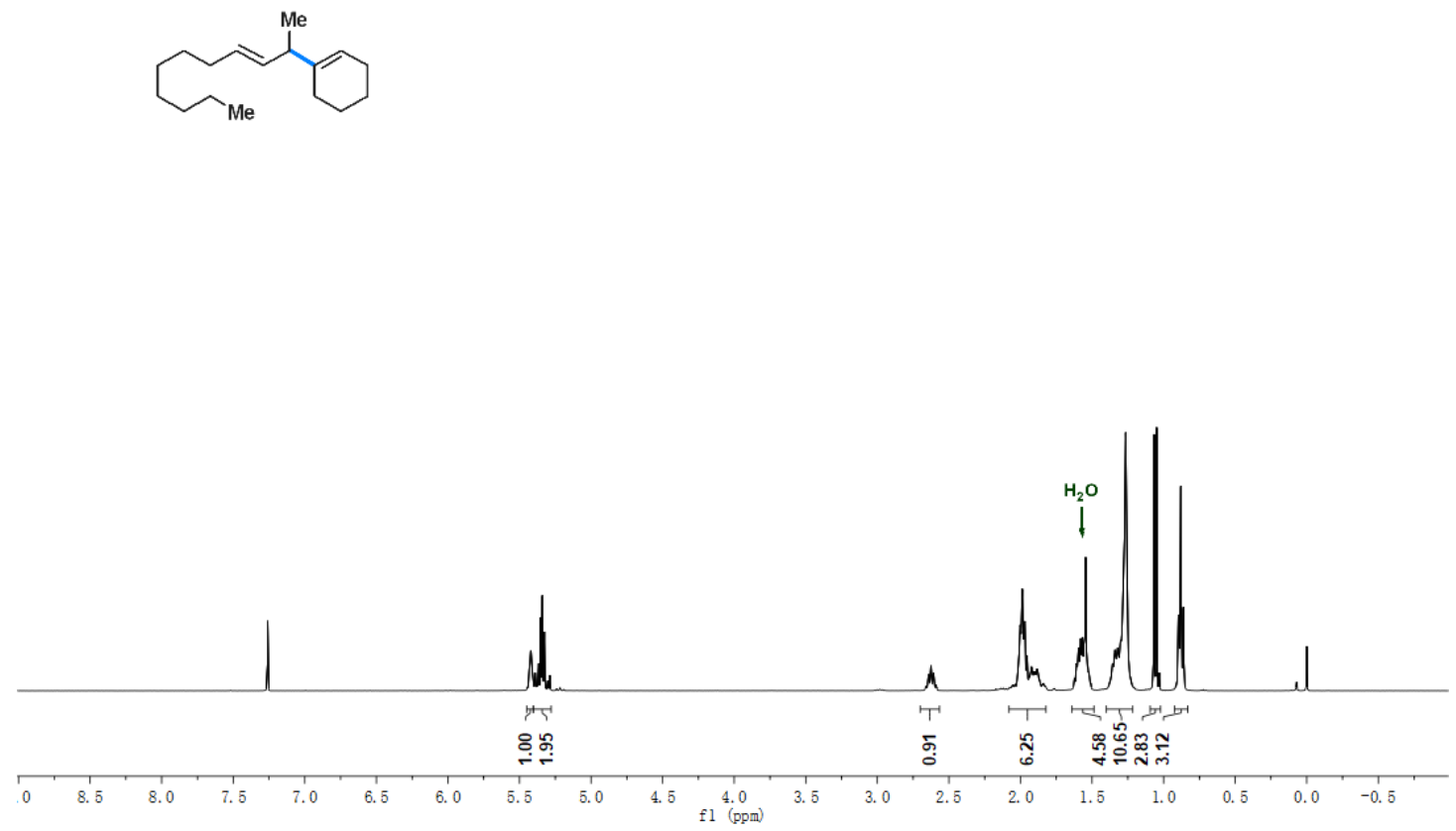

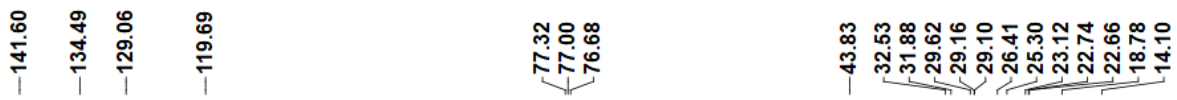
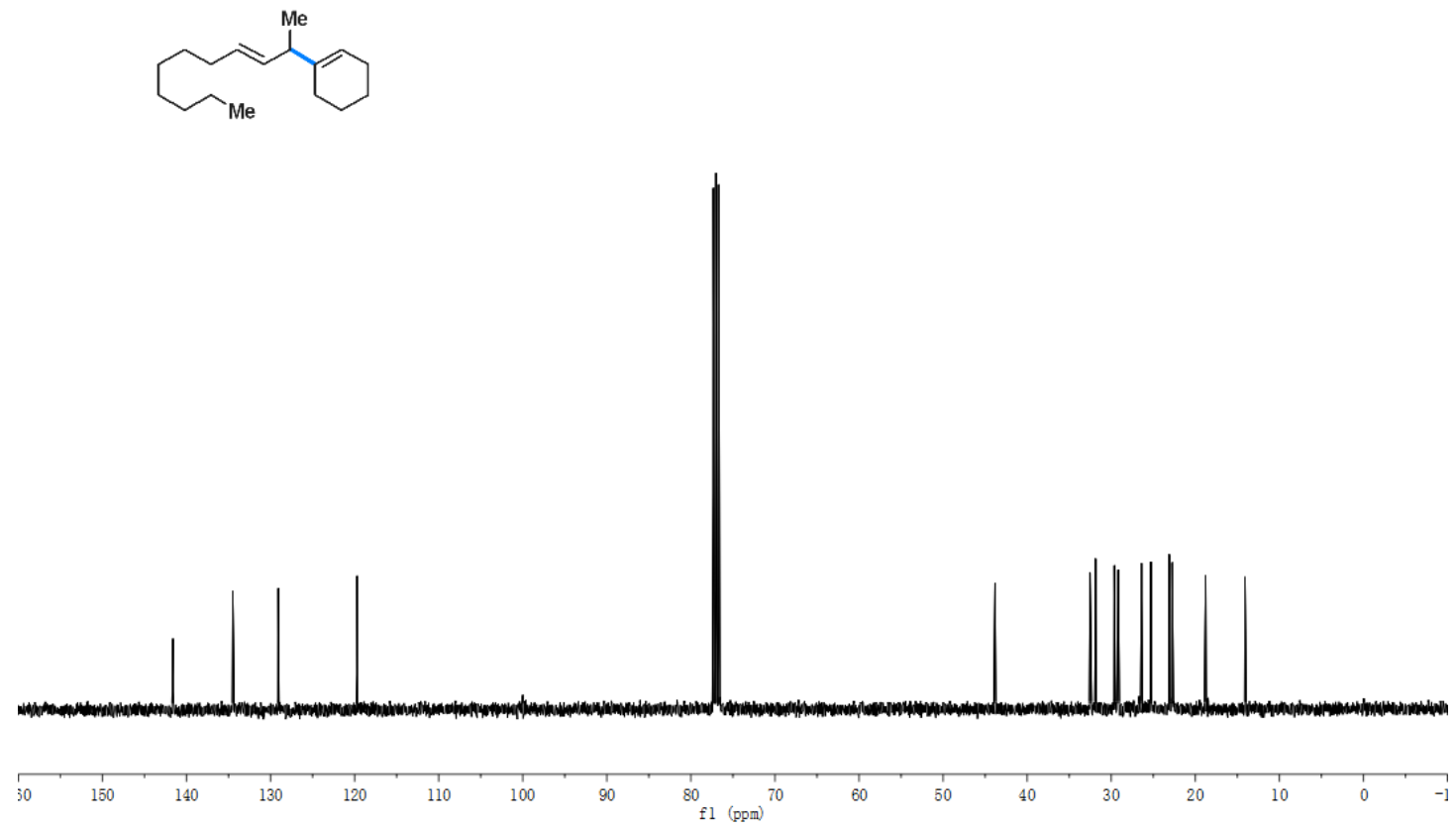
3d; ${ }^{1} \mathrm{H}$ NMR (400MHz, $\left.\mathrm{CDCl}_{3}\right) ;{ }^{13} \mathrm{C}$ NMR (100 MHz, $\left.\mathrm{CDCl}_{3}\right)$

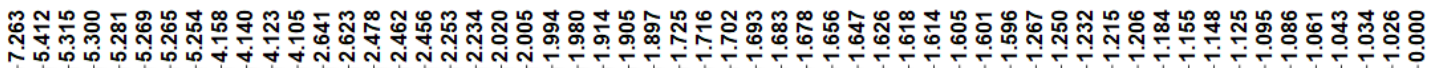<smiles>CCOC1CC=C([C@@H](C)/C=C/C2CCCCC2)CC1</smiles>

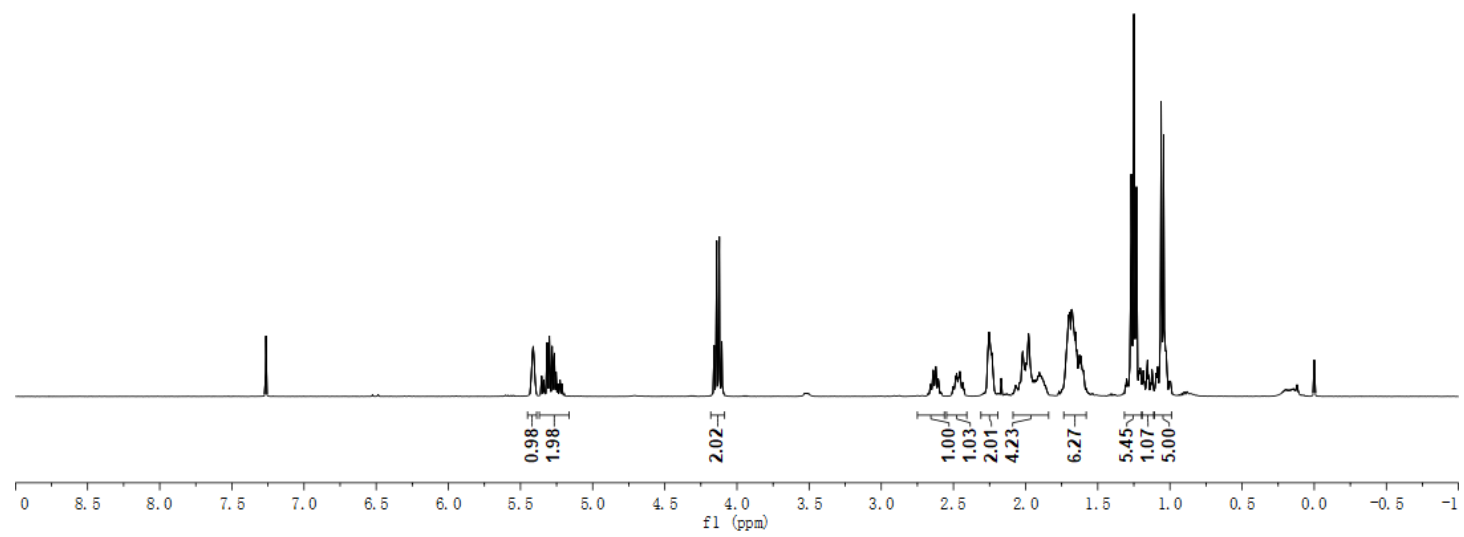

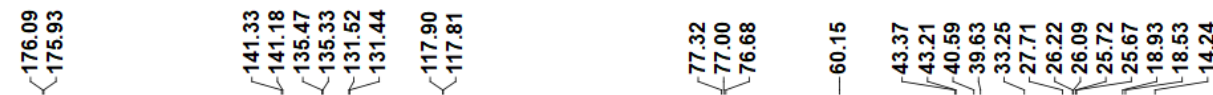<smiles>CCOC1CC=C([C@@H](C)/C=C/C2CCCCC2)CC1</smiles>

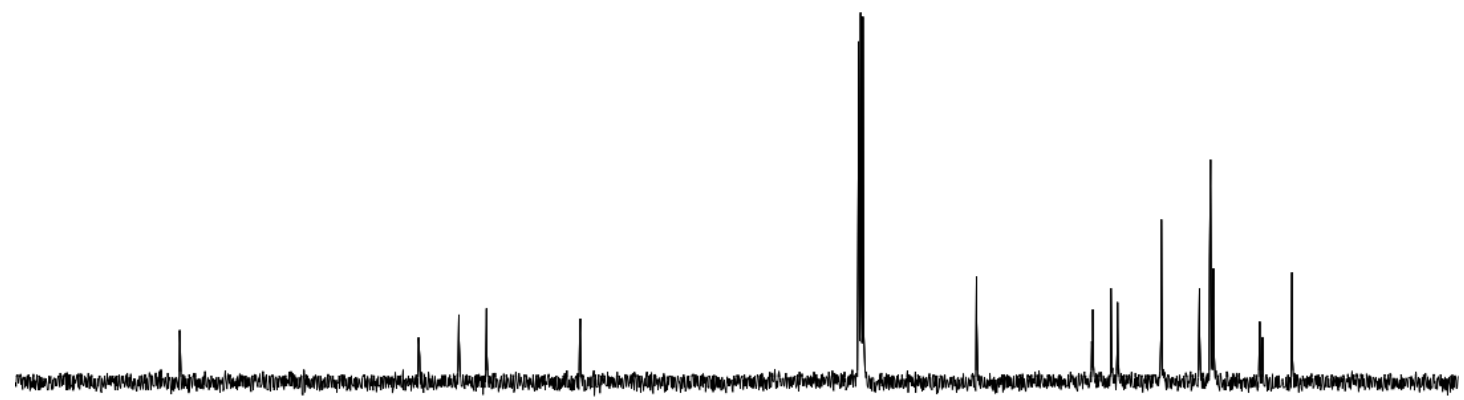

$\begin{array}{lllllllllll}190 & 180 & 170 & 160 & 150 & 140 & 130 & 120 & 110 & 100 & 90 \\ \mathrm{f} 1 & (\mathrm{ppm})\end{array}$ 
3e; ${ }^{1} \mathrm{H}$ NMR (400MHz, $\left.\mathrm{CDCl}_{3}\right) ;{ }^{13} \mathrm{C}$ NMR (100 MHz, $\left.\mathrm{CDCl}_{3}\right)$

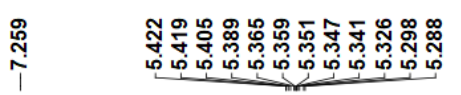

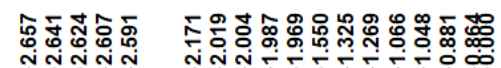

$\mathrm{H}_{17} \mathrm{C}_{8}$
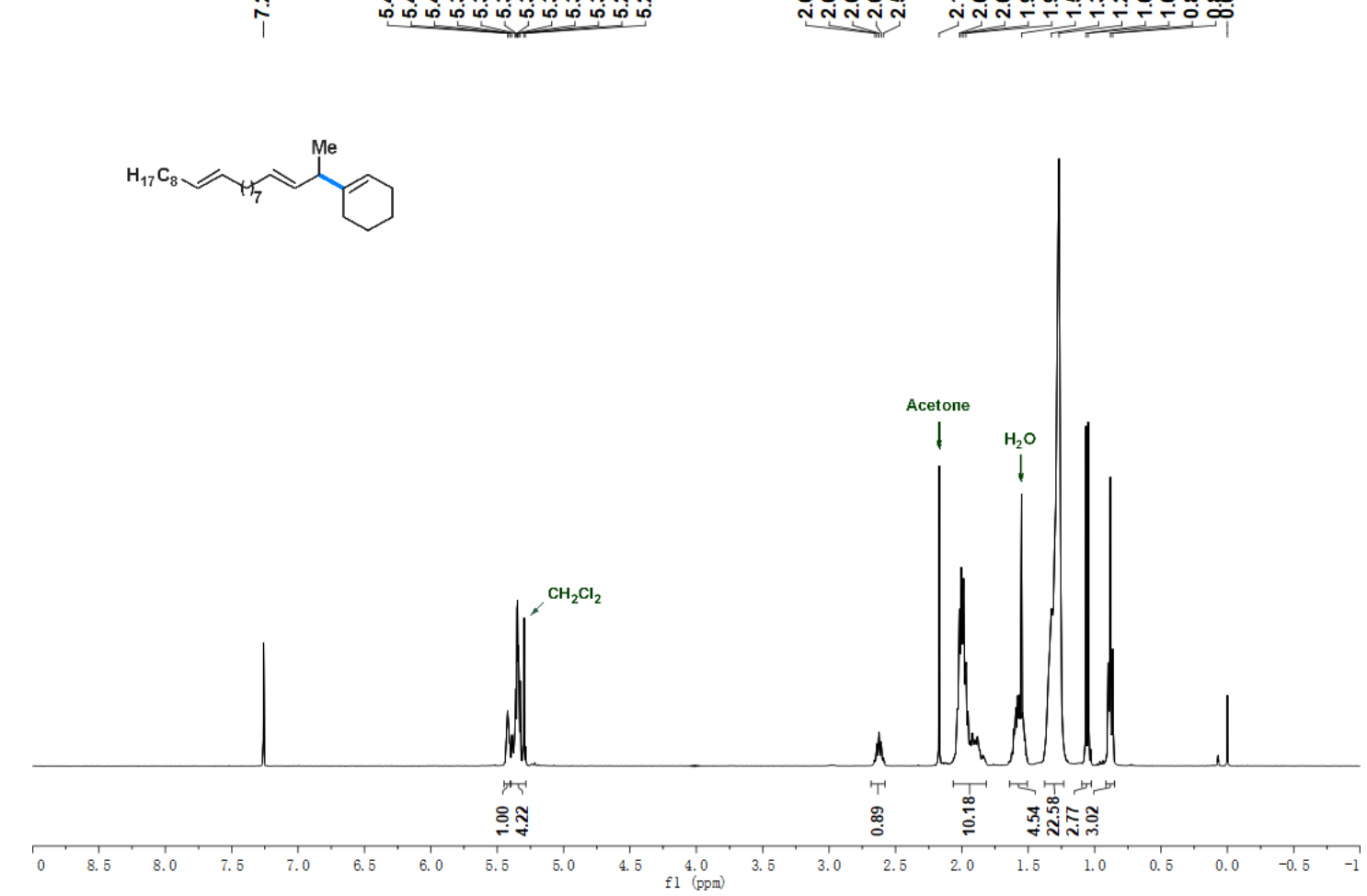

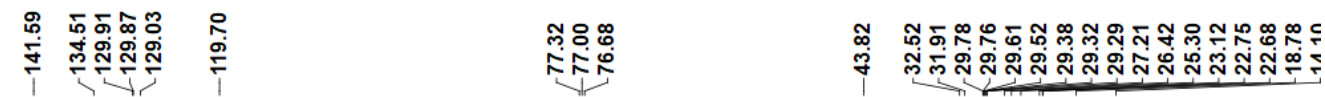

$\mathrm{H}_{17} \mathrm{C}_{8}$

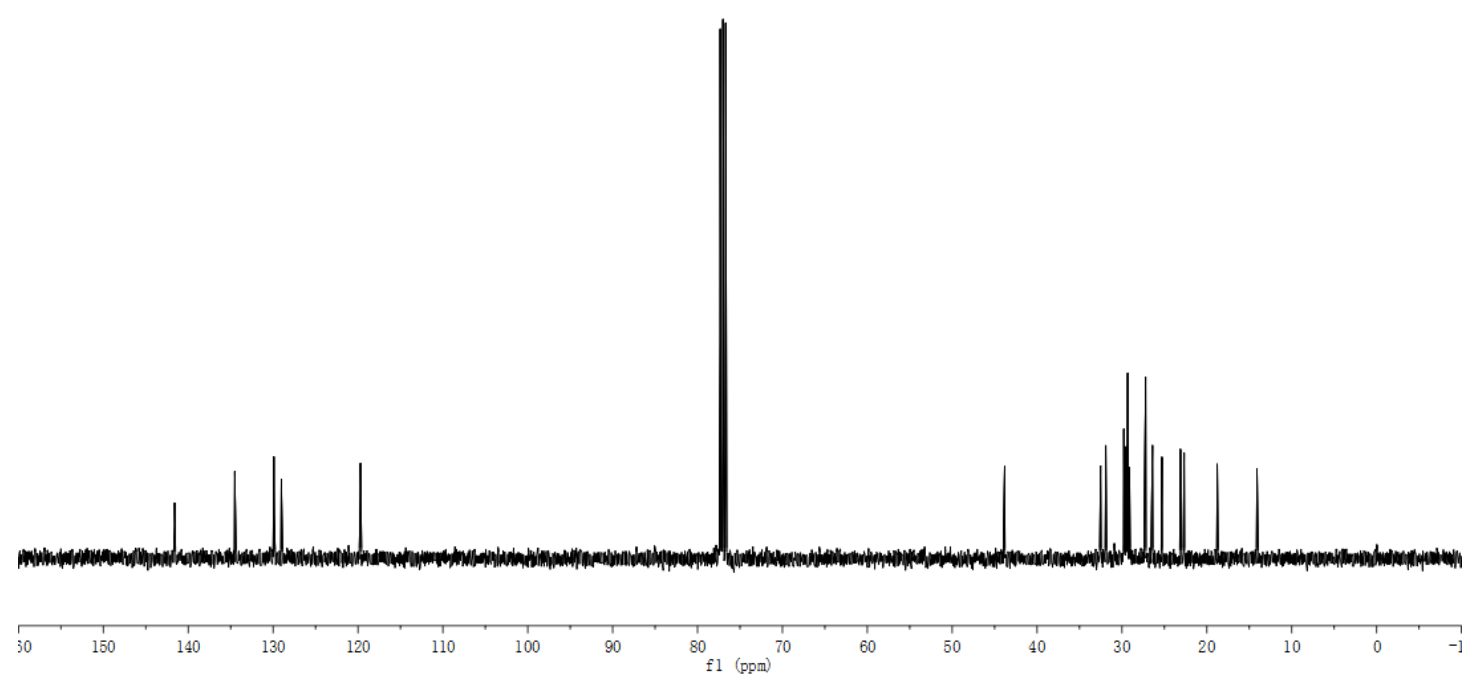




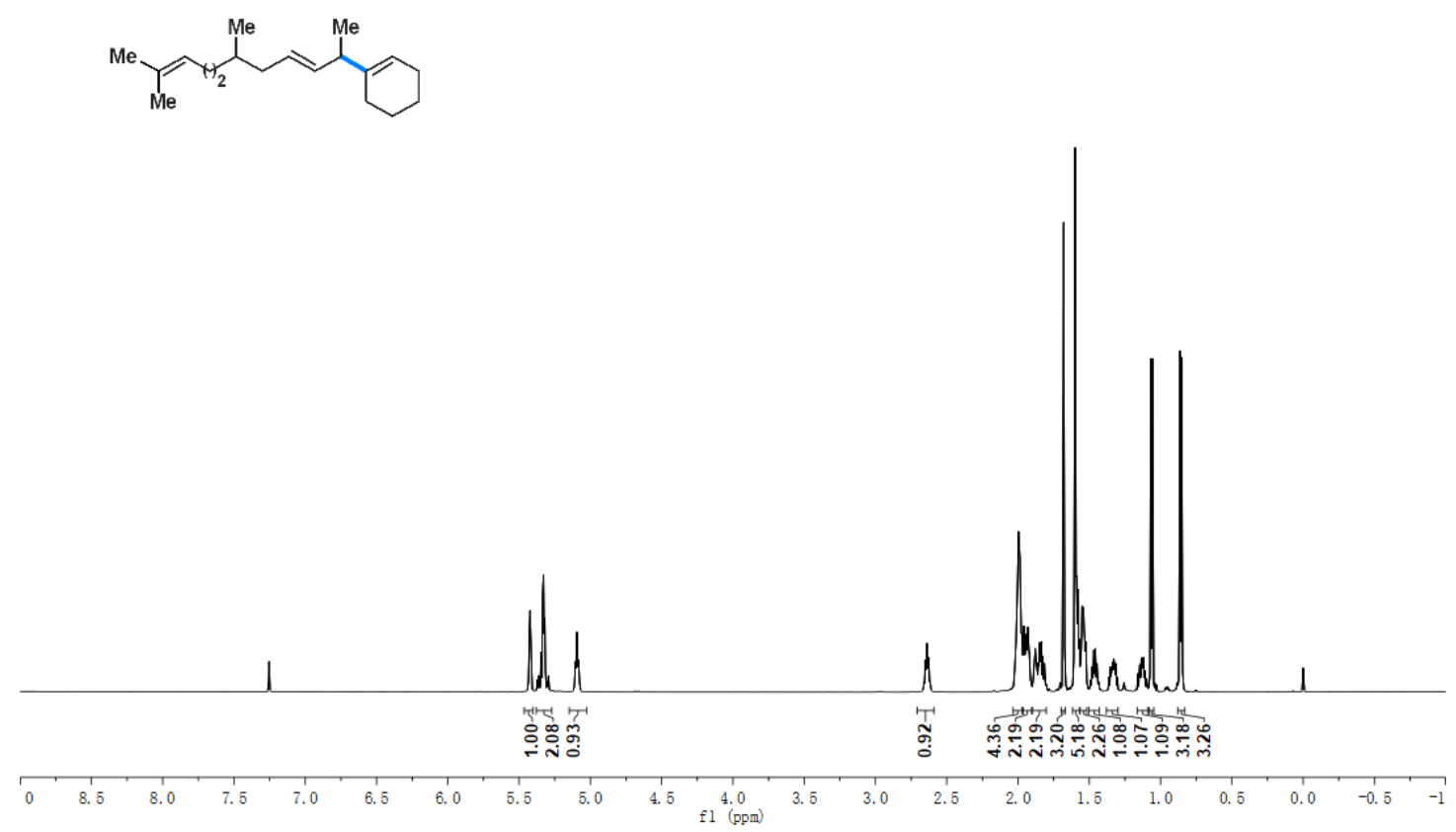

กำ

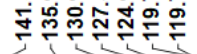

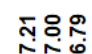

상

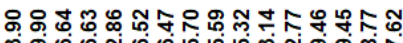

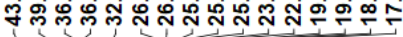<smiles>CC(C)=CCC(C)CCCC(C)C(C)C1CCCCC1</smiles>

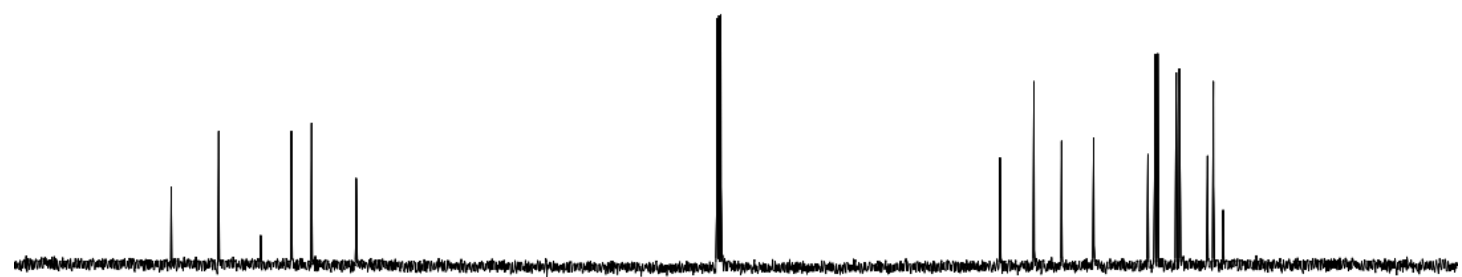

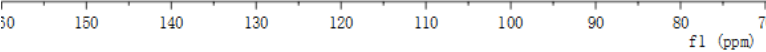


3g; ${ }^{1} \mathrm{H}$ NMR (400MHz, $\left.\mathrm{CDCl}_{3}\right) ;{ }^{13} \mathrm{C}$ NMR (100 MHz, $\left.\mathrm{CDCl}_{3}\right)$

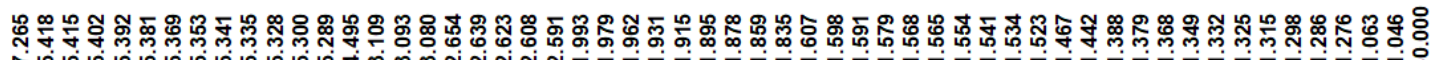

人i

(1)

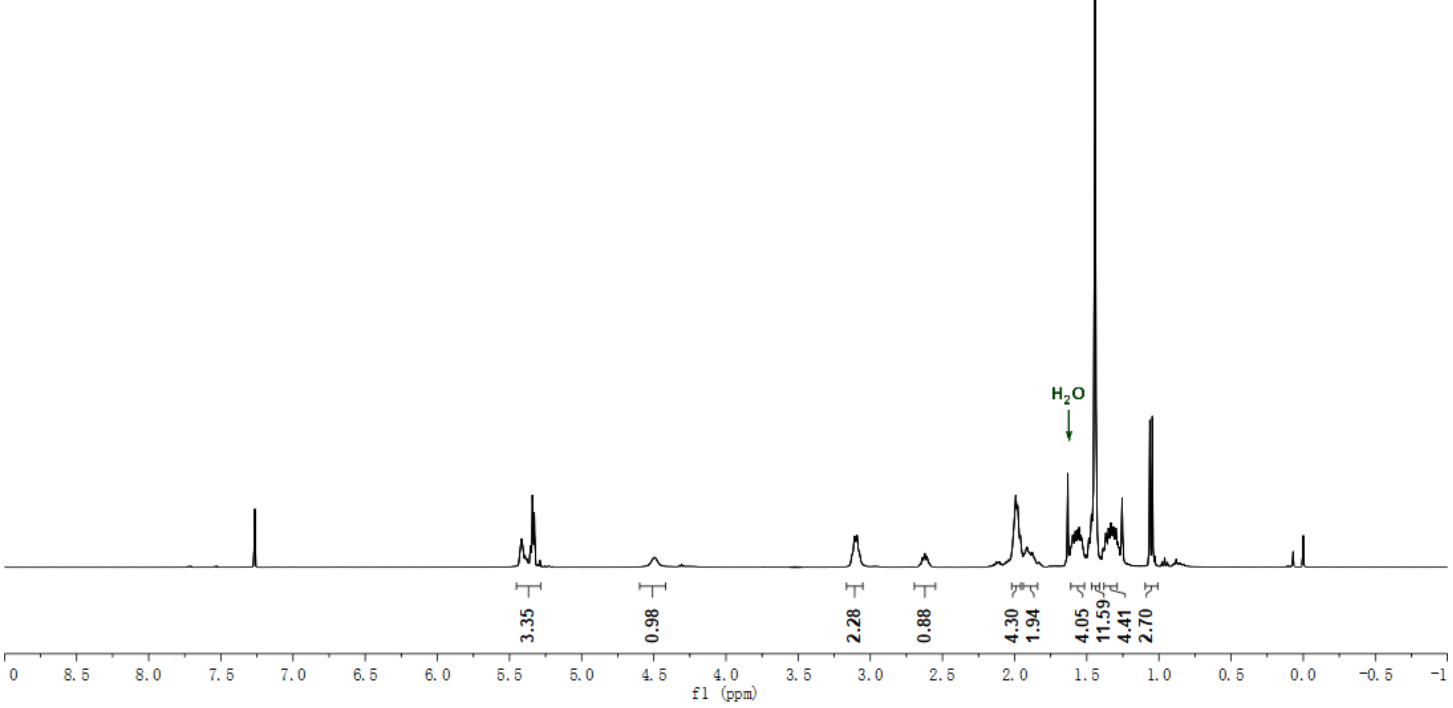

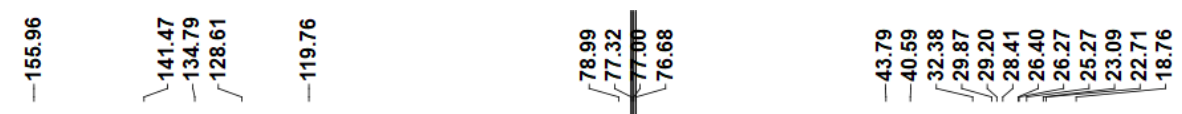

(Noc)

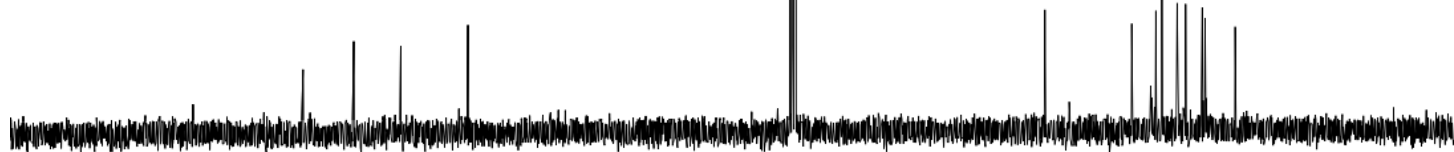

$\begin{array}{lllllllll}170 & 160 & 150 & 140 & 130 & 120 & 110 & 100 & 90 \\ \mathrm{f} 1(\mathrm{ppm}) & 80\end{array}$ 
3h; ${ }^{1} \mathrm{H}$ NMR (400MHz, $\left.\mathrm{CDCl}_{3}\right) ;{ }^{13} \mathrm{C}$ NMR (100 MHz, $\left.\mathrm{CDCl}_{3}\right)$

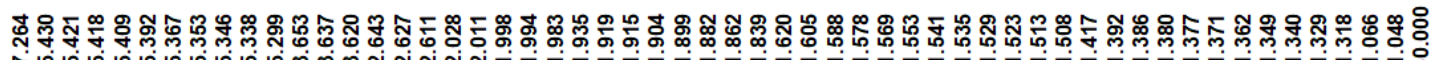

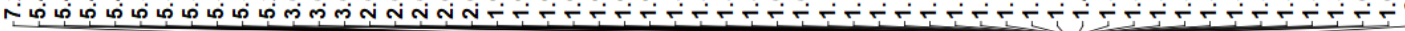<smiles>CC(/C=C/CO)C1=CCCCC1</smiles>

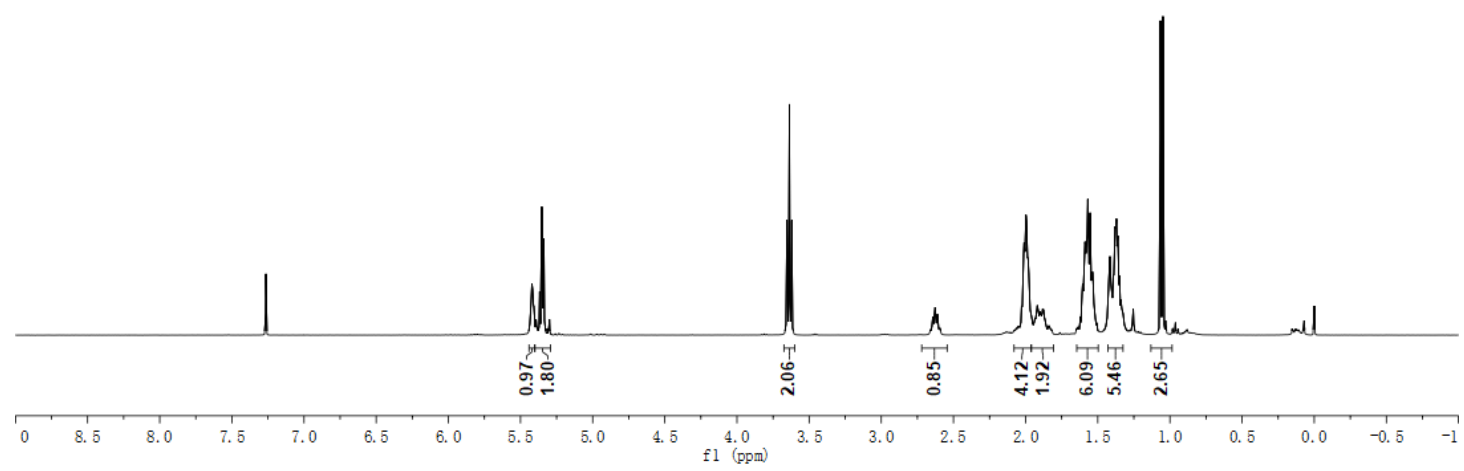

卢<smiles>CC(/C=C/CO)C1=CCCCC1</smiles>

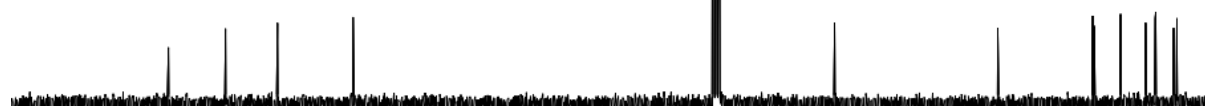

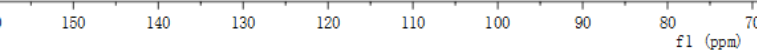


3i; ${ }^{1} \mathrm{H}$ NMR (400MHz, $\left.\mathrm{CDCl}_{3}\right) ;{ }^{13} \mathrm{C}$ NMR (100 MHz, $\left.\mathrm{CDCl}_{3}\right)$

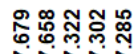

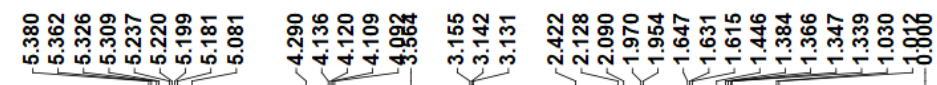

$\prod_{0}^{\mathrm{NHBoc}}$

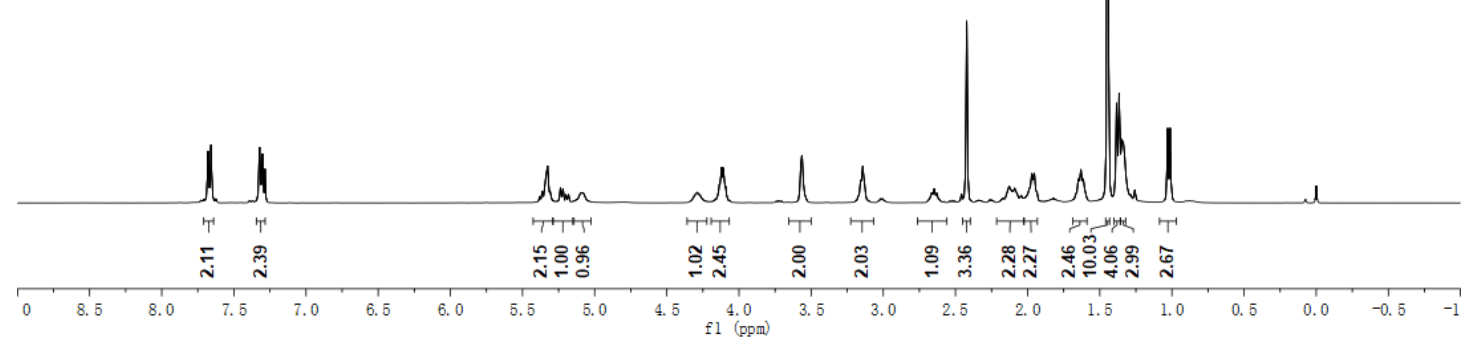

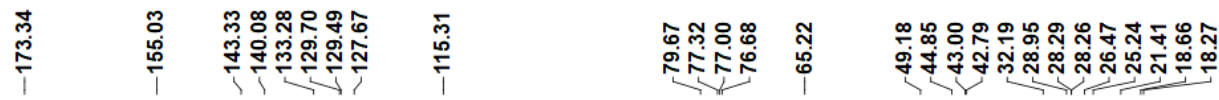

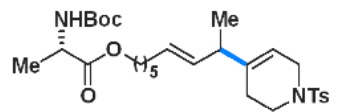

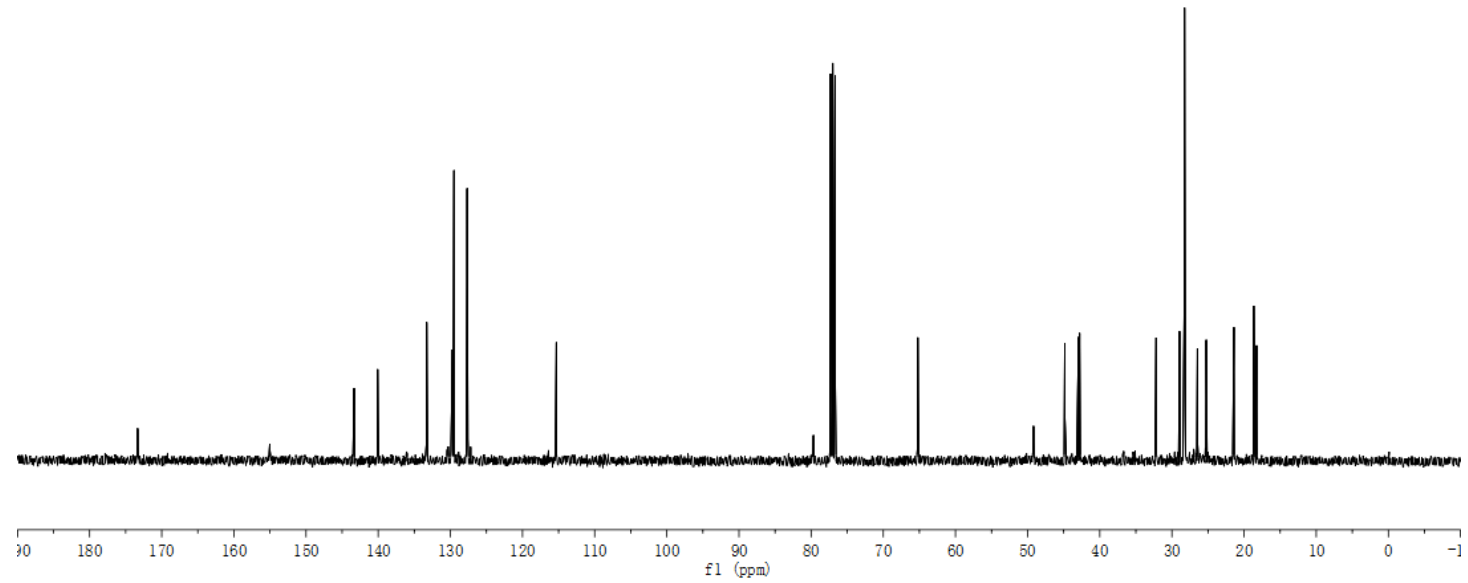


3j; ${ }^{1} \mathrm{H}$ NMR (400MHz, $\left.\mathrm{CDCl}_{3}\right) ;{ }^{13} \mathrm{C}$ NMR (100 MHz, $\left.\mathrm{CDCl}_{3}\right)$

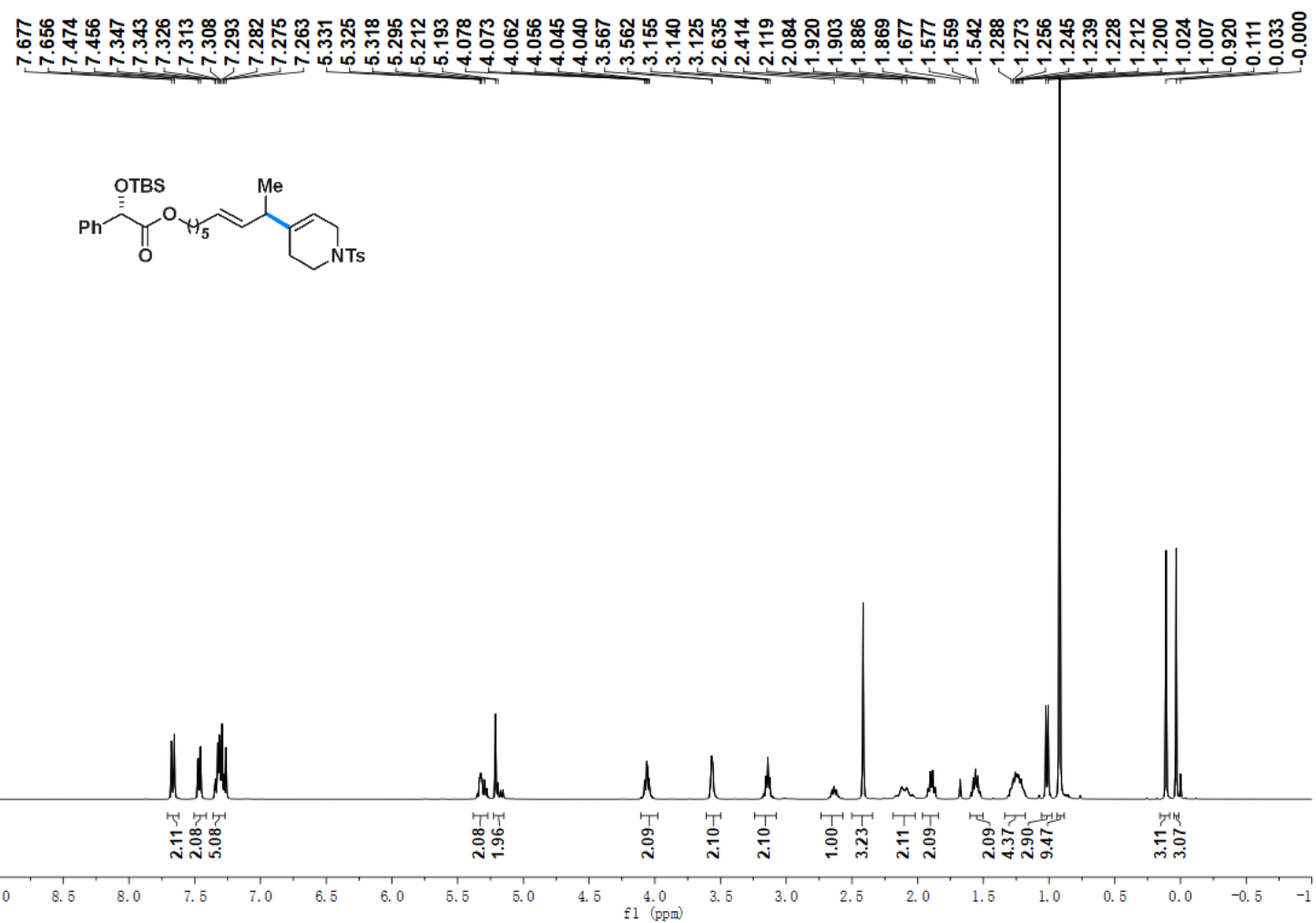

萡

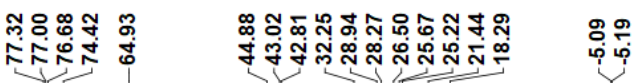<smiles>C[C@H](/C=C/COC(=O)[C@@H](O)c1ccccc1)C1=CC[NH+](C)CC1</smiles>

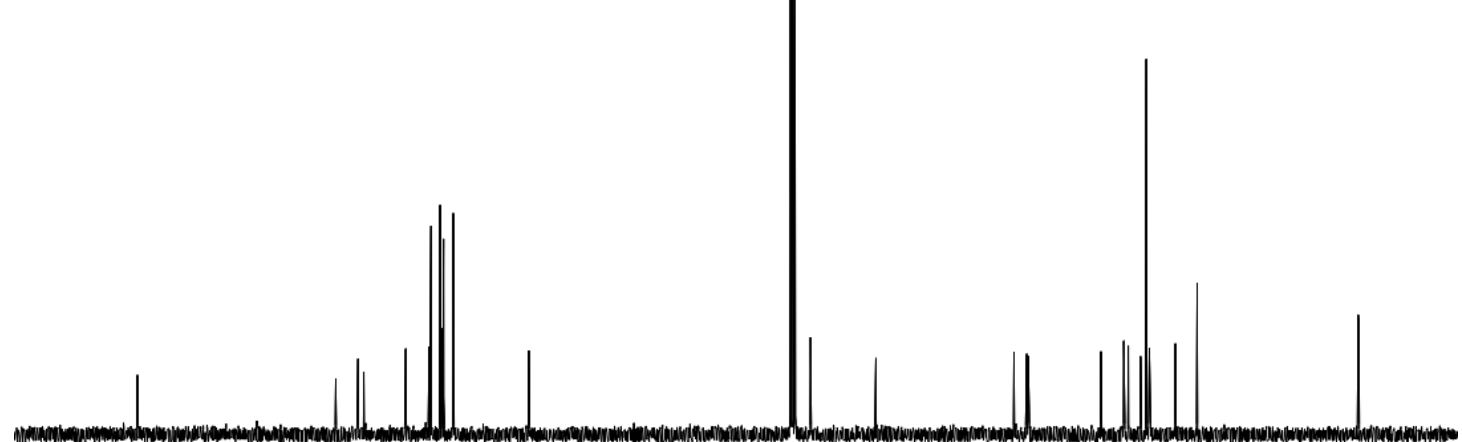

$\begin{array}{lllllllllll}180 & 170 & 160 & 150 & 140 & 130 & 120 & 110 & 100 & 90 & 90 \\ \mathrm{f} 1(\mathrm{pon}) & 80\end{array}$ 
$3 \mathrm{k} ;{ }^{1} \mathrm{H}$ NMR (400MHz, $\left.\mathrm{CDCl}_{3}\right) ;{ }^{13} \mathrm{C}$ NMR (100 MHz, $\left.\mathrm{CDCl}_{3}\right)$

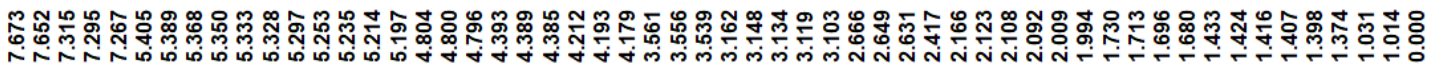

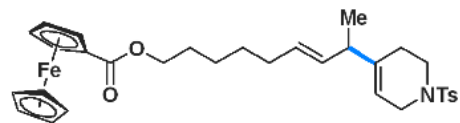

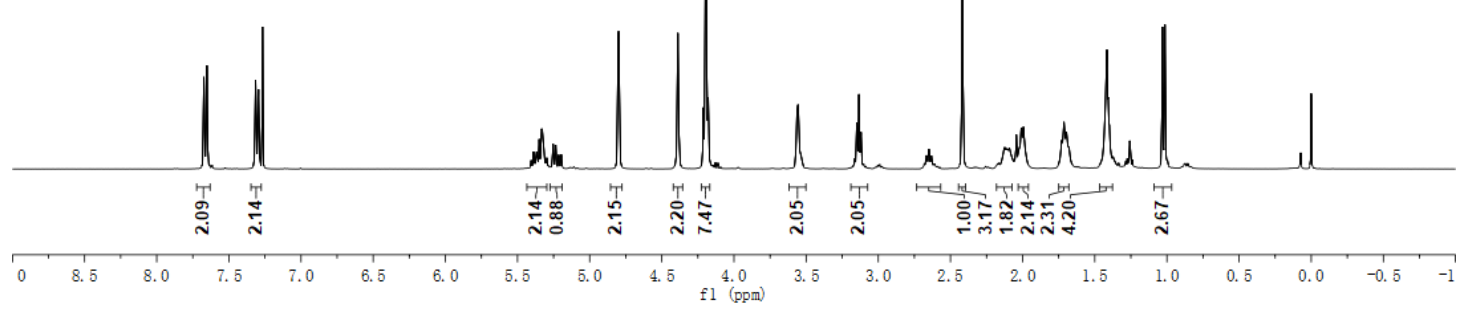

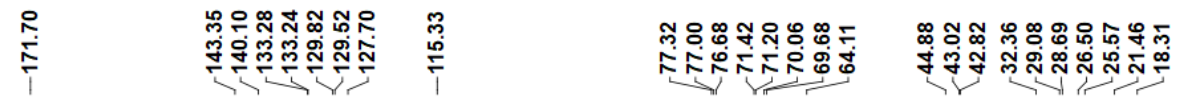

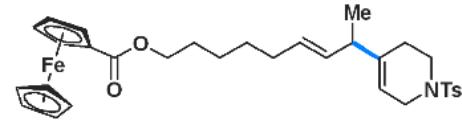

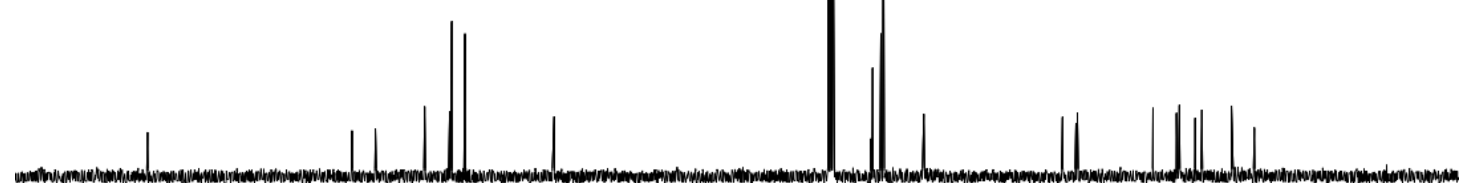

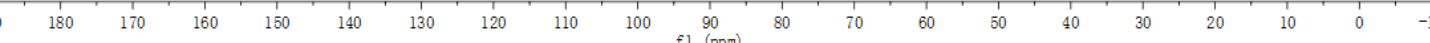


31; ${ }^{1} \mathrm{H}$ NMR (400MHz, CDCl3); ${ }^{13} \mathrm{C}$ NMR (100 MHz, CDCl3)

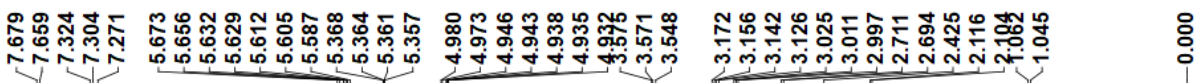<smiles>C=CCCC1=CC[NH+](C)CC1</smiles>

31

$3 \mathrm{I}-1$

$31-2$

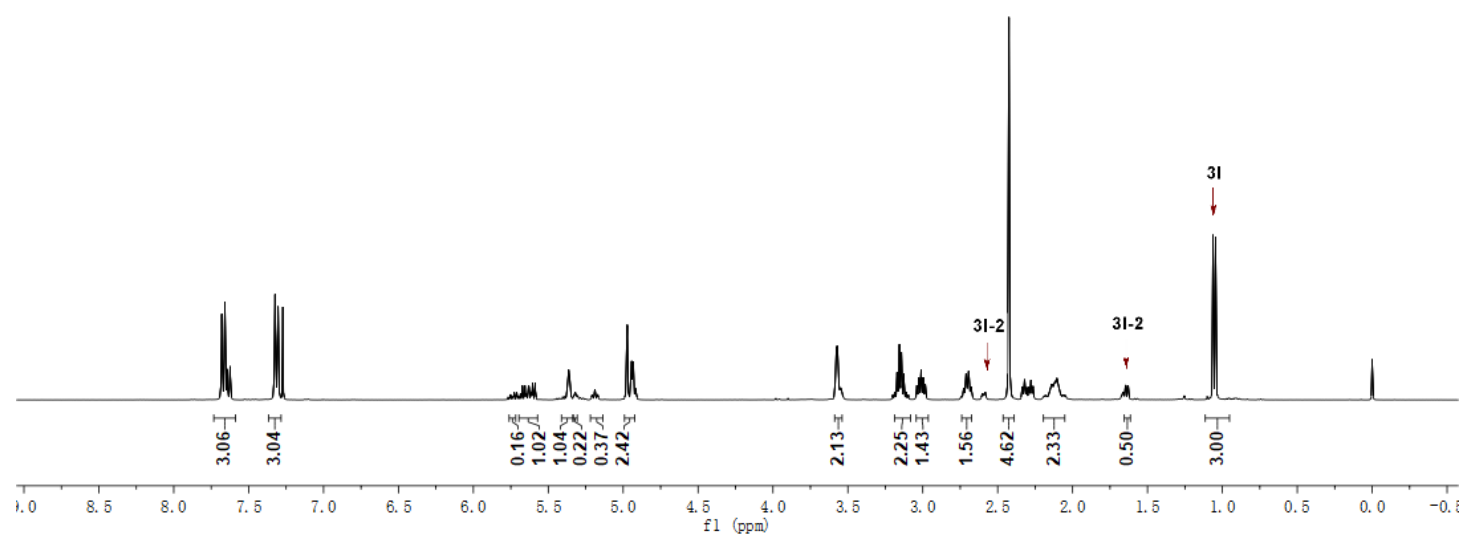

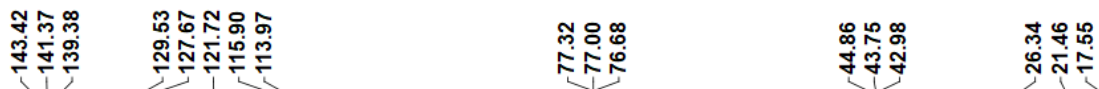

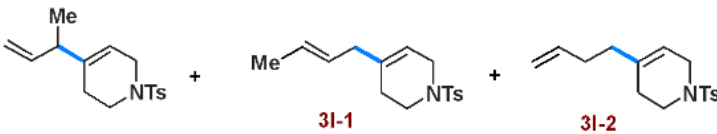

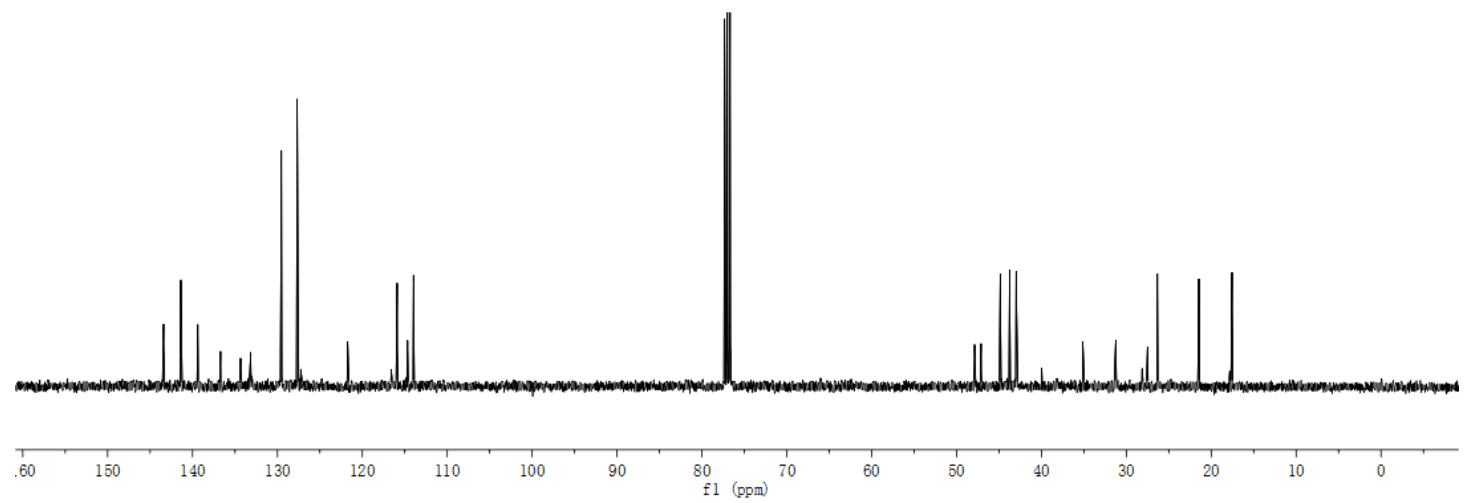


3n; ${ }^{1} \mathrm{H}$ NMR (400MHz, $\left.\mathrm{CDCl}_{3}\right) ;{ }^{13} \mathrm{C}$ NMR (100 MHz, $\left.\mathrm{CDCl}_{3}\right)$

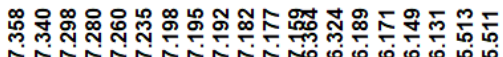

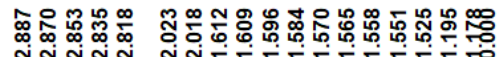<smiles>[CH](/C=C/c1ccccc1)C1=CCCCC1</smiles>

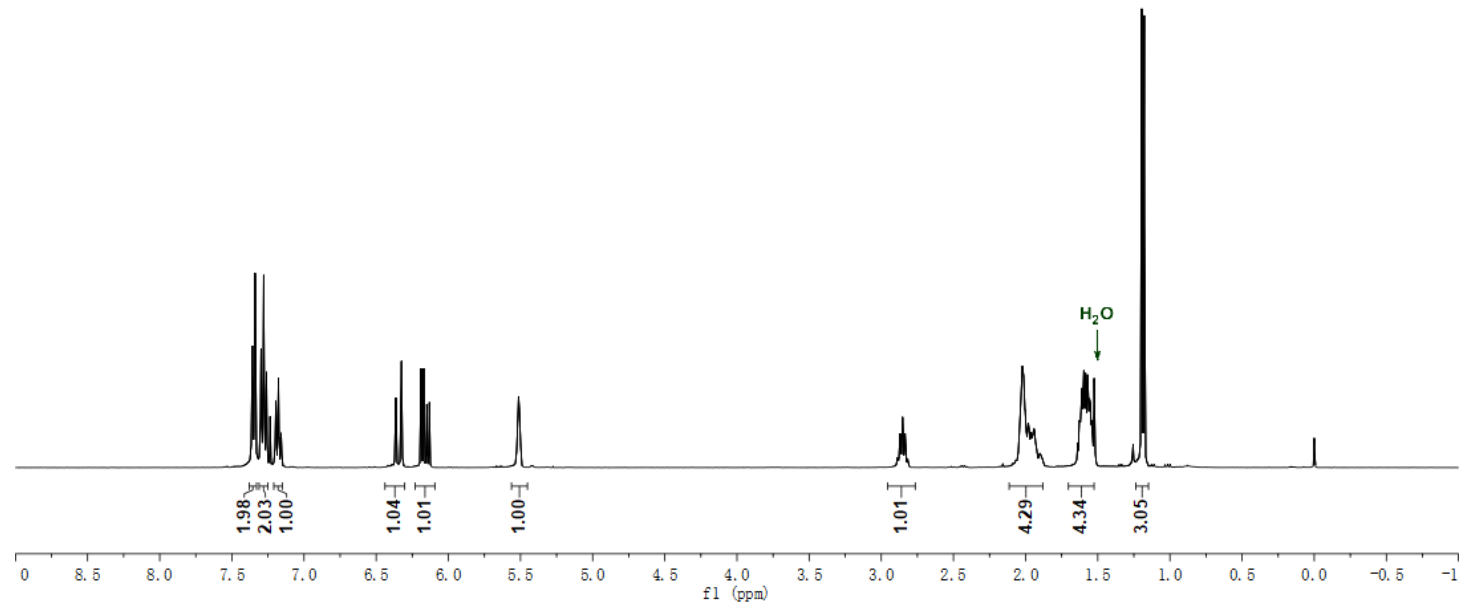

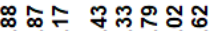

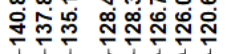

مِ:

事
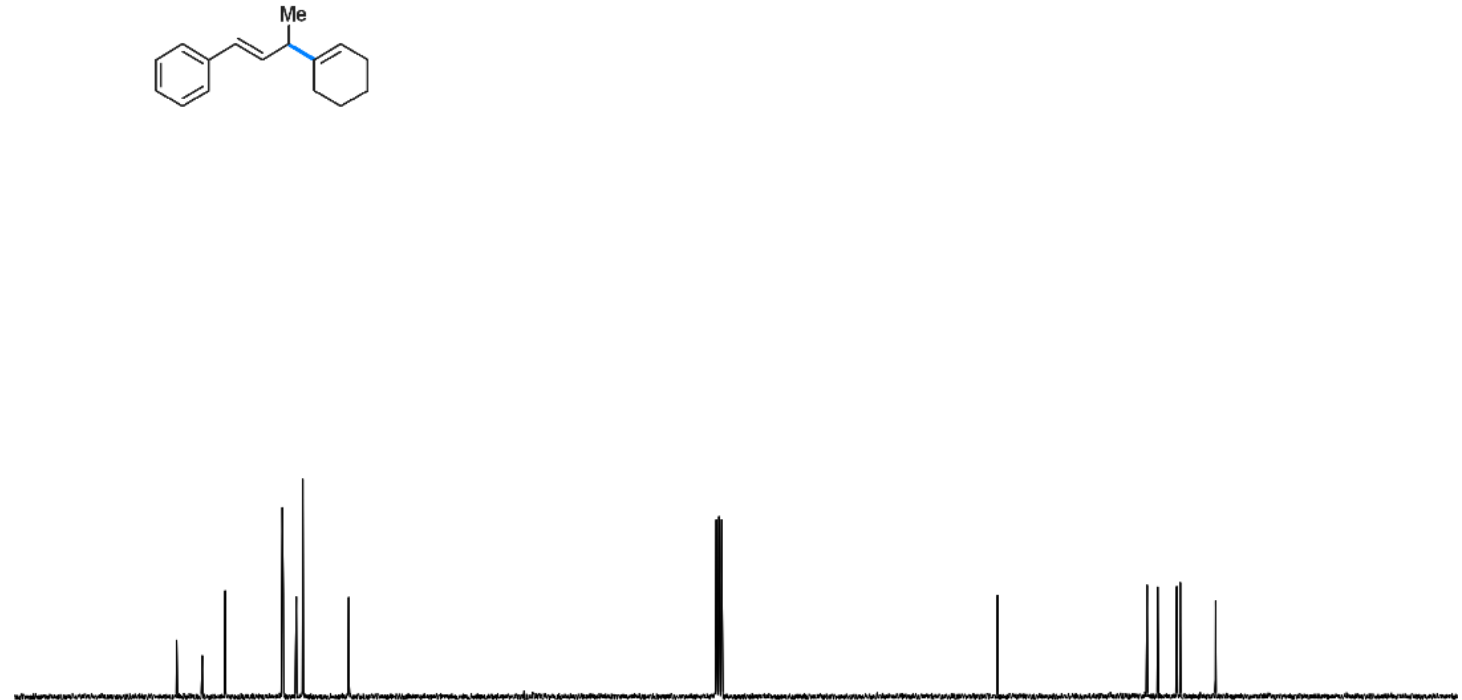

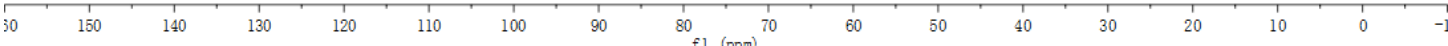


3o; ${ }^{1} \mathrm{H}$ NMR (400MHz, $\left.\mathrm{CDCl}_{3}\right) ;{ }^{13} \mathrm{C}$ NMR (100 MHz, $\left.\mathrm{CDCl}_{3}\right)$

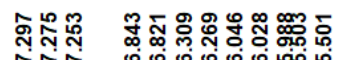

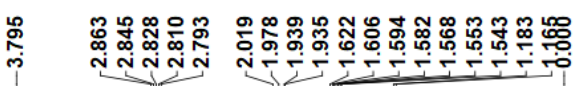<smiles>COc1ccc(/C=C/C(C)C2=CCCCC2)cc1</smiles>

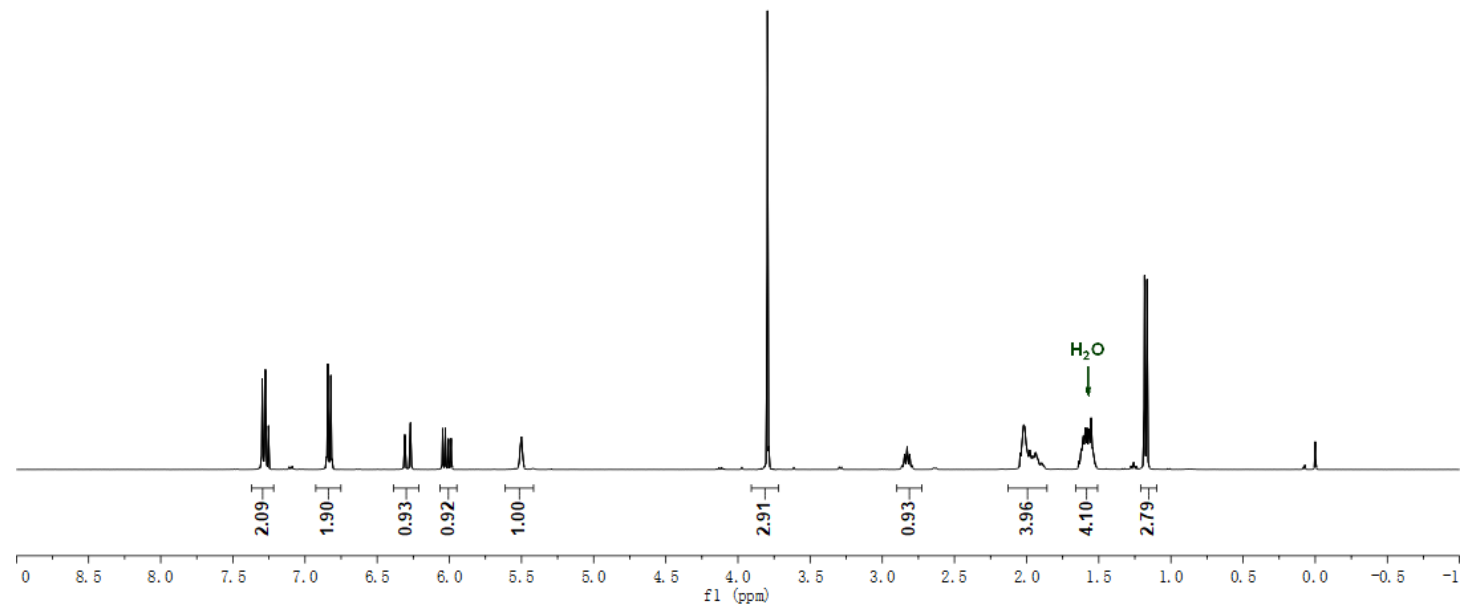

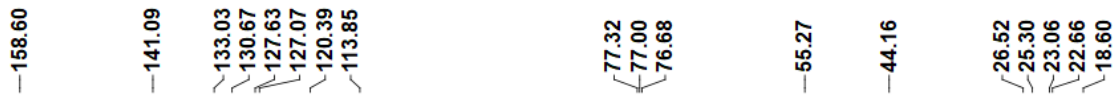<smiles>COc1ccc(/C=C/C(C)C2=CCCCC2)cc1</smiles>

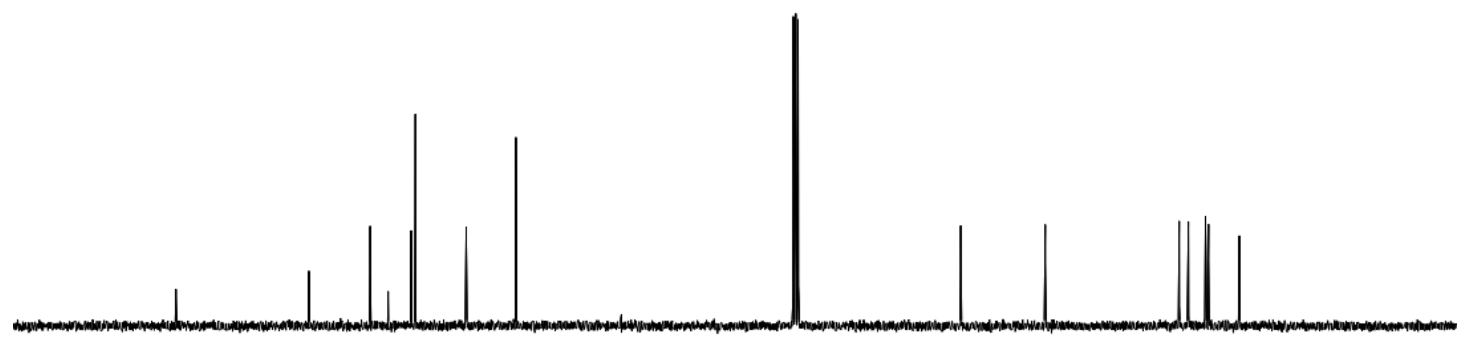

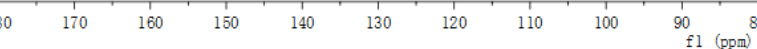


3p; ${ }^{1} \mathrm{H}$ NMR (400MHz, $\left.\mathrm{CDCl}_{3}\right) ;{ }^{13} \mathrm{C}$ NMR $\left(100 \mathrm{MHz}, \mathrm{CDCl}_{3}\right)$

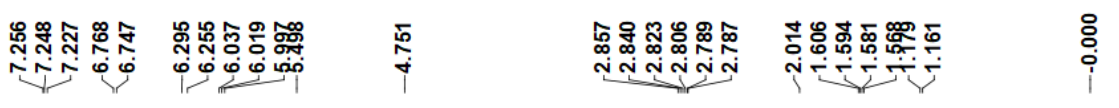<smiles>CC(C)(C=Cc1ccc(O)cc1)C1CCCCC1</smiles>

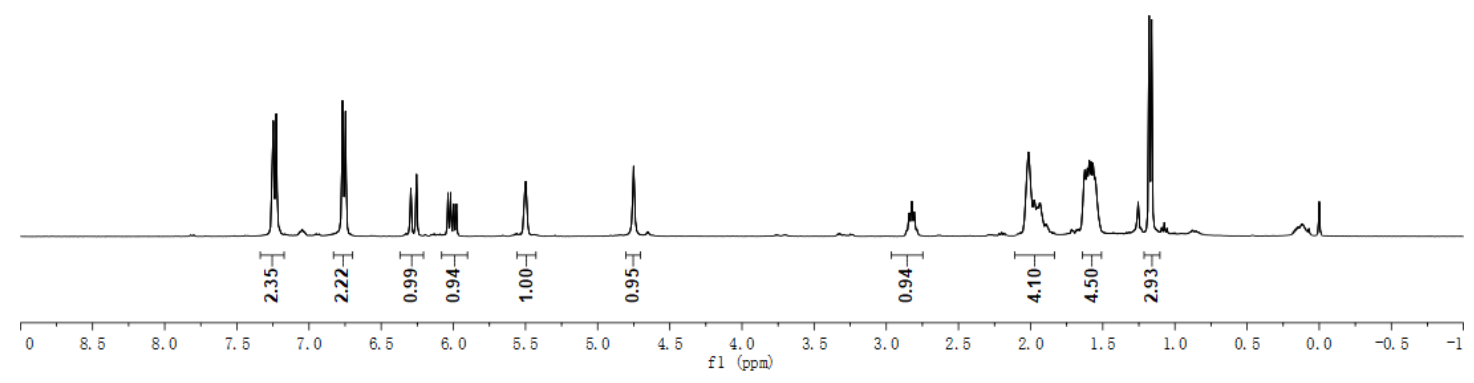

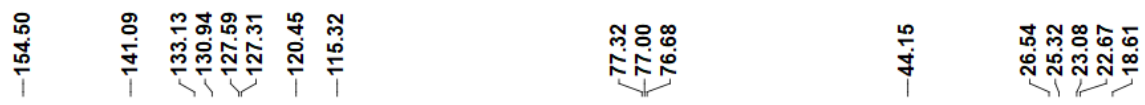<smiles>[Y16]C1=CCCCC1/C=C/c1ccc(O)cc1</smiles>
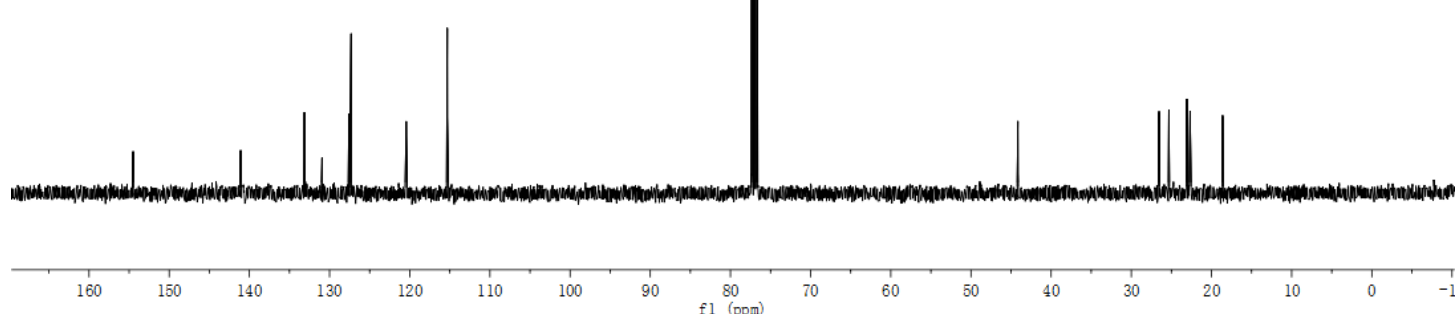
3q; ${ }^{1} \mathrm{H}$ NMR (400MHz, $\left.\mathrm{CDCl}_{3}\right) ;{ }^{13} \mathrm{C}$ NMR (100 MHz, $\left.\mathrm{CDCl}_{3}\right)$

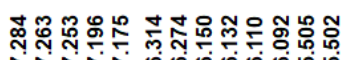

个分公

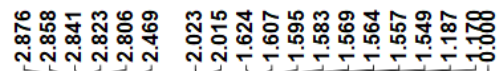

Mes

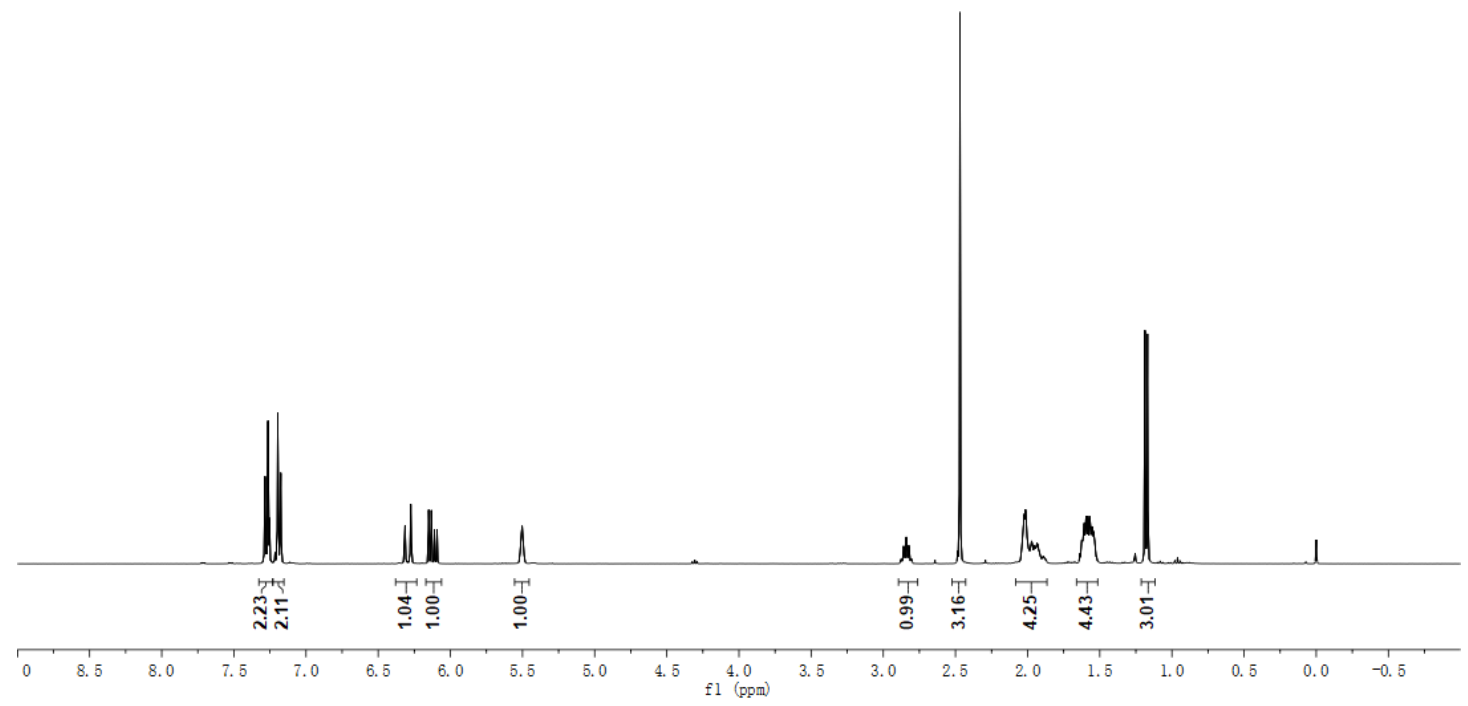

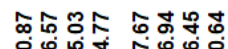

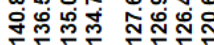

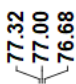

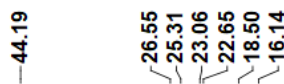
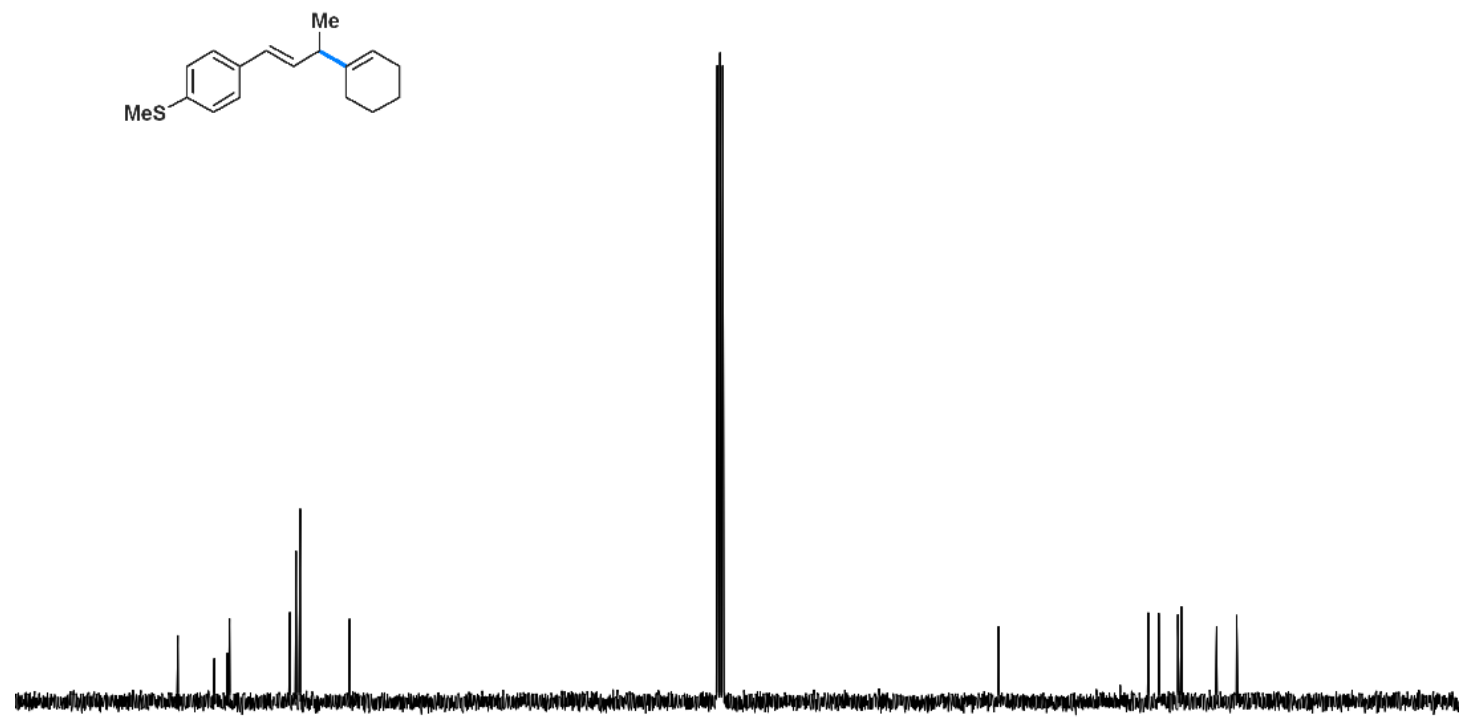

$120 \quad 11$

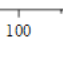

f1 (ppm) ${ }^{70}$

50

$40 \quad 30$

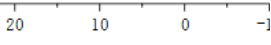

S108 
3r; ${ }^{1} \mathrm{H}$ NMR (400MHz, $\left.\mathrm{CDCl}_{3}\right) ;{ }^{13} \mathrm{C}$ NMR (100 MHz, $\left.\mathrm{CDCl}_{3}\right)$
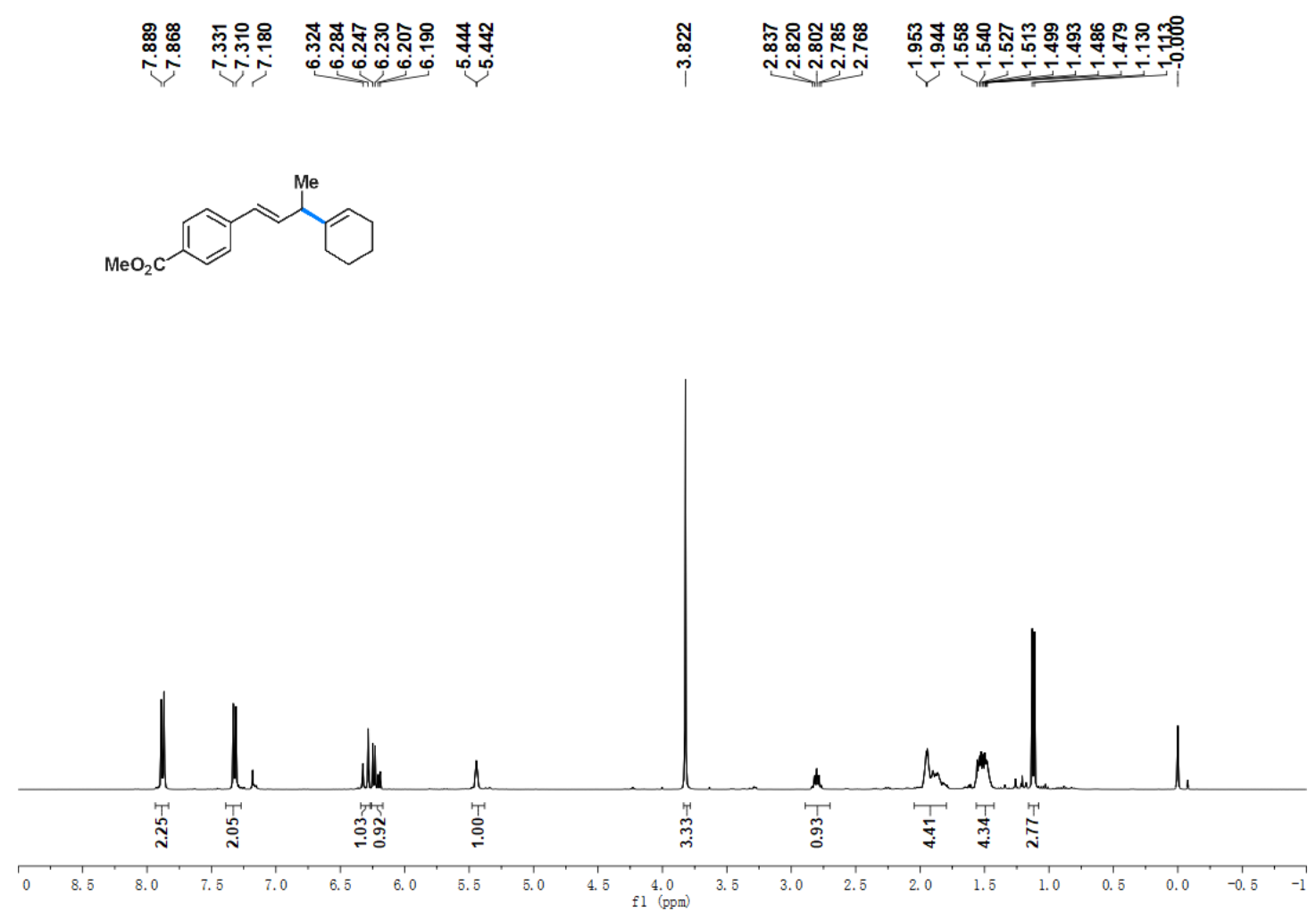

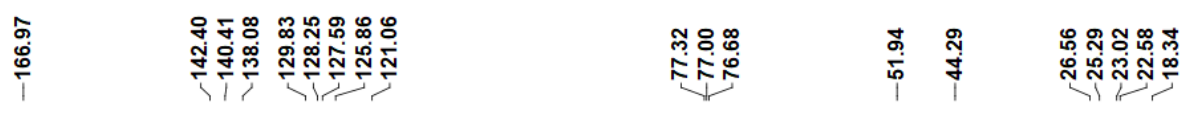<smiles>COc1ccc(/C=C/C(C)C2=CCCCC2)cc1</smiles>
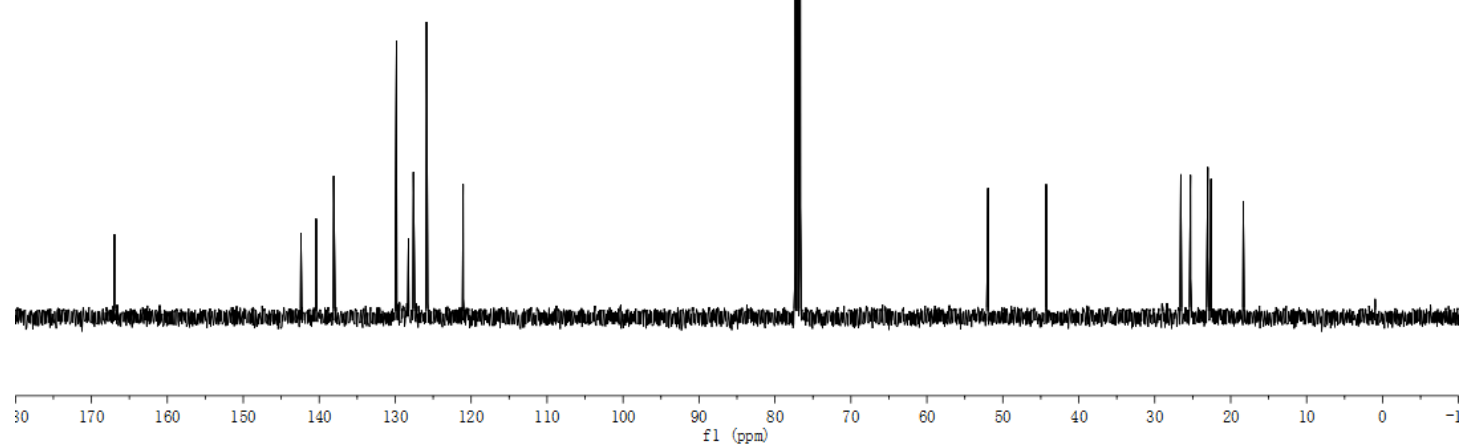
3s; ${ }^{1} \mathrm{H}$ NMR (400MHz, $\left.\mathrm{CDCl}_{3}\right) ;{ }^{13} \mathrm{C}$ NMR (100 MHz, $\left.\mathrm{CDCl}_{3}\right)$

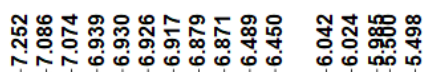

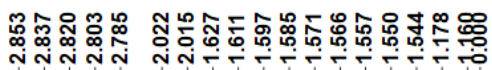
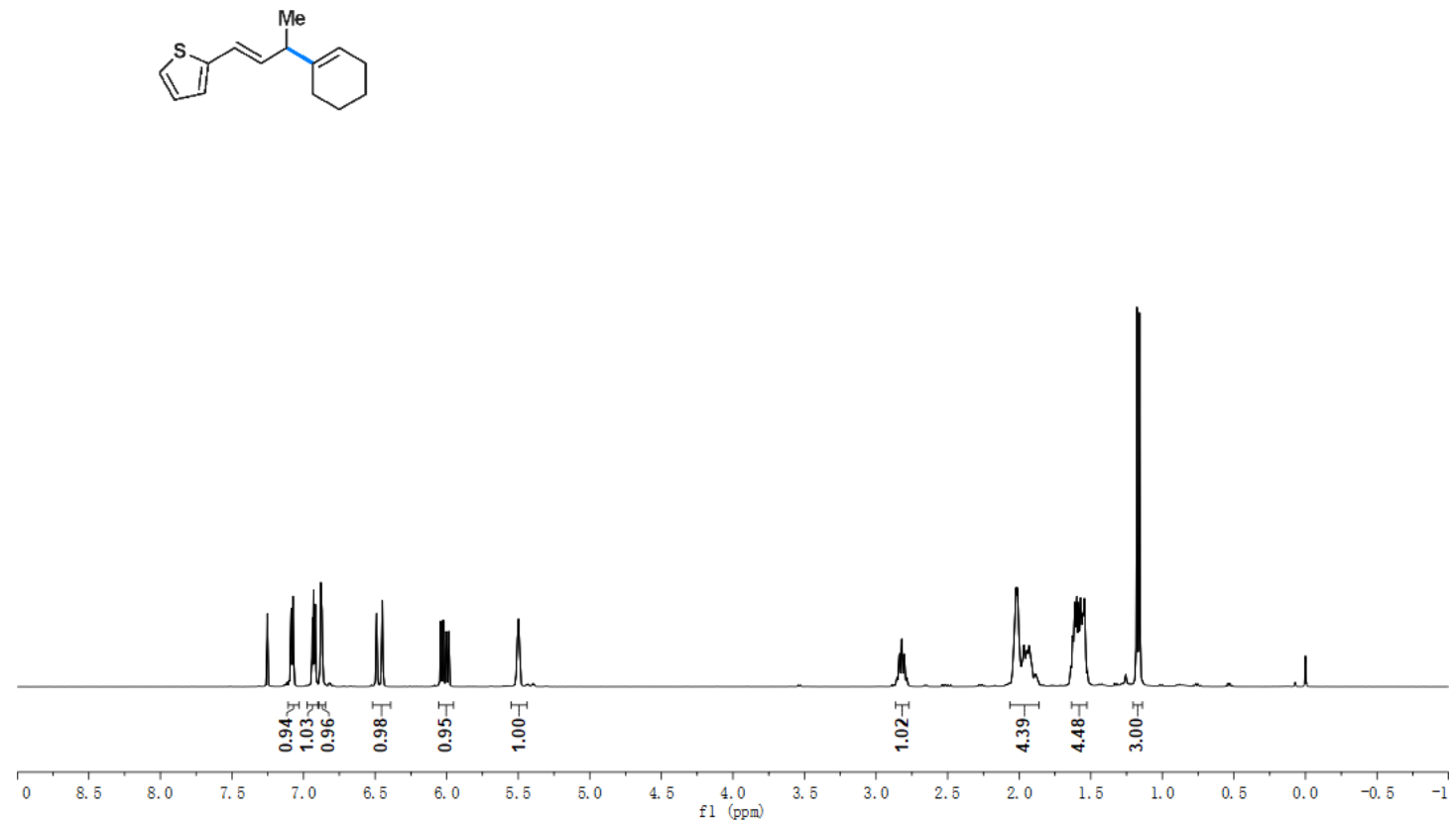

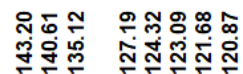

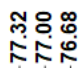

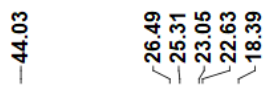
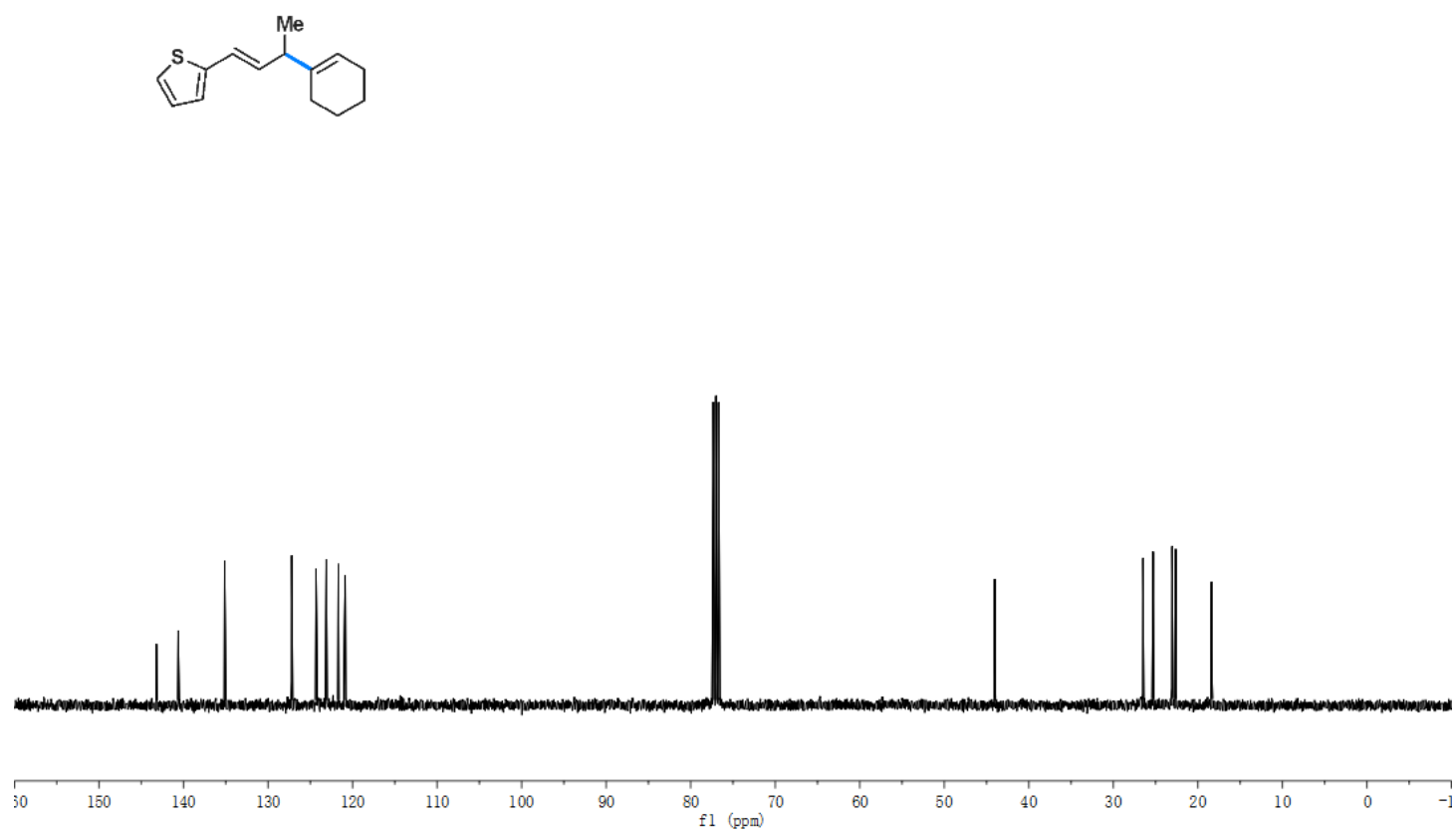

$\mathrm{S} 110$ 
3t; ${ }^{1} \mathrm{H}$ NMR (600MHz, $\left.\mathrm{CDCl}_{3}\right) ;{ }^{13} \mathrm{C}$ NMR (100 MHz, $\left.\mathrm{CDCl}_{3}\right)$

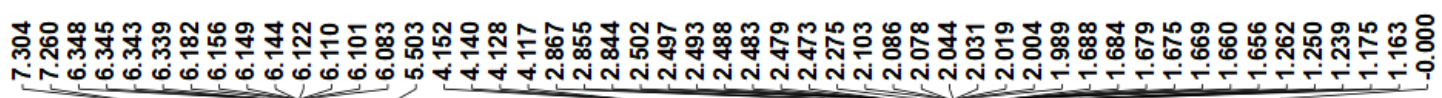<smiles>CCOC1CC=C(C(C)(C)/C=C/c2ccco2)CC1</smiles>

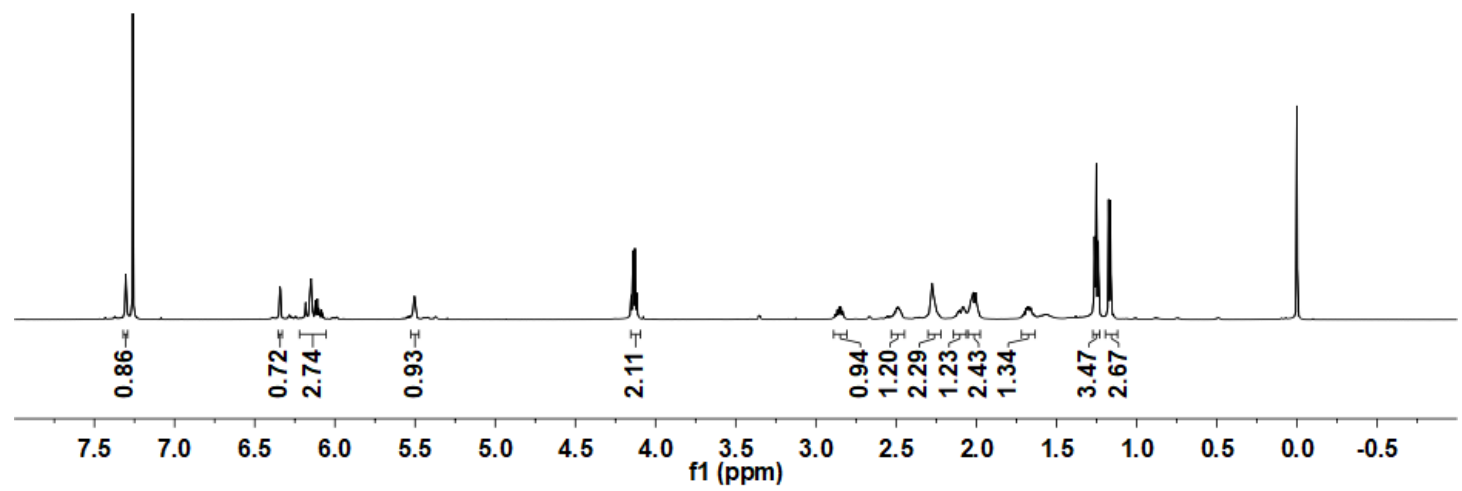

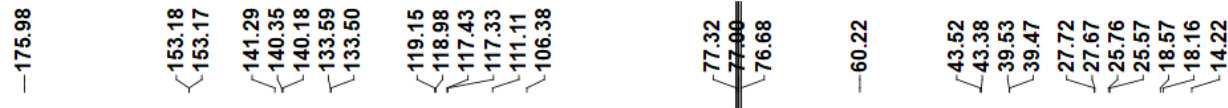<smiles>CCOC1CC=C([C@@](C)(O)/C=C/c2ccco2)CC1</smiles>

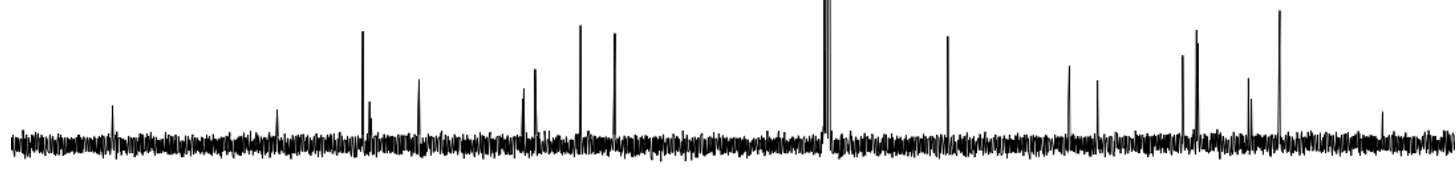

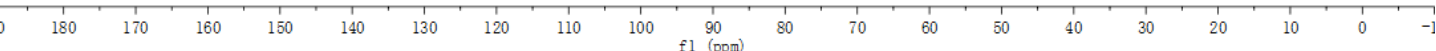


3u; ${ }^{1} \mathrm{H}$ NMR (400MHz, $\left.\mathrm{CDCl}_{3}\right) ;{ }^{13} \mathrm{C}$ NMR (100 MHz, $\left.\mathrm{CDCl}_{3}\right)$

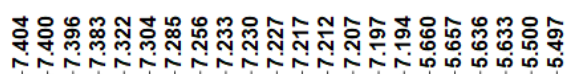

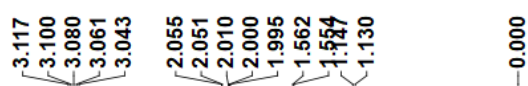
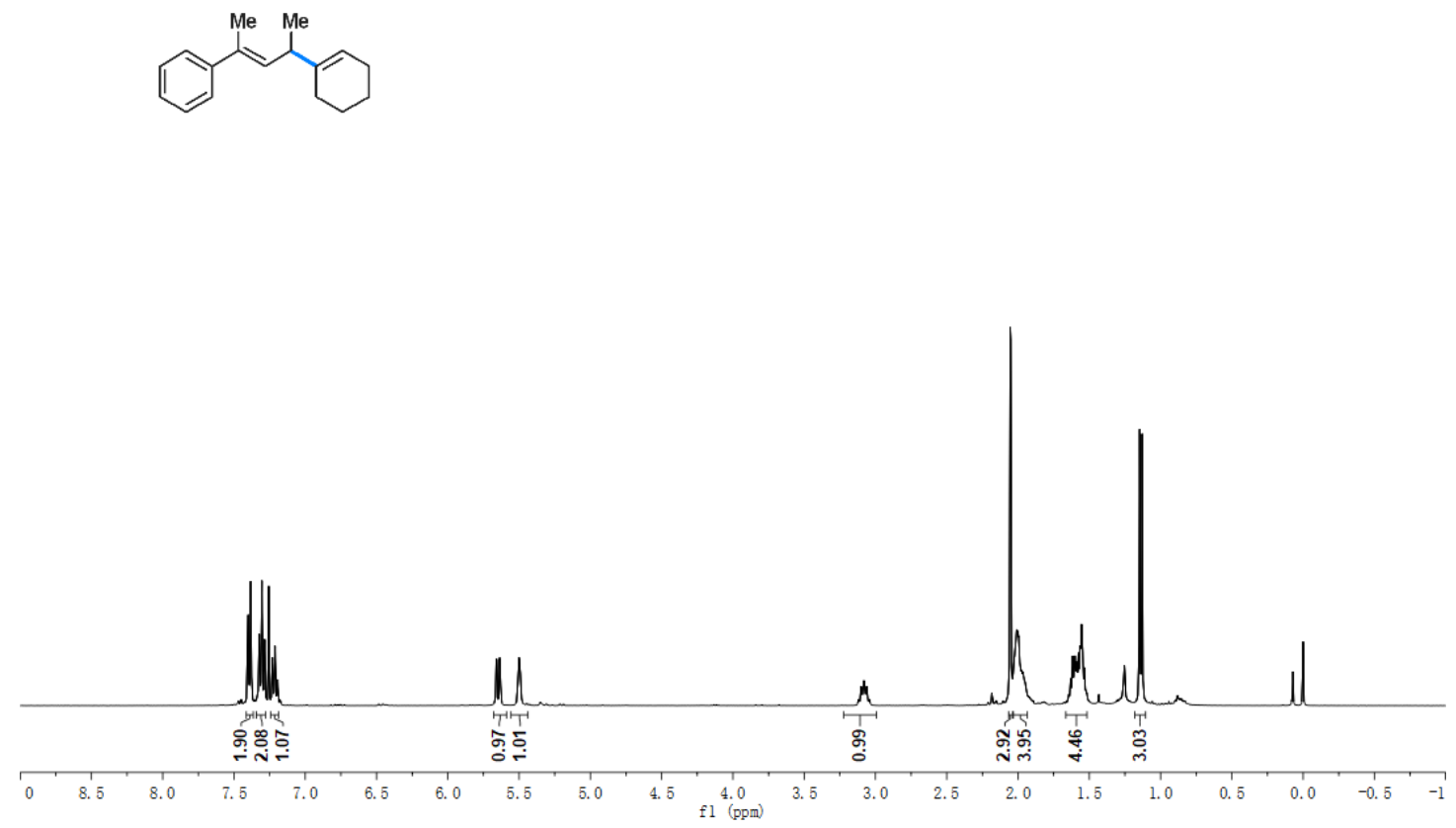

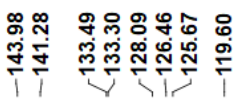

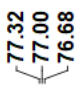

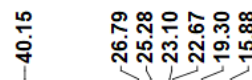
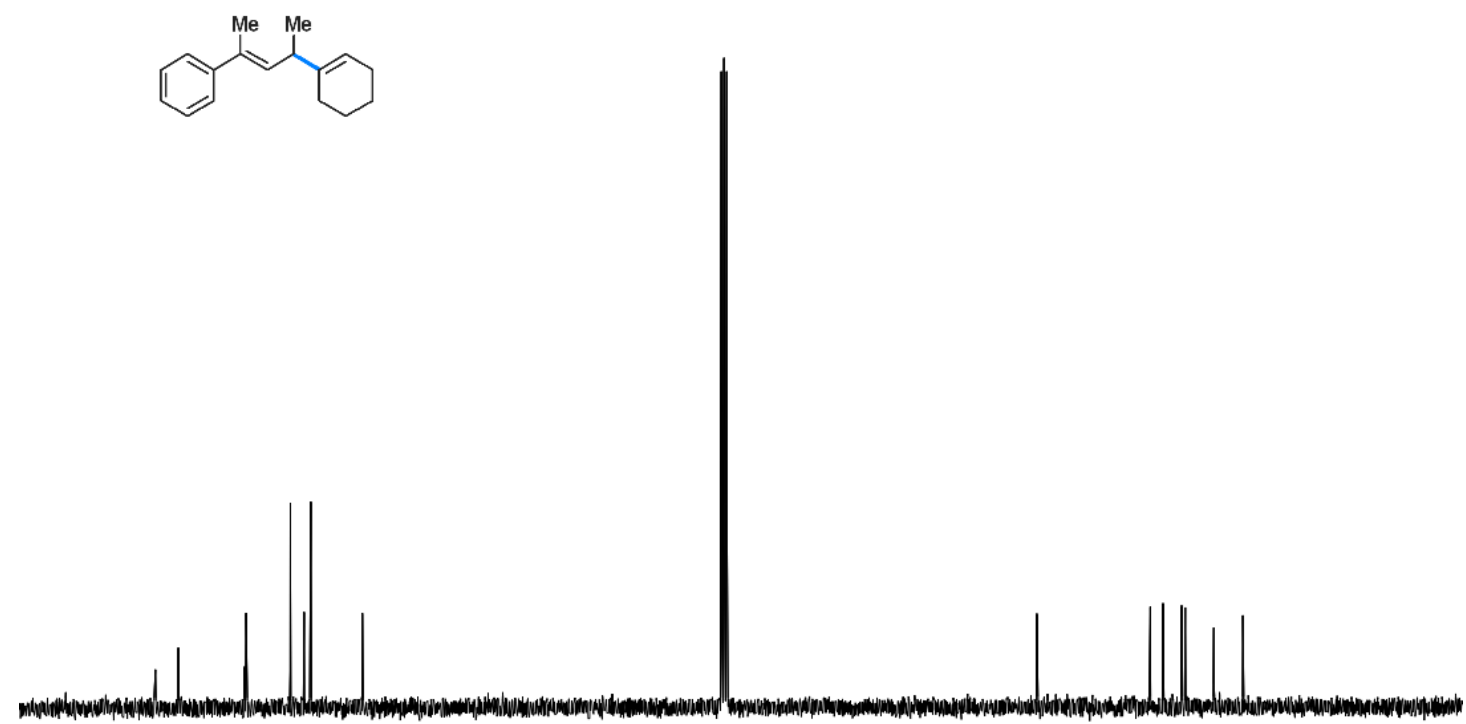

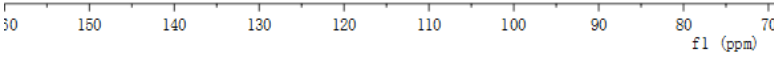


3v; ${ }^{1} \mathrm{H}$ NMR (400MHz, $\left.\mathrm{CDCl}_{3}\right) ;{ }^{13} \mathrm{C}$ NMR (100 MHz, $\left.\mathrm{CDCl}_{3}\right)$

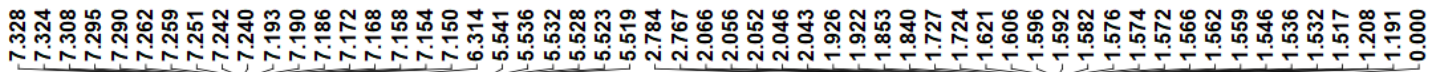
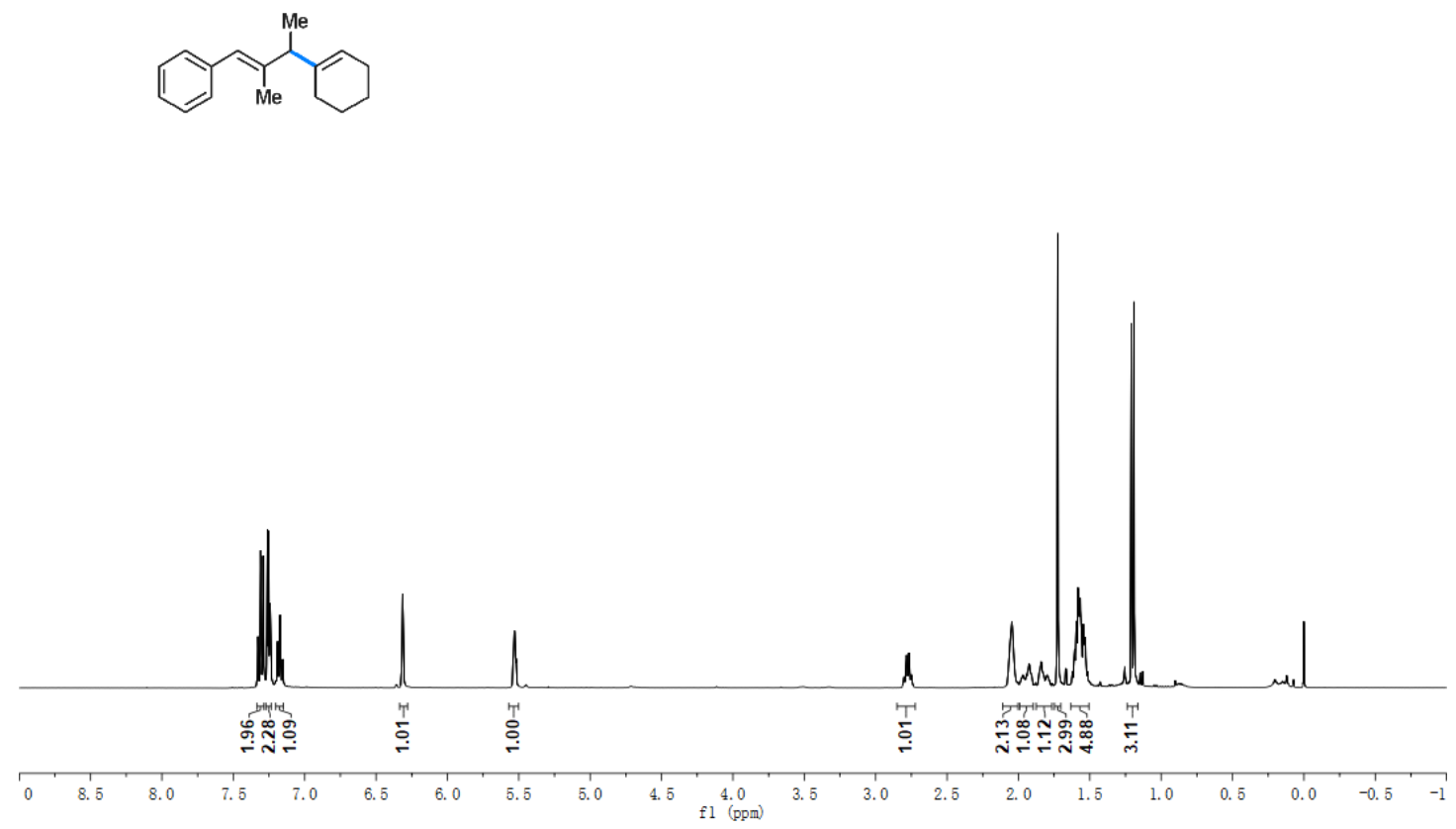

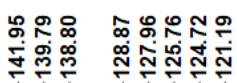

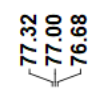

พั)

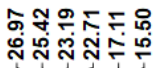
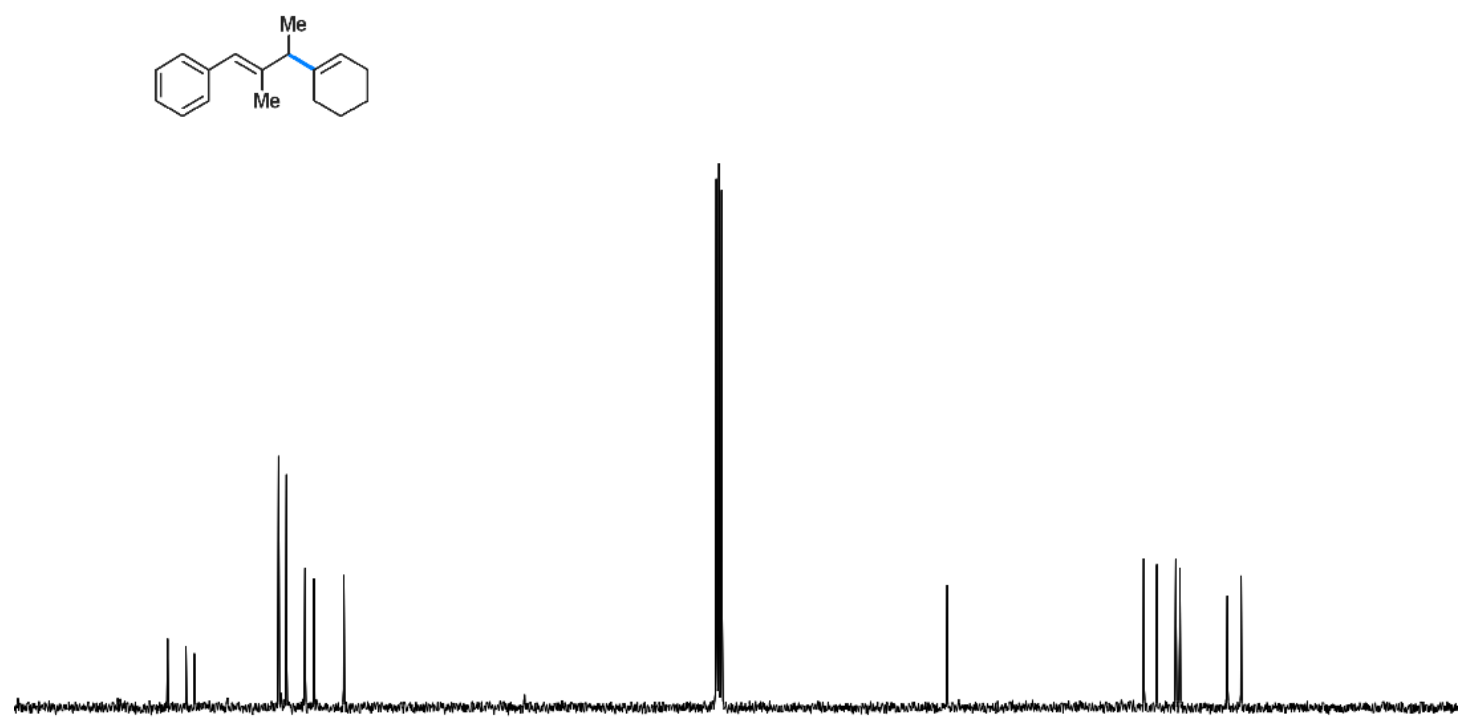

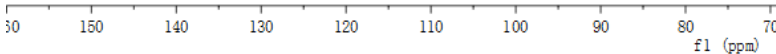


3w; ${ }^{1} \mathrm{H}$ NMR (400MHz, $\left.\mathrm{CDCl}_{3}\right) ;{ }^{13} \mathrm{C}$ NMR (100 MHz, $\left.\mathrm{CDCl}_{3}\right)$

إم

Me

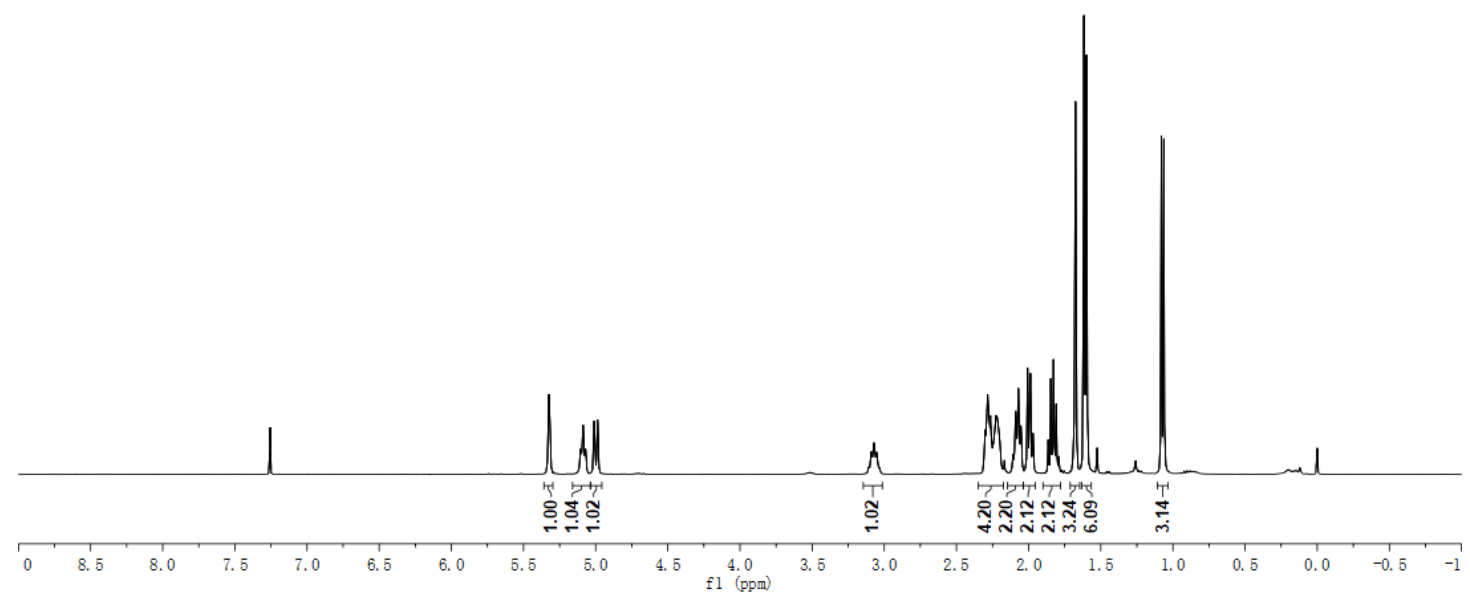

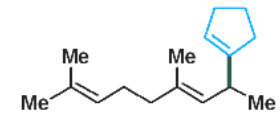

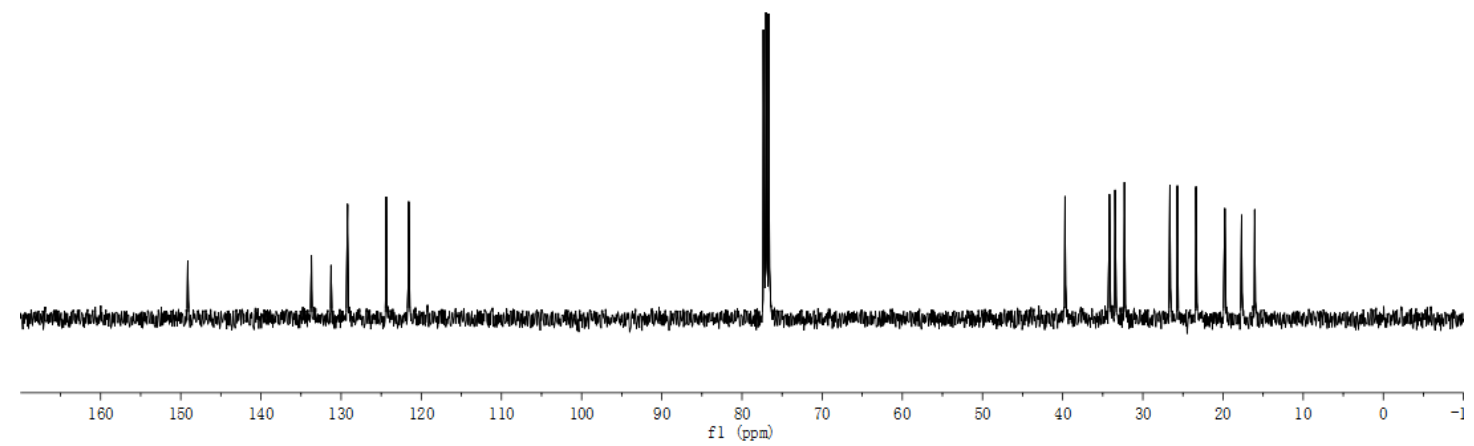


$3 \mathrm{x} ;{ }^{1} \mathrm{H}$ NMR (400MHz, $\left.\mathrm{CDCl}_{3}\right) ;{ }^{13} \mathrm{C}$ NMR (100 MHz, $\left.\mathrm{CDCl}_{3}\right)$<smiles>CC(C)=CCCc1cc(C)ccc1C</smiles>

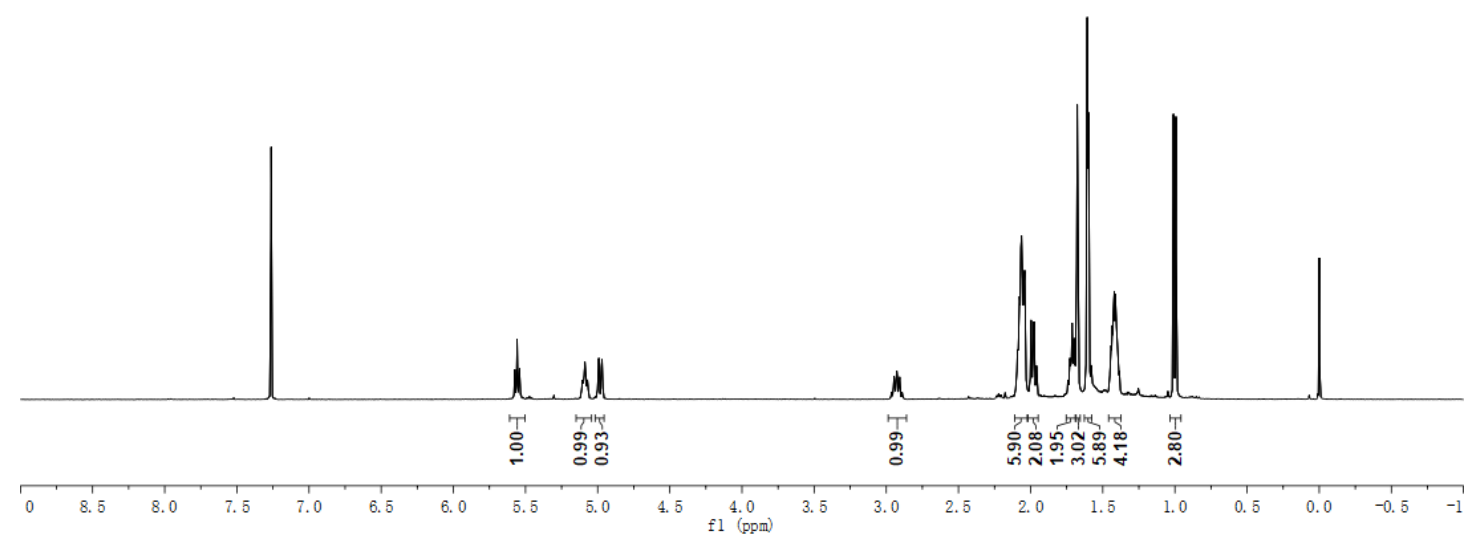

$$
\text { (l) }
$$

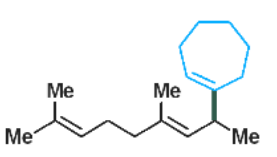

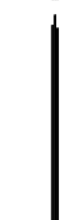


3y; ${ }^{1} \mathrm{H}$ NMR (400MHz, $\left.\mathrm{CDCl}_{3}\right) ;{ }^{13} \mathrm{C}$ NMR (100 MHz, $\left.\mathrm{CDCl}_{3}\right)$

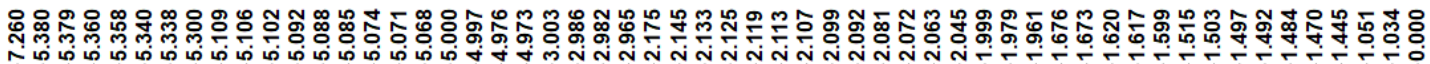
$\underbrace{\text { Me }}_{\text {Me }}$

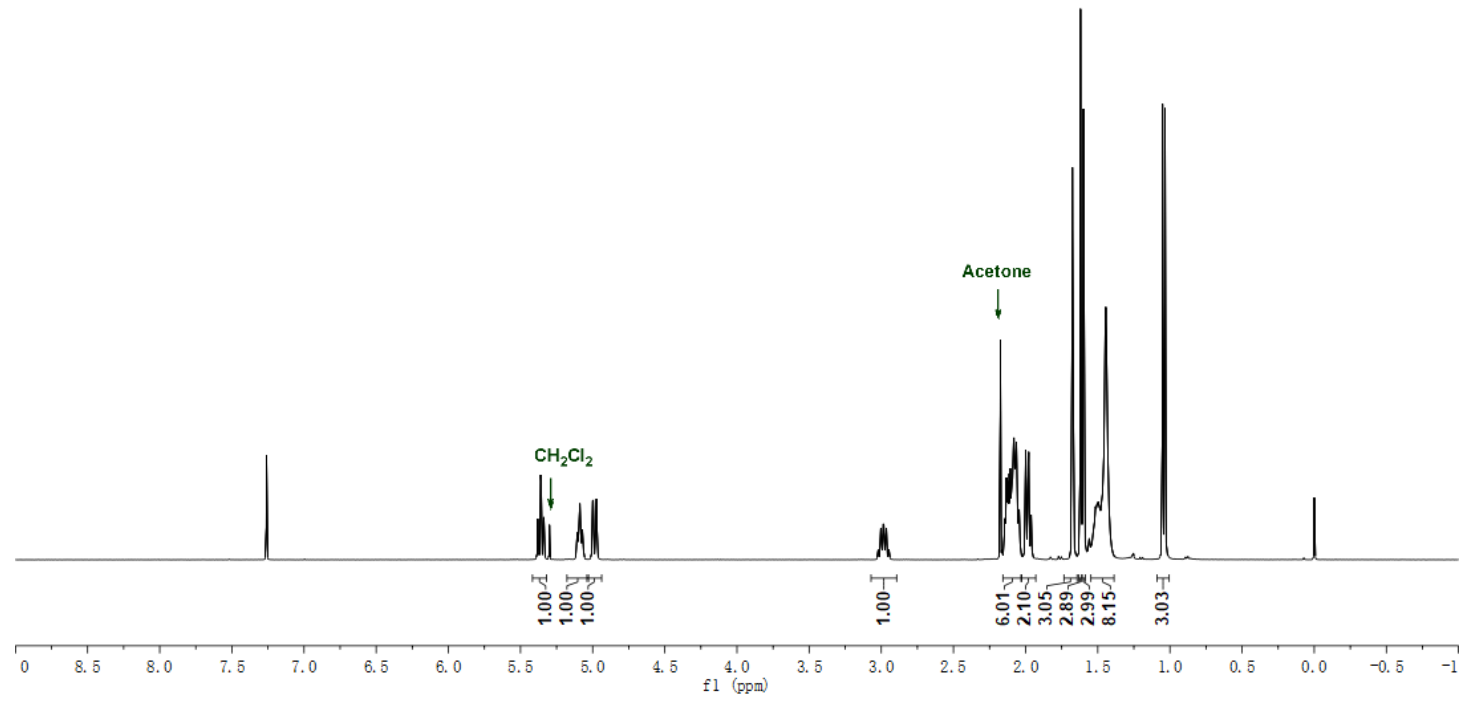

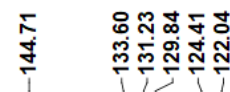

బิุ

福是

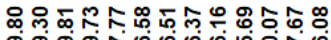

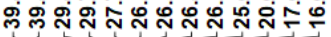
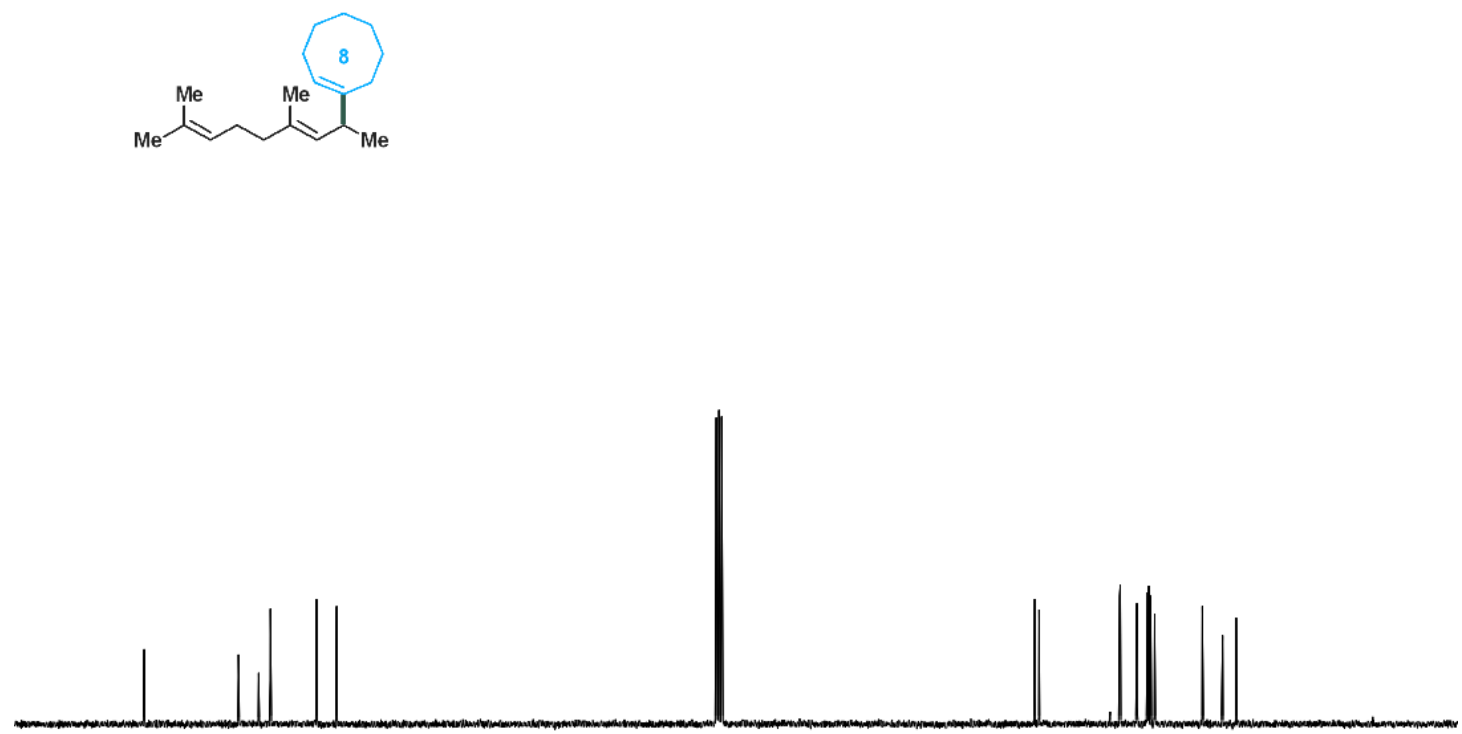

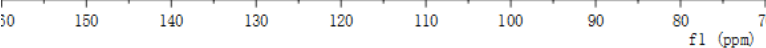


3z; ${ }^{1} \mathrm{H}$ NMR (400MHz, $\left.\mathrm{CDCl}_{3}\right) ;{ }^{13} \mathrm{C}$ NMR (100 MHz, $\left.\mathrm{CDCl}_{3}\right)$

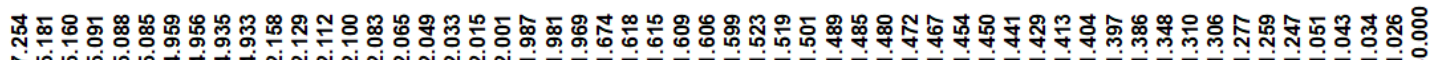

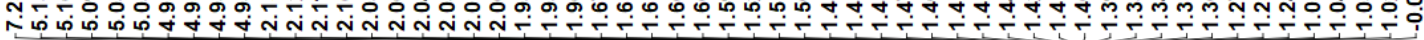

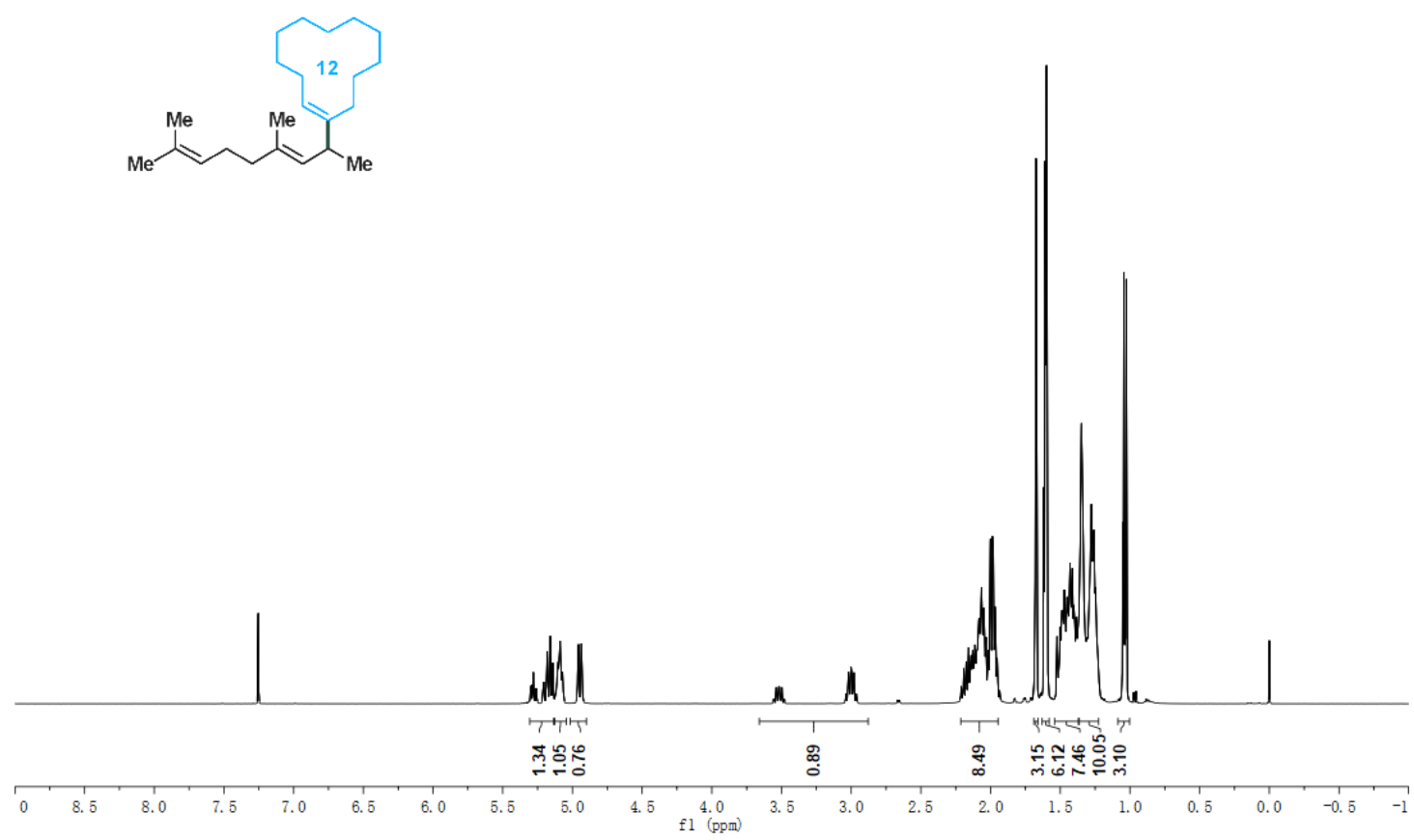

ชุำ

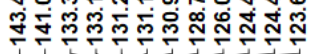

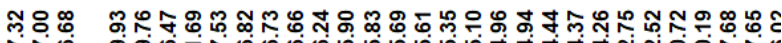

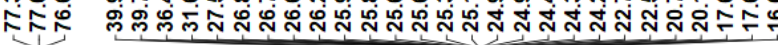

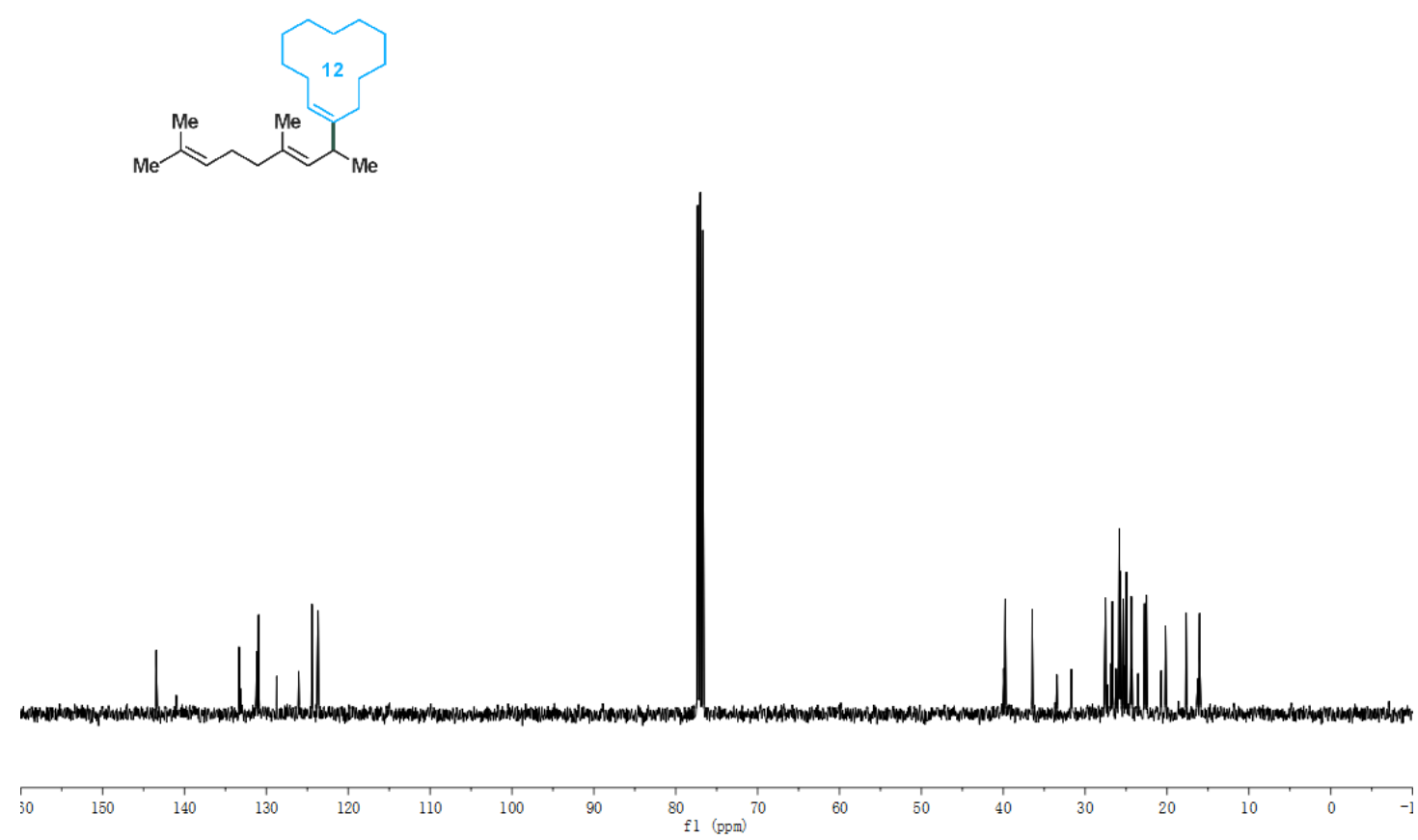


3aa; ${ }^{1} \mathrm{H}$ NMR (400MHz, $\left.\mathrm{CDCl}_{3}\right) ;{ }^{13} \mathrm{C}$ NMR (100 MHz, $\left.\mathrm{CDCl}_{3}\right)$

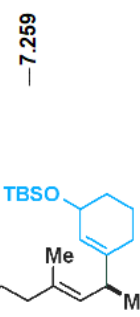

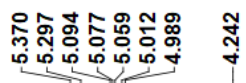

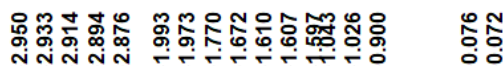

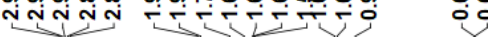

Me
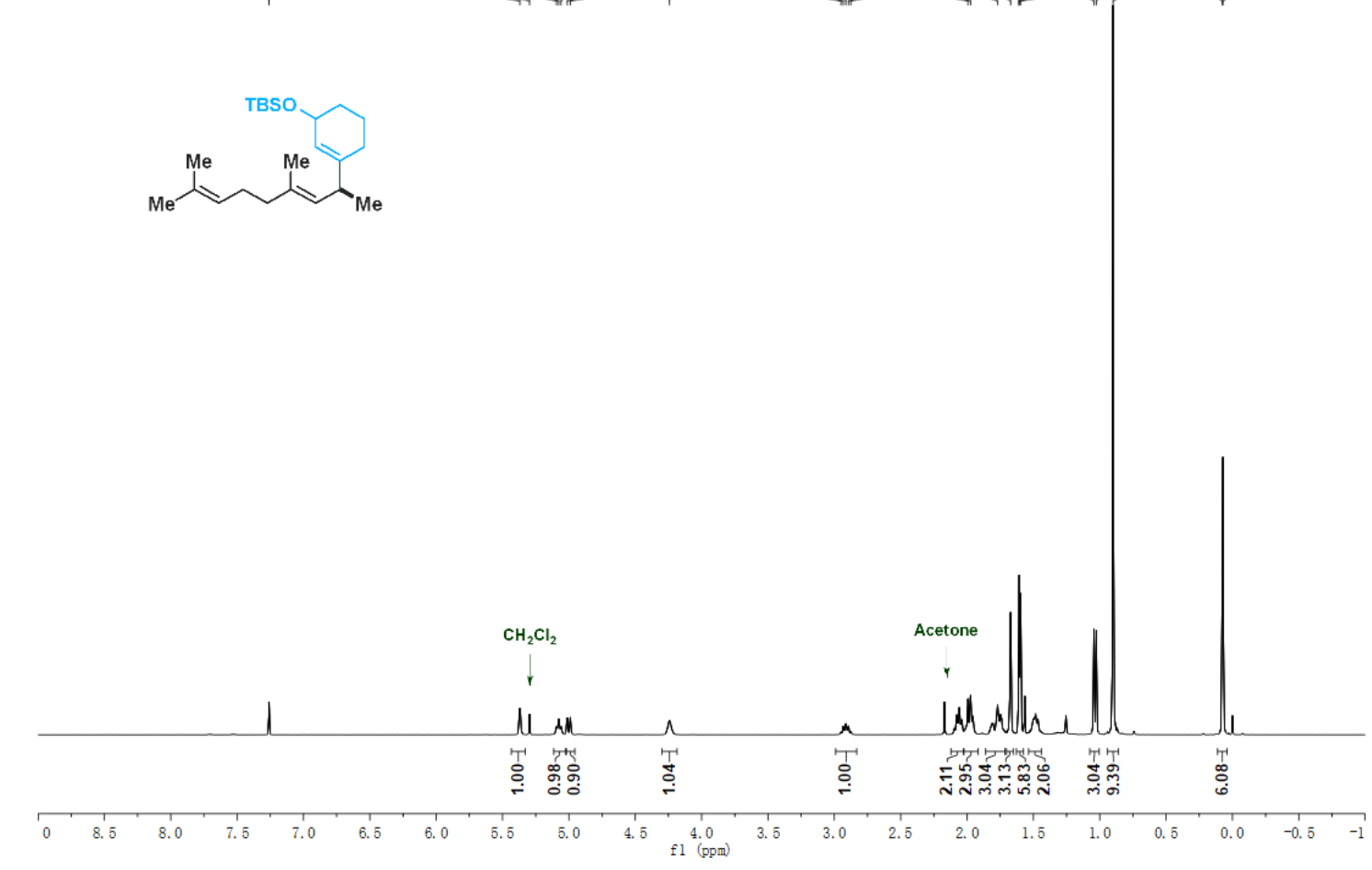

莘

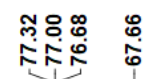

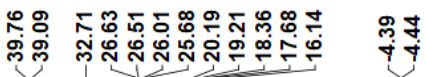
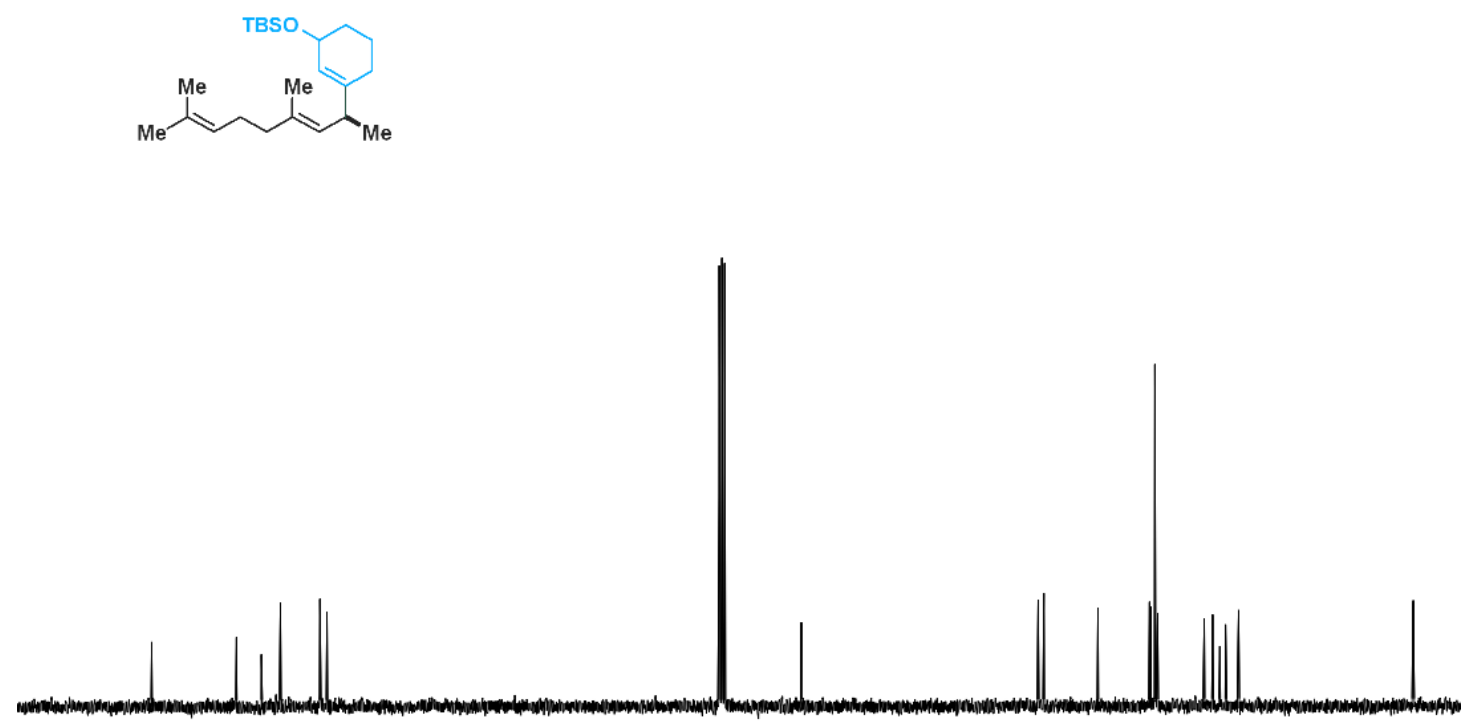

\begin{tabular}{lllllllll}
\hline 30 & 150 & 140 & 130 & 120 & 110 & 100 & 90 & 80 \\
$\mathrm{f} 11(\mathrm{ppm})$ & 7
\end{tabular} 
3ab; ${ }^{1} \mathrm{H}$ NMR (400MHz, $\left.\mathrm{CDCl}_{3}\right) ;{ }^{13} \mathrm{C}$ NMR (100 $\left.\mathrm{MHz}, \mathrm{CDCl}_{3}\right)$

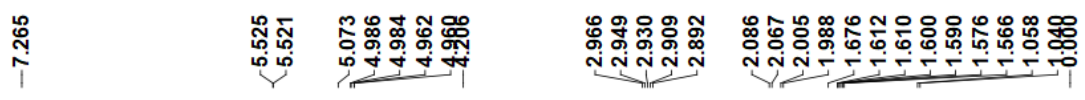

Me

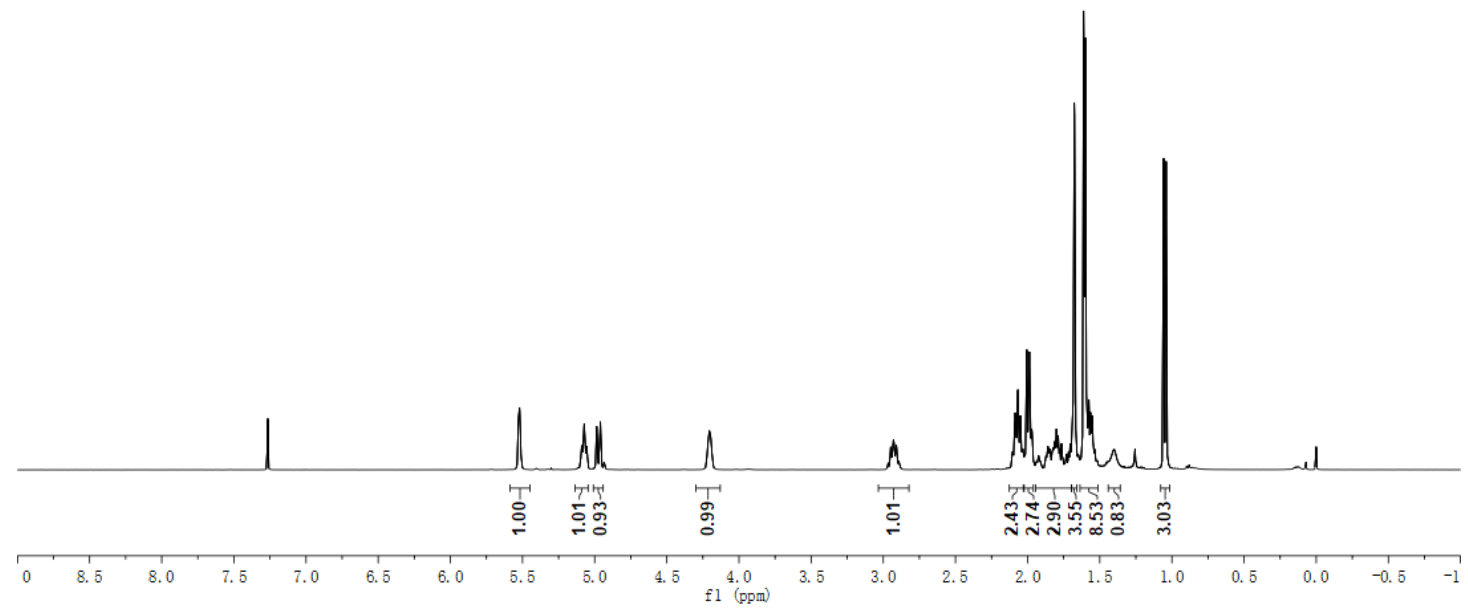

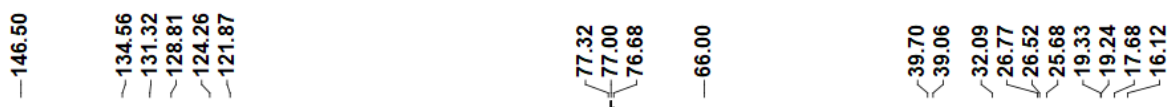
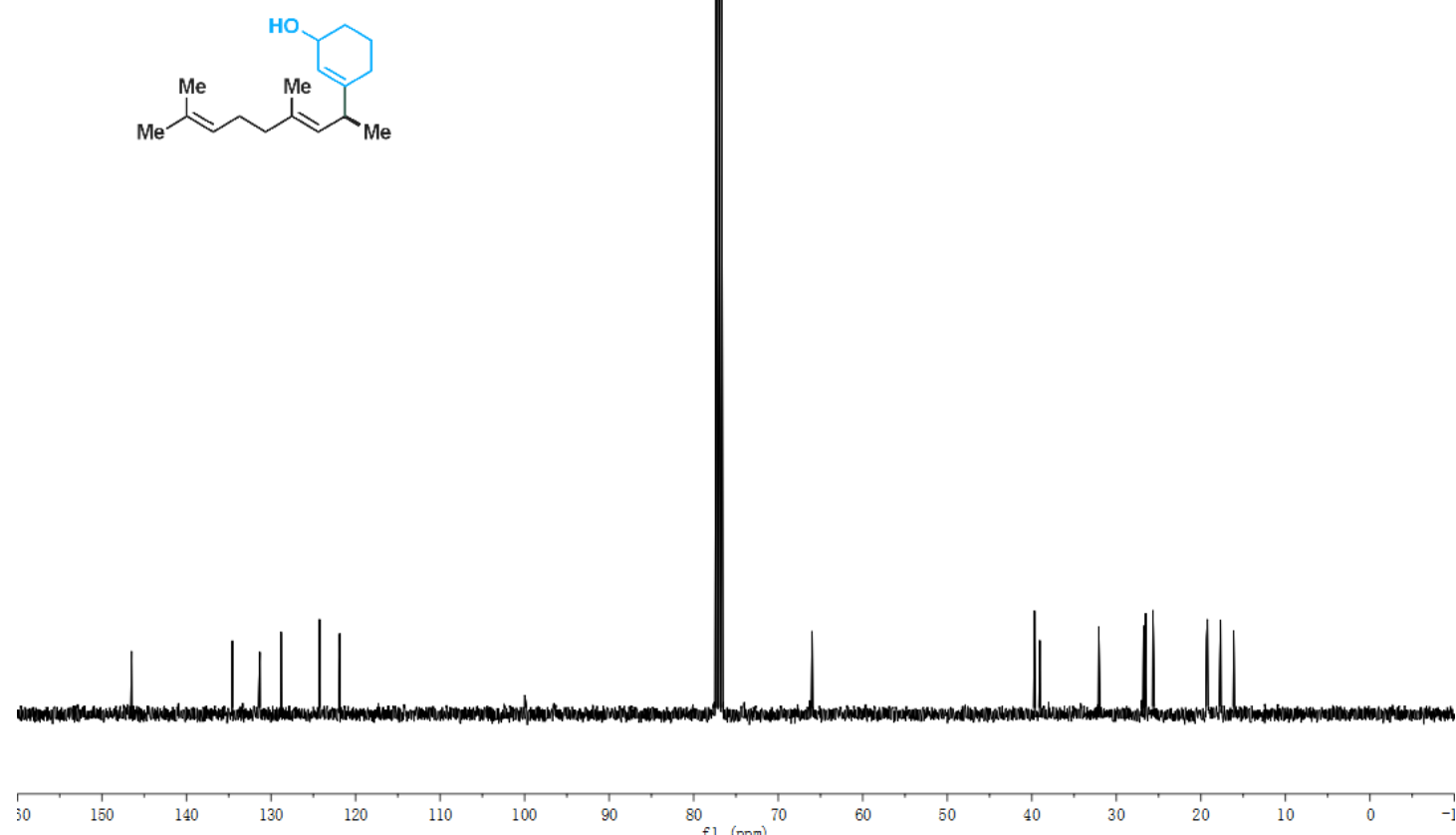
3ac; ${ }^{1} \mathrm{H}$ NMR (400MHz, $\left.\mathrm{CDCl}_{3}\right) ;{ }^{13} \mathrm{C}$ NMR $\left(100 \mathrm{MHz}, \mathrm{CDCl}_{3}\right)$

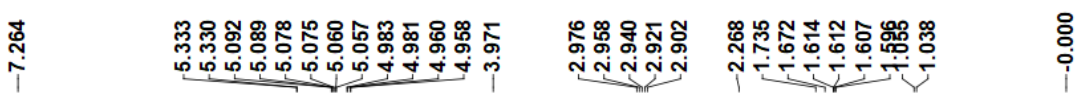

$\overbrace{M e}^{\text {Me }}$

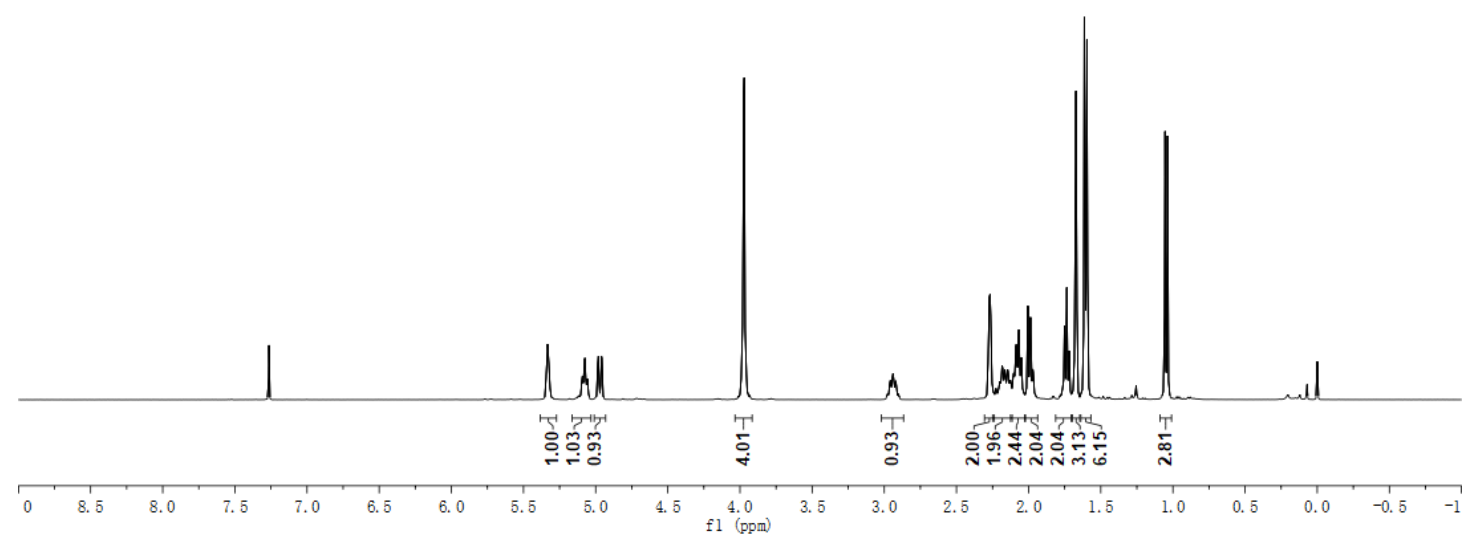

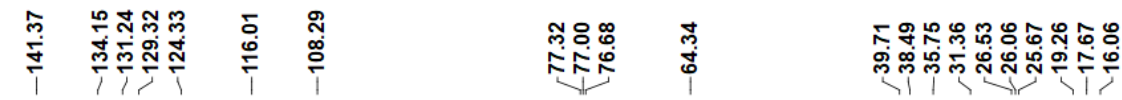
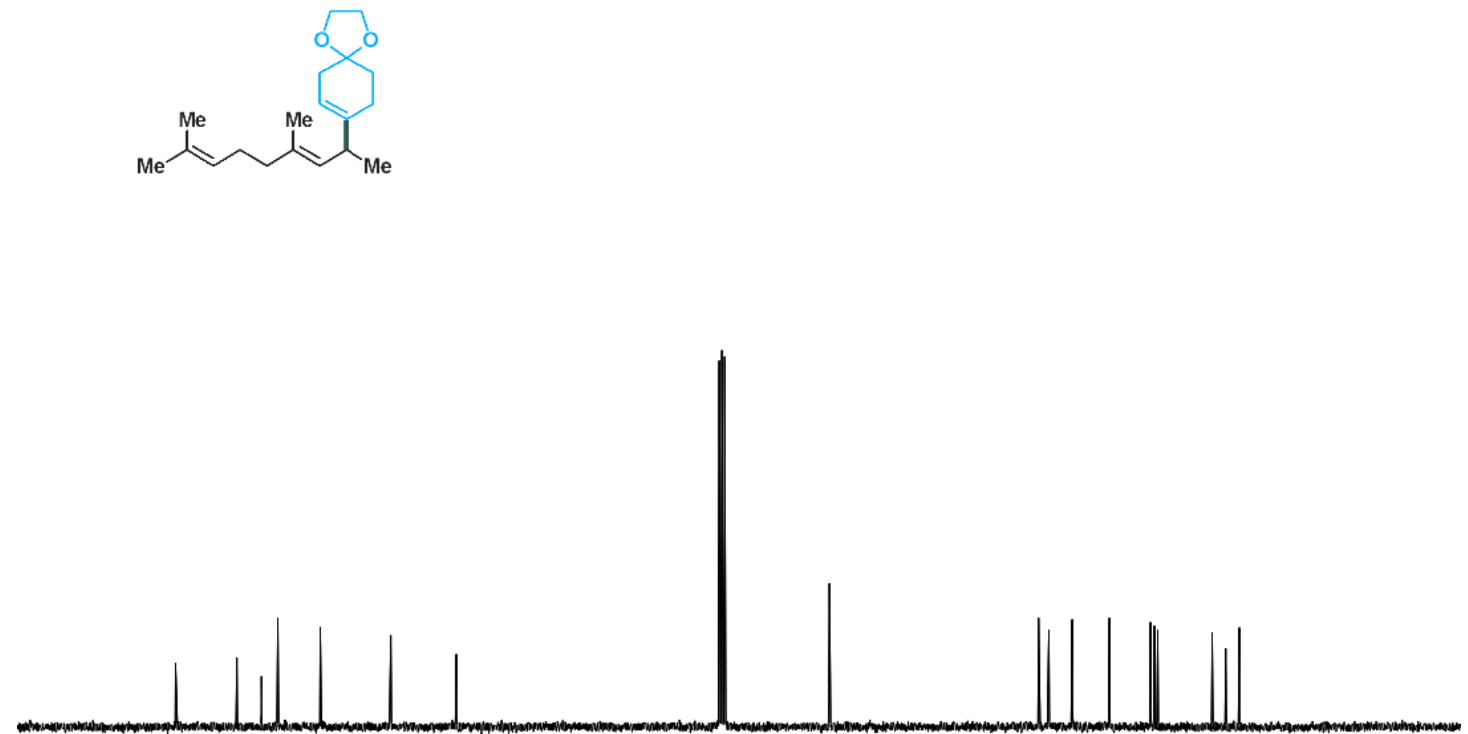

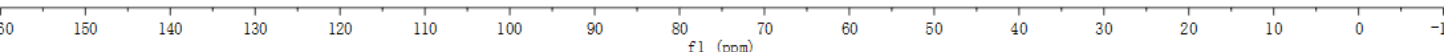




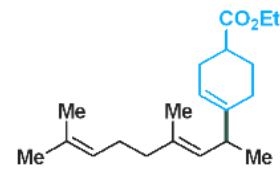

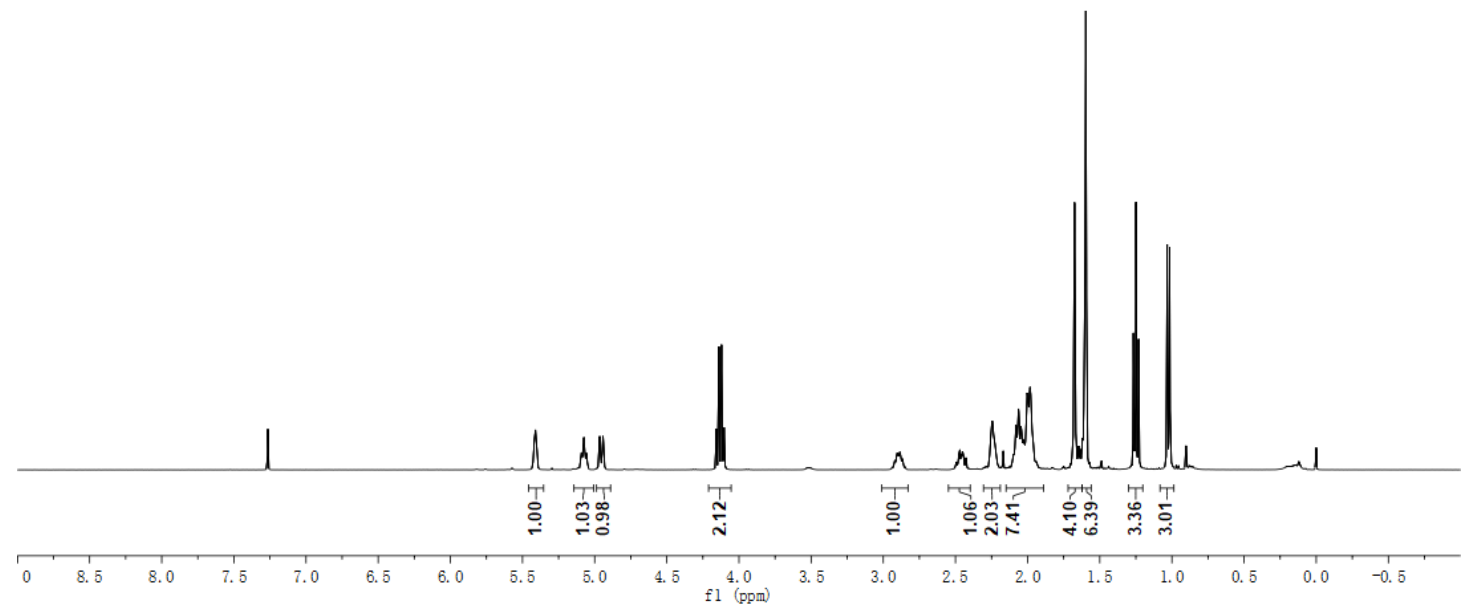

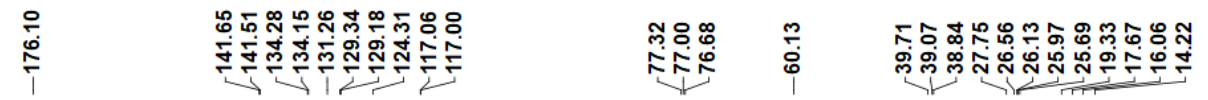<smiles>CC(C)=CCc1cc(C)cc(C)c1</smiles>

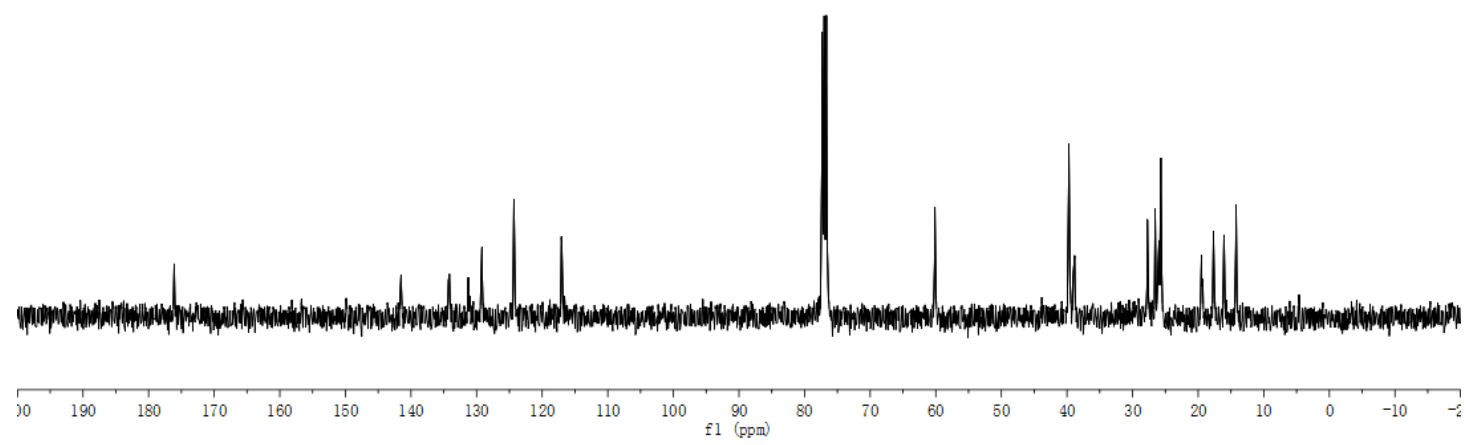


3ae; ${ }^{1} \mathrm{H}$ NMR (400MHz, $\left.\mathrm{CDCl}_{3}\right) ;{ }^{13} \mathrm{C}$ NMR (100 MHz, $\left.\mathrm{CDCl}_{3}\right)$

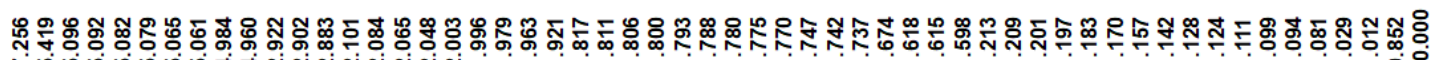
ดั

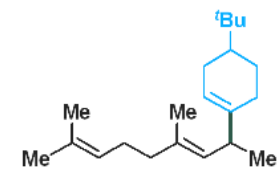

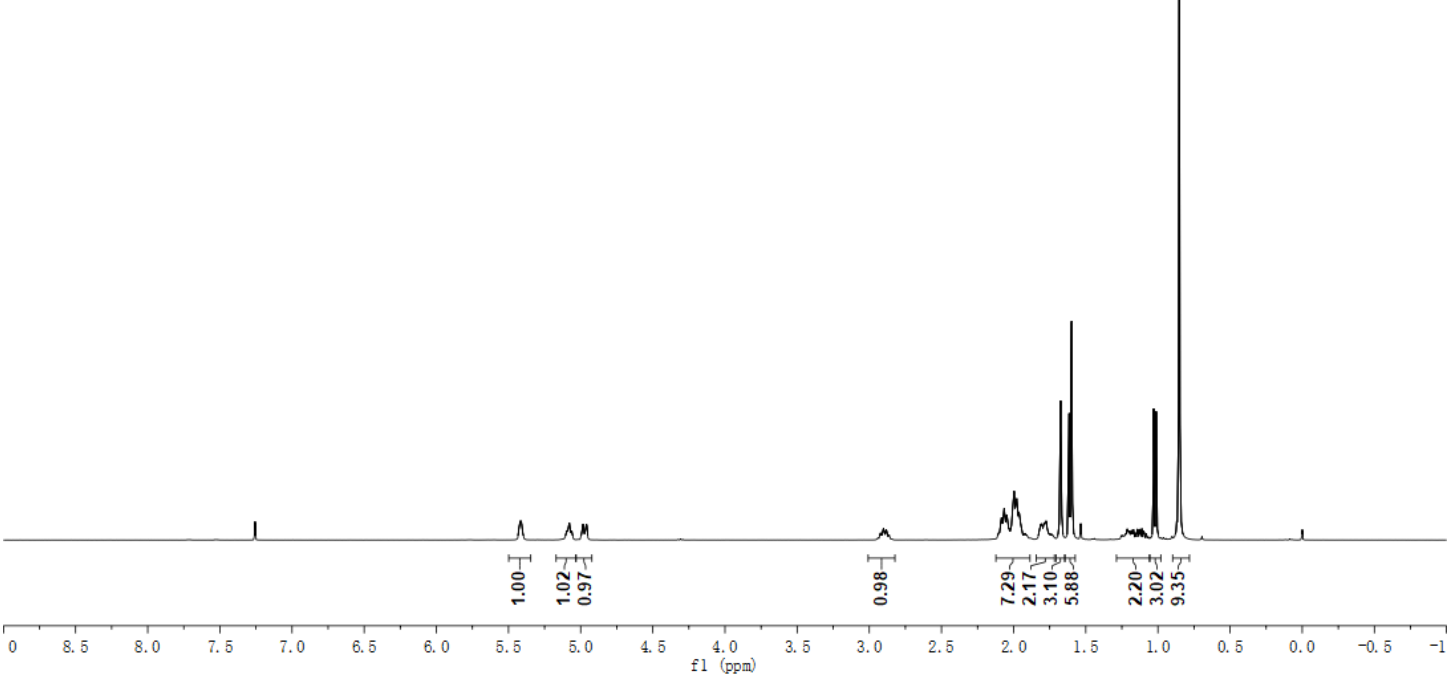

œֻ

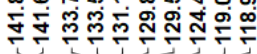
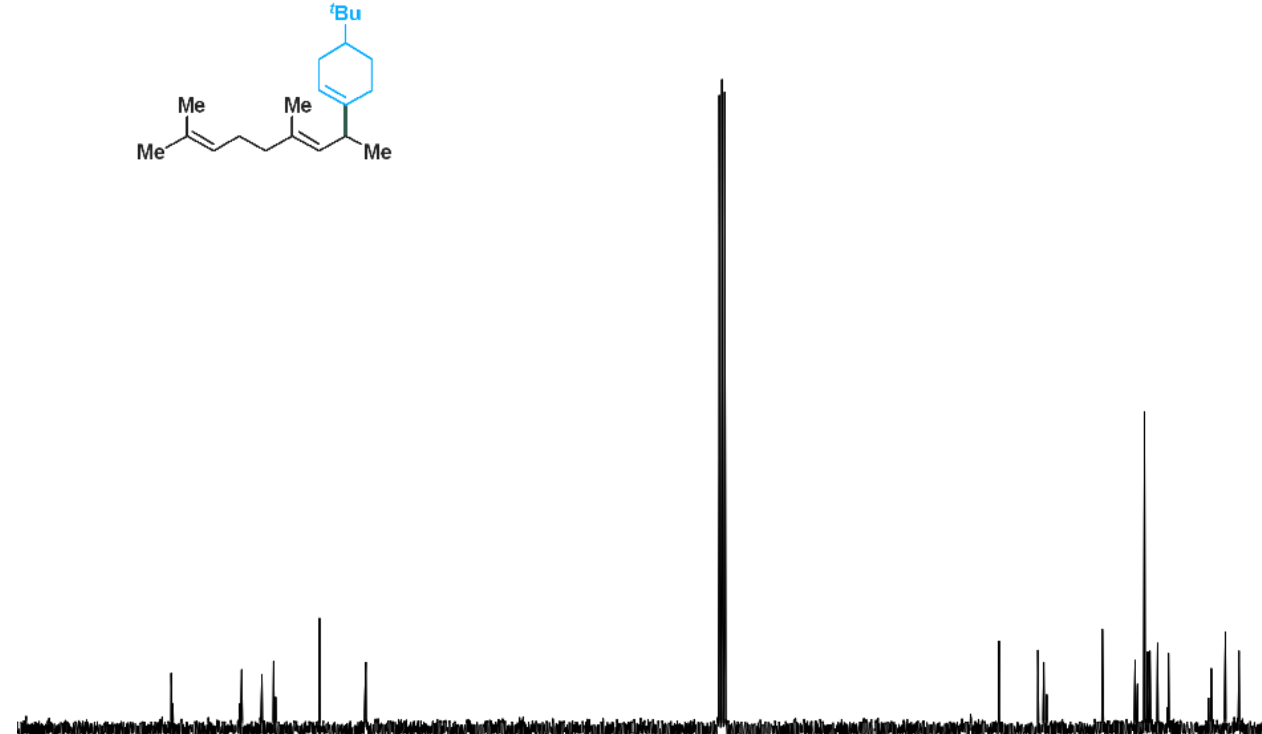

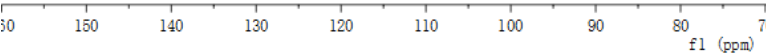


3af; ${ }^{1} \mathrm{H}$ NMR (400MHz, $\left.\mathrm{CDCl}_{3}\right) ;{ }^{13} \mathrm{C}$ NMR (100 MHz, $\left.\mathrm{CDCl}_{3}\right)$

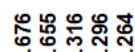

iiniti

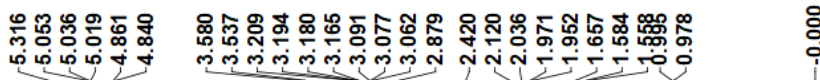

$\underbrace{M e}_{M e}$

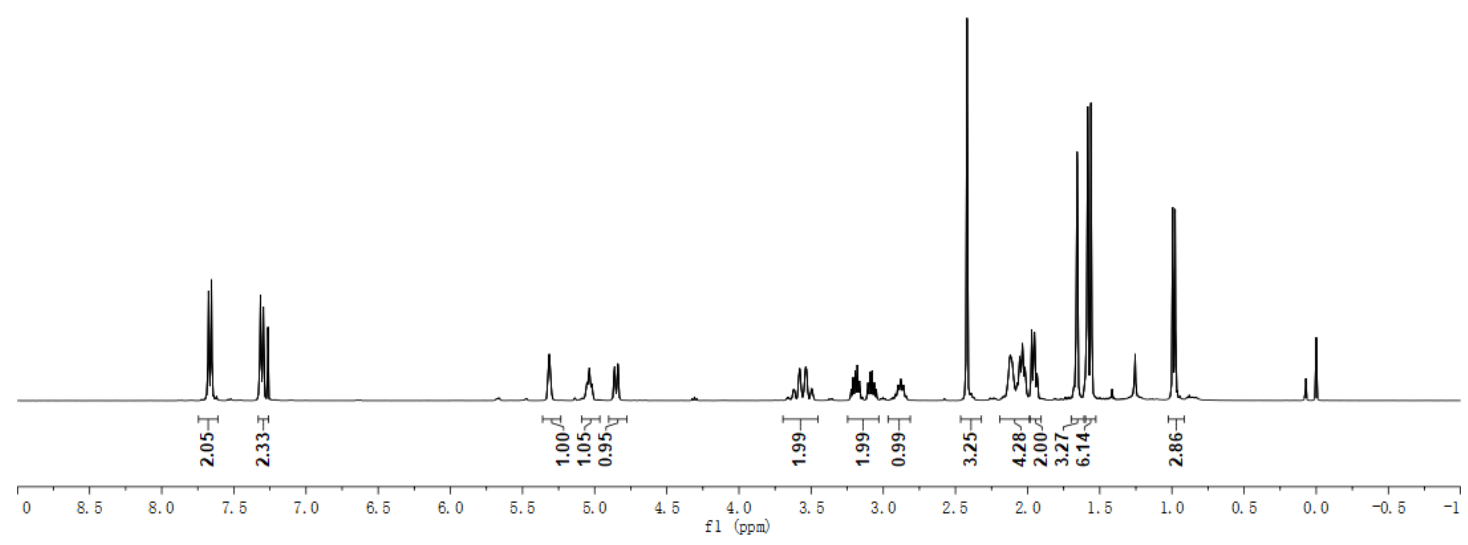

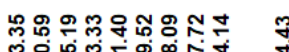

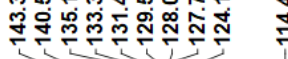

뚜윰ำ

אरำ

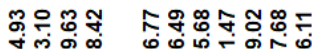

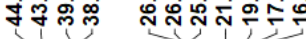

$\overbrace{M e}^{M e}$

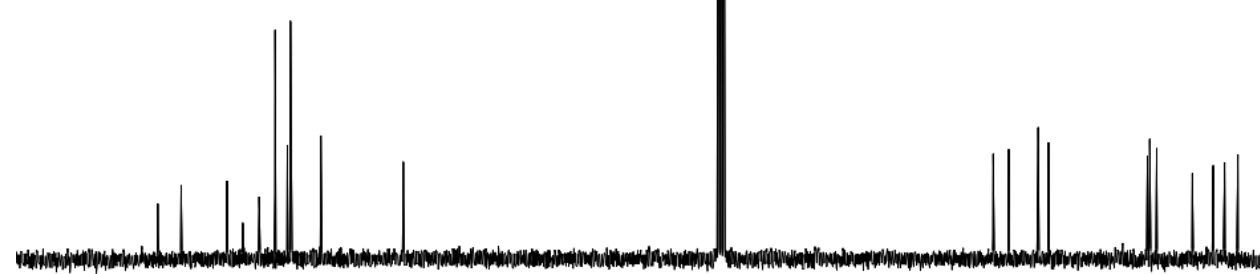

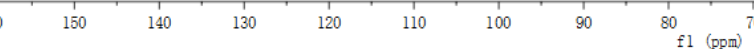


3ag; ${ }^{1} \mathrm{H}$ NMR (400MHz, $\left.\mathrm{CDCl}_{3}\right) ;{ }^{13} \mathrm{C}$ NMR $\left(100 \mathrm{MHz}, \mathrm{CDCl}_{3}\right)$

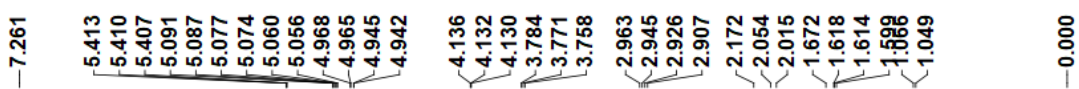

Me $\overbrace{\text { Me }}^{\mathrm{Me}}$

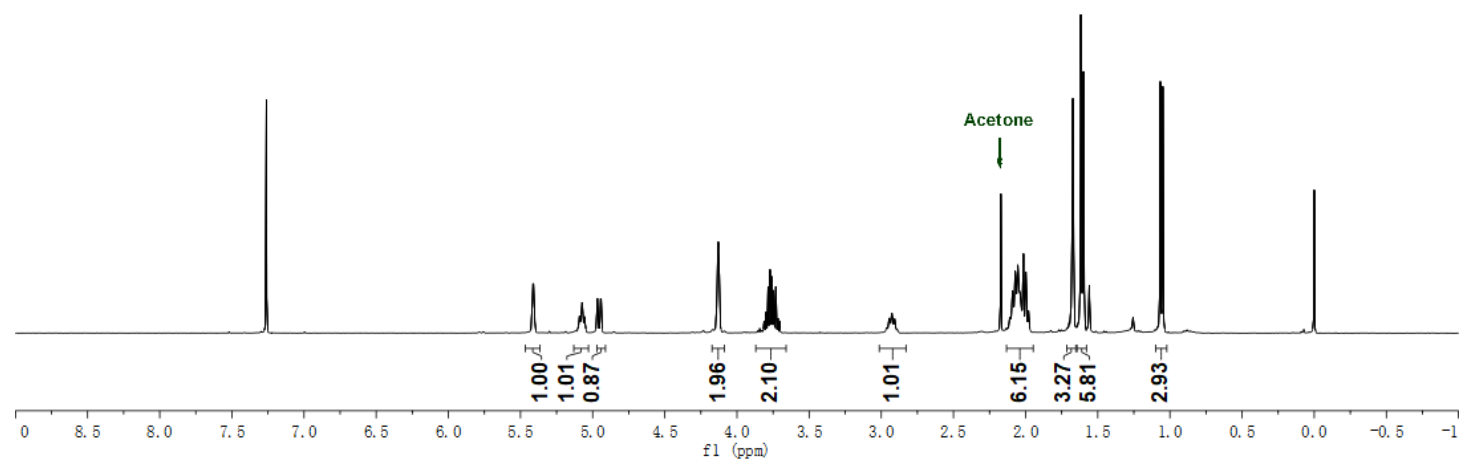

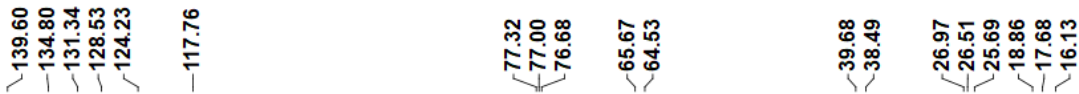
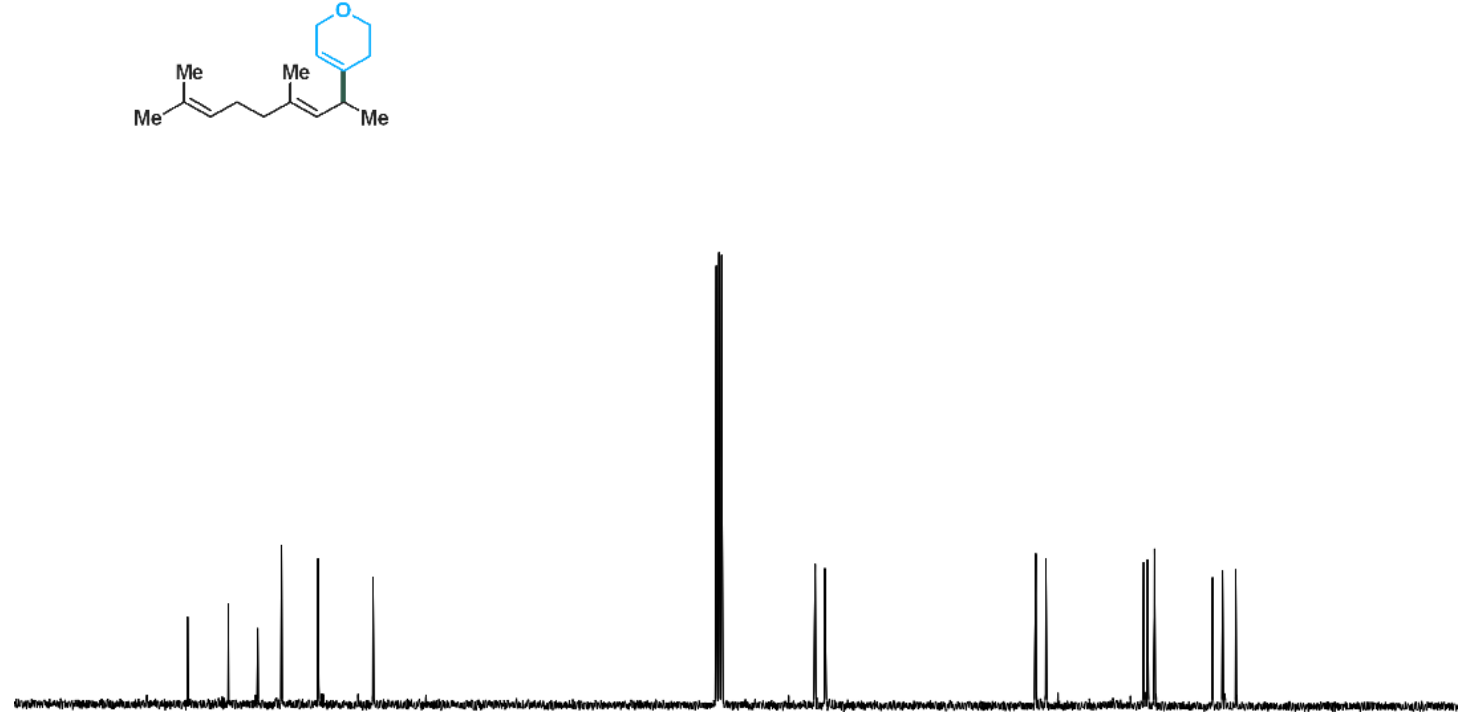

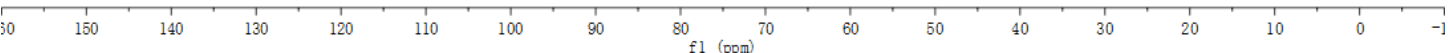


<smiles>CC(C)=CCC/C(C)=C/C(C)C1=CCSCC1</smiles>
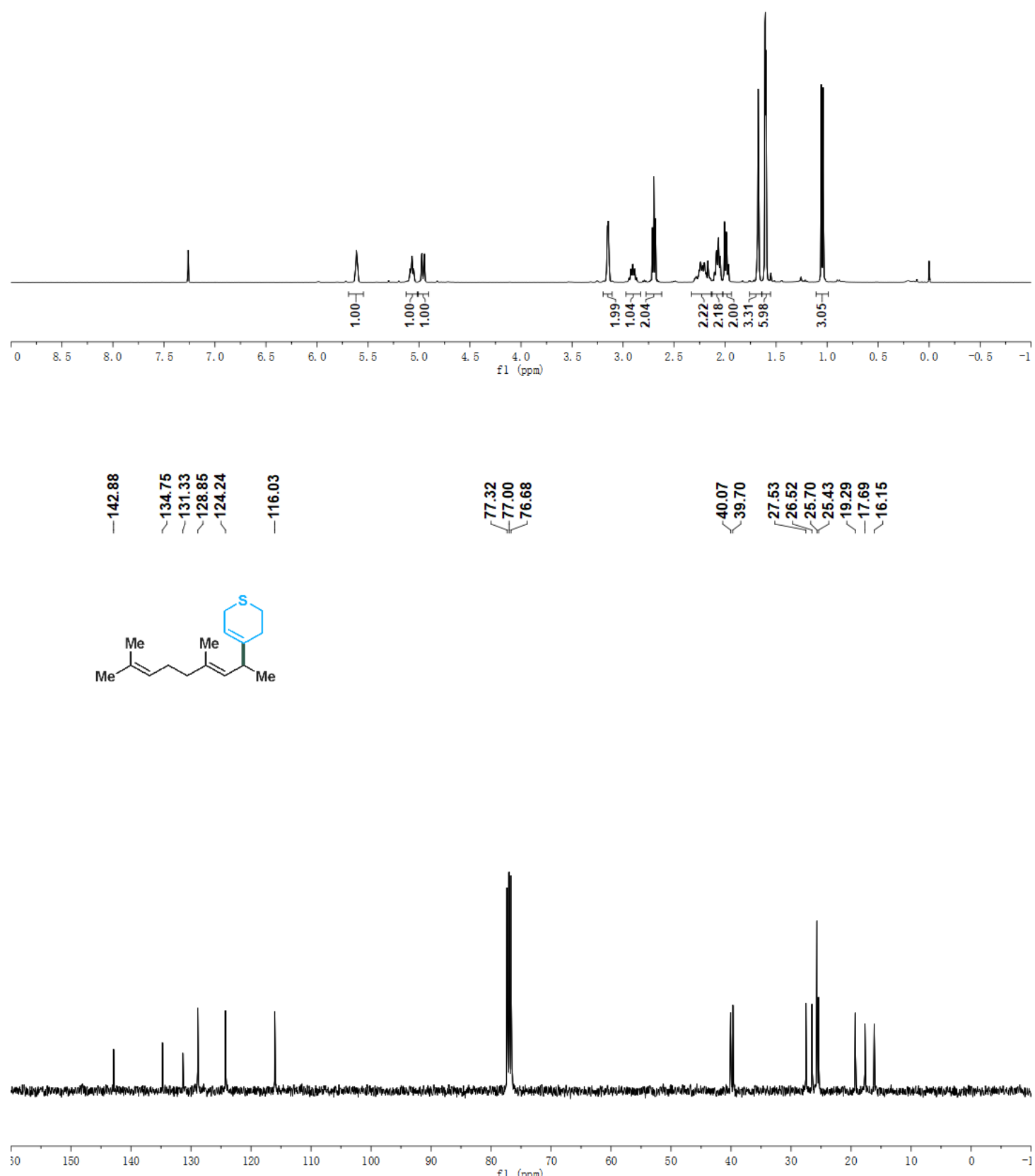
3ai; ${ }^{1} \mathrm{H}$ NMR (600MHz, $\left.\mathrm{CDCl}_{3}\right) ;{ }^{13} \mathrm{C} \mathrm{NMR}\left(100 \mathrm{MHz}, \mathrm{CDCl}_{3}\right)$

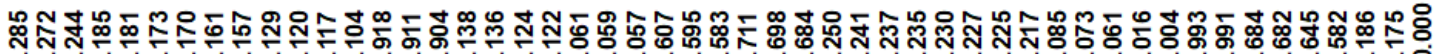

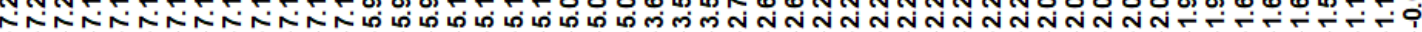<smiles>C=C(C=C(N)CCC=C(C)C)C1=C2C=CC=CC2CCC1</smiles>

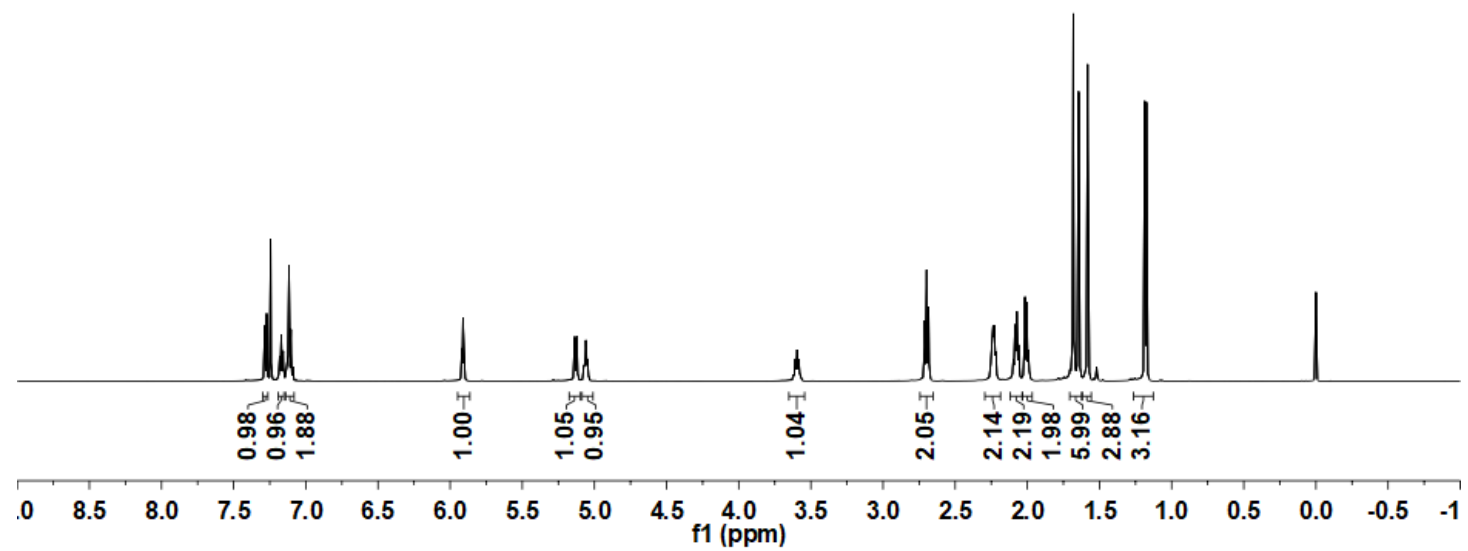

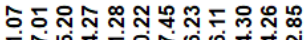

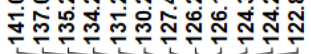

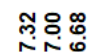

余管

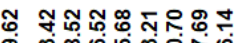

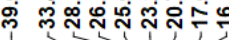
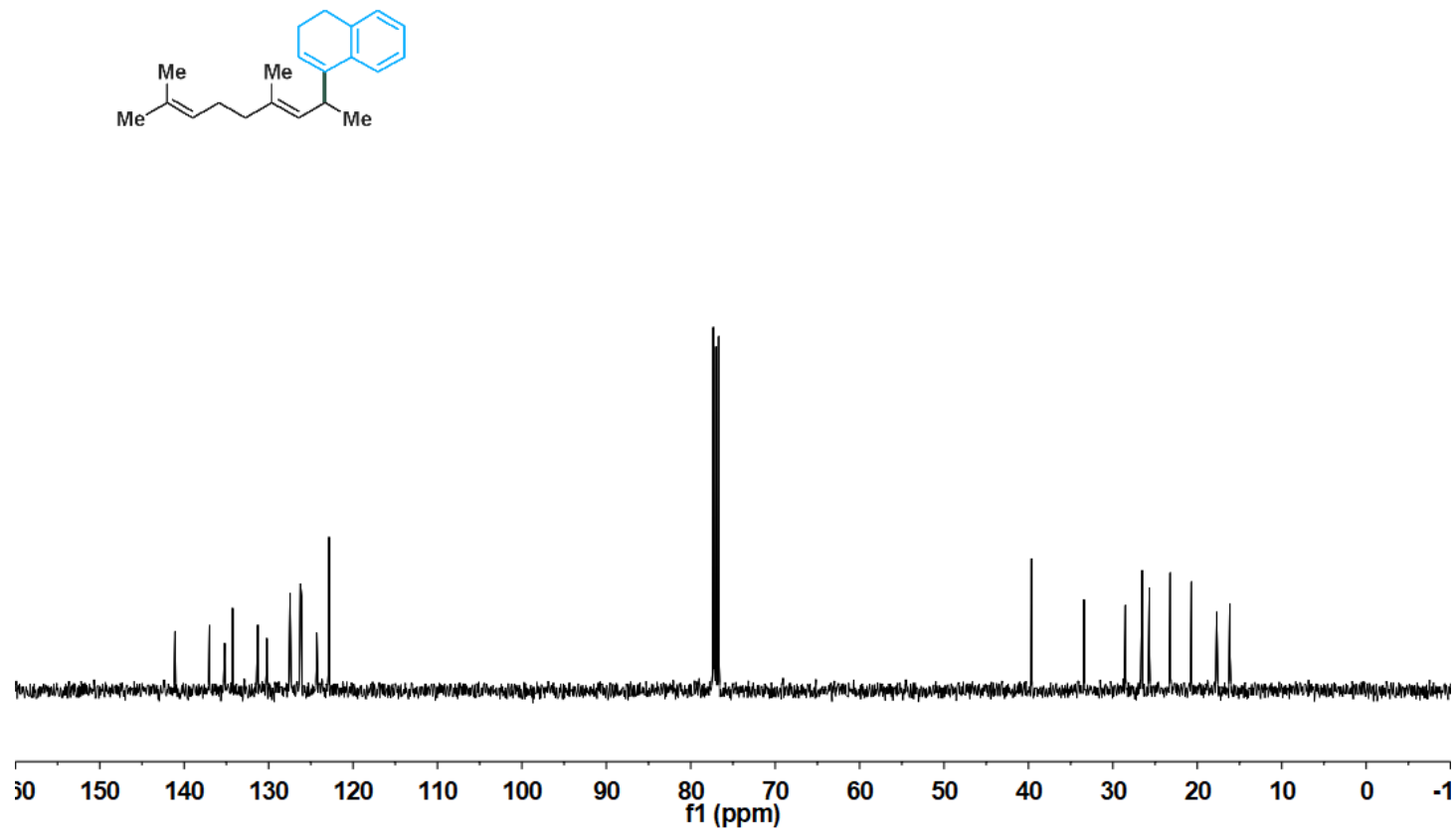
3aj; ${ }^{1} \mathrm{H}$ NMR (400MHz, $\left.\mathrm{CDCl}_{3}\right) ;{ }^{13} \mathrm{C}$ NMR (100 MHz, CDCl $)$

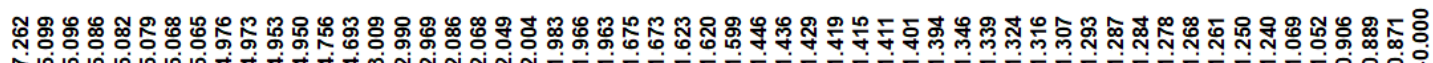

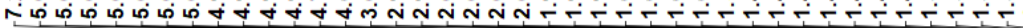

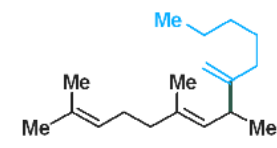

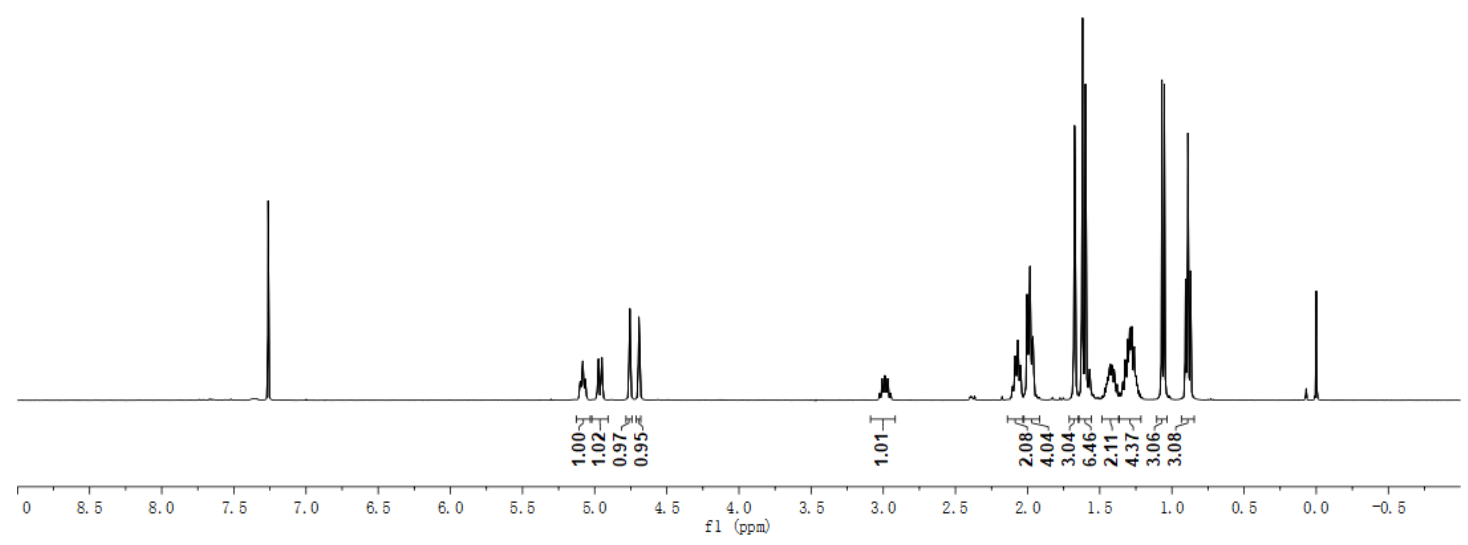

\begin{tabular}{|c|c|c|c|}
\hline 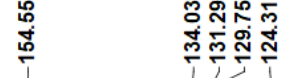 & $\begin{array}{l}\stackrel{\infty}{\circ} \\
\stackrel{\circ}{\circ}\end{array}$ & 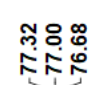 & 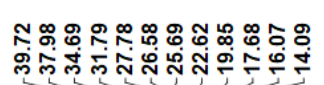 \\
\hline
\end{tabular}

Me Me

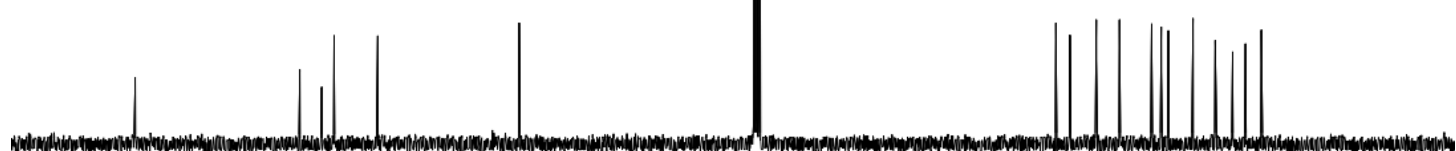

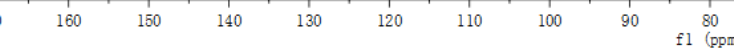


3ak; ${ }^{1} \mathrm{H}$ NMR (600MHz, $\left.\mathrm{CDCl}_{3}\right) ;{ }^{13} \mathrm{C}$ NMR $\left(150 \mathrm{MHz}, \mathrm{CDCl}_{3}\right)$

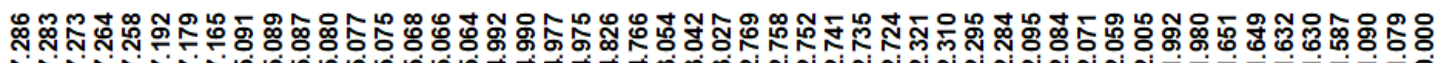

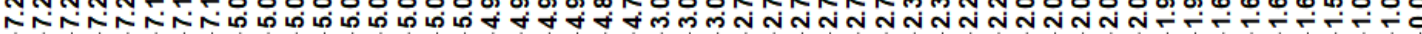

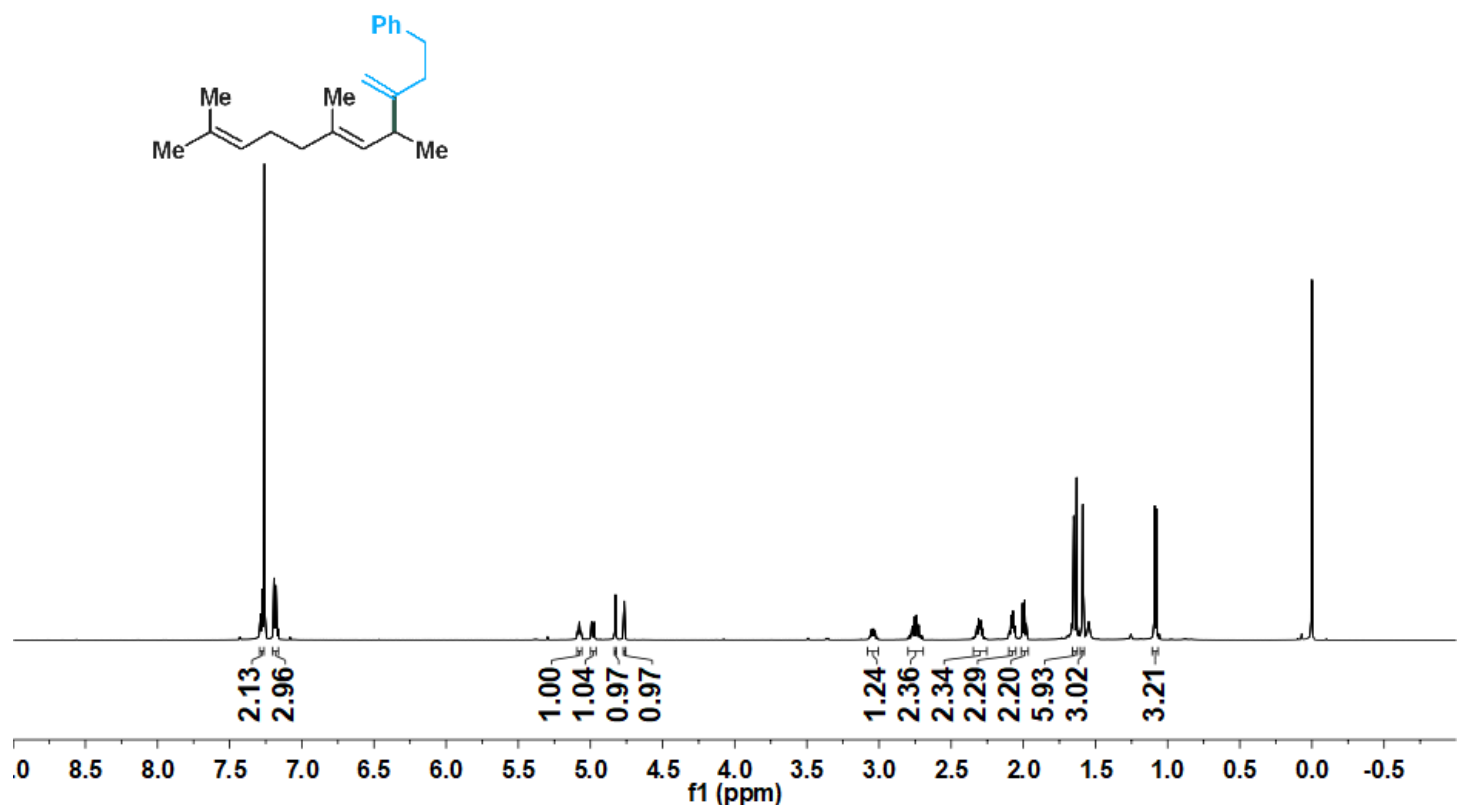

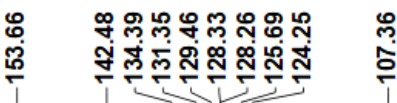

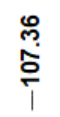

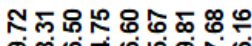

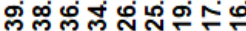
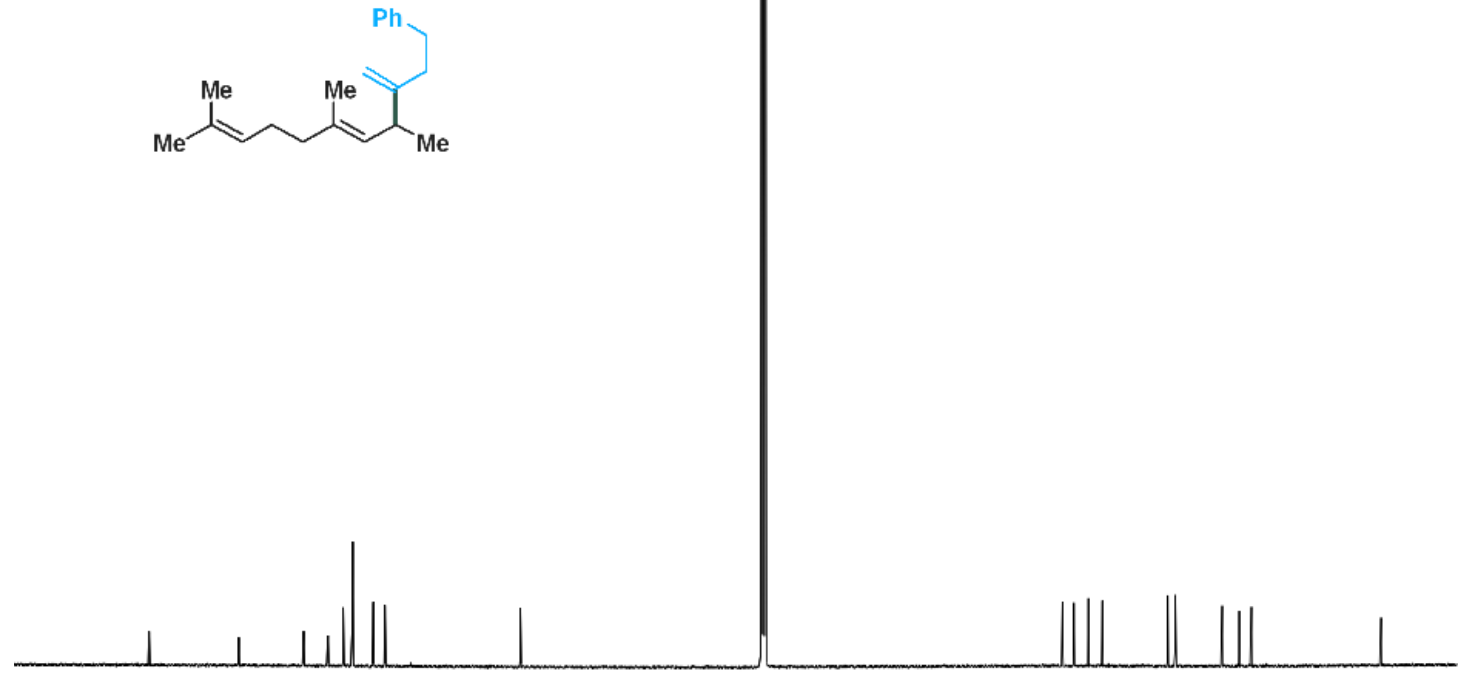

$\begin{array}{llllllllll}70 & 160 & 150 & 140 & 130 & 120 & 110 & 100 & 90 & 80 \\ \mathrm{f} 1(\mathrm{ppm})\end{array}$ 

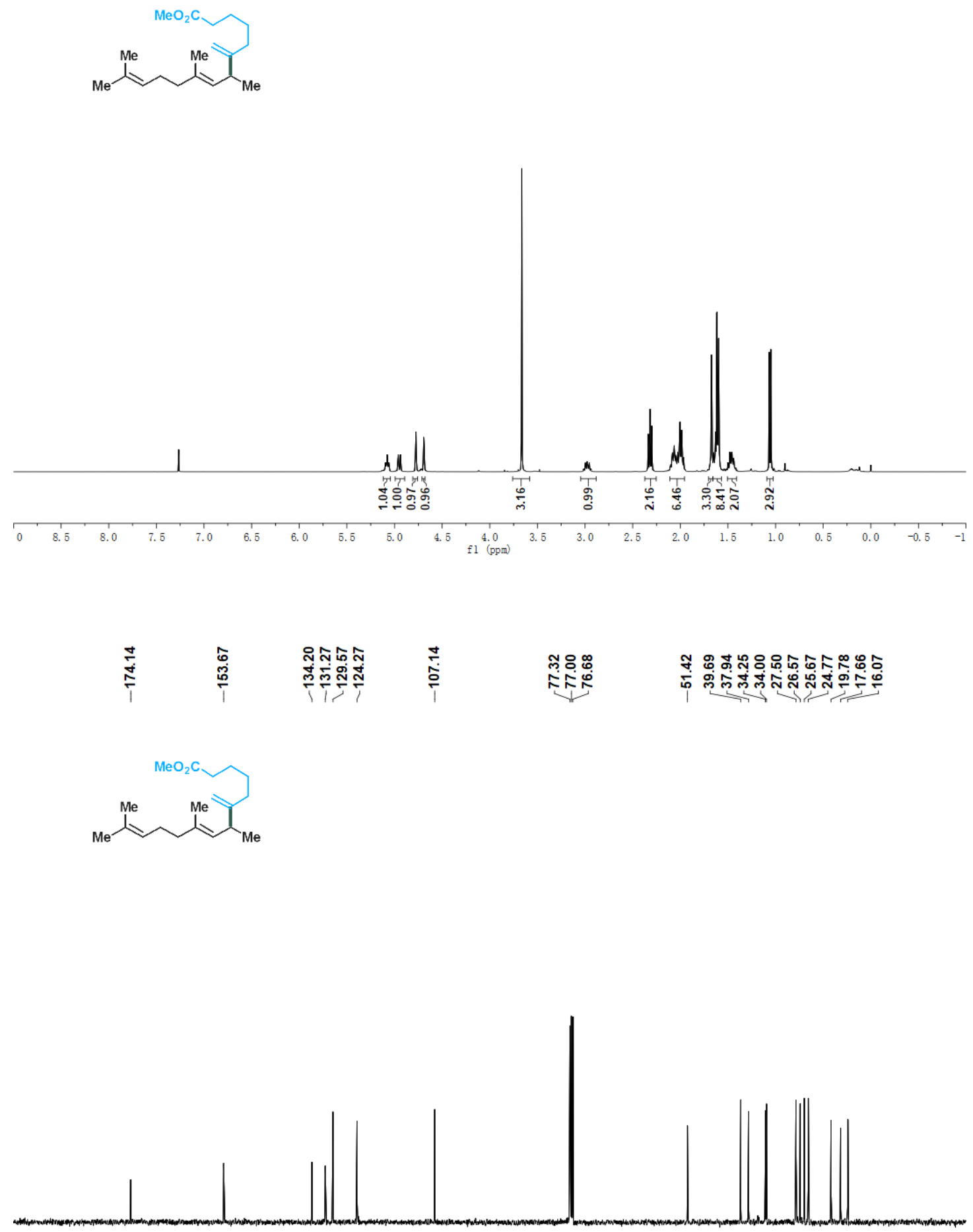

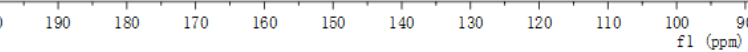


3am; ${ }^{1} \mathrm{H}$ NMR (400MHz, $\left.\mathrm{CDCl}_{3}\right) ;{ }^{13} \mathrm{C}$ NMR (100 MHz, $\left.\mathrm{CDCl}_{3}\right)$

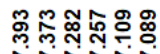

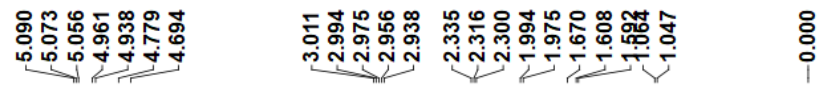

me

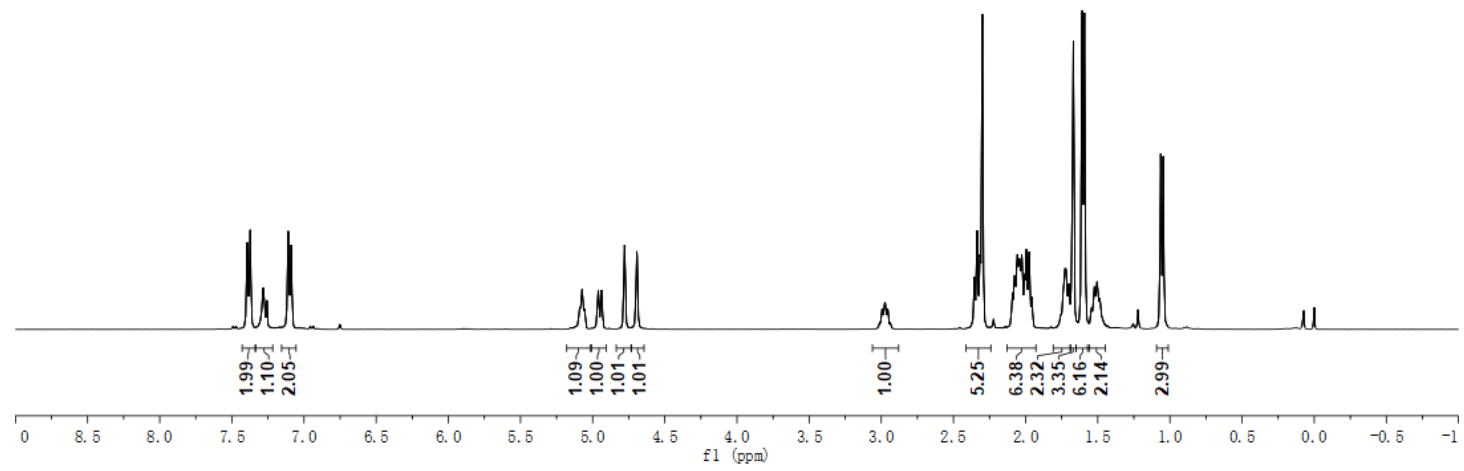

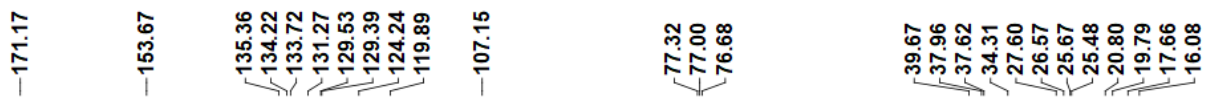
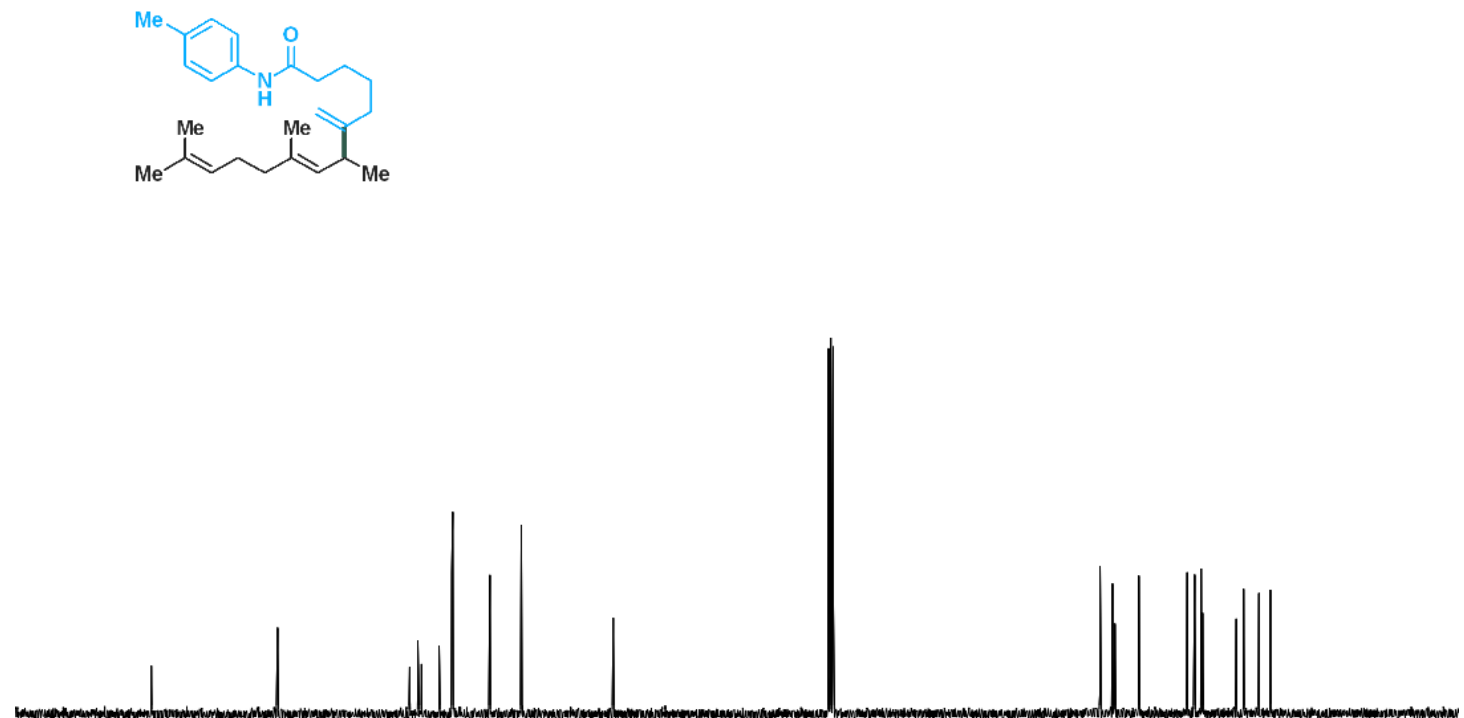

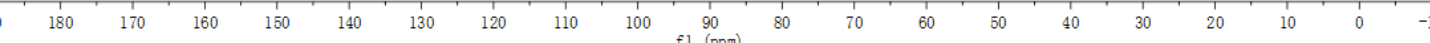



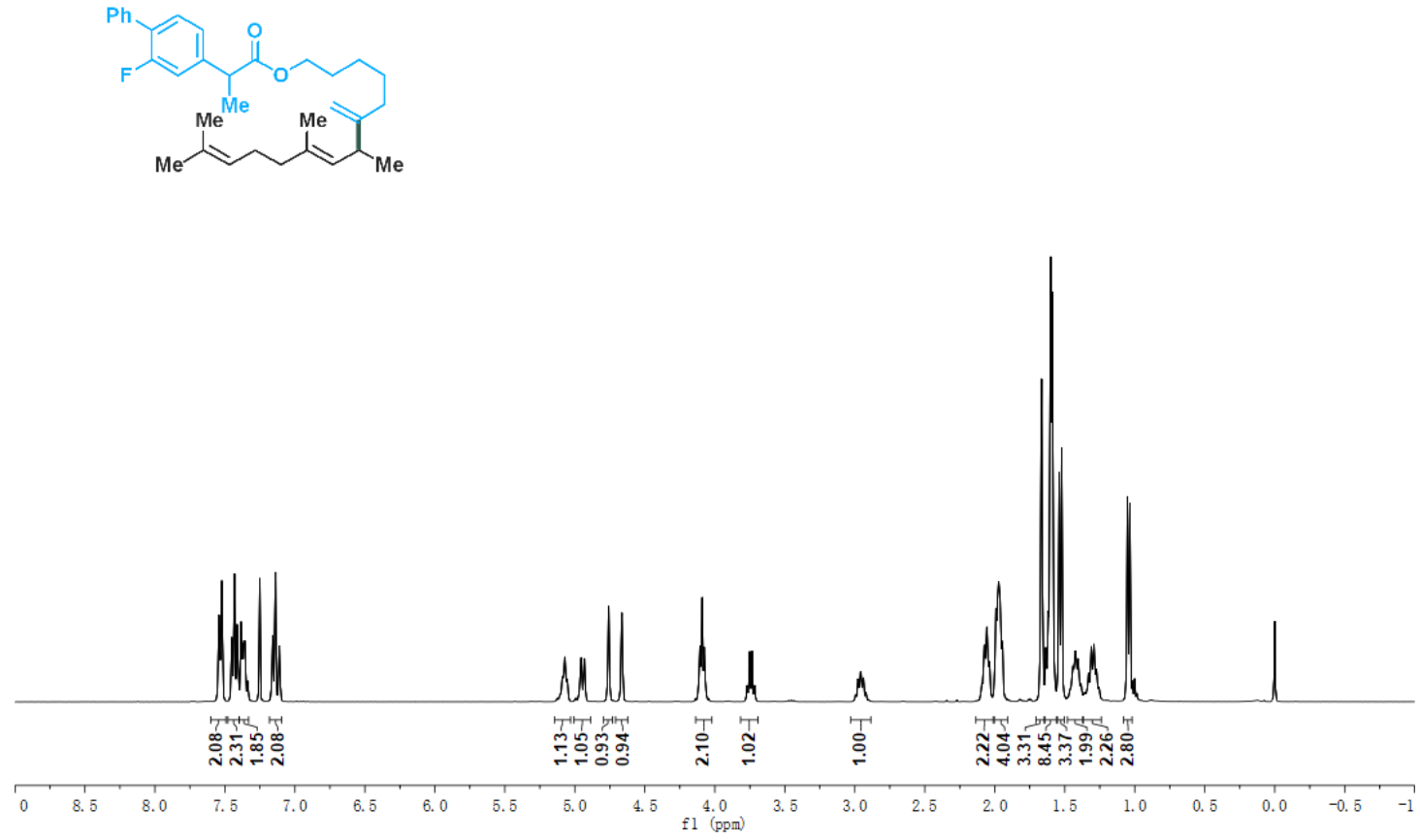

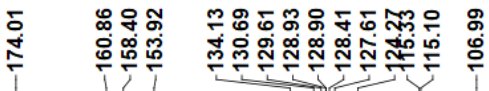

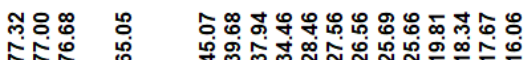

称
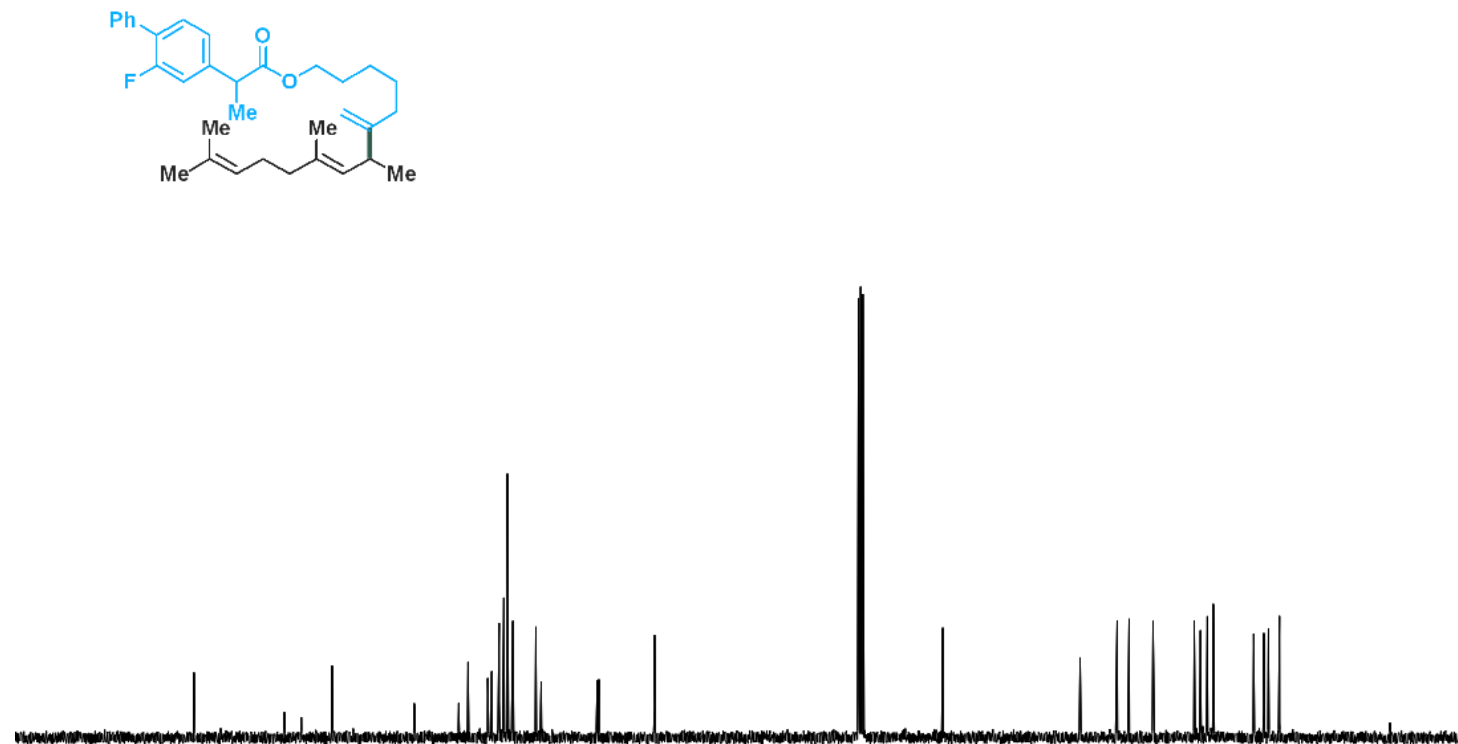

$\begin{array}{llllllllll}190 & 180 & 170 & 160 & 150 & 140 & 130 & 120 & 110 & 100 \\ \mathrm{f} 1(\mathrm{ppm})\end{array}$ 
3an; ${ }^{19}$ F NMR (376 $\mathrm{MHz}, \mathrm{CDCl}_{3}$ )

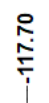
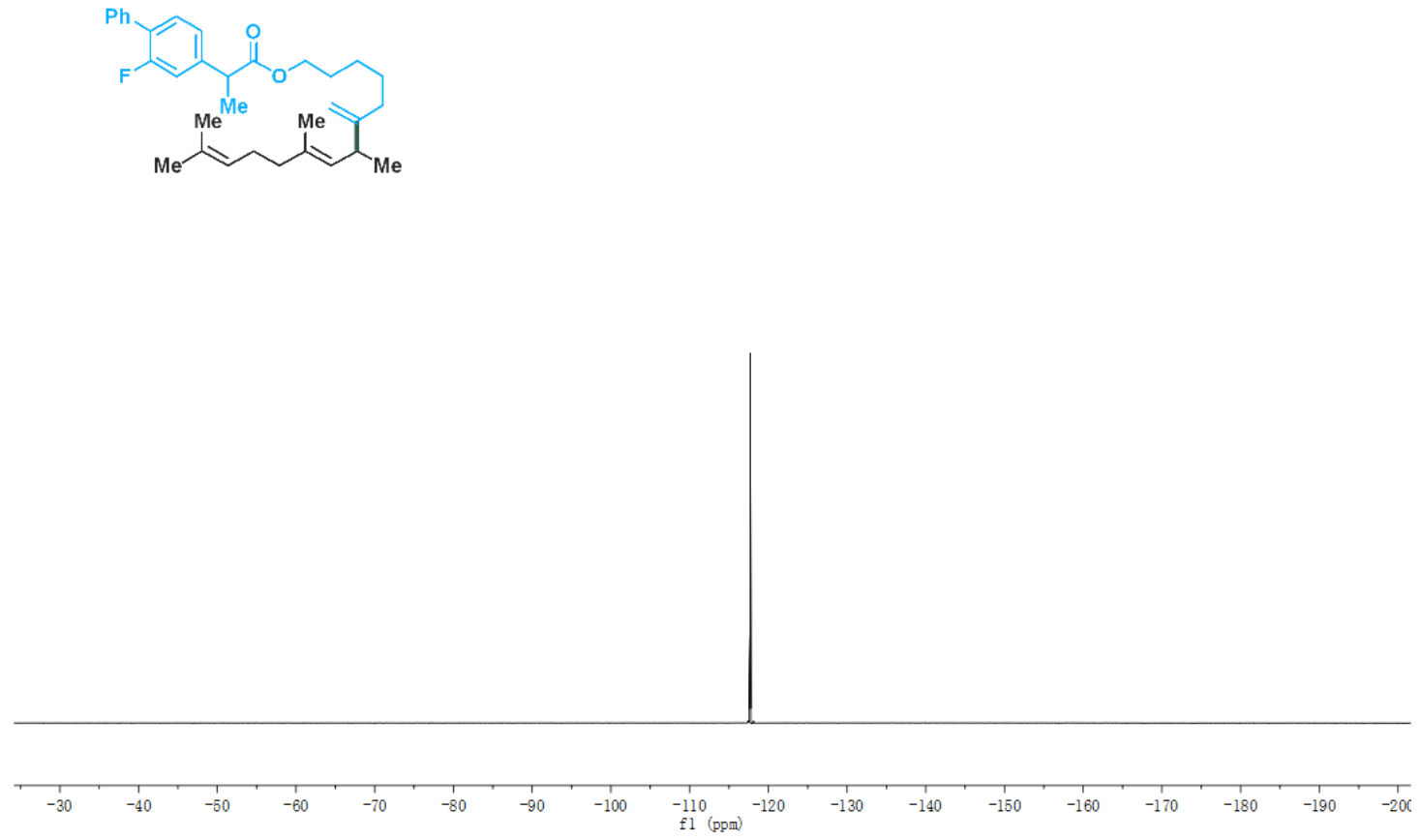
3ao; ${ }^{1} \mathrm{H}$ NMR (400MHz, $\left.\mathrm{CDCl}_{3}\right) ;{ }^{13} \mathrm{C}$ NMR $\left(100 \mathrm{MHz}, \mathrm{CDCl}_{3}\right)$

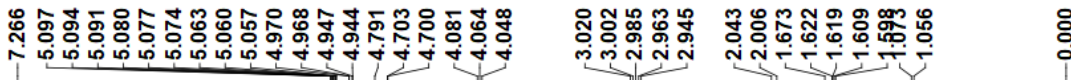 \\ $\stackrel{\circ}{i}$}

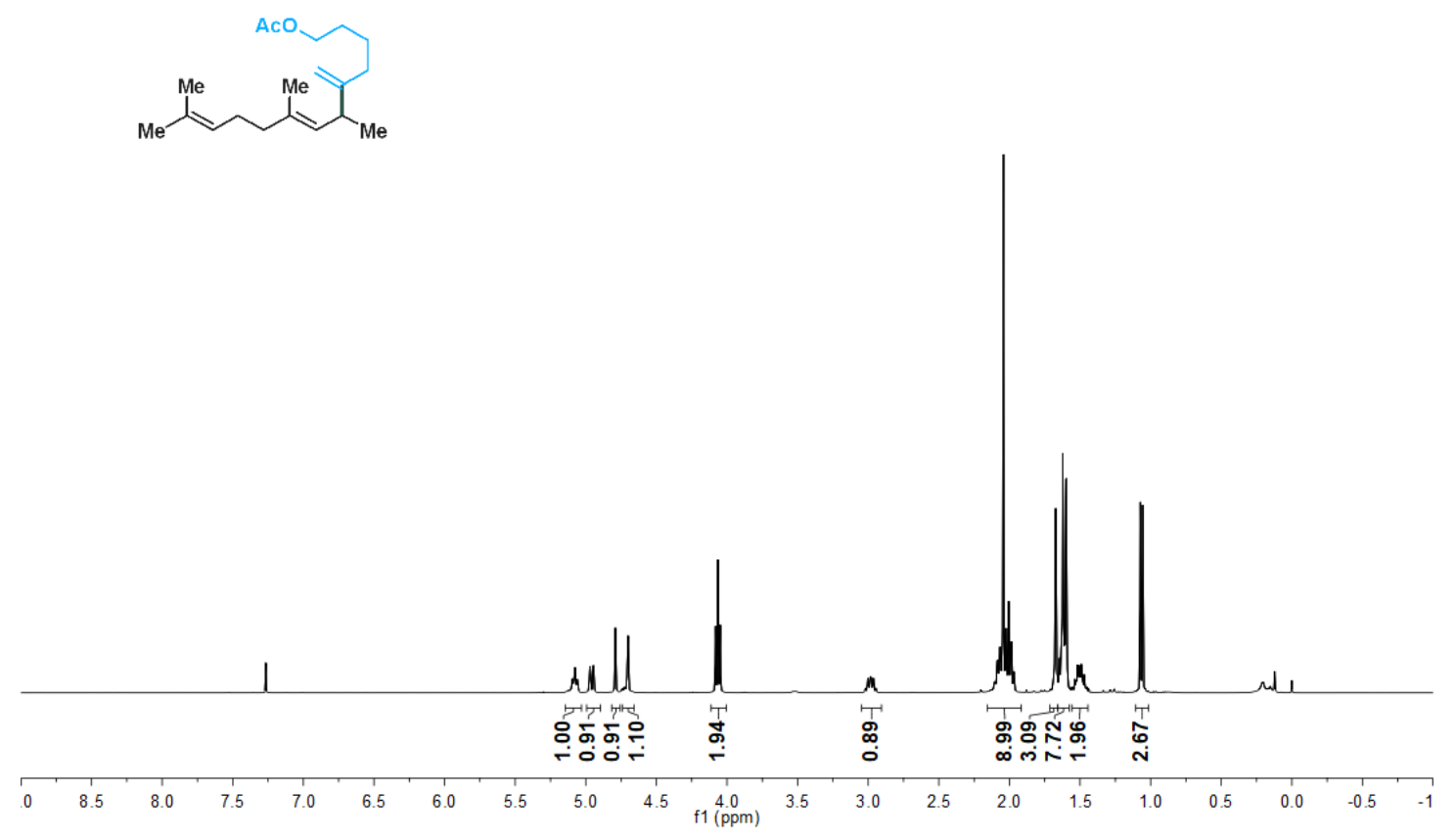

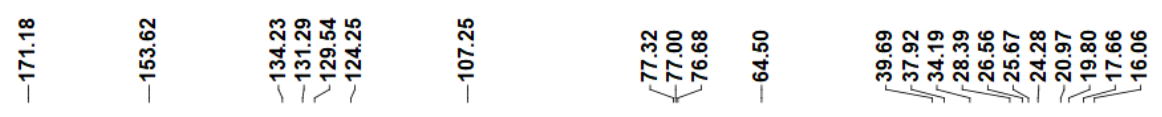<smiles>C=C(CCC=C(C)C)C(=C)CCCCCCC(C)=O</smiles>

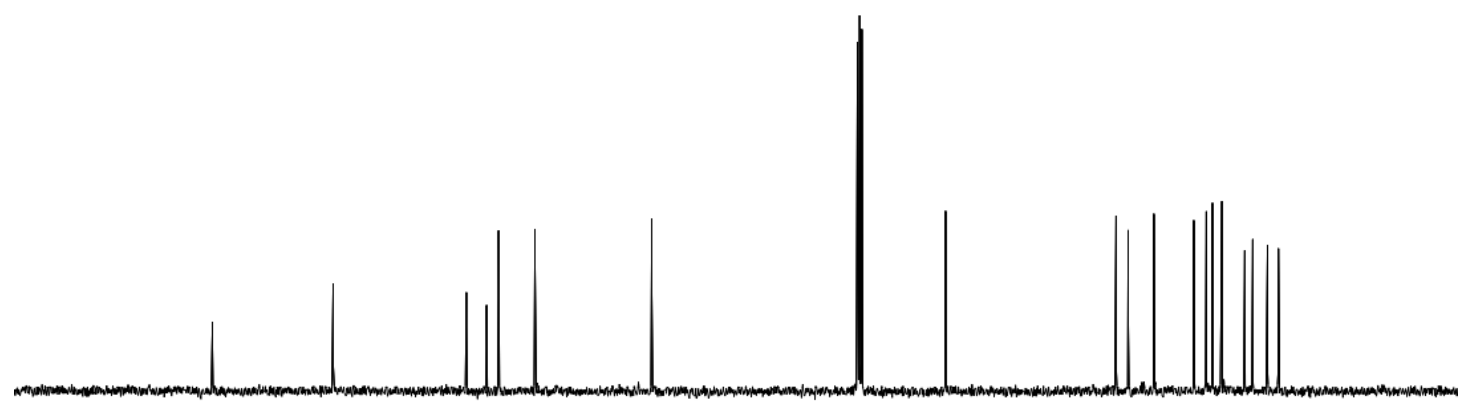

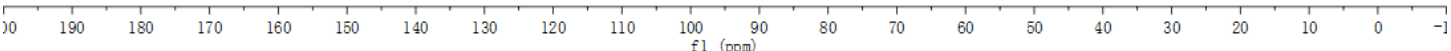


3ap; ${ }^{1} \mathrm{H}$ NMR (400MHz, $\left.\mathrm{CDCl}_{3}\right) ;{ }^{13} \mathrm{C}$ NMR (100 $\left.\mathrm{MHz}, \mathrm{CDCl}_{3}\right)$

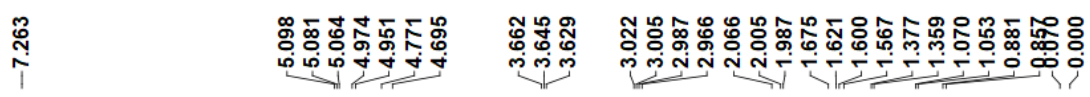

Me Me

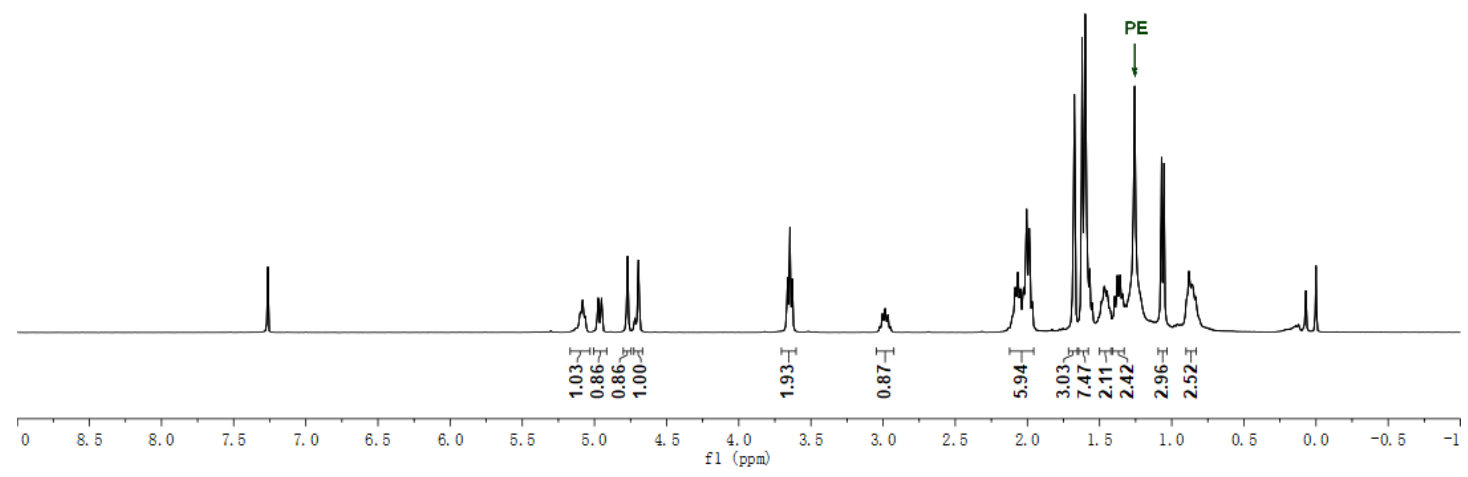

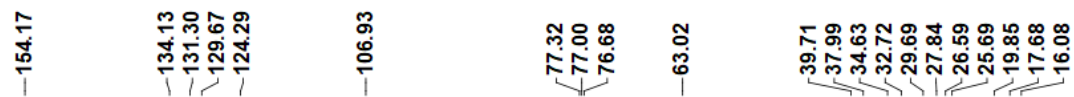
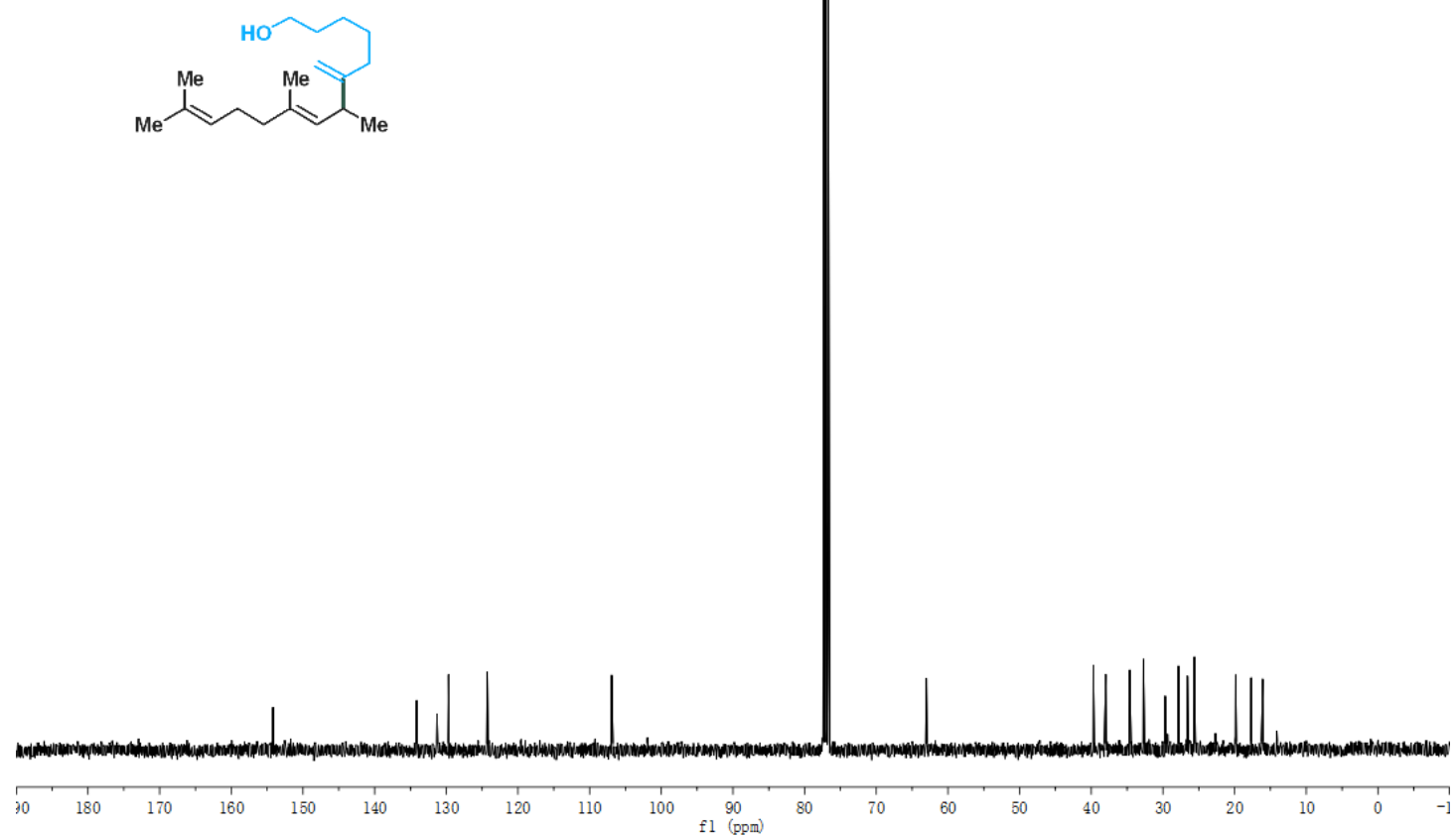
6; ${ }^{1} \mathrm{H}$ NMR (400MHz, $\left.\mathrm{CDCl}_{3}\right) ;{ }^{13} \mathrm{C}$ NMR (100 $\left.\mathrm{MHz}, \mathrm{CDCl}_{3}\right)$

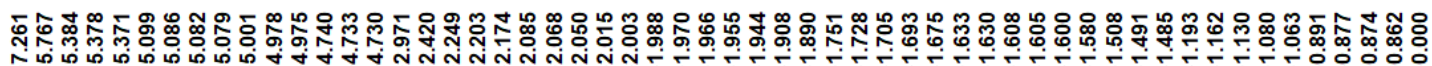
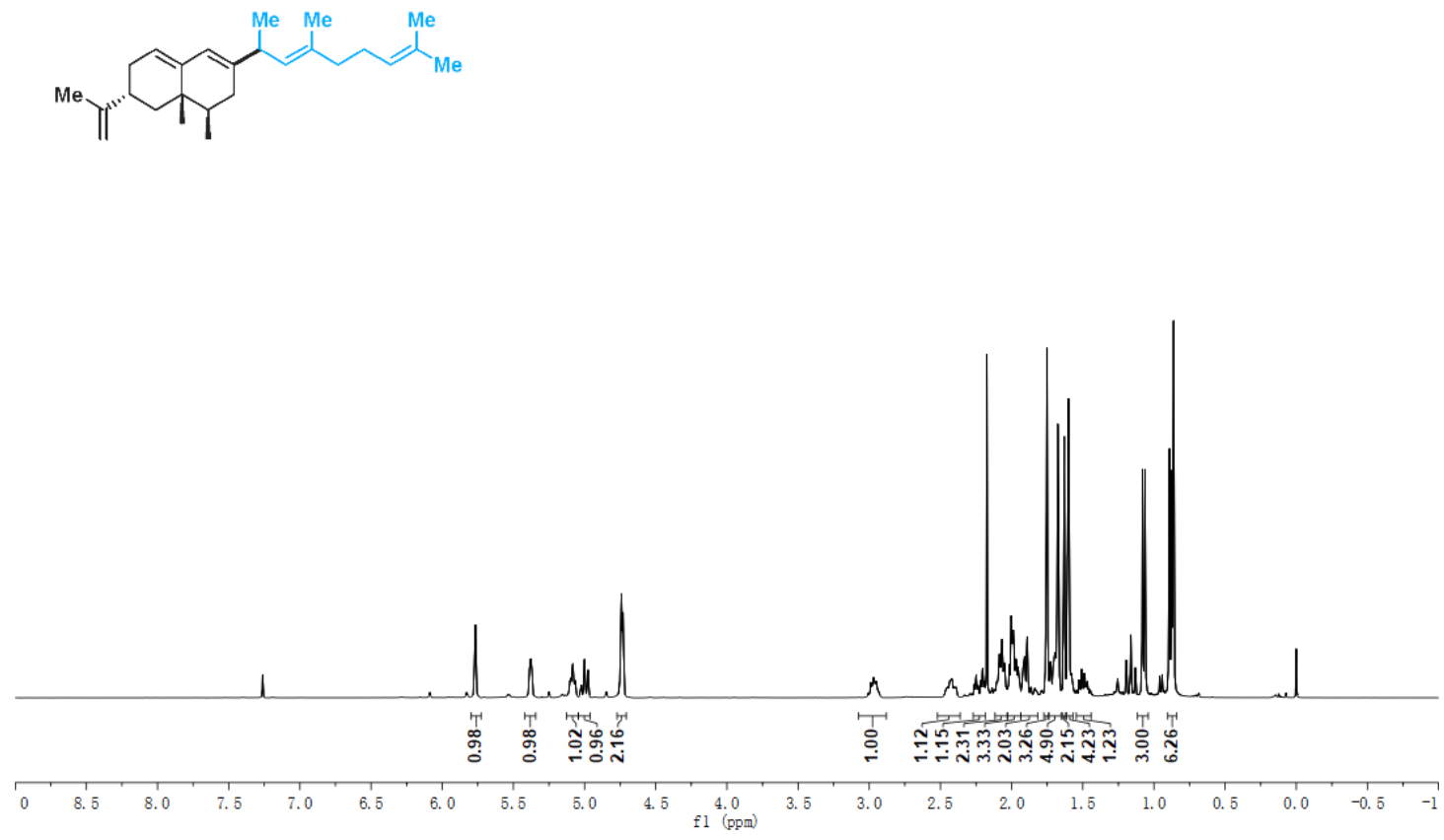

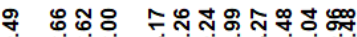

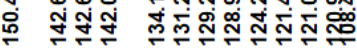

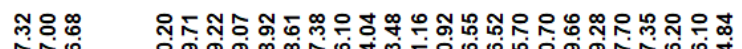

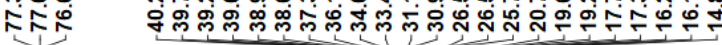

Me

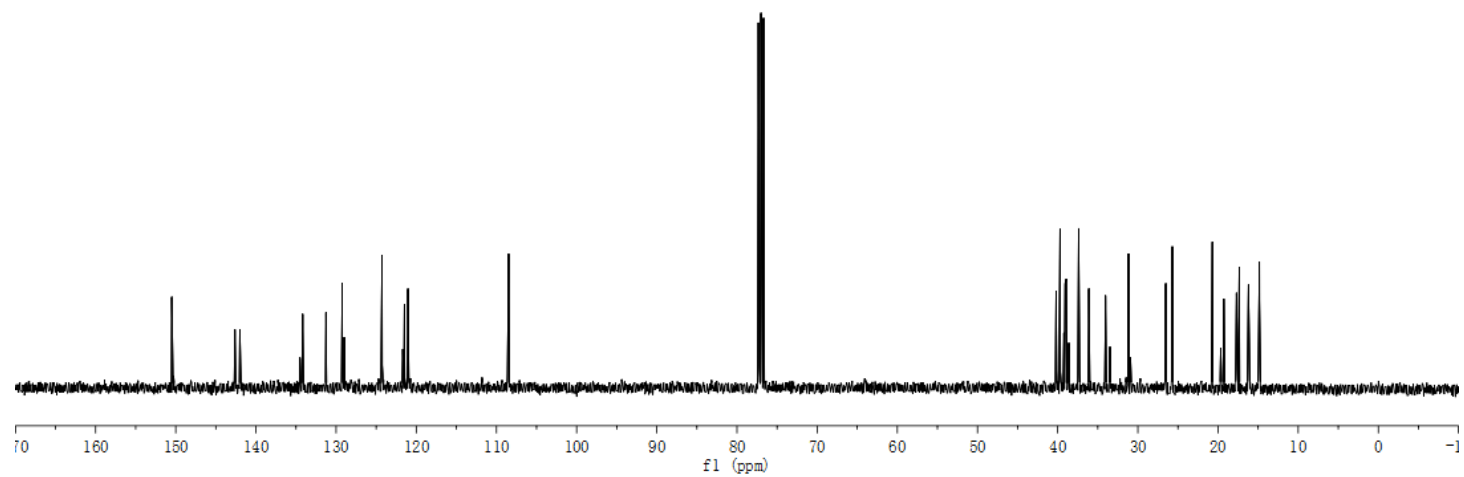

$\mathrm{S} 135$ 
8; ${ }^{1} \mathrm{H}$ NMR (400MHz, $\left.\mathrm{CDCl}_{3}\right) ;{ }^{13} \mathrm{C}$ NMR (100 $\left.\mathrm{MHz}, \mathrm{CDCl}_{3}\right)$

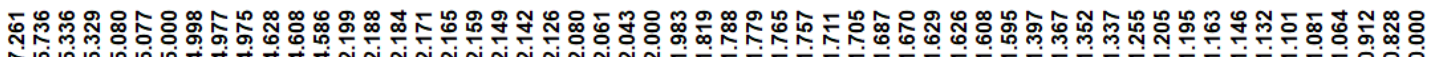

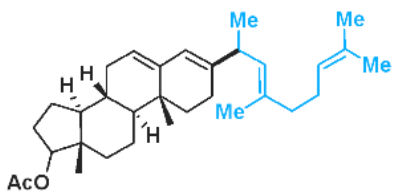

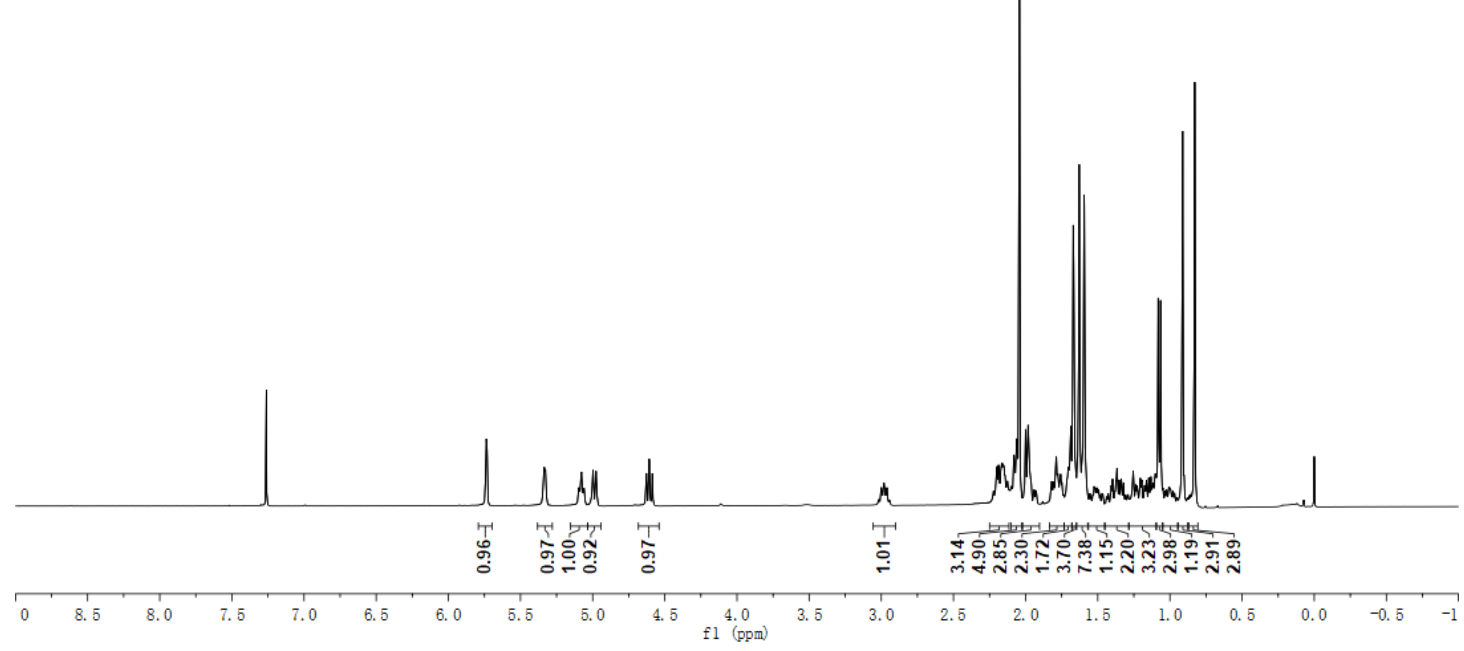

要

๓

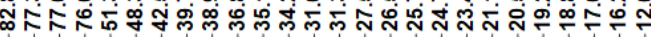

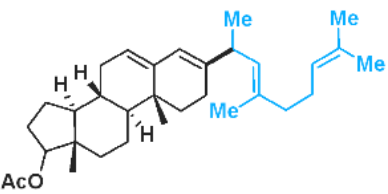
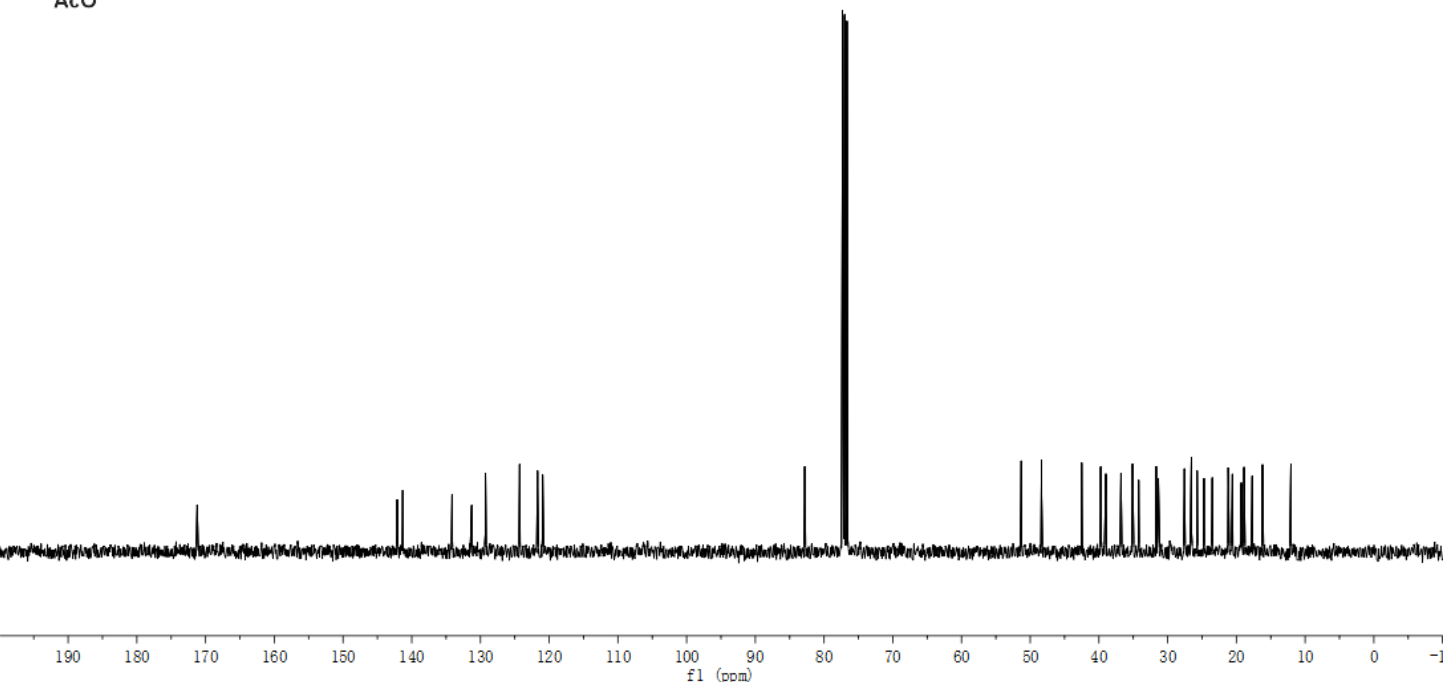

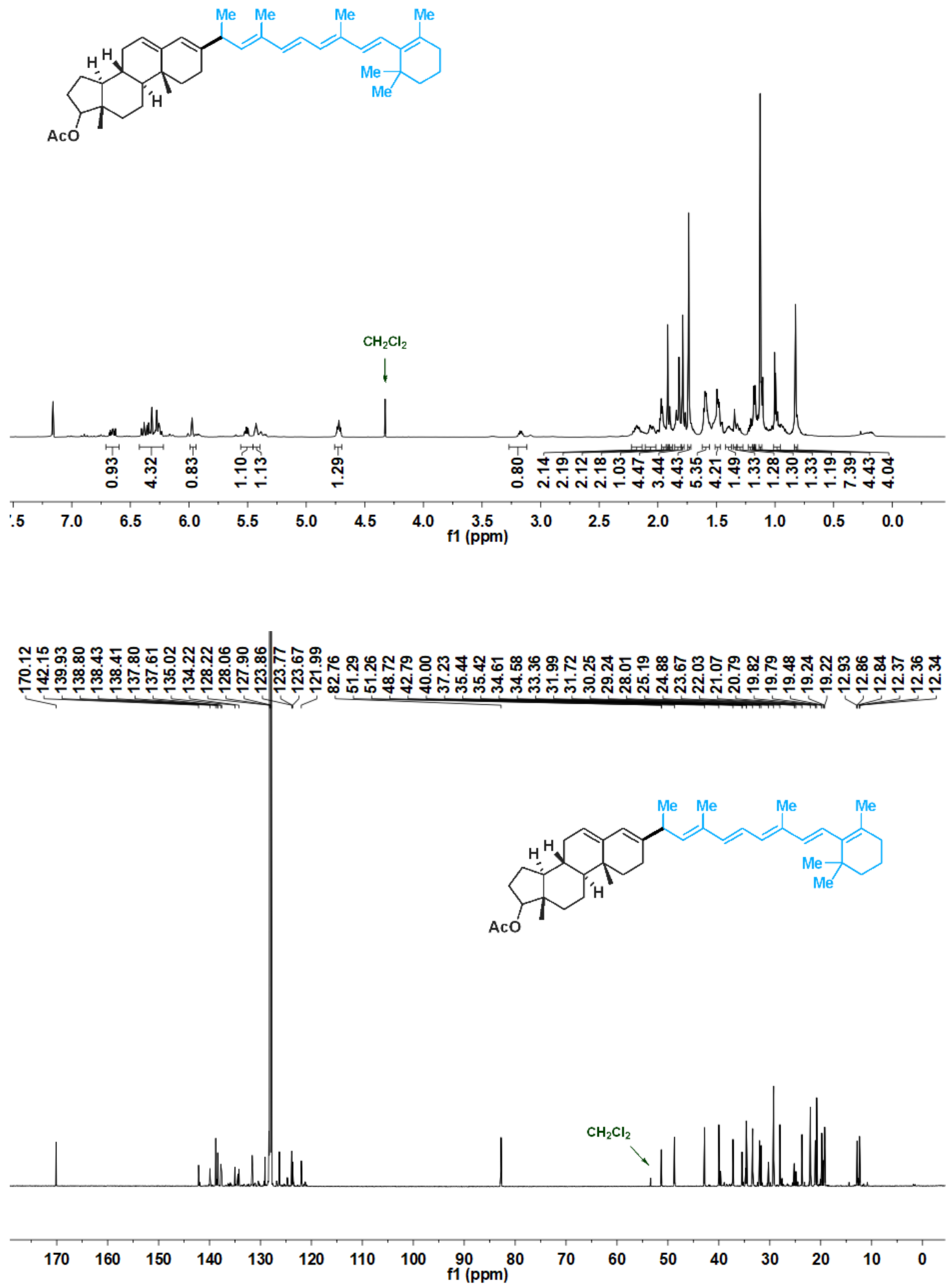
15; ${ }^{1} \mathrm{H}$ NMR (400MHz, $\left.\mathrm{CDCl}_{3}\right) ;{ }^{13} \mathrm{C}$ NMR (100 MHz, $\left.\mathrm{CDCl}_{3}\right)$

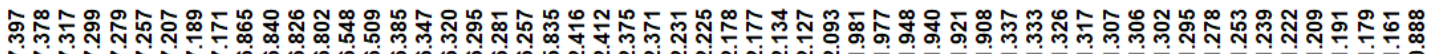
Ninvinti.

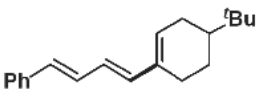

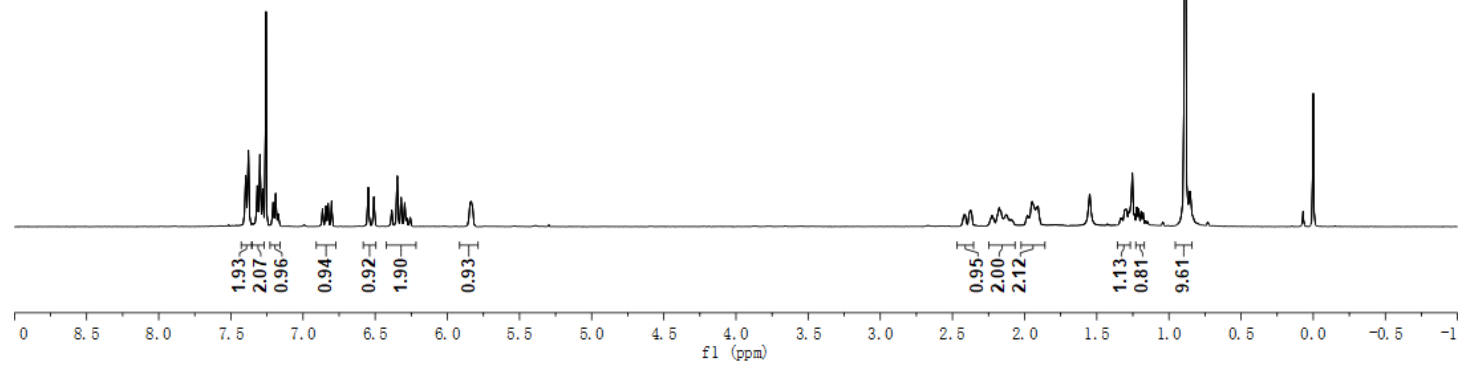

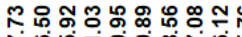

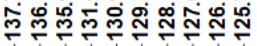

$$
\text { 象患: }
$$
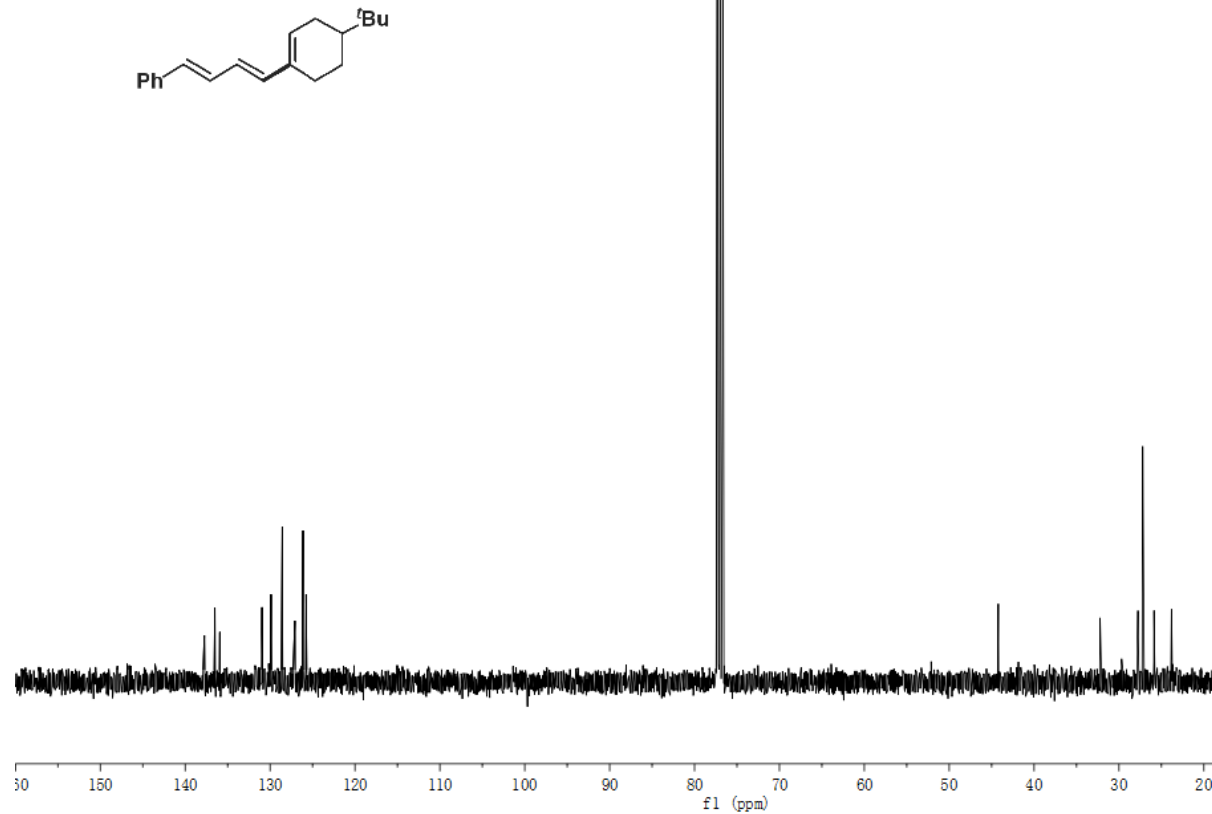
3ar; ${ }^{1} \mathrm{H}$ NMR (400MHz, $\left.\mathrm{CDCl}_{3}\right) ;{ }^{13} \mathrm{C}$ NMR (100 $\left.\mathrm{MHz}, \mathrm{CDCl}_{3}\right)$

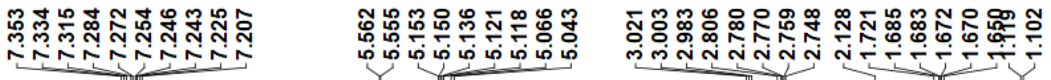
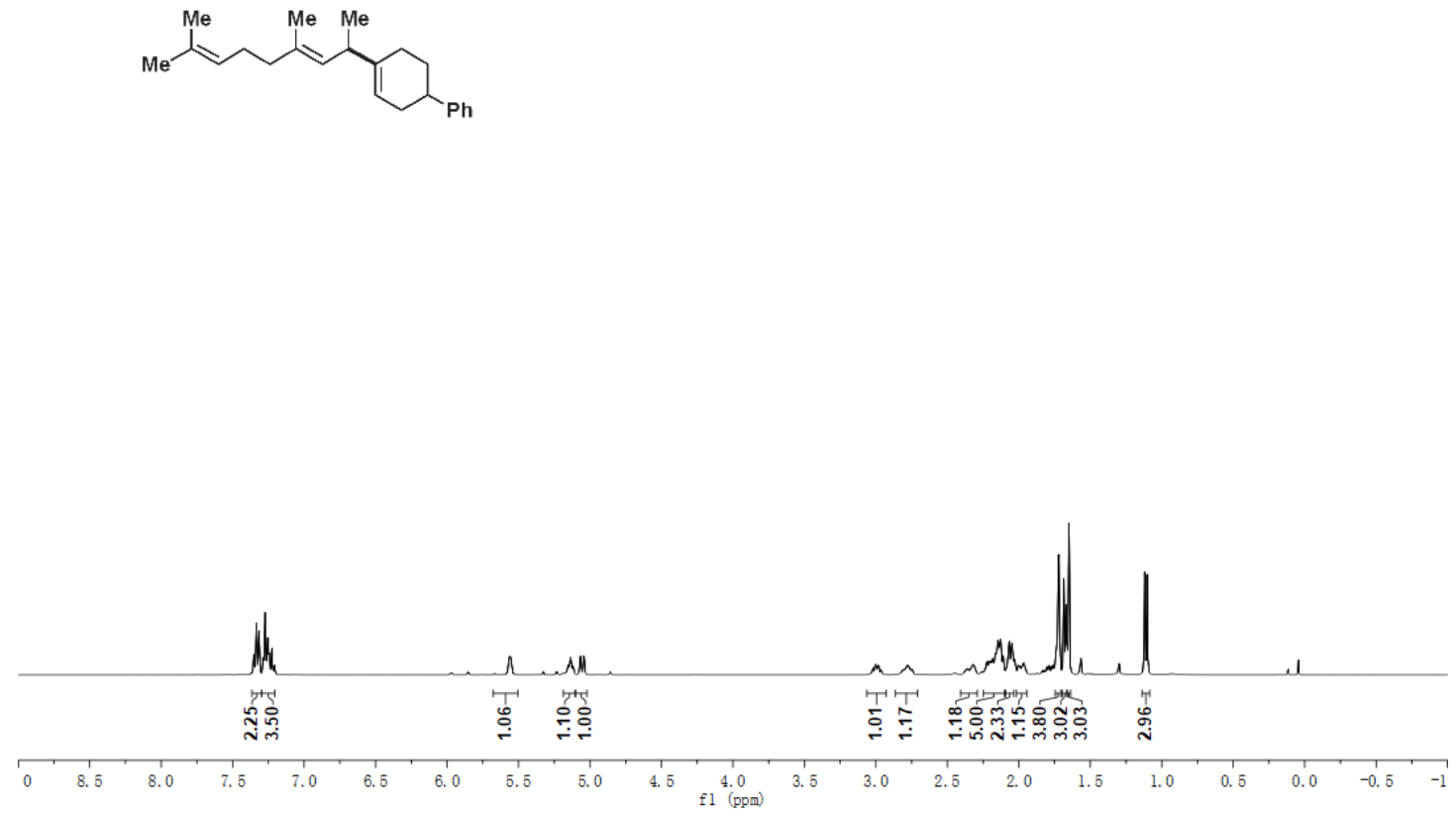

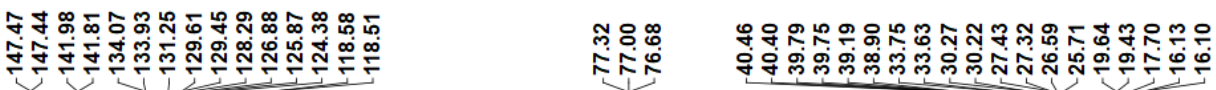

$\overbrace{\text { Ph }}^{\mathrm{Me}}$

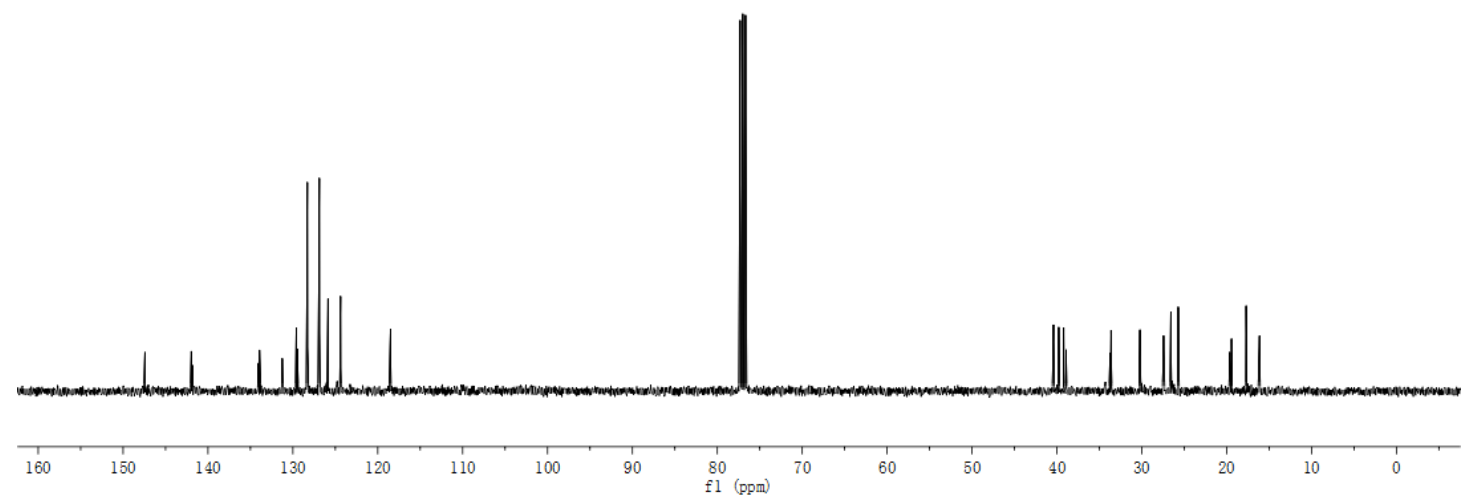


$E$-19; ${ }^{1} \mathrm{H}$ NMR (400MHz, $\left.\mathrm{CDCl}_{3}\right) ;{ }^{13} \mathrm{C}$ NMR (100 MHz, $\left.\mathrm{CDCl}_{3}\right)$

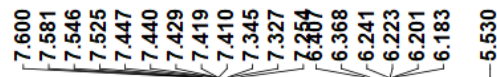

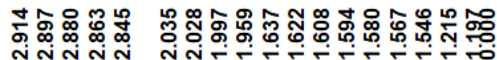

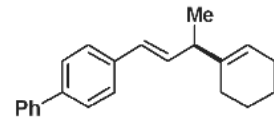

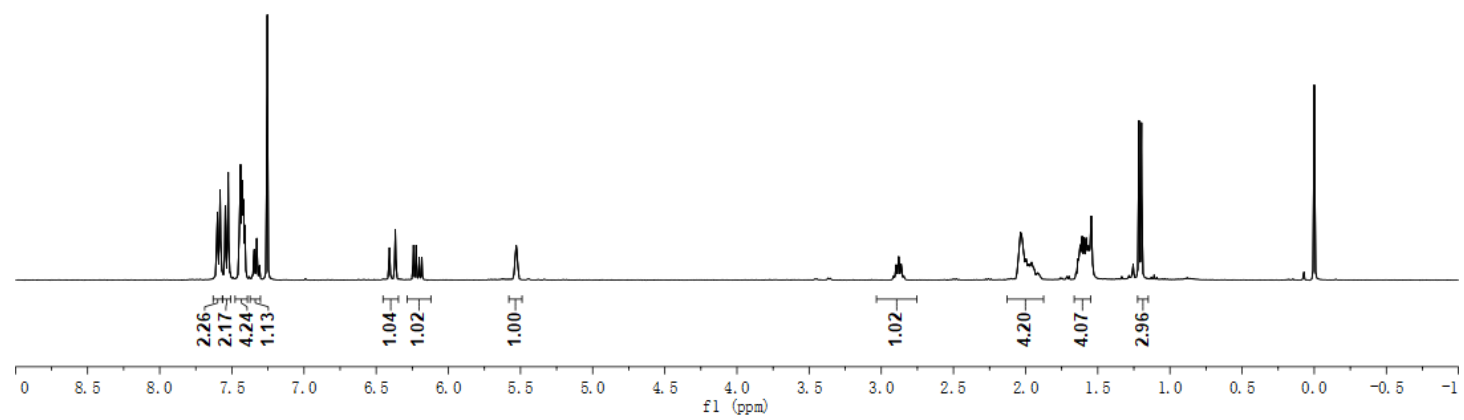

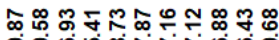

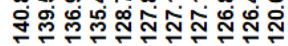
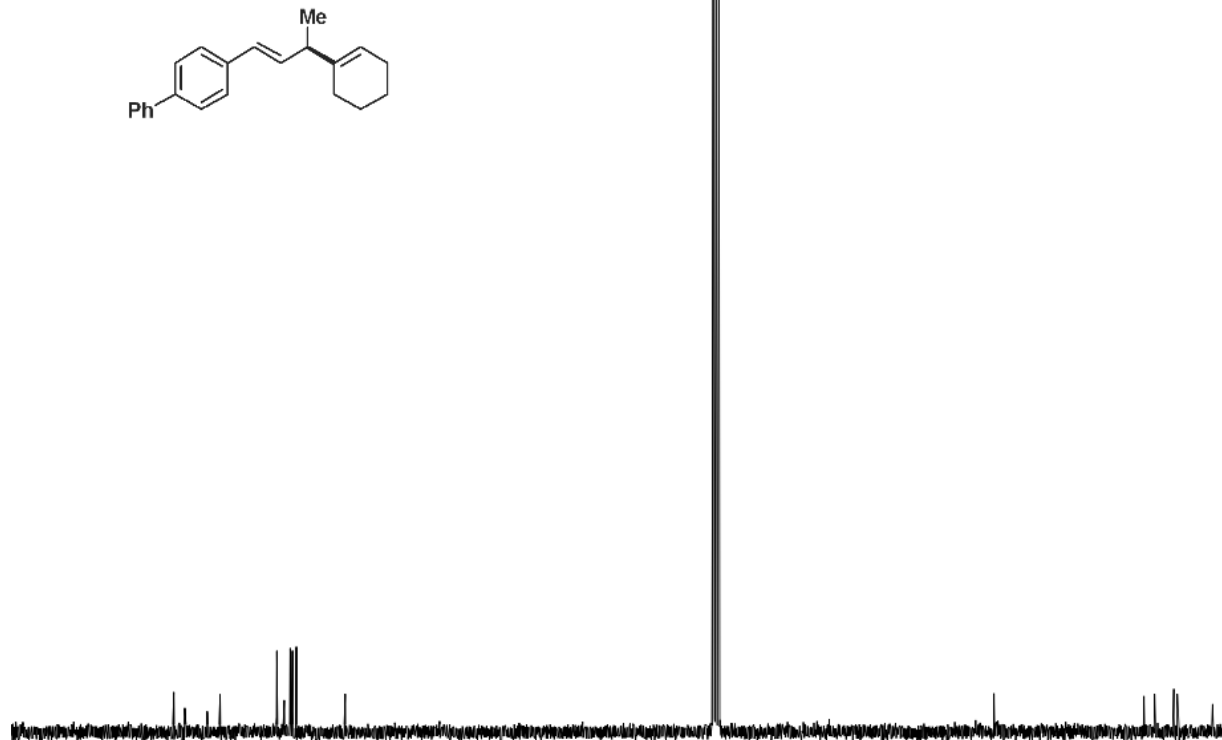

$80{ }^{1} 7$ 
Z-19; ${ }^{1} \mathrm{H}$ NMR (400MHz, $\left.\mathrm{CDCl}_{3}\right) ;{ }^{13} \mathrm{C}$ NMR (100 MHz, $\left.\mathrm{CDCl}_{3}\right)$

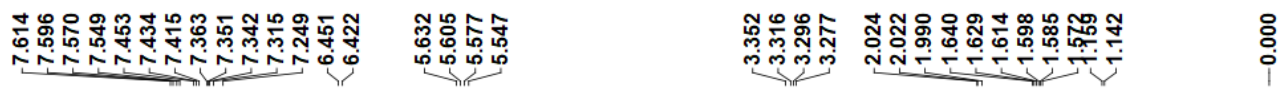<smiles>CC(/C=C\c1ccc(-c2ccccc2)cc1)C1=CCCCC1</smiles>
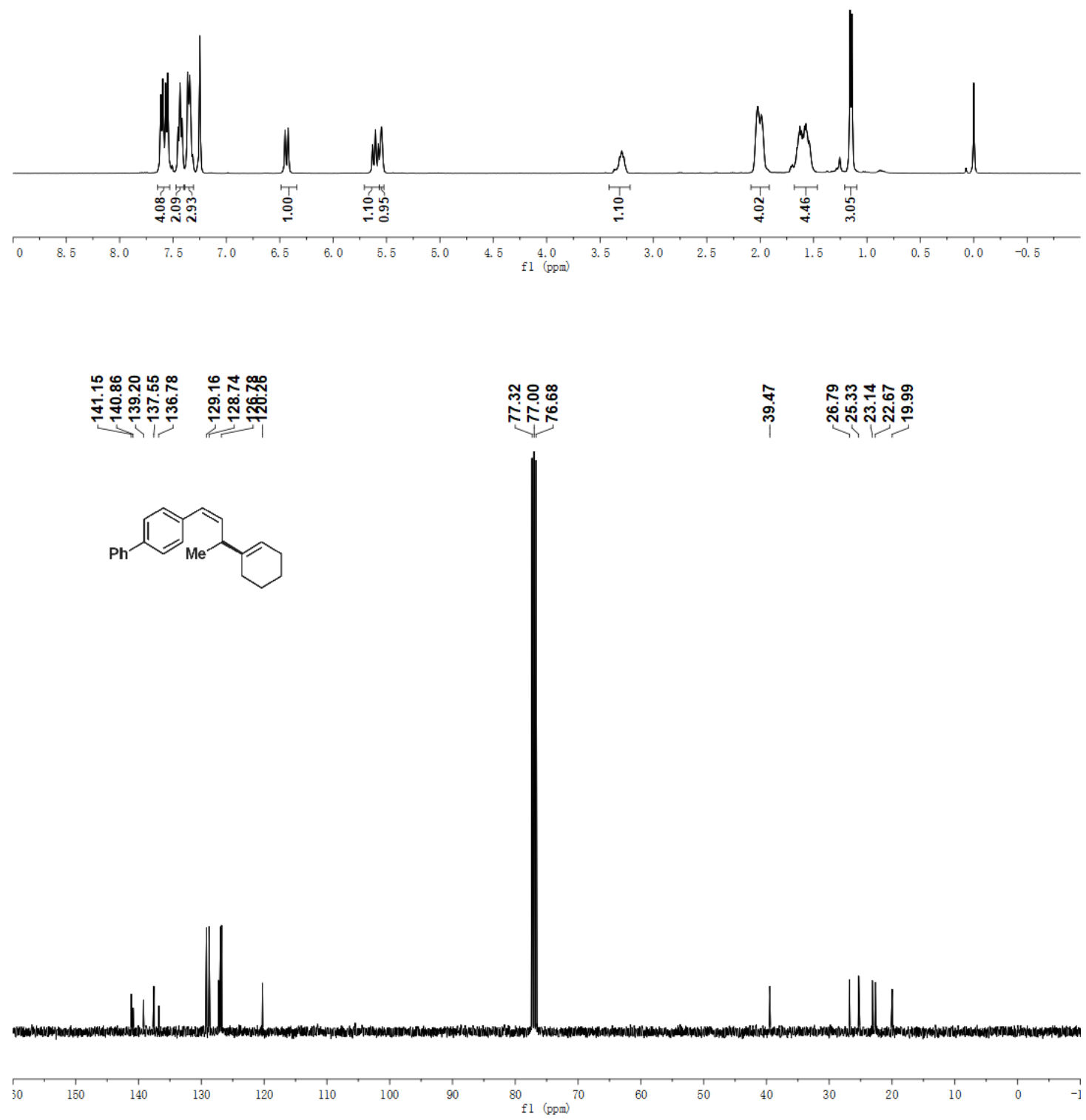

1. กูกสำ 
Z-3a; ${ }^{1} \mathrm{H}$ NMR (400MHz, $\left.\mathrm{CDCl}_{3}\right) ;{ }^{13} \mathrm{C}$ NMR (100 MHz, $\left.\mathrm{CDCl}_{3}\right)$

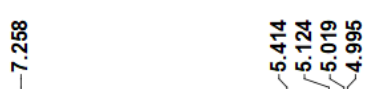

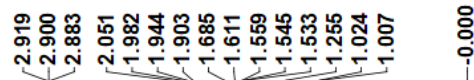
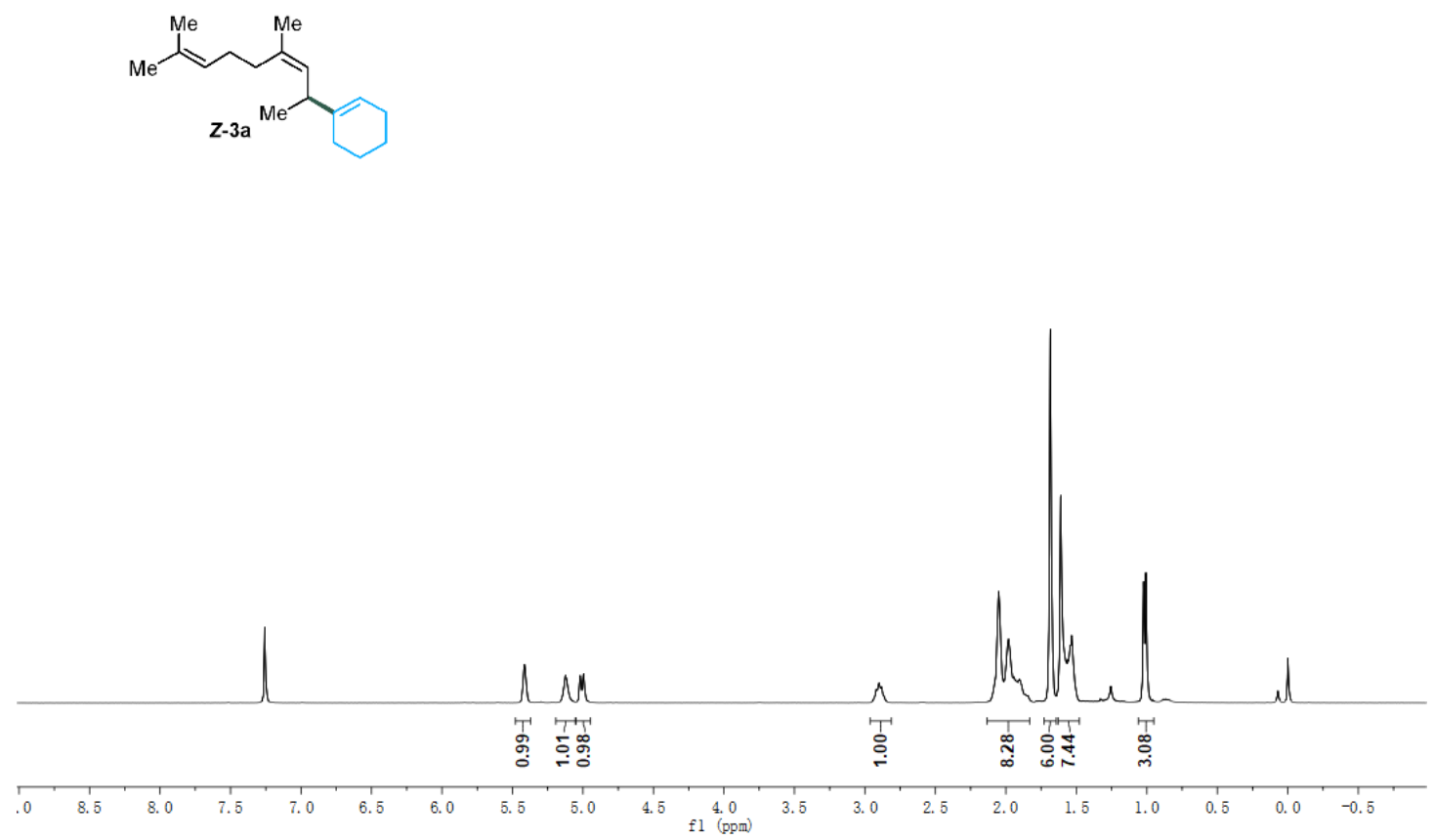

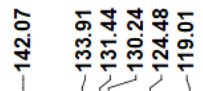
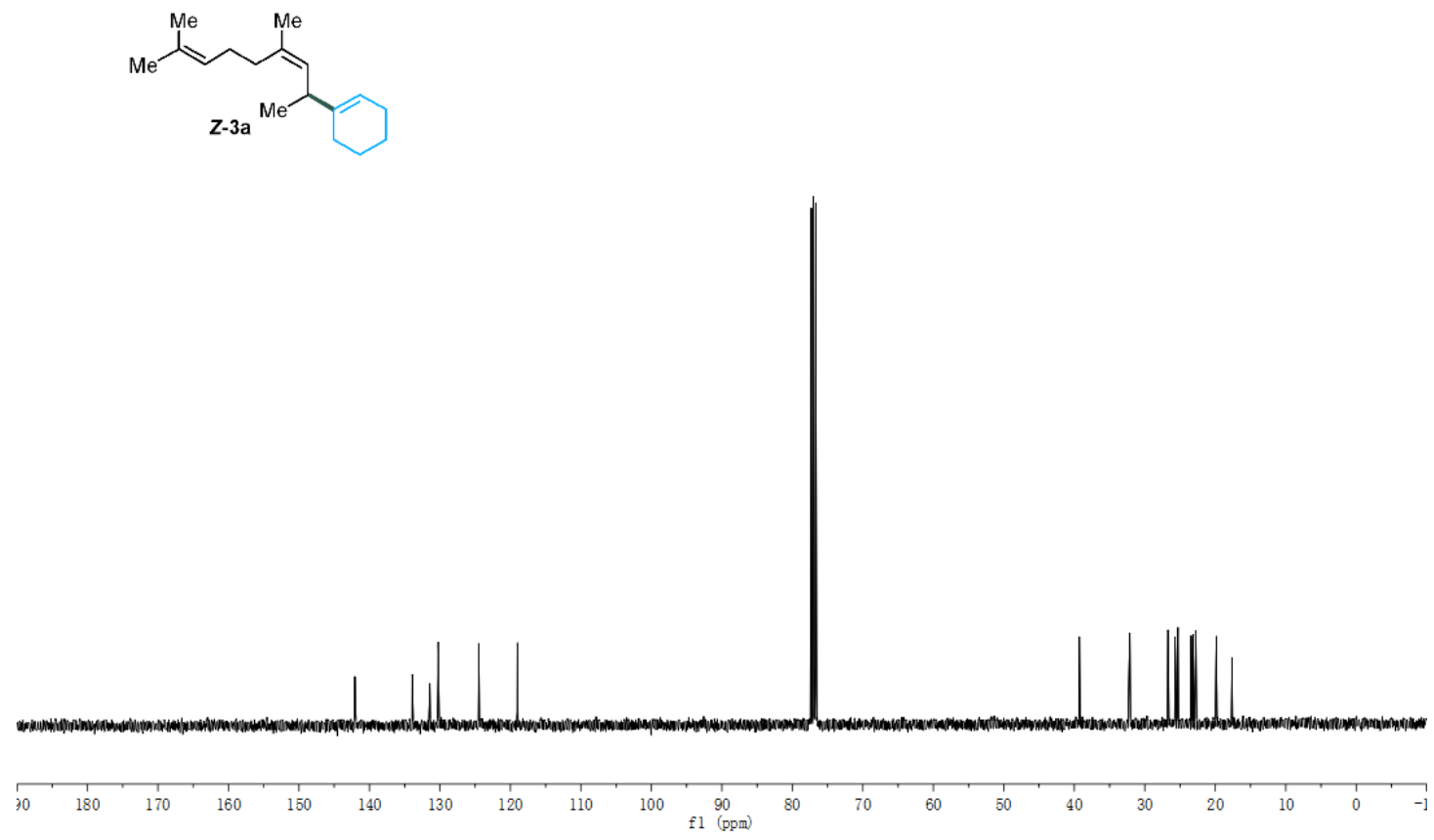
Complex A; ${ }^{1}$ H NMR (400MHz, THF-d8); ${ }^{31}$ P NMR (161 MHz, THF-d8)

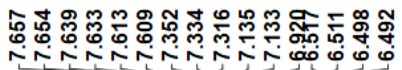

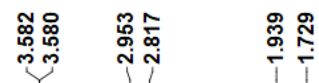

웅

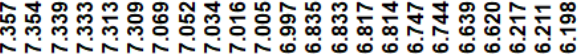
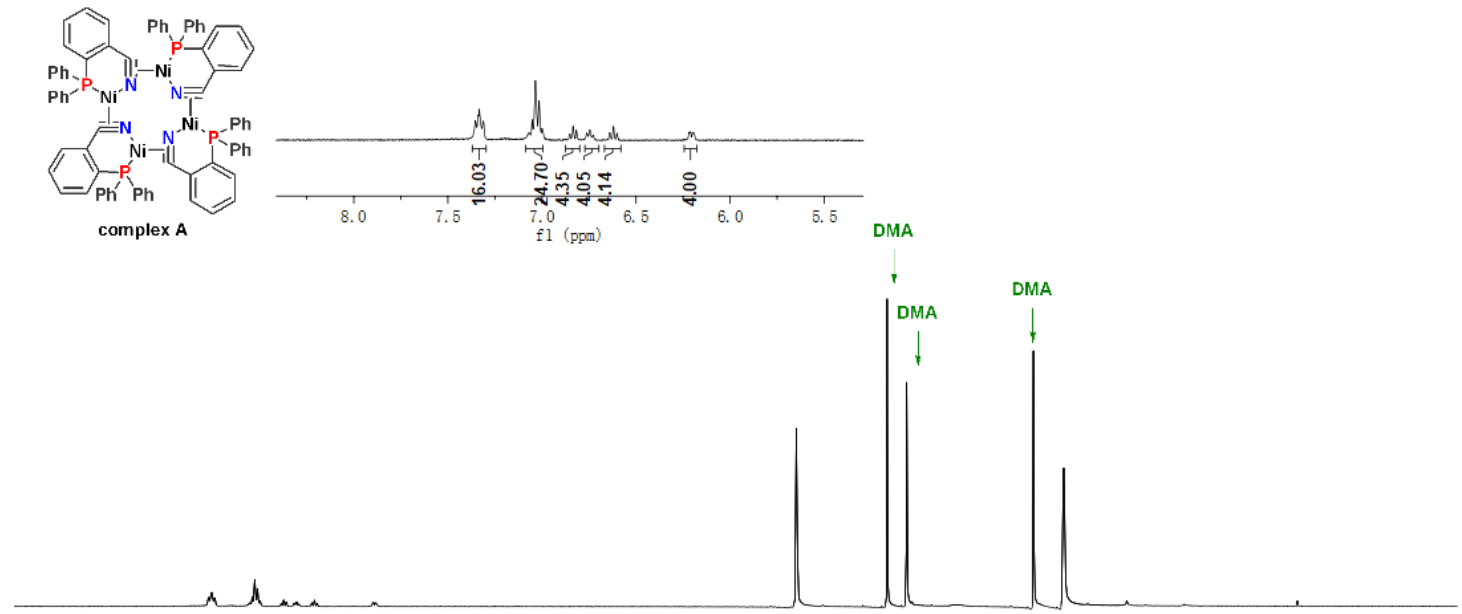

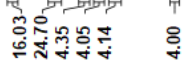

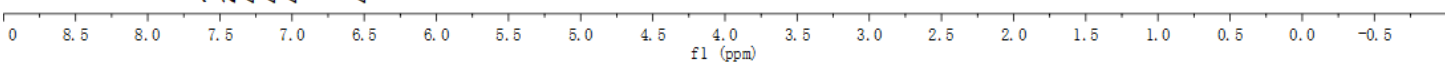

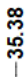
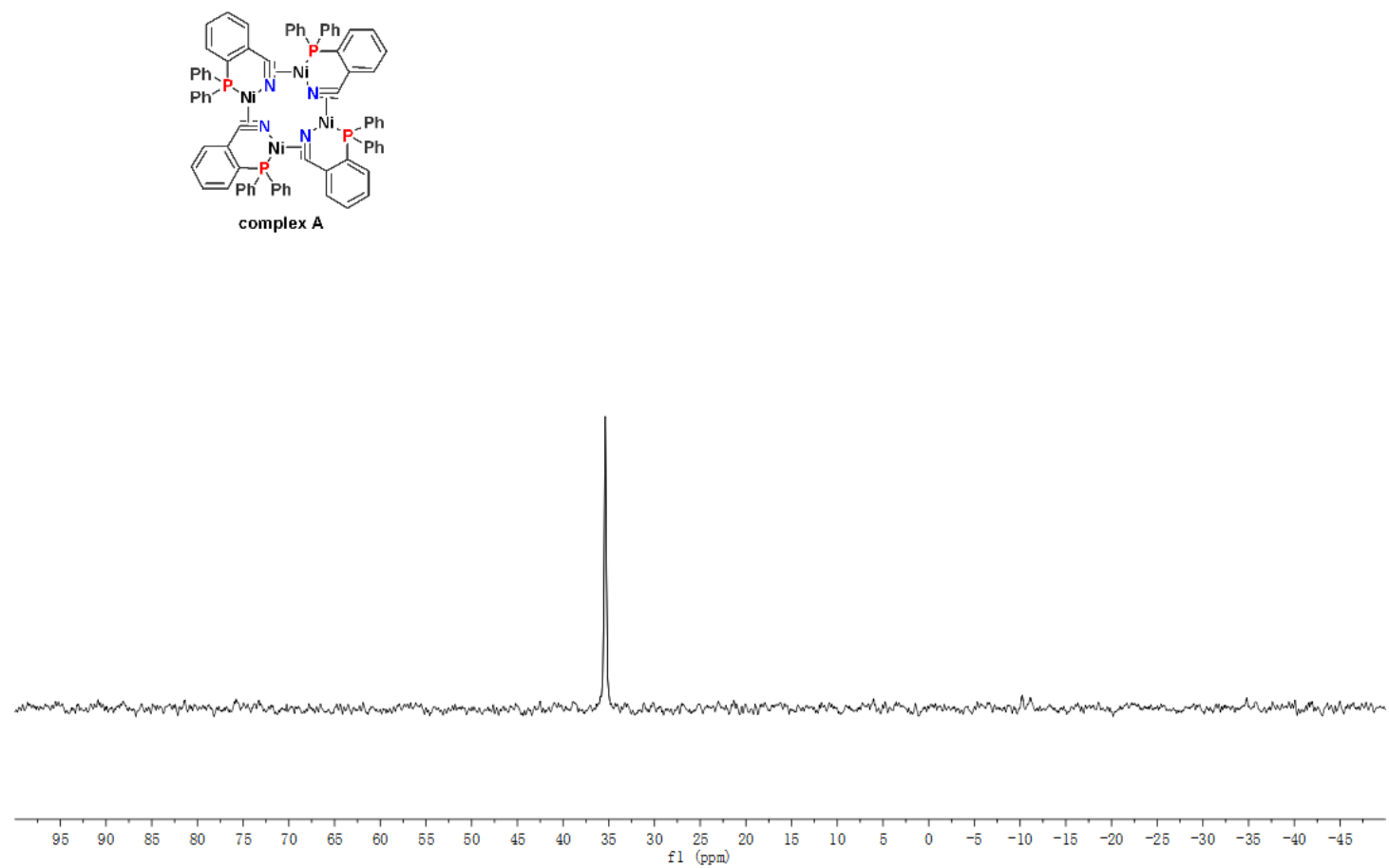